\title{
Resolving the Ultrastructural Organization of \\ Synaptic Vesicle Pools at Hippocampal Mossy Fiber and Schaffer Collateral Synapses
}

\author{
Dissertation \\ for the award of the degree \\ "Doctor rerum naturalium" (Dr. rer. nat.) \\ of the Georg-August-Universität Göttingen
}

within the doctoral program Biology and Psychology

of the Georg-August University School of Science (GAUSS)

Submitted by

\section{Lydia Susann Bickford Maus}

from Cambridge, Massachusetts, United States of America

Göttingen, 2020 


\section{Members of the Thesis Advisory Committee:}

\section{Prof. Dr. Nils Brose}

Department of Molecular Neurobiology, Max Planck Institute for Experimental Medicine, Göttingen

\section{Prof. Dr. Tobias Moser}

Molecular anatomy, physiology, and pathology of sound coding and prosthetics, Institute for Auditory Neuroscience \& InnerEarLab, University Medical Center Göttingen, Göttingen

Prof. Dr. Silvio O. Rizzoli

Department of Neuro- and Sensory Physiology, University Medical Center Göttingen, Göttingen

\section{Members of the Examination Board}

Prof. Dr. Nils Brose (1 $1^{\text {st }}$ Reviewer)

Department of Molecular Neurobiology, Max Planck Institute for Experimental Medicine, Göttingen

Prof. Dr. Tobias Moser (2 ${ }^{\text {nd }}$ Reviewer)

Molecular anatomy, physiology, and pathology of sound coding and prosthetics, Institute for Auditory Neuroscience \& InnerEarLab, University Medical Center Göttingen, Göttingen

\section{Further members of the Examination Board}

\section{Prof. Dr. Silvio O. Rizzoli}

Department of Neuro- and Sensory Physiology, University Medical Center Göttingen, Göttingen

\section{Prof. Dr. André Fiala}

Department of molecular neurobiology of behavior, University of Göttingen, Göttingen

\section{Prof. Dr. Carolin Wichmann}

Institute for Auditory Neuroscience and InnerEarLab, University Medical Center, Göttingen

\section{Prof. Dr. Stefan Jakobs}

Department of NanoBiophotonik, Max Planck Institute for Biophysical Chemistry, Göttingen

Date of the oral examination: September 14, 2020 


\section{Declaration}

I hereby declare that this thesis was written independently and with no other sources and aids than quoted.

Lydia Susann Bickford Maus

Göttingen, $10^{\text {th }}$ of August, 2020 


\section{Table of Contents}

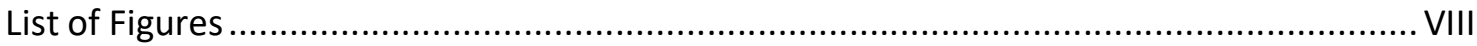

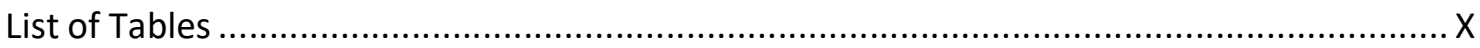

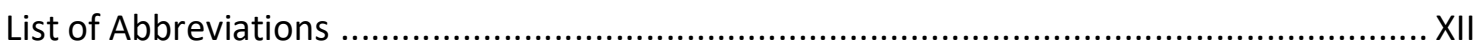

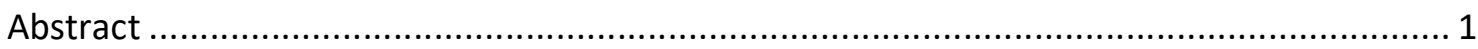

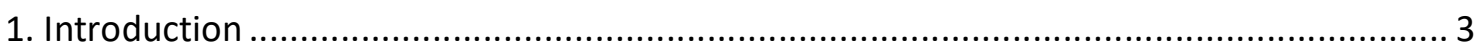

1.1. Synaptic ultrastructure and function: a historical perspective ................................ 5

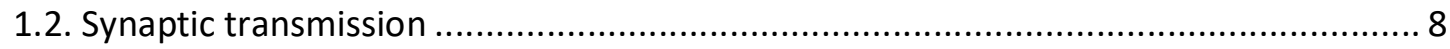

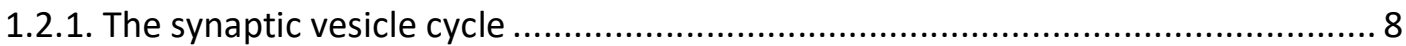

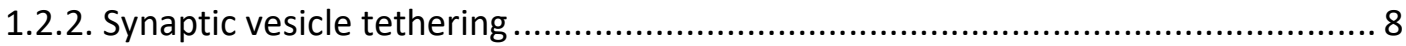

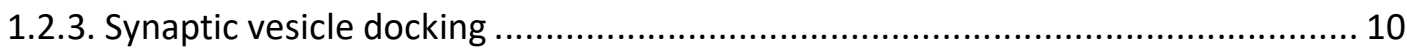

1.2.4. The readily-releasable pool of synaptic vesicles............................................. 10

1.2.5. Synaptic vesicle priming and exocytosis.................................................... 11

1.2.6. Molecularly primed synaptic vesicles are morphologically docked .................... 12

1.2.7. Release probability and short-term plasticity ............................................. 13

1.3. The role of functional heterogeneity in brain circuits: the hippocampus .................. 18

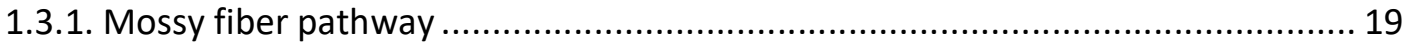

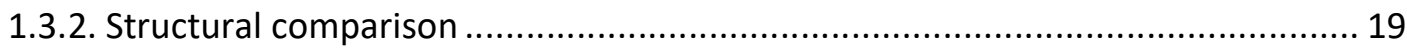

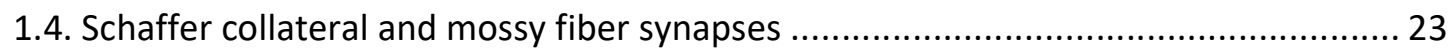

1.4.1. Functional differences between synapses .................................................... 23

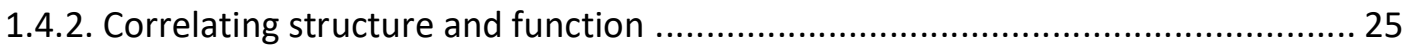

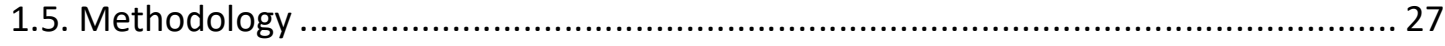

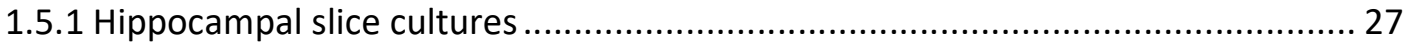

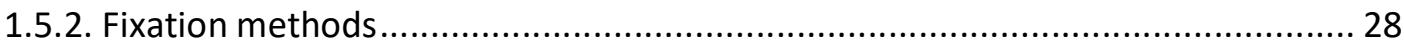

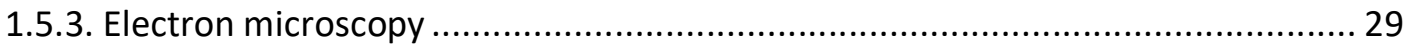

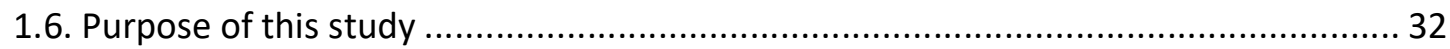

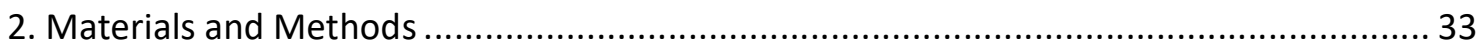

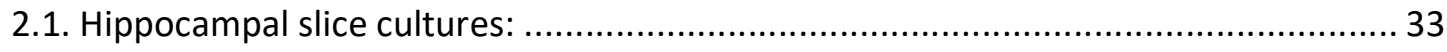

2.1.1. Slice cultures made from C57BL/6N wild-type mice...................................... 33

2.1.2. Slice cultures made from Munc13-deficient and control mice ........................... 34

2.2. High-pressure freezing, automated freeze substitution, and sample preparation for

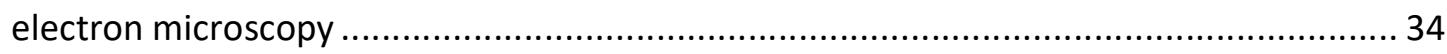

2.2.1. High-pressure freezing of organotypic slice cultures................................... 34 


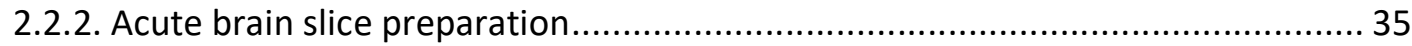

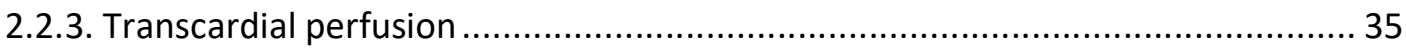

2.2.4. Immersion fixation of hippocampal slice cultures .......................................... 36

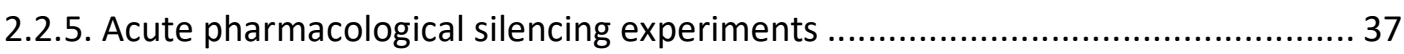

2.2.6. Pharmacological manipulation of presynaptic cAMP ....................................... 38

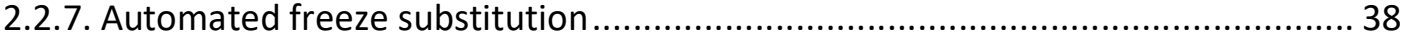

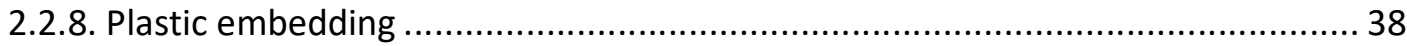

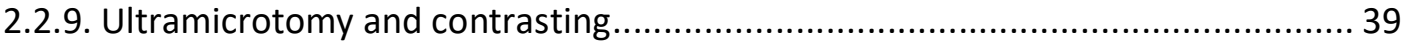

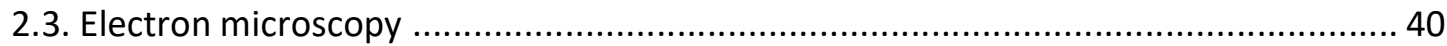

2.3.1. Transmission electron microscopy imaging and analysis................................. 40

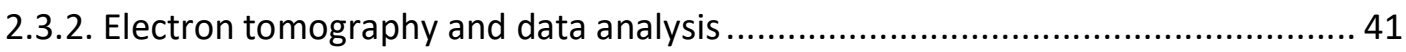

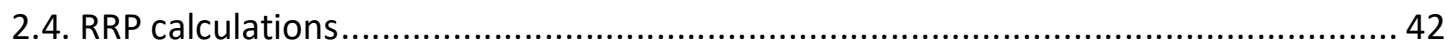

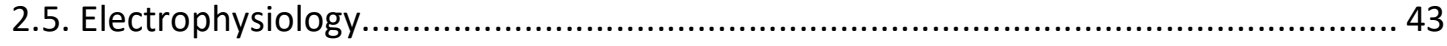

2.5.1. Miniature excitatory postsynaptic currents in CA3 pyramidal neurons in slice culture at DIV14

2.5.2. Release probability and short-term plasticity of Schaffer collateral and mossy fiber synapses in slice cultures at DIV14 and DIV28 ............................................. 45

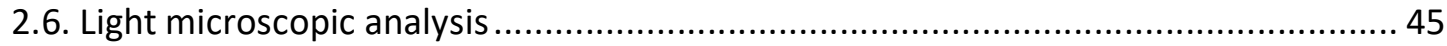

2.6.1. Sample preparation for confocal microscopy ........................................... 45

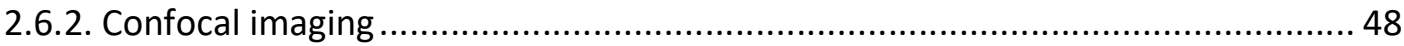

2.6.3. Stimulated emission depletion microscopy.................................................... 49

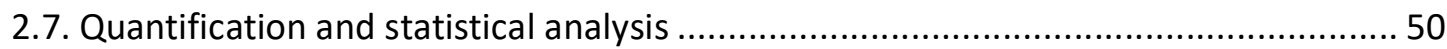

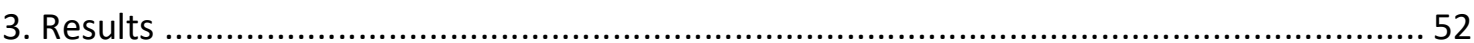

3.1. Differences in Schaffer collateral and mossy fiber synapse release probability are paralleled by synapse-specific differences in synaptic vesicle organization..................... 52

3.1.1. Intact target specificity of the mossy fiber pathway in hippocampal organotypic slice cultures

3.1.2. Comparative functional analysis of Schaffer collateral and mossy fiber synapses in hippocampal slice cultures

3.1.3. Comparative ultrastructural analysis of Schaffer collateral and mossy fiber synapses in hippocampal slice cultures

3.1.4. Synapse-specific differences in vesicle organization characterize Schaffer collateral and mossy fiber synapses in high-pressure frozen acute hippocampal slice preparations

3.1.5. Perfusion fixation of brain tissue causes a severe reduction in docked and membrane-proximal synaptic vesicles in mossy fiber-CA3 synapses. 
3.1.6. Munc13 priming molecules are essential for vesicle docking in hippocampal mossy fiber synapses

3.1.7. Acute pharmacological inhibition of action potential firing does not alter docked synaptic vesicle density in mossy fiber synapses

3.1.8. Pharmacological manipulation of presynaptic cAMP only minimally impacts synaptic vesicle organization in mossy fiber synapses ................................................ 71

3.2. Morphological heterogeneity of the docked vesicle pool in mossy fiber synapses .... 73

3.2.1. Large, clear-core vesicles dock exclusively at mossy fiber active zones in hippocampal slice cultures

3.2.2. Mossy fiber giant vesicles are not a consequence of the slice culture procedure 74

3.2.3. The organization of giant vesicles at mossy fiber active zones is sensitive to aldehyde fixation

3.2.4. Acute pharmacological blockade of network activity does not alter giant vesicle organization .....

3.2.5. Giant vesicles are present, but do not dock in Munc13-deficient mossy fiber synapses

3.2.6. Mossy fiber giant vesicles are the morphological correlate of giant mEPSCs recorded in CA3 pyramidal neurons...

3.2.7. Pharmacological manipulation of presynaptic CAMP does not alter giant vesicle organization

3.2.8. Giant vesicles may originate in granule cell somas and are not restricted to immature mossy fiber synapses....

3.3. DCVs dock at the active zone in mossy fiber synapses.

3.3.1. DCVs dock at mossy fiber active zones in slice cultures and acute slice preparations

3.3.2. Munc13 priming proteins are essential for DCV docking at mossy fiber synapses and lead to accumulation of DCVs in proximity to the active zone

3.3.3. Acute pharmacological silencing of mossy fiber synapses does not change the accumulation and distribution of DCVs

3.3.4. Pharmacological manipulation of presynaptic cAMP affects DCV distribution and

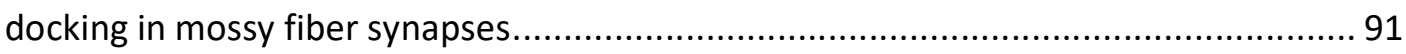

3.4. Morphological RRP estimates from past and present studies................................. 93

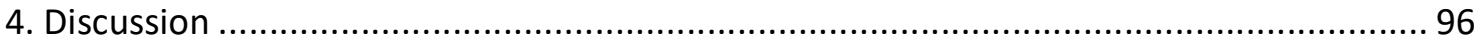

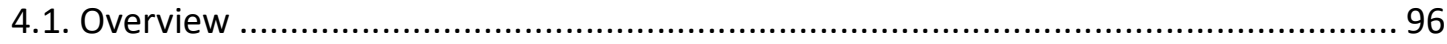

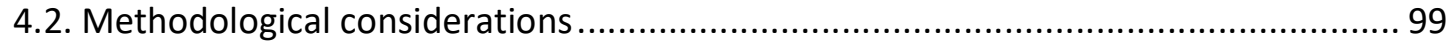

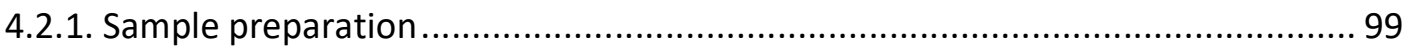


4.2.2. Limitations of $2 \mathrm{D}$ electron microscopy and advantages of $3 \mathrm{D}$ electron tomography

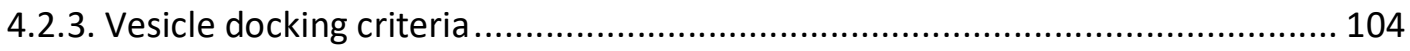

4.3. The RRP and morphologically docked vesicles.................................................. 107

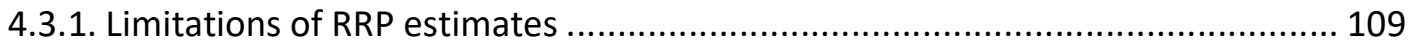

4.4. Morphological correlates of mossy fiber facilitation ........................................... 111

4.5. Synaptic vesicles, giant vesicles, and dense core vesicles at mossy fiber active zones

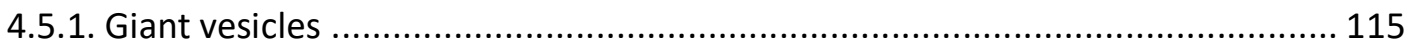

4.5.2. Pharmacological enhancement of release probability ................................... 118

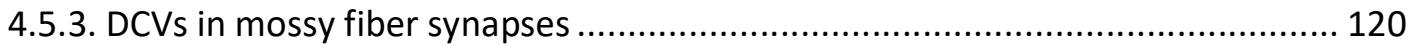

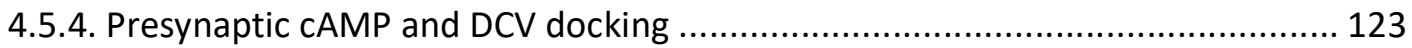

4.6. Development of Schaffer collateral and mossy fiber synapses in slice culture ......... 125

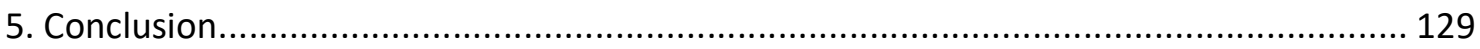

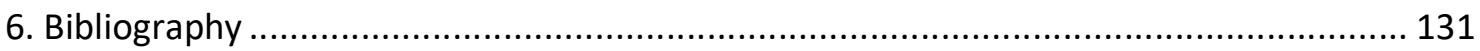

7. Supplemental Figures and Tables .................................................................... XV

7.1. Perfusion fixation changes the localization of DCVs in mossy fiber synapses.............XV

7.2. The effect of aldehydes on synaptic vesicle docking is depth-dependent in immersion-

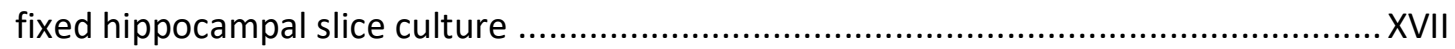

7.3. Presynaptic structural parameters are essentially unaffected by acute pharmacological manipulation of CAMP in hippocampal mossy fiber boutons................XX

7.4. Mitochondria in Schaffer collateral and mossy fiber synapses .............................XXVI

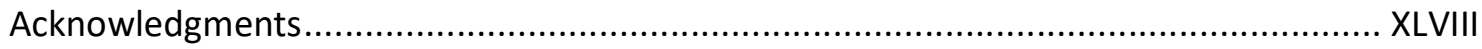

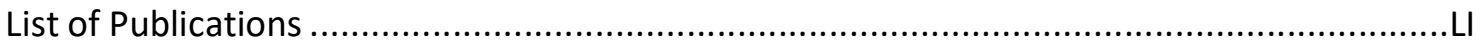




\section{List of Figures}

Figure 1. Synaptic ultrastructure.

Figure 2 . The synaptic vesicle cycle and molecular regulation of stimulus-evoked secretion at presynaptic active zones.

Figure 3. Hippocampal network connectivity and the mossy fiber pathway. ..................... 18

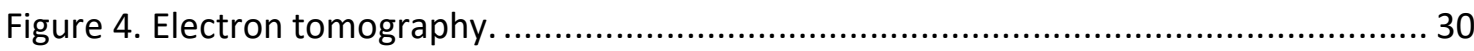

Figure 5. Light microscopic analysis of the mossy fiber-CA3 pathway in hippocampal organotypic slice cultures at DIV14 and 28.

Figure 6. Ultrastructural organization of mossy fiber-CA3 synapses in organotypic

hippocampal slices prepared by high-pressure freezing and freeze substitution. 55

Figure 7. Comparative ultrastructural analysis of mossy fiber-CA3 synapse morphology in organotypic and ex vivo hippocampal preparations. 56

Figure 8. Comparison of Schaffer collateral and mossy fiber release probability and shortterm plasticity characteristics from hippocampal slice cultures at DIV14 and 28. 57

Figure 9. Comparative ultrastructural analysis of Schaffer collateral and mossy fiber active zones in organotypic hippocampal slice slices at DIV14 and DIV28.

Figure 10. Ultrastructural analysis from ex vivo preparations of hippocampal tissue. 64

Figure 11. Ultrastructural analysis of mossy fiber active zones in Munc13-deficient and control slice cultures at DIV28.

Figure 12. Ultrastructural analysis of vesicle pools after acute pharmacological manipulations of mossy fiber synapses in organotypic slice cultures at DIV14 and DIV28 ... 70 Figure 13. Three morphologically distinct vesicles dock at hippocampal mossy fiber synapses.

Figure 14. Distribution of giant vesicles in mossy fiber synapses from slice cultures, acute slice preparations, and perfusion-fixed hippocampal tissue. 76

Figure 15. Distribution of giant vesicles in mossy fiber synapses from slice cultures after acute silencing, genetic silencing, or after pharmacological manipulation of release probability.

Figure 16. Electrophysiological and morphological analysis of giant vesicles in mossy fiber synapses.

Figure 17. Giant vesicles may have somatic origins and are not merely an early developmental phenomenon.

Figure 18. Spatial distribution of DCVs in mossy fiber synapses in slice cultures and acute slice preparations.

Figure 19. Spatial distribution of DCVs in mossy fiber synapses in genetically and pharmacologically manipulated slice cultures. 90 Figure 20. Spatial distribution of DCVs in mossy fiber-CA3 synapses in perfusion-fixed ex vivo preparations.

Figure 21. Ultrastructural analysis of docked synaptic vesicles at mossy fiber synapses from immersion-fixed slice cultures at DIV28. XVIII 
Figure 22. Ultrastructural analysis of mossy fiber boutons after forskolin treatment in slice

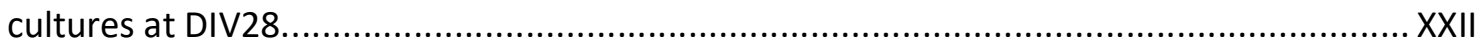

Figure 23. Distributions of vesicle diameter in Schaffer collateral and mossy fiber synapses from acute and cultured slice preparations. XXIII

Figure 24. Vesicle diameter distributions in mossy fiber-CA3 synapses after genetic or pharmacological manipulations of synaptic transmission..........................................XIV Figure 25. Vesicle diameter distributions in mossy fiber-CA3 synapses from aldehyde-fixed ex vivo preparations and high-pressure frozen organotypic slice cultures. XXV Figure 26. 2D ultrastructural comparison of Schaffer collateral and mossy fiber-CA3 synapses from DIV28 organotypic slice cultures....................................................................XXVI 


\section{List of Tables}

Table 1. Comparative morphologies of Schaffer collateral and mossy fiber synapses from ultrastructural studies.

Table 2. Functional properties of Schaffer collateral and mossy fiber synapses.

Table 3. Reagents and equipment for hippocampal organotypic slice cultures. 33

Table 4. Reagents and equipment for high-pressure freezing. 34

Table 5. Reagents and equipment for chemical fixation. 35

Table 6. Reagents used for pharmacological treatment of organotypic slices. 37

Table 7. Reagents and equipment for sample processing and preparation for electron microscopy. 40

Table 8. Imaging software and equipment for 2D electron microscopy and 3D electron tomography. 41

Table 9. Software used for electrophysiology acquisition and analysis. 43

Table 10. Reagents and equipment used for immunostaining and light microscopy. 46 Table 11. Estimating the total change in membrane capacitance of docked and membraneproximal vesicles in cultured mossy fiber synapses at DIV14.

Table 12. Estimating the total change in membrane capacitance of docked and membraneproximal vesicles in cultured mossy fiber synapses at DIV28. 94

Table 13. Published presynaptic capacitance studies of mossy fiber boutons. 94 Table 14. Statistical analysis of evoked EPSCs recorded in CA3 and CA1 pyramidal neurons in hippocampal slice cultures. XXVII Table 15. Statistical analysis of vesicle distributions in Schaffer collateral and mossy fiberCA3 synapses in organotypic slice cultures high-pressure frozen at DIV14. XXVIII Table 16. Statistical analysis of vesicle diameters in Schaffer collateral and mossy fiber-CA3 synapses in organotypic slice cultures high-pressure frozen at DIV14. XXIX Table 17. Statistical analysis of vesicle distributions in Schaffer collateral and mossy fiberCA3 synapses from organotypic slice cultures high-pressure frozen at DIV28. $X X X$ Table 18. Statistical analysis of vesicle diameters in Schaffer collateral and mossy fiber-CA3 synapses in organotypic slice cultures high-pressure frozen at DIV28. XXXI Table 19. Statistical analysis of vesicle distributions in Schaffer collateral and mossy fiberCA3 synapses in wild-type acute slice preparations high-pressure frozen at P18. XXXII Table 20. Statistical analysis of vesicle diameters in Schaffer collateral and mossy fiber-CA3 synapses from wild-type acute slice preparations high-pressure frozen at P18. XXXIII Table 21. Statistical analysis of vesicle distributions in mossy fiber synapses from wild-type mice perfusion-fixed at $\mathrm{P} 28$. XXXIV Table 22. Statistical analysis of vesicle diameters in mossy fiber synapses from wild-type mice perfusion-fixed at P28. $\mathrm{XXXV}$ Table 23. Statistical analysis of vesicle distributions in mossy fiber synapses from Munc13deficient and control slice cultures high-pressure frozen at DIV28. XXXVI Table 24. Statistical analysis of vesicle diameters in mossy fiber synapses from Munc13deficient and control slice cultures high-pressure frozen at DIV28. XXXVII Table 25. Statistical analysis of vesicle distributions in mossy fiber-CA3 synapses after acute pharmacological silencing in slice cultures high-pressure frozen at DIV14. XXXVIII 
Table 26. Statistical analysis of vesicle distributions in mossy fiber-CA3 synapses after acute pharmacological silencing in slice cultures high-pressure frozen at DIV14. XXXIX Table 27. Statistical analysis of vesicle distributions in mossy fiber synapses after pharmacological manipulation of presynaptic CAMP in slice cultures high-pressure frozen at DIV28. $\mathrm{XL}$

Table 28. Statistical analysis of vesicle diameters in mossy fiber synapses after pharmacological manipulation of presynaptic CAMP in slice cultures high-pressure frozen at DIV28. $\mathrm{XLI}$ Table 29. Statistical analysis of mEPSCs in CA3 pyramidal neurons recorded in slice culture at DIV14. XLII

Table 30. Statistical analysis of docked vesicle distributions and diameters in mossy fiber synapses 5-11 $\mu \mathrm{m}$ from the tissue surface in slice cultures immersion-fixed at DIV28. _ XLIII Table 31. Statistical analysis of docked vesicle distributions and diameters in mossy fiber synapses 20-22 $\mu \mathrm{m}$ from the tissue surface in slice cultures immersion-fixed at DIV28. _ XLIV Table 32. Statistical analysis of mossy fiber boutons imaged with STED microscopy in slice cultures after acute forskolin or control treatments at DIV28. XLV Table 33. Statistical analysis of 2D ultrastructural features in mossy fiber boutons after acute forskolin and control treatment in slice cultures high-pressure frozen at DIV28. XLVI Table 34. Statistical analysis of 2D presynaptic ultrastructural features of Schaffer collateral and mossy fiber synapses in slice cultures high-pressure frozen at DIV28 XLVII 


\section{List of Abbreviations}

\begin{tabular}{|c|c|}
\hline 2D & Two-dimensional \\
\hline 3D & Three-dimensional \\
\hline AC1 & Adenylate cyclase 1 \\
\hline ACSF & Artificial cerebrospinal fluid \\
\hline AFS & Automated freeze substitution \\
\hline AMPA & a-Amino-3-hydroxy-5-methyl-4-isoxazolepropionic acid \\
\hline ATP & Adenosine triphosphate \\
\hline BDNF & Brain-derived neurotrophic factor \\
\hline BSA & Bovine serum albumin \\
\hline CA & Cornu ammonis area \\
\hline CAMP & Cyclic adenosine monophosphate \\
\hline CAPS & Calcium-dependent secretion activator \\
\hline CTRL & Control \\
\hline D-AP5 & D-(-)-2-Amino-5-phosphonopentanoic acid \\
\hline DCG-IV & (2S,1'R,2'R,3'R)-2- (2,3-dicarboxycyclopropyl)glycine \\
\hline DCV & Dense-core vesicle \\
\hline Den.t & Dendrite \\
\hline DG & Dentate gyrus \\
\hline DIV & Days in vitro \\
\hline DKO & Double knock-out \\
\hline DMSO & Dimethyl sulfoxide \\
\hline E & Embryonic day \\
\hline EC & Entorhinal cortex \\
\hline EM & Electron microscopy \\
\hline Epac & Exchange protein directly activated by cAMP \\
\hline EPSCs & Excitatory postsynaptic currents \\
\hline FIB-SEM & Focused ion-beam scanning electron microscopy \\
\hline GA & Glutaraldehyde \\
\hline GABA & Y-Aminobutyric acid \\
\hline HPF & High-pressure freezing \\
\hline LS & Loosely docked \\
\hline LTP & Long-term potentiation \\
\hline mEPSC & Mini excitatory postsynaptic current \\
\hline mGluR2 & Metabotropic glutamate receptor type 2 \\
\hline MAP-2 & Microtubule-associated protein 2 \\
\hline Munc13 & Mammalian uncoordinated-13 \\
\hline NBQX & 2,3-Dioxo-6-nitro-1,2,3,4-tetrahydrobenzo[f]quinoxaline-7-sulfonamide \\
\hline NGS & Normal goat serum \\
\hline NMDAR & $\mathrm{N}$-methyl-D-aspartate receptor \\
\hline $\mathbf{P}$ & Postnatal day \\
\hline PB & Phosphate buffer \\
\hline PF & Perfusion fixative \\
\hline PFA & Paraformaldehyde \\
\hline
\end{tabular}




\begin{tabular}{l|l}
\hline PKA & Protein kinase $A$ \\
\hline PSD & Postsynaptic density \\
\hline RIM & Rab3-interacting molecule \\
\hline RBP & RIM binding protein \\
\hline RRP & Readily releasable pool \\
\hline SEM & Standard error of the mean \\
\hline SNARE & Soluble N-ethylmaleimide-factor attachment protein receptor \\
\hline STED & Stimulated emission depletion \\
\hline TEA & Tetraethylammonium \\
\hline TEM & Transmission electron microscope \\
\hline TS & Tightly docked \\
\hline TTX & Tetrodotoxin \\
\hline VC & Vehicle control \\
\hline VGCCs & Voltage-gated calcium channels \\
\hline
\end{tabular}




\section{Abstract}

The synapse is the functional unit of chemical communication between neurons in the brain. In order to relay information with spatiotemporal precision, the presynaptic compartment utilizes a specialized molecular machinery to organize synaptic vesicles at the presynaptic membrane so that upon the arrival of an action potential in the presynaptic terminal, synaptic vesicles can fuse with the plasma membrane and release their neurotransmitter contents into the synaptic cleft. Although many molecular components of this machinery are highly conserved, the functional transmitter release properties and plasticity characteristics can differ greatly between distinct neuron types, and even from synapse to synapse within the same cell. While critical for higher order cognitive processes, the underlying mechanisms of plastic changes in synaptic transmission remain poorly understood. Whether the fine ultrastructural organization of vesicles at presynaptic active zone release sites contributes to synaptic functional heterogeneity or to distinct plasticity states therefore remains an open question of considerable importance.

The aim of this study was to investigate whether the availability of docked and primed synaptic vesicles contributes to differences in release probability at two functionally wellcharacterized synapses, namely hippocampal Schaffer collateral and mossy fiber synapses. To address this question, I combined hippocampal slice culture, high-pressure freezing, automated freeze substitution, and electron tomography to accurately resolve the organization of vesicles at presynaptic active zones. Complementary electrophysiological analyses verified that hippocampal mossy fiber synapses exhibited a lower release probability and stronger short-term facilitation than Schaffer collateral synapses in our slice culture system. My ultrastructural analyses revealed that mossy fiber active zones harbored fewer docked synaptic vesicles and a prominent pool of putatively tethered synaptic vesicles. These data support the notion that the availability of docked and primed synaptic vesicles codetermines initial release probability at Schaffer collateral and mossy fiber synapses. I postulate that the abundance of membrane-proximal vesicles, ideally positioned to rapidly dock and prime at the plasma membrane during periods of increased synaptic activity, likely contributes to the facilitation characteristics of hippocampal mossy fiber synapses. Moreover, I hypothesize that the ratio of docked and tethered synaptic vesicles serves as a possible structural predictor of synaptic short-term plasticity characteristics. 
I discovered that three morphologically distinct types of vesicles docked at mossy fiber active zones: synaptic vesicles, giant vesicles (clear-core vesicles with a diameter exceeding $60 \mathrm{~nm}$ ), and dense-core vesicles (DCVs). All vesicle types required Munc13 priming molecules to dock at mossy fiber active zones. My data indicate that giant vesicles likely contain neurotransmitters and contribute to glutamatergic signaling at the mossy fiber-cornu ammonis area 3 synapse. I performed a quantitative morphometric analysis of respective vesicle pools at mossy fiber active zones and compared my data with published functional estimates of the readily releasable pool to demonstrate considerable overlap between the total numbers of morphologically docked and functionally primed and fusion-competent synaptic vesicles.

Having systematically quantified the ultrastructural profiles of mossy fiber active zones in synapses at rest, I examined whether changes in synaptic release probability induced by acute pharmacological manipulations of presynaptic cyclic adenosine monophosphate (cAMP) would trigger corresponding changes in vesicle organization. Interestingly, my data demonstrate that DCV, but not synaptic vesicle, docking is particularly sensitive to changes in presynaptic cAMP. These findings support a view in which mechanisms mediating CAMPdependent potentiation of glutamatergic transmission operate downstream of synaptic vesicle docking, and highlight the potential modulatory role of DCV-mediated neuropeptide release in mossy fiber plasticity processes.

In conclusion, my work demonstrates that initial release probability is co-determined by the availability of docked and primed vesicles and that the structural organization of vesicles at active zone release sites can indeed provide significant insight into key presynaptic functional properties. Moreover, it emphasizes that systematic and stringent high-resolution ultrastructural analyses are useful to reveal novel insight into ultrastructure-function relationships in other synapse types in the brain. 


\section{Introduction}

The brain is composed of billions of neurons that form trillions of synaptic connections. The chemical synapse represents the basic anatomical and functional unit underlying information transfer in the brain. Complementary and multidisciplinary approaches are required to achieve a comprehensive understanding of how synapses work at the level of their structural architecture, their molecular organization, and how their behavior is dynamically regulated during the process of information transfer. In neuronal networks in the brain, plastic changes in synaptic transmission efficacy are hypothesized to underlie complex and precise processes such as sensory processing, motor control, memory, and cognition (Purves et al., 2004).

My doctoral work particularly focuses on the relationship between synaptic ultrastructure and function. Although many model synapses have been functionally and morphologically characterized, it remains unclear how the structural organization of a synapse at the level of individual active zone release sites contributes to its functional properties. Electron microscopy (EM) remains the gold standard for accurately resolving the subcellular organization of lipid-bound synaptic organelles (i.e. synaptic vesicles) and synaptic subcompartments (i.e. active zone release sites). Recent studies have indicated that synapse function is profoundly influenced by subtle differences in the structural organization of synapses operating in the nanoscale range (Imig et al., 2014; Siksou et al., 2009a). Additionally they have emphasized that the detection of these changes is critically dependent on tissue preparation protocols for ultrastructural analysis (i.e. cryo-fixation) and on the imaging techniques used to resolve synaptic ultrastructure (i.e. electron tomography) (Imig et al., 2014; Siksou et al., 2009a).

My thesis work takes into account these important methodological considerations in a comparative ultrastructural analysis of hippocampal Schaffer collateral and mossy fiber synapses. These experiments are designed to provide an unprecedented, high-resolution perspective of active zone organization at the mossy fiber synapse, one of the most functionally and morphologically enigmatic synapses in the brain. Corresponding analyses of Schaffer collateral synapses, which represent arguably the most extensively characterized 
synapse in the brain, are designed to provide a reliable morphological and functional frame of reference. 

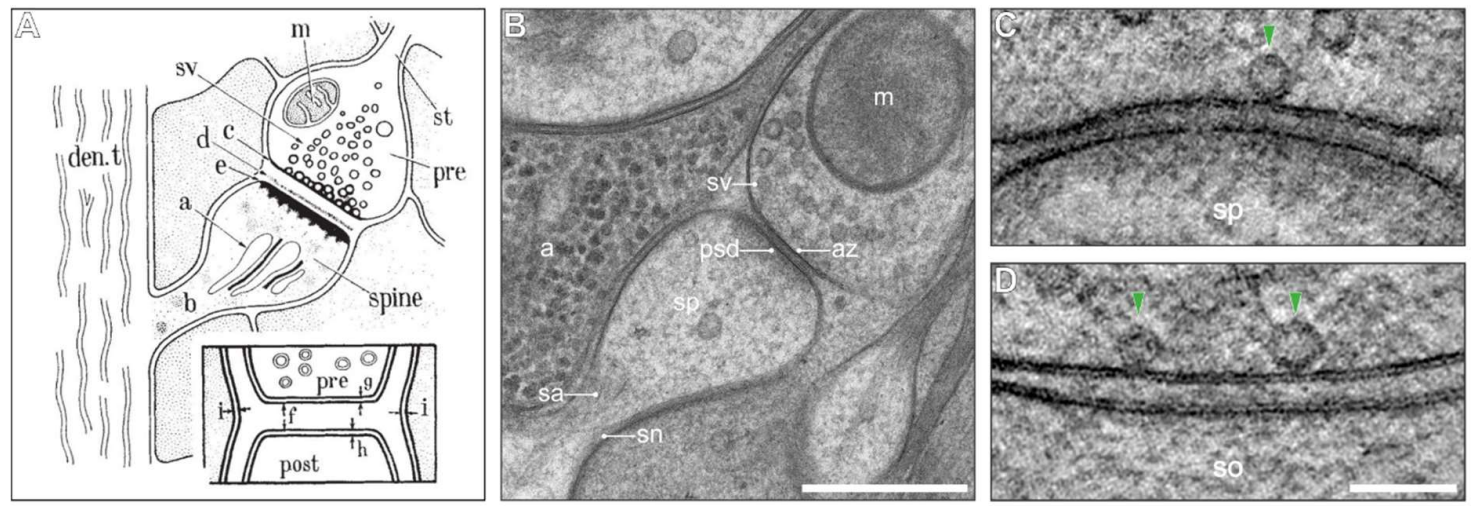

Figure 1. Synaptic ultrastructure.

(A) Schematic representing the first electron microscopic characterization of a small cortical spine synapse and its respective subcellular compartments. (B) Transmission electron micrograph of a small Schaffer collateral spine synapse in an organotypic hippocampal slice culture prepared by high-pressure freezing and freeze substitution (Imig and Cooper, 2017). (C and D) Characteristic features of asymmetrical (C; Grays Type I; excitatory) and symmetrical (D; Grays Type II; inhibitory) synapses. Docked vesicles are indicated with green arrowheads. Abbreviations: den.t, dendrite; sv, synaptic vesicle; sa, spine apparatus; sn, spine neck; sp, spine; so, soma; m, mitochondrion; az, active zone; a, astrocyte. Scale bars: $500 \mathrm{~nm}, \mathbf{B} ; 100 \mathrm{~nm}, \mathbf{C}$ and D. Permission \& Rights (A) from Gray, 1959 with permission from Copyright Clearance Center (license number 4786470690034); (B) from Imig and Cooper, 2017 with permission from Copyright Clearance Center (license number 4786471226892).

\subsection{Synaptic ultrastructure and function: a historical perspective}

Since its invention by Ernst Ruska in 1933 (Borries and Ruska, 1933; Ruska, 1933), the electron microscope has served as an invaluable research tool in the field of synaptic neurobiology. The structural composition of chemical synapses was first directly visualized in electron micrographs almost 65 years ago (Gray, 1959; Palay and Palade, 1955; De Robertis and Bennett, 1955). In his seminal study of synapses in the visual cortex of rats, Gray revealed a compartmental organization in which the axon of the signaling neuron terminated in a presynaptic bouton in close proximity to a postsynaptic compartment in the form of a dendritic spine (Figure 1 A and B) (Gray, 1959). Of critical importance to the understanding of synaptic function at the time, this ultrastructural view of the synapse revealed (i) that the axon terminal was filled with small vesicular organelles, termed synaptic vesicles, and (ii) that pre- and postsynaptic compartments were not in direct physical contact, but rather separated by what became known as the synaptic cleft. Subsequent studies performed in the frog neuromuscular junction built on this information by demonstrating that synaptic vesicles store and release chemical transmitter substances, fuse with the presynaptic membrane, and recycle to generate new vesicles during sustained activity (Ceccarelli et al., 1973; Heuser and 
Reese, 1973). Together, these findings provided the basis for understanding one of the most fundamental and pervasive ultrastructure-function relationships in neurobiology, namely that synaptic vesicles are the morphological correlates of the quantal neurotransmission identified by Castillo and Katz in 1954 (Castillo and Katz, 1954).

Importantly, EM enabled the visualization of sites of trans-synaptic information transfer at a subcellular scale and linked with the functional properties of individual synapse subtypes. Electron dense "membrane thickenings" indicative of functionally specialized cellular subcompartments were observed at opposing pre- and postsynaptic membranes across the synaptic cleft (Figure 1 A and B) (Gray, 1959; Palay, 1956). The active zone, a term reflecting observations that synaptic vesicles preferentially cluster and fuse at this presynaptic specialization (Couteaux and Pécot-Dechavassine, 1970), was postulated to provide the molecular and structural environment required for the spatio-temporally regulated release of neurotransmitter into the synaptic cleft (Phillips et al., 2001; Triller and Korn, 1985). The postsynaptic density, located in direct apposition to the active zone, was analogously postulated to provide the molecular and structural environment required to cluster membrane-bound receptors capable of receiving a transmitter signal (Okabe, 2007). The observation that the ultrastructural appearance of synaptic active zones and postsynaptic densities correlated with respect to the anatomical location, neurotransmitter content, and behavioral properties of the synapse ultimately led to a classification system relating the morphological and functional characteristics of synaptic subtypes (Colonnier, 1968; Eccles, 1964; Gray, 1959; Uchinozo, 1965). Grays Type I, or asymmetric, later classified as excitatory, form synapses onto dendritic shafts or spines, harbor spherical presynaptic vesicles, and have a pronounced postsynaptic density (Figure $1 \mathrm{C}$ ); Grays Type II, or symmetric, later classified as inhibitory, innervate neuronal soma and dendritic shafts, harbor pleiomorphic presynaptic vesicles, and have comparably sized active zones and postsynaptic densities (Figure $1 \mathrm{D}$ ) (Colonnier, 1968; Eccles, 1964; Gray, 1959; Uchinozo, 1965). Further support for this classification system was subsequently provided by the demonstration that synaptic vesicles in Type I and Type II synapses were immunoreactive against the main excitatory neurotransmitter glutamate and the main inhibitory neurotransmitter $\gamma$-aminobutyric acid (GABA), respectively (Barbaresi et al., 2001; Beaulieu and Somogyi, 1990). 

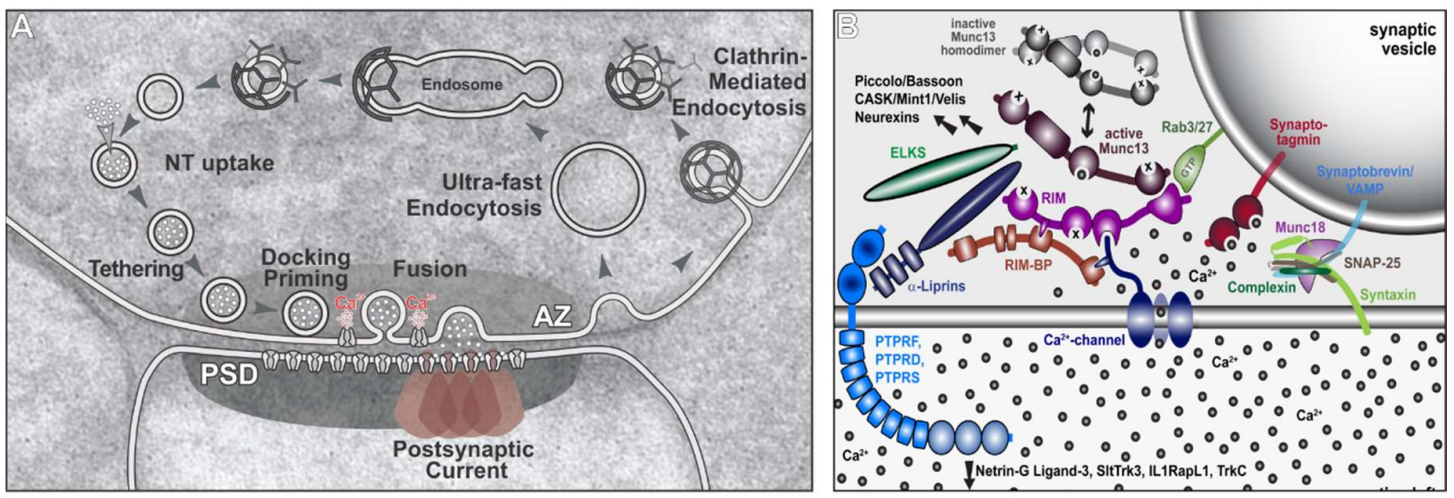

Figure 2. The synaptic vesicle cycle and molecular regulation of stimulus-evoked secretion at presynaptic active zones.

(A) Schematic of classical synaptic vesicle cycle. Newly synthesized neurotransmitters such as glutamate are actively transported from the cytoplasm into membrane-bound synaptic vesicles. Synaptic vesicles with transmitter cargo translocate to the plasma membrane where they dock and are molecularly primed for fusion with the plasma membrane upon calcium influx. Excess membrane caused by full collapse fusion with the plasma membrane is then recycled into the presynaptic terminal via both clathrin-dependent and clathrin-independent mechanisms. The recycled membrane is brought to endosomal structures where new synaptic vesicles are formed in a clathrin-dependent mechanism and then recycle back into the pool of synaptic vesicles. (B) Schematic of key regulatory molecules in excitation-secretion coupling at presynaptic active zones. These include: (i) active zone proteins such as ELKS, RIM-BP, and RIM that localize calcium channels to the presynaptic membrane and in proximity to synaptic vesicles, (ii) priming molecules such as Munc13s and Munc18 allow for synaptic vesicles to dock and prime at the plasma membrane bringing the vesicular and plasma membrane SNAREs close enough to interact, and (iii) SNARE complex components synaptobrevin, SNAP-25, and syntaxin, that catalyze the fusion of vesicular and plasma membranes. Abbreviations: NT, neurotransmitter; PSD, postsynaptic density; AZ, active zone. Permission \& Rights (B) from Südhof, 2012 with permission from Copyright Clearance Center (license number 4862960297628).

The aforementioned studies demonstrated the major contribution EM has made towards our understanding of the fundamental principles underlying synaptic transmission, and emphasize that systematic morphological analyses provide functional insight, even on a single synapse level. Although excitatory and inhibitory synapses represent the majority of synapses in the central nervous system, a considerably broader spectrum of synapse classes, neurotransmitters, and receptor subtypes is ultimately required to support complex brain functions. Moreover, the observation that synapses of a given class (i.e. Grays Type I or II) or neurotransmitter subtype can differ substantially in their efficacy of evoked transmitter release (Purves et al., 2004) emphasizes the need for further investigation of ultrastructurefunction relationships using refined methods and protocols designed to probe synaptic ultrastructure with higher stringency and to link electron microscopic observations with the molecular mechanisms underpinning fundamental synaptic properties. 


\subsection{Synaptic transmission}

\subsubsection{The synaptic vesicle cycle}

Despite the broad spectrum of synaptic neurotransmitters, morphologies, and behaviors required to support complex brain functions, all chemical synapses operate by a stereotypic principle. The propagation of information from one neuron to the next requires both an electrical and a chemical component. An action potential occurs when a change in the electrochemical gradient across the semi-permeable plasma membrane of a neuron causes the opening of voltage-gated ion channels. This signal propagates rapidly along the axon of a neuron until it reaches a presynaptic terminal. Within the presynaptic terminal, voltage-gated calcium channels (VGCCs) open and the influx of calcium into the presynaptic terminal triggers the fusion of synaptic vesicles with the active zone membrane and the subsequent release of their lumenal cargo, chemical messengers, or neurotransmitters, into the synaptic cleft in a process termed exocytosis (Figure 2). Once the neurotransmitter binds to a postsynaptic ionotropic receptor, the cycle of signal propagation continues as evoked currents are integrated in the postsynaptic neuron.

Stimulus-coupled exocytosis is tightly regulated by a complex molecular machinery that operates at multiple steps preceding synaptic vesicle fusion to regulate the spatio-temporal precision of transmitter release (Figure 2 B). Experimental approaches combining mouse genetics with functional assays and corresponding ultrastructural analyses have identified key presynaptic protein components of this machinery and generated a view of the synaptic vesicle cycle (Figure $2 \mathrm{~A}$ ) in which important regulatory steps can be related to the spatial organization of synaptic vesicles within the presynaptic terminal. This view illustrates a sequence of steps in which synaptic vesicles are: (i) filled with neurotransmitter, (ii) recruited to the active zone and loosely attached, or tethered, in proximity to the presynaptic membrane, (iii) docked in physical contact with the plasma membrane and rendered fusioncompetent in a molecular priming step, (iv) and fused with the plasma membrane upon detection of elevated presynaptic calcium (Figure $2 \mathrm{~A}$ ).

\subsubsection{Synaptic vesicle tethering}

The presynaptic compartment is ultrastructurally characterized by the accumulation synaptic vesicles at active zone release sites. Large, multivalent active zone scaffold molecules, 
including rab3-interacting molecule (RIM), bassoon, piccolo, and RIM binding protein (RBP) (Figure 2 B), create the local proteinaceous environment required to support stimulus-evoked neurotransmitter secretion. This protein network serves as a hub by mediating interactions between vesicular proteins (Geppert et al., 1997), VGCCs (Han et al., 2011; Südhof, 2012), and soluble components of the vesicle fusion apparatus (Figure 2) (Augustin et al., 1999; Brockmann et al., 2019; Südhof and Rizo, 2011). Although targeted genetic perturbations have implicated several components of the active zone scaffold in the recruitment of synaptic vesicles to active zone release sites (Han et al., 2011; Mukherjee et al., 2010; Südhof, 2012; Wang et al., 2016), a structural view of how this recruitment occurs is still evolving.

Experiments combining freeze-fracture with shallow etching provided early structural support for the concept that filamentous proteins play a role in the organization of synaptic vesicles at presynaptic active zones (Landis et al., 1988). Although rarely captured in aldehyde-fixed preparations, presynaptic filaments have since been described in a variety of species and synapse types prepared by rapid cryofixation, freeze substitution and plastic embedding (Bruckner et al., 2017; Cole et al., 2016; Siksou et al., 2009a; Stigloher et al., 2011; Vogl et al., 2015). More recently, cryo-electron tomographic reconstructions of frozenhydrated synaptosomes have enabled detection and quantification of filaments both between vesicles ("connectors"), and between vesicles and the presynaptic plasma membrane ("tethers") (Fernández-Busnadiego et al., 2010, 2013). The observation that the number and length of tethers per vesicle is inversely related to active zone proximity supported a model in which vesicles are initially tethered by single, long filaments ( $>5 \mathrm{~nm}$ ) before being anchored closer to the membrane by multiple short $(<5 \mathrm{~nm})$ tethers (FernándezBusnadiego et al., 2010, 2013). Moreover, an analysis of RIM1 $\alpha$ knock-out (KO) synapses revealed a perturbation of the organization of filaments and vesicles that implicated the active zone scaffold in these processes (Fernández-Busnadiego et al., 2013). However, the potential involvement of other active zone proteins in this phenotype must be considered, particularly in light of previous studies indicating that RIM is required for the correct active zone targeting of mammalian uncoordinated protein (Munc13) priming proteins (AndrewsZwilling et al., 2006). The detection of filamentous connectors and tethers in other cellular subcompartments is also consistent with the notion that multiple protein species, or protein isoforms, contribute to the formation of vesicle-associated filaments (Hallermann and Silver, 2013; Schrod et al., 2018). Although their molecular identity remains to be determined, 
tethers provide a structural framework and level of organization that is likely critical for maintaining the supply of vesicles to the active zone during sustained activity (Hallermann and Silver, 2013).

\subsubsection{Synaptic vesicle docking}

In contrast to tethering processes, the molecular mechanisms responsible for docking synaptic vesicles in close contact with the presynaptic membrane are relatively well understood (Südhof and Rizo, 2011). To appreciate the functional significance of the synaptic vesicle docking process, an understanding of multiple overlapping concepts is necessary. Accordingly, in this section I will discuss the concept of a readily-releasable pool (RRP) of synaptic vesicles in context with the molecular machinery required to generate it and ultimately trigger its fusion with the active zone membrane during stimulus-evoked neurotransmitter release.

\subsubsection{The readily-releasable pool of synaptic vesicles}

In order to fuse in response to the arrival of an action potential, synaptic vesicles must undergo a molecular priming process that renders them fusion competent. It is this priming process that ensures a RRP of vesicles is available to fuse and release neurotransmitters in response to the arrival of an action potential. The RRP of a synapse is typically assessed by functional means and corresponds to the number of vesicles that fuse with the synapse in response to strong, vesicle-depleting stimuli (Kaeser and Regehr, 2017; Neher, 2015). A range of functional assays has been developed to accommodate the specific demands of different experimental preparations (Ariel and Ryan, 2010; Bekkers and Stevens, 1991; Neher and Marty, 1982; Rizzoli and Betz, 2005; Rosenmund and Stevens, 1996; Schikorski and Stevens, 2001; Schneggenburger et al., 1999). These and associated caveats will be discussed in more detail in the methodological considerations in the discussion (see section 4.3.1 Limitations of RRP estimates). Major methodological differences include the type of stimulus applied to trigger transmitter release and the experimental means of detecting it. In low-density cultures, the rapid application of hypertonic sucrose reliably triggers fusion of the RRP (Bekkers and Stevens, 1991; Rosenmund and Stevens, 1996). However, this approach is not applicable to acute slice preparations where high frequency trains of action potentials are preferentially used to deplete the RRP (Schneggenburger et al., 1999; Thanawala and Regehr, 2013). Both approaches rely on a simultaneous measurement of postsynaptic responses to 
measure evoked transmitter release, which in glutamatergic neurons is manifest as excitatory postsynaptic currents (EPSCS). There are several caveats which limit the accuracy of RRP measurements obtained using these methods. These caveats include: (i) hypertonic sucroseevoked RRP measurements are critically dependent on the speed at which the sucrose is delivered to the cell and the precise mechanism of operation remains poorly defined; (ii) the accuracy of RRP estimates calculated by back-extrapolation of cumulative EPSCs evoked during high frequency action potential trains is sensitive to both dynamic changes in release probability during the stimulus train (i.e. short-term plasticity) and RRP refilling (i.e. calciumdependent priming); and (iii) postsynaptic responses report vesicle fusion indirectly and are consequently sensitive to dynamic changes in receptor properties (i.e. sensitization or saturation). Alternative approaches have been developed directly assay vesicle fusion. For example, the measurement of presynaptic capacitance evoked during the application of depolarizing voltage steps elicits dynamic changes in presynaptic membrane surface area resulting from evoked exo- and endocytosis (Delvendahl et al., 2016; Neher and Marty, 1982). However, this technique is only applicable to very large presynaptic boutons and is unable to discriminate concomitant exo- and endocytic processes during a depolarizing pulse. Alternatively, various optical approaches have been developed to report vesicle fusion, including lipid soluble fluorescent dyes (Rizzoli and Betz, 2004; Schikorski and Stevens, 2001), $\mathrm{pH}$-sensitive fluorescent reporters fused to vesicular proteins (Ariel and Ryan, 2010; Ariel et al., 2013), and fluorescent membrane proteins engineered to report glutamate release (Helassa et al., 2018; Oertner et al., 2002).

\subsubsection{Synaptic vesicle priming and exocytosis}

To generate the RRP, a complex molecular machinery is required to organize synaptic vesicles at the presynaptic membrane, including priming and membrane fusion proteins. Munc13 and calcium-dependent secretion activator (CAPS) priming proteins are essential for generating a functionally and molecularly primed pool of fusion-competent vesicles (Augustin et al., 1999; Imig et al., 2014; Jockusch et al., 2007; Varoqueaux et al., 2002). On the molecular level, vesicular priming requires a coordinated interaction with select core components of the exocytotic machinery, namely the neuronal soluble $\mathrm{N}$-ethylmaleimide-factor attachment protein receptors (SNARES). SNARE proteins comprise the target SNARE (tSNARE) proteins syntaxin-1 and SNAP-25 on the plasma membrane and the vesicular SNARE (VSNARE) 
synaptobrevin (Bennett et al., 1992; Link et al., 1992; Schiavo et al., 1992; Sollner et al., 1993). Upon a Munc13-mediated switch from a closed to an open conformation, syntaxin-1 interacts with SNAP-25 to preassemble a t-SNARE acceptor complex at active zone release sites (Ma et al., 2011). Upon arrival of a synaptic vesicle, the vSNARE synaptobrevin binds the tSNARE acceptor complex to form a tight, ternary SNARE complex (Fasshauer et al., 1997, 2002; Hatsuzawa et al., 2003). Whereas partial assembly of the SNARE complex is initially sufficient to dock the vesicle to the plasma membrane (Imig et al., 2014), it is the "zippering" together of the vSNARE and tSNAREs to form the final ternary SNARE complex structure which provides enough energy to drive the fusion of the vesicular and plasma membrane lipid bilayers (Hanson et al., 1997; Jahn et al., 2003; Lin and Scheller, 1997; Sollner et al., 1993).

\subsubsection{Molecularly primed synaptic vesicles are morphologically docked}

Genetic deletion of Munc13 priming proteins (Aravamudan et al., 1999; Augustin et al., 1999;

Richmond et al., 1999; Siksou et al., 2009a; Varoqueaux et al., 2002; Weimer et al., 2006) or components of the SNARE complex (Arancillo et al., 2013; Bronk et al., 2007; Schoch et al., 2001; Washbourne et al., 2002) results in absolute loss of or severe deficits in neurotransmission, respectively. Corresponding ultrastructural analyses combining highpressure cryofixation and electron tomography revealed docking deficits in Schaffer collateral synapses lacking either Munc13s, CAPSs, sytaxin-1, synaptobrevin-2, or SNAP-25 (Imig et al., 2014; Siksou et al., 2009a). As loss of individual SNARE proteins and priming factors severely affect both functional vesicle priming and morphological vesicle docking, the authors concluded that priming and docking are functional and morphological representations of the same process, specifically partial SNARE complex assembly is mediated by Munc13s and CAPSs priming molecules (Imig et al., 2014). Additional converging lines of evidence indicating that functionally primed vesicles are detected at the ultrastructural level as vesicles docked or in close physical contact with the active zone membrane include: (i) the number of docked vesicles correlates closely with the number of vesicles predicted to fuse in response to stimuli triggering the release of all fusion competent vesicles (Schikorski and Stevens, 1997); and (ii) optogenetically and electrically evoked action potentials selectively deplete the docked pool of vesicles (Kusick et al., 2020; Watanabe et al., 2013a).

Moreover, the discovery that synapses from genetic null mutants of Munc13 priming proteins or components of the SNARE complex accumulate vesicles within 5-10 nm of the active zone 
membrane provided evidence of morphologically distinct steps upstream of the docking/priming process (Imig et al., 2014; Siksou et al., 2009a). These findings appear consistent with the aforementioned data obtained by cryo-electron tomographic analyses of frozen-hydrated synaptosomes, which revealed structural filaments ("tethers") approximately $10 \mathrm{~nm}$ in length linking vesicles to the active zone membrane (FernándezBusnadiego et al., 2010, 2013).

Taken together, these findings indicate that morphologically docked vesicles fulfill at least the molecular requirements to fuse in response to vesicle depleting stimuli, such as hypertonic sucrose. It should however be considered that in response more physiologically relevant stimuli, such as single action potentials, only a subset of these docked and primed vesicles are likely to fuse. Moreover, synapse-specific properties that determine the size of this fusing subset critically determine how the efficacy of transmitter release is dynamically, or plastically, changed during repetitive stimulation.

\subsubsection{Release probability and short-term plasticity}

Despite the fact that many molecular components of the neurotransmitter release machinery are highly conserved between different synapse types, a remarkable range of functional synaptic behaviors are displayed depending on the synapse type or brain region in question (Südhof, 2012). Two important, and inherently linked, presynaptic parameters that contribute to this functional heterogeneity are synaptic release probability and short-term plasticity.

Synaptic release probability describes the likelihood that a given synaptic vesicle will fuse upon action potential arrival in the presynaptic terminal (Neher, 2015). The closer the release probability is to 1 , the more likely it is that a release-ready vesicle fuses during an action potential. Multiple factors can influence the release probability of a synapse, including the number of available fusion-competent vesicles (i.e. the size of the RRP) (Imig et al., 2014; Varoqueaux et al., 2005), the physical distance separating VGCCs from the vesicular calcium sensor (i.e. the coupling distance) (Chen et al., 2015; Rebola et al., 2019; Vyleta and Jonas, 2014), the geometrical arrangement of VGCCs at the active zone membrane (Keller et al., 2015; Miki et al., 2017; Rebola et al., 2019), the type and sensitivity of the vesicular calcium sensor (Chen et al., 2015; Fernández-Chacón et al., 2001; Jackman et al., 2016), and the intrinsic properties of the vesicle related to the state of its release machinery (Cano et al., 
2012). The notion of heterogeneous vesicular release probabilities within the RRP, however, remains controversial (Neher, 2015).

Synapse-specific differences in release probability shape their distinct short-term plasticity characteristics. Short-term plasticity, the alteration of synaptic strength upon repetitive stimulation, was first observed in the form of paired pulse facilitation in the frog neuromuscular junction and paired pulse depression in the cat neuromuscular junction (Eccles et al., 1941). Eccles and colleagues found that the endplate potential, the postsynaptic response in a muscle fiber, in the frog neuromuscular junction increased after two closely spaced stimuli and that this effect diminishes as the interstimulus interval, the time between two subsequent stimuli, increases (Eccles et al., 1941). Conversely, the cat neuromuscular junction undergoes paired-pulse depression in which the second endplate potential is smaller than the first (Eccles et al., 1941). Typically, synapses that undergo paired-pulse facilitation have a low initial release probability, such that more release-ready vesicles remain to fuse in response to subsequent stimuli and associated elevations in presynaptic calcium. Conversely, synapses with higher release probability release a greater proportion of vesicles during the initial stimulus, so that fewer vesicles remain to fuse in response to a second closely-spaced stimulus, thus leading to paired-pulse depression (reviewed in Jackman and Regehr, 2017).

Multiple factors contribute to dynamic changes in synaptic transmission efficacy and thereby to mechanisms mediating short-term plasticity. Short-term plasticity is postulated to be an important mechanism for the forming and processing of memory in the hippocampus (Neves et al., 2008). These factors include: action potential broadening (Geiger and Jonas, 2000); VGCC-vesicular calcium sensor coupling distance (Eggermann et al., 2012; Nakamura et al., 2018; Vyleta and Jonas, 2014); calcium sensors (Fernández-Chacón et al., 2001; Jackman et al., 2016); endogenous presynaptic calcium buffers (Blatow et al., 2003; Dumas et al., 2004; Müller et al., 2005; Vyleta and Jonas, 2014); and the availability of fusion competent synaptic vesicles (Imig et al., 2014; Miki et al., 2020; Siksou et al., 2009a; Südhof and Rizo, 2011).

Calcium influx is altered by a broadening of the action potential spike, which in turn extends the depolarization at the terminal and the number of open VGCCs. Action potential broadening has been studied in the hippocampal mossy fiber synapse where increased activity causes a broadening of the action potential and enhancement of synaptic transmission (Geiger and Jonas, 2000). Geiger and Jonas demonstrated that activity- 
dependent inactivation of potassium channels causes a broadening of the action potential spike (Geiger and Jonas, 2000). During spike broadening, VGCCs are open for longer periods of time, leading to increased presynaptic calcium concentrations and a concomitant enhancement of synaptic vesicle fusion (Geiger and Jonas, 2000).

Although the length of time a calcium channel is open during an action potential can modulate synaptic transmission, the distance between calcium channels and sensors located on synaptic vesicles is also important for synaptic efficacy. By loading presynaptic boutons with calcium chelators with different binding kinetics, the distance of synaptic vesicles to VGCCs can be estimated based on the degree synaptic transmission is reduced (Chen et al., 2015; Eggermann et al., 2012; Vyleta and Jonas, 2014). For example, the coupling distance of fastreleasing synaptic vesicles in the calyx of Held has been estimated to be approximately $16 \mathrm{~nm}$ (Chen et al., 2015), whereas a looser coupling of approximately $70 \mathrm{~nm}$ has been postulated for hippocampal mossy fiber synapses (Vyleta and Jonas, 2014). In another study, coupling distance was attributed to release probability in cerebellar stellate and granule cells, in which neurons with higher release probability had a tighter coupling distance than facilitating neurons (Rebola et al., 2019).

Another mechanism of facilitation is buffer saturation (Jackman and Regehr, 2017). Buffer saturation is caused by endogenous calcium-binding molecules buffering out free calcium ions upon calcium influx at the start of repeated stimulation (Jackman and Regehr, 2017). The remaining few free calcium ions trigger the fusion of few synaptic vesicles. Upon the arrival of a subsequent action potential, the endogenous buffers remain saturated, and more free calcium is available to trigger fusion of more synaptic vesicles (Jackman and Regehr, 2017). For the buffer saturation model to contribute to synaptic facilitation, a combination of highaffinity calcium buffers, high concentrations of calcium buffers, and relatively large distances between the calcium channels and sensors is required (Jackman and Regehr, 2017). For example, hippocampal mossy fiber synapses have a fast-acting calcium buffer, calbindin, with a high affinity for calcium that can rapidly buffer free calcium during a single action potential (Eggermann et al., 2012; Nägerl et al., 2000). Consequently, mossy fiber synapses exhibit short-term facilitation and a low release probability (Kawamura et al., 2004; Salin et al., 1996; Toth et al., 2000; Vyleta and Jonas, 2014). Other endogenous calcium buffers, such as 
calmodulin, are also found in mossy fiber boutons (Chamberland et al., 2018; Salin et al., 1996; Xia et al., 1991).

The type of calcium sensor residing on synaptic vesicles, such as synaptotagmins (Craxton, 2010; Südhof, 2002), may also contribute to short-term facilitation. While synaptotagmin-1 and synaptotagmin-2 isoforms are well-known calcium sensors for synaptic vesicle fusion, they contribute primarily to the fast component of transmitter release, likely on the first action potential during a series of action potentials (Brandt et al., 2012; Hui et al., 2005). Another calcium sensor, synaptotagmin-7, has very high calcium affinity, but slow disassociation kinetics as determined through in vitro studies (Brandt et al., 2012; Hui et al., 2005) and has been shown to contribute to facilitation in Schaffer collateral and mossy fiber synapses (Jackman et al., 2016). The presence of an additional calcium sensor with properties similar to synaptotagmin-7 could be a later-phase or asynchronous sensor involved in synaptic vesicle fusion in the second, facilitating pulse (Hui et al., 2005).

Lastly, and of particular importance to the motivation to perform this study, the availability of fusion-competent, docked synaptic vesicles may contribute to the functional heterogeneity of synapses (Dobrunz, 2002; Dobrunz and Stevens, 1997; Schikorski and Stevens, 1997). Based on the assumption that morphologically docked vesicles overlap with the functional RRP (Imig et al., 2014; Kusick et al., 2020; Schikorski and Stevens, 1997; Siksou et al., 2009a; Watanabe et al., 2013a) many previous studies have attempted to elucidate whether the availability of docked synaptic vesicles contribute to synapse-specific differences in synaptic release probability and plasticity characteristic (Eltes et al., 2017; Holderith et al., 2012; Millar et al., 2002; Xu-Friedman et al., 2001). These studies failed to come to a strong consensus, possibly due to the variety of organisms, brain regions and synapse types examined, and variations in the methodological approaches used.

As an example, a comparative analysis of climbing fiber (low release probability, facilitating) and parallel fiber (high release probability; depressing) synapses in the cerebellum of perfusion-fixed rats found no difference in the availability of docked synaptic vesicles analyzed by three-dimensional (3D) serial section EM (Xu-Friedman et al., 2001). A study of associational/commissural synapses onto cornu ammonis area 3 (CA3) pyramidal neurons in the hippocampus of chemically-fixed acute rat slices found that synapses with low release probability had fewer docked synaptic vesicles than those with high release probability 
analyzed by 3D serial EM (Holderith et al., 2012). However, excitatory CA3 pyramidal synapses onto metabotropic glutamate receptor type1 $\alpha$-positive interneurons (facilitating synapses) harbored fewer docked vesicles than synapses onto parvalbumin-positive interneurons (depressing synapses) in the hippocampus of perfusion-fixed rats analyzed by electron tomography (Eltes et al., 2017). Conversely, a comparative ultrastructural study of phasic (depressing) and tonic (facilitating) motor neurons found tonic motor neurons harbored more docked synaptic vesicles than phasic motor neurons of the main leg extensor muscle of freshwater crayfish chemically fixed and analyzed by 3D serial EM (Millar et al., 2002). Taken together, these studies do not come to a consensus regarding the number of morphologically available synaptic vesicles in shaping synaptic functional properties.

To understand these conflicting findings, I address the same question in this thesis using stateof-the-art methodological approaches introduced later in this section to perform a comparative ultrastructural analysis of two extensively characterized excitatory synapses in the hippocampal formation, mossy fiber-CA3 and Schaffer collateral synapses. As indicated above, many factors have been implicated as mechanisms contributing to mossy fiber shortterm facilitation (Chamberland et al., 2014; Dumas et al., 2004; Geiger and Jonas, 2000; Jackman et al., 2016; Vyleta and Jonas, 2014). However, the relationship between the organization of synaptic vesicles and the plasticity characteristics of this synapse remains unclear. This information is ultimately required to fully understand mossy fiber synapse function, both at the level of the synapse and in the context of the hippocampal network. 

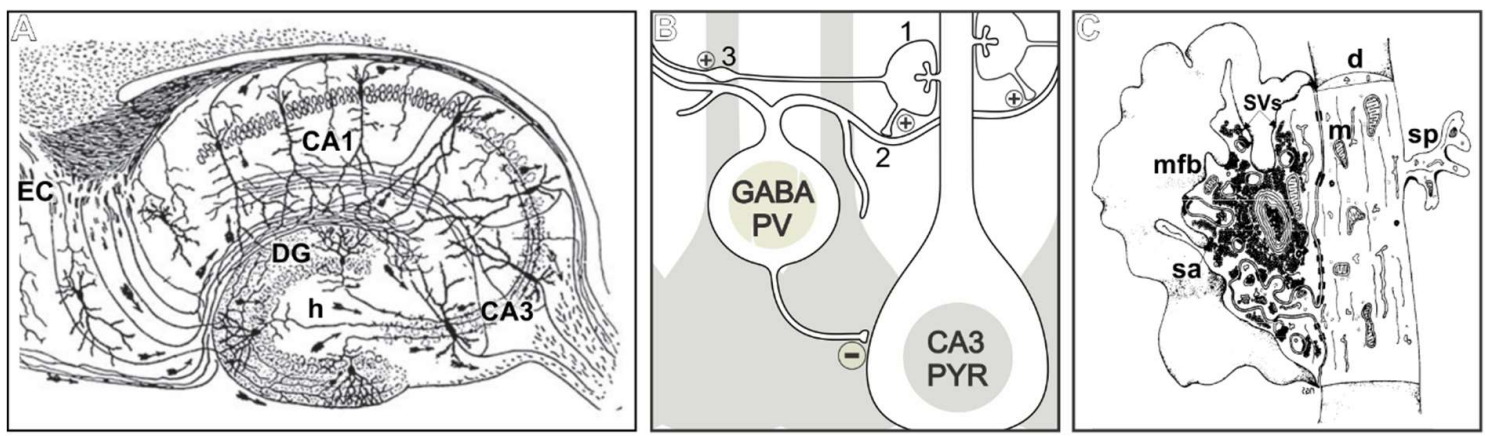

Figure 3. Hippocampal network connectivity and the mossy fiber pathway.

(A) Connectivity of neurons in the hippocampus as originally described by Ramon y Cajal. Granule cells with cell bodies located in the dentate gyrus (DG) send axonal projections to the CA3 where they form three types of synaptic connections. (B) Illustration of synaptic connections formed by granule cell mossy fibers in the hippocampus. Mossy fibers form 1) mossy fiber boutons onto the primary dendrite and thorny spine excrescences of CA3 pyramidal neurons; 2) filopodial extensions from the mossy fiber boutons; and 3) axonal en passant boutons onto inhibitory interneurons that feedforward onto CA3 pyramidal neurons. (+) and (-) symbols represent excitatory glutamatergic and inhibitory GABAergic synapses, respectively. (C) Depiction of a mossy fiber bouton $(\mathrm{mfb})$ and complex thorny spine excrescences ( $\mathrm{sp}$ ) of CA3 pyramidal neurons adapted from Amaral and Dent, 1981. Abbreviations: CA1/CA3, cornu ammonis area 1 and 3; DG, dentate gyrus; EC, entorhinal cortex; $h$, hilus; d, dendrite; m, mitochondria; mfb, mossy fiber bouton; sa, spine apparatus; $s p$, thorny spine excrescence; SVs, synaptic vesicles. Permission \& Rights: (A) Adapted from Nicoll and Schmitz, 2005 through Copyright Clearance Center with license number 4786480011593. (C) Adapted from Amaral and Dent, 1981 through Copyright Clearance Center with license number 4786540090843.

\subsection{The role of functional heterogeneity in brain circuits: the hippocampus}

The hippocampal formation is the brain area in which information from the cortex is processed for storage in the form of memory (Jarrard, 1993). Memory formation relies on a well-described anatomical circuit of excitatory neural connections called the tri-synaptic pathway (Andersen et al., 1966). The tri-synaptic pathway is composed of three different pathways, the perforant, the mossy fiber, and the Schaffer collateral pathways. The perforant pathway comprises axonal projections from the entorhinal cortex $(\mathrm{EC})$ that form excitatory synapses onto the granule cells of the dentate gyrus (DG) (Figure 3 A) (Blackstad and Kjaerheim, 1961). The second synaptic connection is the mossy fiber pathway where granule cells send axonal projections to the CA3 where they form giant, excitatory mossy fiber synapses onto CA3 pyramidal neurons (Figure 3 A) (Blackstad and Kjaerheim, 1961). Finally, in the Schaffer collateral pathway, CA3 pyramidal neurons send axonal projections to the CA1 where they form synaptic connections with pyramidal neurons (Figure 3 A) (Blackstad and Kjaerheim, 1961). Outgoing axonal projections run from the CA1 to layer $V$ neurons in the EC via subiculum (Ceccom et al., 2014). 


\subsubsection{Mossy fiber pathway}

Although a simplistic anatomical view is helpful to understand the "flow" of information through the hippocampus, a deeper consideration of local circuitry, including feedforward and feedback loops, and of synapse-specific functional properties and plasticity characteristics is ultimately required to understand or predict the behavior of complex neuronal networks. The hippocampal mossy fiber projection serves as an excellent example of how such complexity shapes its role within the tri-synaptic pathway.

Mossy fiber synapses are generated from granule cell axonal projections to the stratum lucidum of the CA3 and establish excitatory synaptic connections with pyramidal neurons along the first $100 \mu \mathrm{m}$ of the primary dendrites via large en passant boutons (Henze et al., 2000). One granule cell forms 15-18 large mossy fiber boutons (Amaral and Dent, 1981) and each pyramidal neuron in the CA3 is innervated by approximately 50 mossy fiber boutons (Amaral et al., 1990). Demonstrative of the mossy fiber projection's unique level of target specificity, small granule cell axonal varicosities and filopodial extensions emerging from the bouton form synapses onto local inhibitory interneurons that target the soma of CA3 pyramidal cells (Figure 3 B). In total, each granule cell axon forms approximately 150 synapses onto inhibitory interneurons. Whereas large synapses formed by large mossy fiber boutons exhibit very low initial release probability and profound frequency facilitation (Lawrence et al., 2004; Salin et al., 1996), synapses formed by small varicosities and filopodial extensions depress in during repetitive firing (Toth et al., 2000). Based on this combination of anatomical and physiological properties, the net effect of granule cell firing at basal frequencies is one of feedforward inhibition of CA3 pyramidal cells (Acsády et al., 1998). In contrast, during elevated firing rates, the profound frequency facilitation (Salin et al., 1996) exhibited by mossy fiber-CA3 synapses overcomes this feedforward inhibition and fulfills a "conditional detonator" function with a profound influence on the excitability of postsynaptic CA3 pyramidal cells (Henze et al., 2002a).

\subsubsection{Structural comparison}

The low initial release probability of the mossy fiber-CA3 synapse is perhaps unexpected considering ultrastructural features including the total number of synaptic vesicles and active zone release sites harbored within individual giant mossy fiber boutons (Amaral and Dent, 1981; Chicurel and Harris, 1992; Henze et al., 2000; Rollenhagen et al., 2007; Wilke et al., 
2013). Mossy fiber boutons are several microns in diameter and filled with tens of thousands $(\sim 20,500)$ of synaptic vesicles (Figure 3 C; Table 1; Chicurel and Harris, 1992; Rollenhagen et al., 2007). These boutons are easily distinguishable in ultrastructural studies due to their large size as well as the presence of multiple synaptic contacts onto the CA3 pyramidal neuron (Table 1; Chicurel and Harris, 1992; Rollenhagen et al., 2007). Mossy fiber boutons form synapses onto specialized, multi-headed spines on CA3 pyramidal neurons called thorny excrescences (Figure 3 C; Table 1; Chicurel and Harris, 1992). Unlike classically described dendritic spines, thorny excrescences tend to contain many organelles, including microtubules, multivesicular bodies, and spine apparatuses (Chicurel and Harris, 1992).

Presynaptically, giant mossy fiber boutons are characterized by a relative abundance of mitochondria, microtubules, and mutivesicular bodies (Amaral and Dent, 1981; Chicurel and Harris, 1992; Rollenhagen et al., 2007). In addition to the small clear-core vesicles typical of glutamatergic synapses, mossy fiber synapses also harbor clear-core synaptic vesicles of considerably larger dimensions (Henze et al., 2002b; Laatsch and Cowan, 1966) and densecore vesicles (DCVs) (Amaral and Dent, 1981; Rollenhagen et al., 2007). DCVs are characterized in electron micrographs as large vesicles (70-100 nm in diameter) with electrondense cores (Amaral and Dent, 1981).

The origin and functional implications of giant clear-core vesicles remain largely unknown, although it has been previously postulated that they contribute to glutamatergic signaling between granule cells and postsynaptic CA3 pyramidal cells (Henze et al., 1997). Based on the

Table 1. Comparative morphologies of Schaffer collateral and mossy fiber synapses from ultrastructural studies.

\begin{tabular}{|c|c|c|c|}
\hline & Averages & $\begin{array}{c}\text { Schaffer collateral } \\
\text { synapses }\end{array}$ & Mossy fiber synapses \\
\hline \multirow[t]{4}{*}{ Presynapse } & Bouton volume $\left(\mu \mathrm{m}^{3}\right)$ & $0.11^{1}$ & $7-8^{2}$ \\
\hline & Number of SVs & $223^{1}$ & $\sim 20,400^{2}$ \\
\hline & Number of AZs per bouton & $1^{1}$ & $29.75^{2}$ \\
\hline & AZ area $\left(\mu m^{2}\right)$ & $\sim 0.03^{3}$ & $0.12^{2}$ \\
\hline \multirow[t]{3}{*}{ Postsynapse } & Spine volume $\left(\mu \mathrm{m}^{3}\right)$ & $0.03^{1}$ & $0.13-1.83^{2}$ \\
\hline & Spine area $\left(\mu \mathrm{m}^{2}\right)$ & $0.63^{1}$ & $16-23^{2}$ \\
\hline & Total PSD area $\left(\mu \mathrm{m}^{2}\right)$ & $0.069^{1}$ & $1-3^{2}$ \\
\hline \multicolumn{4}{|c|}{ Abbreviations: AZ, active zone, PSD, postsynaptic density; SVs, synaptic vesicles. } \\
\hline \multicolumn{4}{|c|}{${ }^{1}$ Harris and Stevens, $1989 ;{ }^{2}$ Rollenhagen et al., 2007; ${ }^{3}$ Shepherd and Harris, 1998} \\
\hline
\end{tabular}


large lumenal volume (i.e. potential neurotransmitter capacity) and the proximity of giant mossy fiber boutons to the cell bodies of CA3 pyramidal cells, the fusion of such large vesicles in this scenario could be expected to profoundly influence the excitability of the postsynaptic neuron. Despite this interesting hypothesis, giant vesicles remain enigmatic and many important questions are still to be addressed. For example: i) Do giant vesicles dock in physical contact with active zone membranes and are they molecularly equipped to be primed for fusion? ii) Through what mechanisms are giant vesicles formed and are they regulated by synaptic activity?

Although the mossy fiber pathway uses glutamate as a primary neurotransmitter, other messengers such as neuropeptides (Chavkin et al., 1983; Henze et al., 2000; McQuiston and Colmers, 1996; Salin et al., 1995; Sherwood and Lo, 1999; Weisskopf et al., 1993), zinc (Lavoie et al., 2011), and adenosine triphosphate (ATP) (Henze et al., 2000; Loewen et al., 1992; Yamamoto et al., 1993), modulate synaptic function. Neuropeptides produced in hippocampal granule cells and trafficked to mossy fiber boutons (Henze et al., 2000), include, but are not limited to, brain-derived neurotrophic factor (BDNF), enkephalins, dynorphin, and neuropeptide $Y$ (Chavkin et al., 1983; Henze et al., 2000) These relatively large peptide signaling molecules (3-36 amino acids; compared to single amino acids like glutamate and GABA) are synthesized in the rough endoplasmic reticulum within the cell soma, and transported to the Golgi apparatus (Gondré-Lewis et al., 2012). Once at the Golgi apparatus, they are packaged into DCVs (Commons and Milner, 1995; Dieni et al., 2012, 2015) and transported along microtubules via anterograde axonal transport to presynaptic boutons (Gondré-Lewis et al., 2012). Upon DCV fusion, neuropeptides can affect synaptic transmission via actions on both presynaptic and postsynaptic targets (Chavkin et al., 1983; McQuiston and Colmers, 1996; Salin et al., 1995; Sherwood and Lo, 1999; Weisskopf et al., 1993).

In contrast to the intriguing morphology of hippocampal mossy fiber synapses, Schaffer collateral synapses represent prototypical examples of small, glutamatergic synapses. On the ultrastructural level, Schaffer collateral synapses are visualized as small axonal varicosities filled with several hundred synaptic vesicles (Harris and Stevens, 1989) that cluster at asymmetric synaptic contacts onto postsynaptic dendritic spines of CA1 pyramidal cells in the stratum radiatum (Figure 1 B; Table 1; Gray, 1959; Harris and Stevens, 1989). Schaffer collateral synapses typically form one synapse onto one spine, however, on occasion one 
presynaptic bouton can contact multiple spines (Table 1; Harris and Stevens, 1989). Other organelles, such as DCVs, microtubules, and mitochondria are also present in the Schaffer collateral presynaptic compartment (Harris and Stevens, 1989). The postsynaptic spine lacks organelles with the exception of the occasional spine apparatus (appearing in approximately $23 \%$ of mature CA1 spines), a lamellar stack of smooth-endoplasmic reticulum membranes with electron-dense F-actin filaments between the folds of each lamella (Capani et al., 2001; Spacek and Harris, 1997). 


\subsection{Schaffer collateral and mossy fiber synapses}

\subsubsection{Functional differences between synapses}

The two synapses I focus on in this study have well-characterized differences in synaptic efficacy. Both synapse types exhibit paired pulse facilitation indicating a low release probability. However, the release probability of Schaffer collateral synapses is heterogeneous in acute slices when using an external fluorescent indicator that binds glutamate (Helassa et al., 2018; Oertner et al., 2002), and is considerably higher than in mossy fiber synapses (Lawrence et al., 2004). For comparison, Schaffer collateral synapses facilitate for the first few action potentials and then depress during high frequency stimulation (Dobrunz and Stevens, 1997, 1999; Helassa et al., 2018; Jackman et al., 2016; Oertner et al., 2002). In comparison, mossy fiber synapses exhibit strong frequency facilitation (Table 2; Jackman et al., 2016; Salin et al., 1996). Frequency facilitation is the reversible enhancement of synaptic transmission at low frequency stimulation (Salin et al., 1996). For example, the amplitude of mossy fiberdriven EPSCs increases by up to $300 \%$ of baseline when the frequency of the stimulus changes from $0.05 \mathrm{~Hz}$ to $0.2 \mathrm{~Hz}$ in acute guinea pig hippocampal slices (Salin et al., 1996).

Synaptic ultrastructure-function relationships can also be probed in individual synapses by modulating their functional state. Various pharmacological manipulations that target specific presynaptic molecules have been utilized in past studies to induce changes in neurotransmitter release efficacy. For example, mossy fiber synaptic transmission is strongly potentiated following the bath application of either tetraethylammonium (TEA) (Suzuki and Okada, 2008; Zhao et al., 2012a) or forskolin (Weisskopf et al., 1994). Although TEA and forskolin both act via presynaptic mechanisms to induce a prolonged state of enhanced mossy

Table 2. Functional properties of Schaffer collateral and mossy fiber synapses.

\begin{tabular}{|c|c|c|}
\hline Averages & Schaffer collateral synapses & Mossy fiber synapses \\
\hline $\begin{array}{c}\text { Readily releasable pool of } \\
\text { vesicles }\end{array}$ & 10 per synapse ${ }^{1}$ & $400-1400$ per bouton ${ }^{5,6}$ \\
\hline $\begin{array}{c}\text { Morphological RRP estimates } \\
\text { (per AZ) }\end{array}$ & $10-12^{2}$ & $36.6^{5}$ \\
\hline Release probability & $0.2-0.6^{3}$ & $<0.1^{7}$ \\
\hline $\begin{array}{l}\text { Short-term plasticity } \\
\text { characteristics }\end{array}$ & Mild-facilitation ${ }^{4}$ & Strong frequency facilitation ${ }^{8}$ \\
\hline \multicolumn{3}{|c|}{$\begin{array}{l}{ }^{1} \text { Stevens and Tsujimoto, } 1995 ;{ }^{2} \mathrm{Imig} \text { et al., } 2014 ;{ }^{3} \text { Oertner et al., } 2002 ;{ }^{4} \text { Jackman et al., } 2016 ;{ }^{5} \text { Rollenhagen } \\
\text { al., 2007; }{ }^{6} \text { Midorikawa and Sakaba, 2017; }{ }^{7} \text { Vyleta and Jonas, 2014; }{ }^{8} \text { Salin et al., 1996. Abbreviation: AZ, } \\
\text { active zone. }\end{array}$} \\
\hline
\end{tabular}


fiber transmission referred to as chemical long-term potentiation (LTP), the two drugs target different molecules. Whereas TEA blocks presynaptic potassium channels, thereby broadening the action potential spike, prolonging VGCC channel opening, and increasing the presynaptic influx of calcium (Ishikawa et al., 2003; Suzuki and Okada, 2008), forskolin activates adenylate cyclase 1 (AC1), which enhances mossy fiber synaptic transmission via increased presynaptic cyclic adenosine monophosphate (cAMP) (Barovsky et al., 1984; Weisskopf et al., 1994). When calcium enters the presynaptic terminal via VGCCs, it triggers synaptic vesicle fusion and also acts as a second messenger to activate AC1 (Villacres et al., 1998), which converts ATP into CAMP (Chavez-Noriega and Stevens, 1994). Although the presynaptic signaling cascades and molecular mechanisms underpinning CAMP-dependent enhancement of mossy fiber synaptic transmission have been partially elucidated (Henze et al., 2000; Huang et al., 1994a; De Rooij et al., 1998; Trudeau et al., 1996; Tzounopoulos et al., 1998; Villacres et al., 1998; Weisskopf et al., 1994), it remains unclear precisely how they ultimately manifest as changes in release probability at active zone release sites. For example, both the enzyme protein kinase A (PKA) (Weisskopf et al., 1994) and exchange protein directly activated by CAMP (Epac) (Fernandes et al., 2015) have been implicated as downstream targets of cAMP signaling involved in enhancing presynaptic mossy fiber release probability. Although several candidate molecules have been postulated to act as downstream effectors in these pathways (Castillo et al., 2002; Cho et al., 2015; Fykse et al., 1995; Kaeser-Woo et al., 2013; Lonart and Sudhof, 1998), their potential influence on the structural organization of active zone release sites remains to be investigated in the context of enhanced transmitter release probability.

Application of the metabotropic glutamate receptor type 2 (mGluR2) agonist ( $2 S, 1^{\prime} R, 2^{\prime} R, 3^{\prime} R$ )2-(2,3-dicarboxy-cyclopropyl)glycine (DCG-IV), decreases mossy fiber presynaptic cAMP levels (Chen et al., 2001; Huang et al., 2002). DCG-IV acts upon presynaptically expressed mGluR2 receptors, causing a decrease in evoked and spontaneous release of synaptic vesicles at mossy fiber synapses (Kamiya and Ozawa, 1999; Kamiya et al., 1996). Consequently, DCG-IV is commonly used in electrophysiological experiments to demonstrate that EPSCs recorded from CA3 pyramidal cells are of mossy fiber origin (Breustedt et al., 2010; Brockmann et al., 2019; Castillo et al., 2002; Galimberti et al., 2006; Kamiya et al., 1996; Nicoll and Schmitz, 2005). In the hippocampus, mGluR2 expression is specific to the presynaptic terminals of 
mossy fiber synapses (Ikeda et al., 1995). DCG-IV suppresses synaptic transmission by reducing presynaptic calcium influx as well as by acting on the vesicular release machinery, however the mechanism of action is not fully understood (Kamiya and Ozawa, 1999).

In summary, the functional state of hippocampal mossy fiber synapses is highly sensitive to presynaptic cAMP levels (Fernandes et al., 2015; Villacres et al., 1998; Weisskopf et al., 1994), which can be bidirectionally manipulated by forskolin or DCG-IV treatment. My objective is to exploit these pharmacologically induced changes in mossy fiber transmission efficacy to examine a potential link between CAMP-dependent alterations in release probability and changes in the availability of morphologically docked synaptic vesicles at active zone release sites. This has not previously been investigated in hippocampal mossy fiber synapses.

\subsubsection{Correlating structure and function}

The main objective of this study is to examine the relationship between the availability of morphologically docked synaptic vesicles, thus the RRP, and release probability and synaptic strength. My decision to focus primarily on the hippocampal mossy fiber synapses was motivated by several factors. Most importantly, the mossy fiber-CA3 synapses is characterized by a very low release probability and strong frequency facilitation (Lawrence et al., 2004; Salin et al., 1996; Vyleta and Jonas, 2014; Vyleta et al., 2016). Moreover, both shortand long-term plasticity at mossy fiber synapses are regulated presynaptically (Salin et al., 1996; Toth et al., 2000; Zalutsky and Nicoll, 1990). Whereas the anatomical connectivity and gross presynaptic morphology of the mossy fiber-CA3 projection has been well characterized (Acsády et al., 1998; Chicurel and Harris, 1992; Rollenhagen et al., 2007; Sai et al., 2017; Wilke et al., 2013), the fine structural organization of mossy fiber active zones has not previously been scrutinized at a resolution permitting accurate discrimination of functionally distinct vesicle pools. Consequently, the relative contribution of active zone structural organization cannot be assessed in parallel with other postulated mechanisms (e.g. coupling distance, calcium buffer species, and action potential broadening) of mossy fiber release probability. Hippocampal mossy fibers are also of particular advantage for relating ultrastructural observations with recorded functional parameters. The large size of presynaptic mossy fiber boutons permits direct presynaptic capacitance recordings to be made during stepdepolarizations (Hallermann et al., 2003; Midorikawa and Sakaba, 2017). Thus, quantitative morphological estimates of docked and membrane-proximal vesicle pools obtained by 
electron tomography can be compared with functional RRP estimates (Hallermann et al., 2003; Midorikawa and Sakaba, 2017). Based on these factors, I was motivated to revisit the question of whether the functional status of a synapse can be predicted by the spatial organization of membrane proximal vesicles. My hypothesis is that an experimental approach combining organotypic hippocampal slice culture, rapid high-pressure freezing (HPF) cryofixation, and 3D electron tomography will provide a novel, and more accurate, perspective of synaptic ultrastructure-function relationships by circumventing methodological limitations associated with past studies (see Discussion section Methodological considerations). 


\subsection{Methodology}

\subsubsection{Hippocampal slice cultures}

Most studies of synaptic and network function rely on access to brain tissue either maintained in vitro or acutely prepared slices of isolated brain regions. Exhibiting the least complex neuronal circuitry, autaptically cultured neurons are isolated and grown on astrocyte islands, forcing them to makes synapses onto themselves (Bekkers and Stevens, 1991; Millet and Gillette, 2012). In contrast, continental, or dissociated, primary neuron cultures generated from dissociated brain tissue establish synaptic connections with one another in a culture dish (Harrison, 1910; Millet and Gillette, 2012). Although both these reduced complexity culture systems are advantageous for assessing fundamental functional synaptic properties, not all synapse types can be recapitulated in cultured monolayers and many plasticity characteristics exhibited by synapses in vivo are not comparably expressed in vitro (Mennerick and Zorumski, 1995).

Organotypic slice culture systems maintain a near-native network connectivity, such thereby circumventing, or greatly mitigating many of the aforementioned limitations (Debanne et al., 1996; Gähwiler, 1984; Galimberti et al., 2006; Humpel, 2016; Marchal and Mulle, 2004). Moreover, cultures can be used to maintain neural networks in animals with post-natal lethality and to identify specific synapses within the tissue due to stereotyped targeting within the circuit (Imig et al., 2014). Slices cultured according to the interface method are maintained at the gas-liquid interface on top of a semi-permeable membrane insert, which provides access to slice culture medium containing metabolic nutrients (Stoppini et al., 1991). Hippocampal slice cultures have been extensively used in ultrastructural studies (Galimberti et al., 2006; Imig and Cooper, 2017; Studer et al., 2014; Zhao et al., 2012b, 2012c, 2012a) since they contain synaptic networks with well-studied synaptic functional characteristics that differ from one another (Eltes et al., 2017; Holderith et al., 2012; Jackman et al., 2016; Nicoll and Schmitz, 2005; Salin et al., 1996). As the slice recovers from the trauma of sectioning, minor rearrangements of the can tissue occur, however the stereotypical cellular lamination of the hippocampus remains intact (Buchs et al., 1993). Possible changes in the network connectivity include an increase in synapse density in the stratum ratiatum of the CA1 as a compensatory mechanism due to the loss of other synaptic inputs from other brain areas (Muller et al., 1993). Granule cells have been found to sprout in hippocampal slice cultures 
and form excitatory inputs back to the granule cell layer (Coltman et al., 1995; Frotscher et al., 2006), however simply culturing more EC remedies sprouting (Coltman et al., 1995).

\subsubsection{Fixation methods}

\subsubsection{Aldehyde fixation}

Conventional tissue fixation methods have long included the use of aldehydes. Two types of aldehydes are frequently used for tissue fixation and ultrastructural studies. Paraformaldehyde (PFA) and glutaraldehyde (GA) are the most commonly used aldehydes, which fix tissue by cross-linking free amino acids (Bullock, 1984; Hopwood, 1969). Several structural artifacts have been attributed to aldehyde fixation protocols for EM, including deformation of subsynaptic organelles (Murk et al., 2003) and depletion of membraneproximal synaptic vesicle pools (Korogod et al., 2015; Smith and Reese, 1980). However, aldehydes can be used to fix large tissue volumes and has been particularly advantageous for large-scale ultrastructural studies (Korogod et al., 2015).

\subsubsection{High-pressure freezing}

A method called HPF (high-pressure freezing) uses the principle of super-cooling water under high pressures to cause water vitrification rather than ice crystallization and can improve the penetration depth of cryopreservation in biological tissues (Dahl and Staehelin, 1989; Kanno et al., 1975; Moor, 1987; Moor et al., 1980). HPF creates high pressure and rapid cooling at the same time by forcing liquid nitrogen on the top and bottom of a sample at a high velocity. To achieve ideal cryopreservation, pressure on the sample must reach 2000 bar before the temperature drops below $0^{\circ} \mathrm{C}$ (Dahl and Staehelin, 1989). If the timing of either parameter is off, this can lead to poor ultrastructural preservation in the form of either ice crystal damage, as in instances when temperature reached $0^{\circ} \mathrm{C}$ before pressure reaches 2000 bar, or pressure artifacts, as in instances when pressure increases before the temperature drops (Möbius et al., 2010). Both artifacts can only be assessed at the electron microscopic level. Potential caveats of HPF include, (i) sample dimensions limited to a size compatible with the carriers used for HPF (Dahl and Staehelin, 1989; Korogod et al., 2015), and (ii) the depth of ideal tissue cryopreservation depends on the water content of the sample which can be partially mitigated by the use of additional external cryoprotectants (Dahl and Staehelin, 1989). 
Fixation of tissue with HPF coupled with automated freeze substitution (AFS) can capture dynamic cellular processes in near-native conditions with little to no alteration to tissue ultrastructure. AFS is the process in which tissue can be infiltrated with solvents for ideal plastic polymers at very low temperatures to prevent unwanted alterations to cellular ultrastructure (Giddings, 2003). Once the tissue has been dehydrated and contrasted with heavy metal solutions such as osmium tetroxide $\left(\mathrm{OsO}_{4}\right)$ then the tissue can be infiltrated with plastic polymers such as Epon to embed for EM (Finck, 1960; Giddings, 2003).

\subsubsection{Electron microscopy}

\subsubsection{Transmission electron microscopy}

Transmission electron microscopes (TEMs) are an ideal way to investigate nano-scale structures in tissue samples. TEMs pass a beam of electrons perpendicular to and directly through a plastic-embedded sample to generate a magnified image in a viewing screen or camera below. In the field of neuroscience, EM has allowed many important ultrastructural observations of tissue samples. Transmission EM has been a key method in linking functional studies to ultrastructure. Typically, ultrathin sections 50-60 $\mathrm{nm}$ thick have been used to assess vesicle clustering in proximity to and docking at the presynaptic membrane. Membrane curvature, overlapping synaptic vesicle projections, and incomplete cross-sections of synaptic vesicles can all confound the accurate detection of docked and tethered synaptic vesicles in two-dimensional (2D) EM analyses (Imig and Cooper, 2017; Verhage and Sørensen, 2008). Limited z-resolution of 2D transmission EM can lead to an inaccurate assessment of ultrastructural measurements such as an average vesicle diameter of 33-36 nm from 2D ultrastructural studies (Harris and Sultan, 1995) versus $\sim 45 \mathrm{~nm}$ reported via 3D electron tomography (Imig et al., 2014). While the resolution in $\mathrm{x}$ and $\mathrm{y}$ dimensions can achieve resolutions less than $1 \mathrm{~nm}$, the z-resolution of transmission EM of ultrathin section is restricted by the mechanical limitations of ultramicrotomy (thinnest sections approximately $20 \mathrm{~nm}$-thick; Holderith et al., 2012). Furthermore, the accurate assessment of synaptic vesicle 

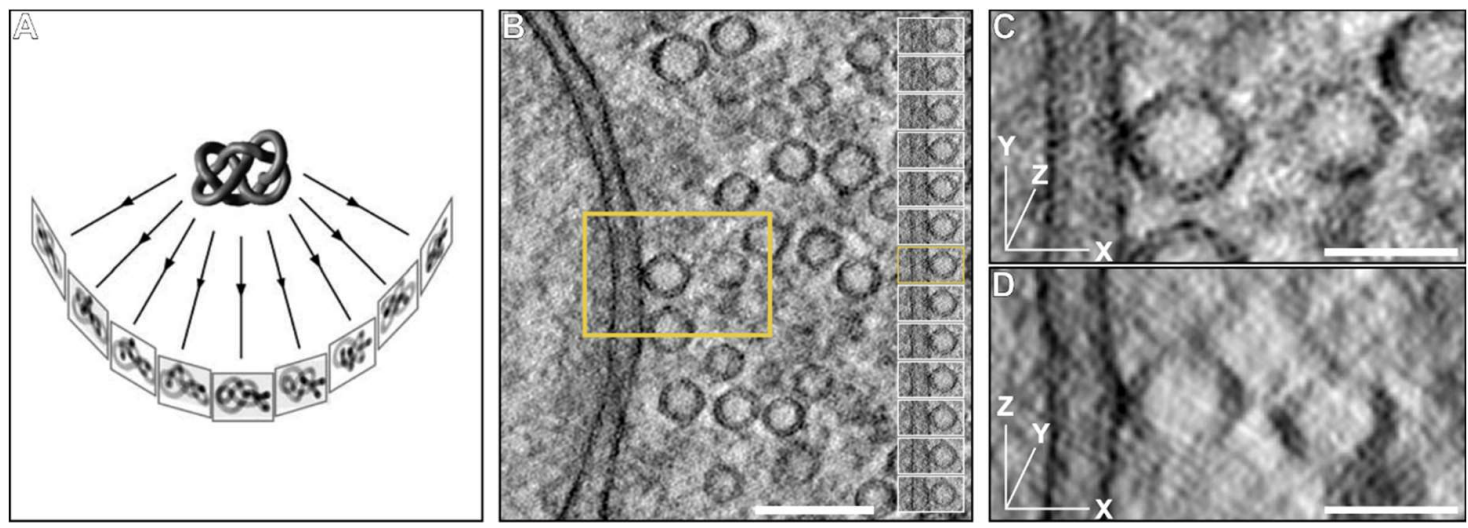

Figure 4. Electron tomography.

(A) Electron tomography works under the principle of reconstructing a three-dimensional object by taking a series of 2D images at many angles. For the electron tomography used in this study, specimens were tilted from $-60^{\circ}$ to $+60^{\circ}$ and $2 \mathrm{D}$ images acquired every $1^{\circ}$. Using a weighted pack-projection algorithm the series of $2 \mathrm{D}$ images are reconstructed into tomograms. (B) Slicer image of a reconstructed synaptic subvolume imaged at 30,000x magnification and binned 3x during reconstruction of the tomogram yielded isotropic voxel dimensions of $x, y$, and $z=1.6 \mathrm{~nm}$. Docked synaptic vesicles (yellow box and inset) could be accurately assessed in tomograms my moving through the z-stack to determine whether there was no measurable distance between synaptic vesicle and plasma membranes (inset: 13 consecutive sections of the reconstructed subvolume). (C-D) Higher magnification of docked synaptic vesicle from $B$ in $x, y$ (C) and $z, x$ (D) showing that this vesicle definitively fits the docking criteria set for this study. Scale Bars: $100 \mathrm{~nm}, \mathbf{B}$; 50 nm, C-D. Permission \& Rights: (A) Adapted from Lučić et al., 2005 with permission through Copyright Clearance Center with order number 1051526. (B-D) Adapted from Imig and Cooper, 2017 with permission through Copyright Clearance Center with license number 4877680012945.

docking requires a better z-resolution that can be achieved with electron tomography (Fernández-Busnadiego et al., 2010, 2013; Imig and Cooper, 2017; Siksou et al., 2009a, 2009b;

Stigloher et al., 2011; Vogl et al., 2015).

\subsubsection{Electron tomography}

The theoretical idea of using a set of projection images to generate a 3D reconstructed image was first proposed in the 1960s (Baumeister, 2002; De Rosier and Klug, 1968). Electron tomography works by creating projection images of a specimen, in the case of the present study, tissue embedded in plastic (Figure 4 A) (Baumeister, 2002). Projection images are created by tilting the specimen around an axis that sits perpendicular to the electron beam and taking 2D images incrementally through the entire tilt series (Figure $4 \mathrm{~A}$ ) (Baumeister, 2002). In my study, specimens were tilted from $-60^{\circ}$ to $+60^{\circ}$ and $2 \mathrm{D}$ images were taken every one degree (Figure 4; Kremer et al., 1996; Mastronarde, 2005). Raw image files are aligned and a weighted back-projection algorithm calculates a density map in order to reconstruct the final 3D tomogram (Figure 4 B) (Baumeister, 2002; Kremer et al., 1996; Mastronarde, 
2005). Limitations of electron tomography include the thickness of the section and the strength of the electron beam generated in the microscope (Baumeister, 2002). High-voltage electron microscopes create an electron beam powerful enough to penetrate the thicker sections required for specimens at high tilt angles. For example, at a tilt angle of $70^{\circ}, \mathrm{a}$ $200 \mathrm{~nm}$-thick section (at $0^{\circ}$ ) will be over $500 \mathrm{~nm}$-thick (Baumeister, 2002). At these extreme tilt angles, considerable scattering of the electron beam occurs and image quality drops (Baumeister, 2002). Depending on the imaging magnification, section thickness, camera used for image acquisition, and degree of image binning during tomogram reconstruction, the voxel dimensions and thus the resolution will vary. I achieved a voxel dimension of $1.554 \mathrm{~nm}$ by imaging $200 \mathrm{~nm}$-thick sections at 30,000x magnification and binning by 3 during tomogram reconstruction (see Materials and Methods; Figure 4 B-D). With electron tomography, I could more accurately measure synaptic vesicle diameters by finding the exact midpoint of a given synaptic vesicle and precisely measuring the distance between synaptic vesicles and the presynaptic membrane. Furthermore, tomograms can better reveal if a vesicle is morphologically docked or in a membrane proximal position, such as tethered synaptic vesicles within the synaptic subvolume (Figure $4 C$ and $D$ ). 


\subsection{Purpose of this study}

1. There is agreement in the field that the number of morphologically docked synaptic vesicles correlates with the RRP and that docking and priming are morphological and functional manifestations of the same process, however the link between release probability and morphologically docked synaptic vesicles is poorly understood. Therefore, I used state-of-theart cryopreservation of unstimulated hippocampal slice cultures from wild-type mice to probe whether Schaffer collateral and mossy fiber synapses organize vesicle pools in ways that shape the functional properties at each synapse, i.e. short-term plasticity, RRP, and release probability.

2. Mossy fiber synapses contain a population of large, clear-core vesicles with diameters greater than $60 \mathrm{~nm}$ (giant vesicles) whose purpose, molecular identity, and function are poorly-understood (Henze et al., 2002b; Laatsch and Cowan, 1966). These are postulated to be the morphological correlates of giant monoquantal events in rodent acute slices observed in CA3 pyramidal neurons and are of mossy fiber origin (Henze et al., 1997, 2002b). I explore changes in giant vesicle populations in ex vivo and in vitro preparations, after pharmacological blockade of network activity, and in mossy fiber synapses lacking Munc13 priming proteins to determine if giant vesicles are activity-dependent or an artifact of slice cultures. Further, I explore whether giant monoquantal events occur in mossy fiber synapses from hippocampal slice culture and whether the morphological and functional observations are correlated.

3. Mossy fiber synapses contain a number of DCVs and secrete a variety of neuropeptides (Commons and Milner, 1995; Danzer and McNamara, 2004; Dieni et al., 2015; Henze et al., 2000; McQuiston and Colmers, 1996; Rollenhagen et al., 2007; Salin et al., 1995), however the exact location of DCV exocytosis and whether DCVs fuse under basal conditions in mossy fiber boutons are unknown. I examine changes in the spatial distribution of DCVs in mossy fiber synapses at rest from ex vivo and in vitro tissue preparations. Furthermore, I explore the effects of pharmacological manipulation of presynaptic cAMP levels on the spatial distribution of DCVs in mossy fiber boutons since neuropeptide secretion in mossy fibers is implicated in mossy fiber synaptic plasticity (Li et al., 2010; Nakamura et al., 2007; Salin et al., 1995). 


\section{Materials and Methods}

The following methods section was written by me and has been published in Cell Reports (Maus et al., 2020).

\subsection{Hippocampal slice cultures:}

Table 3. Reagents and equipment for hippocampal organotypic slice cultures.

\begin{tabular}{|lll|}
\hline $\begin{array}{l}\text { Reagents for hippocampal slice } \\
\text { culture }\end{array}$ & Source & Catalog Number \\
\hline Basal Medium Eagle (BME) & Thermo Fisher Scientific & Cat No. 41010026 \\
\hline $\begin{array}{l}\text { GlutaMAX Supplement } \\
\text { Hank's Balanced Salt Solution, } \\
\text { Ca }^{2+}, \text { Mg }^{2+} \text { (HBSS) }\end{array}$ & Thermo Fisher Scientific & Cat. No. 35050038 \\
\hline $\begin{array}{l}\text { Horse Serum, heat inactivated } \\
\text { Kynurenic acid }\end{array}$ & Thermo Fisher Scientific & Cat No. 24020091 \\
\hline Millicell cell culture inserts & Sigma-Aldrich & Cat No. 26050088 \\
\hline $\begin{array}{l}\text { Millipore membrane confetti } \\
\text { Minimum Essential Medium } \\
\text { (MEM) }\end{array}$ & Merck Millipore & Cat. No. K3375 \\
\hline Equipment & Thermo Fisher Scientific & Cat. No. PICM03050 \\
\hline Mcllwain tissue chopper & Company & Cat. No. FHLC04700 \\
\hline
\end{tabular}

\subsubsection{Slice cultures made from $C 57 B L / 6 N$ wild-type mice}

Mouse pups at postnatal day (P)3-7 were decapitated and the brain was removed and placed quickly into ice-cold preparation medium (97 mL Hank's balanced salt solution, $2.5 \mathrm{~mL} 20 \%$ glucose, and $1 \mathrm{~mL} 100 \mathrm{mM}$ kynurenic acid, pH adjusted to 7.4). Both hippocampi were dissected with excess EC attached and transferred to a tissue chopper (Mcllwain tissue chopper) platform where they were sectioned into $300 \mu \mathrm{m}$-thick slices perpendicular to the long-axis of the hippocampus. Slices were washed off the stage into ice-cold preparation medium. Slices were selected based on cell lamination and slice morphology and transferred onto sterile Millipore membrane confetti pieces that were placed on top of 6-well membrane inserts in pre-equilibrated culture medium $(22.44 \mathrm{~mL}$ ddH2O, $25 \mathrm{~mL} 2 \times \mathrm{MEM}, 25 \mathrm{~mL} B M E$, $1 \mathrm{~mL}$ GlutaMAX, $1.56 \mathrm{~mL} 40 \%$ Glucose, $25 \mathrm{~mL}$ horse serum). Residual medium around the slices was aspirated using a P200 pipette. A maximum of 12 hippocampal slices were cultured 
per animal and a maximum of four slices plated per membrane insert. Slices were maintained for 14 and 28 days at $37^{\circ} \mathrm{C}$ and $5 \% \mathrm{CO}_{2}$ with a medium change every 2-3 days.

\subsubsection{Slice cultures made from Munc13-deficient and control mice}

For the generation of Munc13-1 (Unc13A) and Munc13-2 (Unc13B) double knock-out (DKO) (Augustin et al., 1999; Varoqueaux et al., 2002) and control (CTRL) littermates mice with Unc13A $^{+/-}$(Munc13-1) Unc13B ${ }^{+/-}$(Munc13-2) genotype were bred with Unc13A ${ }^{+/-}$Unc13B $^{-/}$.

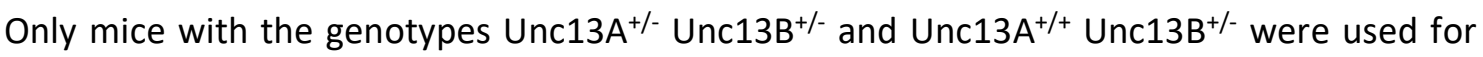
tomographic analysis of CTRL animals. Slice cultures from Munc13-1/2 DKO and CTRL littermates were prepared at embryonic day (E)18 due to the severe perinatal lethal phenotype of the Munc13-1/2 DKO (Varoqueaux et al., 2002). The slice culture procedure was identical to that of wild-type mice outlined in the previous section. However, due to the earlier developmental age of the pups, the hippocampi were smaller and therefore a maximum of 8 hippocampal slices were cultured per animal. Slices were maintained for 28 days at $37^{\circ} \mathrm{C}$ and $5 \% \mathrm{CO}_{2}$ with a medium change every 2-3 days.

\subsection{High-pressure freezing, automated freeze substitution, and sample preparation for electron microscopy}

\subsubsection{High-pressure freezing of organotypic slice cultures}

Table 4. Reagents and equipment for high-pressure freezing.

\begin{tabular}{|l|ll|}
\hline $\begin{array}{l}\text { Reagents used for high-pressure } \\
\text { freezing }\end{array}$ & Source & Catalog number \\
\hline 1-Hexadendene & Sigma Aldrich & Cat. No. 52276-5mL \\
\hline Bovine Serum Albumin & Biomol & Cat. No. 01400.1 \\
\hline Bovine Serum Albumin & Sigma Aldrich & Cat. No. A2153 \\
\hline Equipment & Company & Source \\
\hline High-pressure freezing device & Leica & Wetzlar, Germany \\
\hline
\end{tabular}

Slices were transferred to fresh culture medium 24 hours prior to fixation. Slices were transferred to pre-equilibrated slice culture medium and excess membrane was carefully removed with a razor blade. Slices were briefly submerged in slice culture medium containing $20 \%$ bovine serum albumin (BSA), which acts as a non-penetrating cryoprotectant, and loaded into the $100 \mu \mathrm{m}$-deep cavity of an aluminum planchette (type A, Leica Cat\# 16770126, outer diameter $6 \mathrm{~mm}$, inner cavity depth $100 \mu \mathrm{m})$. The planchette was then transferred to the 
middle plate on the HPF device (Leica HPM100 LS) and covered with the flat side of a type B aluminum planchette (Leica Cat\# 16770127) coated with 1-Hexadecene (Sigma Aldrich), which facilitates separation of planchettes at post-cryofixation steps. Since gas is compressible, and the freezing process occurs at $\sim 2000$ bar atmospheric pressure, care was taken not to introduce air bubbles into the planchette cavity at any stage of the process. After HPF, cryofixed samples were stored in liquid nitrogen until further processing.

\subsubsection{Acute brain slice preparation}

Wild-type animals at P18 were anaesthetized, quickly decapitated, and brains were removed from the skull. Hippocampi were dissected from the cortices, placed on a tissue chopper, and $200 \mu \mathrm{m}$-thick sections were cut. Slices were removed from the tissue chopper and placed in HEPES-buffered artificial cerebrospinal fluid (ACSF) containing 20\% BSA as a non-penetrating cryoprotectant. The CA3 and CA1 were isolated from the acute slice with a biopsy punch (diameter of $1.5 \mathrm{~mm}$ ) and placed in the $200 \mu \mathrm{m}$-deep cavity of a $3 \mathrm{~mm}$ aluminum planchette (Leica Cat\# 1677141 for type A). Hexadecene-coated lids (the flat side of type B $3 \mathrm{~mm}$ aluminum planchettes; Leica Cat\# 1677142) were placed over the sample and quickly highpressure frozen. The time between decapitation and HPF was no longer than 5 minutes.

\subsubsection{Transcardial perfusion}

Table 5. Reagents and equipment for chemical fixation.

\begin{tabular}{|l|l|l|}
\hline Reagents for chemical fixation & Source & Catalog number \\
\hline $\begin{array}{l}\text { 25\% Glutaraldehyde } \\
\text { Di-Sodium hydrogen phosphate } \\
\text { dihydrate }\end{array}$ & Electron microscopy sciences & Cat. No. 16220 \\
\hline Paraformaldehyde & Merck & Cat. No. 1.06580.1000 \\
\hline $\begin{array}{l}\text { Sodium dihydrogen phosphate } \\
\text { monohydrate }\end{array}$ & Serva & Cat. No. 31628.02 \\
\hline Sodium cacodylate trihydrate & Merck & Cat. No. 1.06346.0500 \\
\hline Equipment & Company Aldrich & Cat. No. C0250-100G \\
\hline Leica Vibratome & Leica & Wetzlar, Germany \\
\hline
\end{tabular}

P28 wild-type mice were given an intraperitoneal injection of a lethal dose of Avertin (2,2,2,Tribromoethanol). Once the mice were deeply anaesthetized, they were transcardially perfused first with $0.9 \%$ sodium chloride followed by one of two fixatives: Perfusion Fixative 1 (PF1): ice-cold 4\% PFA, 2.5\% GA in $0.1 \mathrm{M}$ phosphate buffer (PB), pH 7.4 (Rollenhagen et al., 


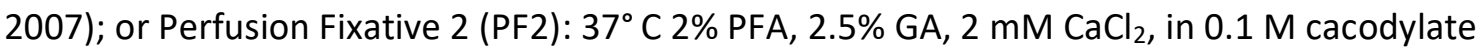
buffer (Chicurel and Harris, 1992). The brains were dissected from the mice and post-fixed with their respective fixative overnight at $4^{\circ} \mathrm{C}$ with gentile agitation. The brains were washed thoroughly with ice-cold $0.1 \mathrm{M} \mathrm{PB}(\mathrm{pH} 7.4)$ before $100 \mu \mathrm{m}$-thick sections were cut using a vibratome (Leica VT1200S; amplitude of $1.5 \mathrm{~mm}$, cutting speed $0.1 \mathrm{~mm} / \mathrm{sec}$ ). Sections were briefly stored in $0.1 \mathrm{M}$ PB before HPF. The CA3 and CA1 regions were excised from the sections using a biopsy punch (diameter of $1.5 \mathrm{~mm}$ ) and high-pressure frozen in $3 \mathrm{~mm}$ aluminum planchettes. Tissue was frozen in a mixture of $20 \%$ BSA dissolved in 0.1 M PB.

\subsubsection{Immersion fixation of hippocampal slice cultures}

Hippocampal slice cultures at days in vitro (DIV) 28 were quickly removed from the cell culture incubator and immersed in one of two fixatives: Immersion Fixative 1 (IF1): ice-cold 4\% PFA, 2.5\% GA in $0.1 \mathrm{M} \mathrm{PB}, \mathrm{pH} 7.4$ (Rollenhagen et al., 2007); or Immersion Fixative 2 (IF2): $37^{\circ} \mathrm{C} 2 \%$ PFA, 2.5\% GA, $2 \mathrm{mM} \mathrm{CaCl}_{2}$, in $0.1 \mathrm{M}$ cacodylate buffer (Chicurel and Harris, 1992). Slices immersed in IF1 were incubated overnight at $4^{\circ} \mathrm{C}$ with gentile agitation. Slices were immersed in IF2 at an initial temperature of $37^{\circ} \mathrm{C}$ and were slowly cooled to room temperature for one hour with gentile agitation and then at $4^{\circ} \mathrm{C}$ overnight. The slices were then washed thoroughly with $0.1 \mathrm{M} \mathrm{PB}(\mathrm{pH}$ 7.4). The CA3 region was isolated from the fixed slice with a biopsy punch (1.5 mm in diameter) and cryofixed in 20\% BSA dissolved in $0.1 \mathrm{M} \mathrm{PB}$ (pH 7.4) as a non-penetrating cryoprotectant. Untreated hippocampal slices from the same cultures were cryo-fixed in tandem at DIV29 to serve as controls (processed as described above). 


\subsubsection{Acute pharmacological silencing experiments}

Table 6. Reagents used for pharmacological treatment of organotypic slices.

\begin{tabular}{|l|l|l|}
\hline Pharmacological agents & Source & Catalog number \\
\hline (-)-Bicuculline methochloride & Tocris Bioscience & Cat. No. 0131 \\
\hline Biocytin hydrochloride & Sigma-Aldrich & Cat. No. B1758 \\
\hline D-AP5 & Tocris Bioscience & Cat. No. 0106 \\
\hline $\begin{array}{l}\text { DCG-IV: (2S,1'R,2'R,3'R)-2-(2,3- } \\
\text { dicarboxycyclopropyl) glycine }\end{array}$ & Tocris Bioscience & Cat. No. 0975 \\
\hline Forskolin & Sigma Aldrich & Cat. No. F3917-25mg \\
\hline NBQX disodium salt & Tocris Bioscience & Cat. No. 1044 \\
\hline Tetrodotoxin & Tocris Bioscience & Cat. No. 1078 \\
\hline Tetrodotoxin citrate & Tocris Bioscience & Cat. No. 1069 \\
\hline
\end{tabular}

The protocol for the application of pharmacological agents to cultured slices was based on a previously published protocol for the application of drugs to organotypic slice cultures (Studer et al., 2014). Wild-type organotypic slices at DIV14 were placed onto new, sterile membrane inserts in a 6-well plate containing fresh, pre-equilibrated organotypic slice culture medium supplemented with one of two drug cocktails: (1) T/N/A, comprising $1 \mu \mathrm{M}$ tetrodotoxin (TTX) to block sodium propagated action potentials, $2 \mu \mathrm{M}$ 2,3-Dioxo-6-nitro-1,2,3,4tetrahydrobenzo[f]quinoxaline-7-sulfonamide (NBQX) to block excitatory postsynaptic $\alpha$ amino-3-hydroxy-5-methyl-4-isoxazolepropionic acid (AMPA) receptors, and $50 \mu \mathrm{M}$ D-(-)-2Amino-5-phosphonopentanoic acid (D-AP5) to block excitatory postsynaptic N-methyl-Daspartate (NMDA) receptors; and (2) T/D, comprising $1 \mu \mathrm{M}$ TTX and $2 \mu \mathrm{M}$ DCG-IV, an mGluR2 receptor agonist that specifically reduced mossy fiber synaptic transmission (Kamiya et al., 1996). The vehicle control (VC) used as a negative control condition comprised medium alone. Then, $50 \mu \mathrm{L}$ of medium containing either T/N/A, T/D, or VC were pipetted onto slices and incubated at $37^{\circ} \mathrm{C}$ and $5 \% \mathrm{CO}_{2}$ for 10 minutes. Slices were then prepared for HPF as described above, with the exception that the cryoprotectant-supplemented medium used prior to freezing also contained the respective drug cocktails at the indicated concentrations. 


\subsubsection{Pharmacological manipulation of presynaptic cAMP}

Wild-type organotypic slices at DIV28 were transferred onto a new membrane insert in a sixwell plate containing pre-equilibrated slice culture medium supplemented with one of two drug cocktails: (1) T/D, comprising $1 \mu \mathrm{M}$ TTX, $2 \mu \mathrm{M}$ DCG-IV and $0.08 \%$ dimethyl sulfoxide (DMSO); or (2) T/F, comprising $1 \mu \mathrm{M} \mathrm{TTX}, 0.2 \%$ additional $\mathrm{dd}_{2} \mathrm{O}$, and $25 \mu \mathrm{M}$ forskolin, an activator of AC1 that causes the enhancement of mossy fiber synaptic transmission (LópezGarcía et al., 1996; Villacres et al., 1998; Weisskopf et al., 1994). The VC used as a negative control condition comprised $1 \mu \mathrm{M}$ TTX, 0.08\% DMSO, and $0.2 \%$ additional distilled water. Slices were incubated for 15 minutes at $37^{\circ} \mathrm{C}$ and $5 \% \mathrm{CO}_{2}$ and prepared for HPF as described above, with the exception that the cryoprotectant supplemented medium used prior to freezing also contained the respective drug cocktails at the indicated concentrations.

\subsubsection{Automated freeze substitution}

Frozen slices were processed for AFS according to published protocols (Imig and Cooper, 2017; Rostaing et al., 2006). Vitrified slices were removed from liquid nitrogen storage and made accessible to freeze substitution media by separating type $A$ and type $B$ aluminum planchettes with custom-designed cryo-forceps. Samples were submersed in liquid nitrogen during this process to prevent the crystallization of water molecules in the tissue. Type $A$ planchettes containing vitrified slices were then placed in AFS buckets in EM-grade acetone (Electron Microscopy Services, Cat\# 10015) at $-90^{\circ} \mathrm{C}$. Samples were then incubated for 99 hours in 0.1\% tannic acid (Sigma Aldrich, Cat\# 403040-100G) dissolved in EM-grade acetone at $-90^{\circ}$. The samples were then fixed with $2 \% \mathrm{OsO}_{4}$ in acetone starting at $-90^{\circ} \mathrm{C}$ and slowly ramping the temperature up to $4^{\circ} \mathrm{C}$ at a rate of $5^{\circ} \mathrm{C}$ per hour until the temperature reached $-20^{\circ} \mathrm{C}$ ( 16 hours) and then at a rate of $10^{\circ} \mathrm{C}$ per hour ( 2 hours). Residual $\mathrm{OsO}_{4}$ was thoroughly washed from the samples with pre-cooled EM-grade acetone before the samples were brought to room temperature for Epon epoxy resin infiltration.

\subsubsection{Plastic embedding}

For epoxy resin embedding, samples were incubated in Eppendorf capsules in increasing concentrations of Epon (21.4 g glycidether, Serva; $14.4 \mathrm{~g}$, dodecenylsuccinic acid anhydride, DDSA, Serva; 11.3 g methylnadic anhydride, MNA, Serva; $840 \mu \mathrm{L}$, tris(dimethylaminomethyl) 
phenol, DMP-30, Electron microscopy services) resin dissolved in EM-grade acetone: 50\% Epon (4-6 hours); 90\% Epon (overnight). Samples were then transferred to fresh Eppendorf capsules and incubated in three exchanges of 100\% Epon over two days. For polymerization steps, carrier planchettes containing the osmified slices were placed sample-side up on a parafilm-covered glass slide. An Epon-filled gelatin capsule (size 00; Plano; Cat\# G29218) containing a small specimen label was inverted over the sample and polymerized by baking at $60^{\circ} \mathrm{C}$ for $24-36$ hours. Polymerized blocks were trimmed using a diamond-tipped milling device (Leica Reichert Jung Ultratrim) and planchettes were carefully removed with a razor blade to expose the tissue for subsequent ultramicrotomy.

\subsubsection{U/tramicrotomy and contrasting}

An Ultracut UCT ultramicrotome (Leica) equipped with diamond knives (Diatome, jumbo and ultra $45^{\circ}$ ) was used to acquire plastic-embedded tissue sections at three different thicknesses: $60 \mathrm{~nm}$ ultrathin sections were collected on for 2D ultrastructural analyses; $200 \mathrm{~nm}$ sections for 3D electron tomography; and $500 \mathrm{~nm}$ sections for low-magnification orientation using light microscopy. For ultrastructural analyses, sections were collected on formvar-coated grids (Electron Microscopy Services; 100 square copper; Cat\# G2100C) and stored in grid boxes until further use. For 2D ultrastructural analysis, lipid bilayer contrast was enhanced by floating $60 \mathrm{~nm}$-thick grid-mounted sections on solutions of $1 \%$ aqueous uranyl acetate for 30 minutes followed by $0.3 \%$ Reynold's lead citrate for 2 minutes. For 3D electron tomography, gold fiduciary markers were deposited on both surfaces of $200 \mathrm{~nm}$-thick grid-mounted sections with $10 \mathrm{~nm}$ gold-conjugated Protein A (Cell Microscopy Center, Utrecht, The Netherlands). To obtain light microscopic overviews of sectioned tissue, $500 \mathrm{~nm}$-thick sections were dried on glass slides, and then contrasted with methylene blue Nissl stain to visualize patterns of cell body lamination. 


\subsection{Electron microscopy}

Table 7. Reagents and equipment for sample processing and preparation for electron

\begin{tabular}{|lll|}
\hline Reagents & Source & Catalog number \\
\hline $\begin{array}{l}\text { 2,4,6- } \\
\text { Tris(dimethylaminomethyl)phenol } \\
\text { (DMP-30) }\end{array}$ & Electron microscopy sciences & Cat. No. 13600 \\
\hline $\begin{array}{l}\text { 2-Dodecenylsuccinic acid } \\
\text { anhydride (DDSA) }\end{array}$ & Serva & Cat. No. 20755.02 \\
\hline Acetone & Electron microscopy sciences & Cat. No. 10015 \\
\hline Glycidether 100 & Serva & Cat. No. 21045.02 \\
\hline Lead (II) Nitrate & Merck & Cat. No. 1.07398 .0100 \\
\hline Methylnadic anhydride (MNA) & Serva & Cat. No. 29452.02 \\
\hline Osmium tetroxide & Electron microscopy sciences & Cat. No. 19132 \\
\hline Protein A (ProtA) coupled to 10 nm & Cell Microscopy Core Products, & \\
\hline gold particles & $\begin{array}{l}\text { University Medical Center } \\
\text { Utrecht, The Netherlands }\end{array}$ & \\
\hline Sodium Citrate & Calbiochem & Cat. No. 567446 \\
\hline Tannic Acid 0.1\% & Sigma Aldrich & Cat. No. 403040-100G \\
\hline Uranyl Acetate & SPI Supplies & Cat. No. 2624 \\
\hline Equipment & Company & Source \\
\hline Leica Vibratome & Leica & Wetzlar, Germany \\
\hline EM AFS2 & Leica & Wetzlar, Germany \\
\hline Leica Reichert Jung Ultratrim & Leica & Wetzlar, Germany \\
\hline
\end{tabular}

\subsubsection{Transmission electron microscopy imaging and analysis}

For 2D ultrastructural analyses, images were acquired on an $80 \mathrm{kV}$ Leo912 TEM (Zeiss) equipped with a sharp:eye CCD camera (Tröndle, TRS) and iTEM (Olympus Soft Imaging Solutions $\mathrm{GmbH}$ ) software. Schaffer collateral and mossy fiber synapses were identified according to their distinct morphologies in montaged images acquired at 5,000x magnification (image pixel size $=2.269 \mathrm{~nm}$ ) from CA1 stratum radiatum and CA3 stratum lucidum, respectively. The CA1, CA3b and CA3c regions containing Schaffer collateral and mossy fiber synapses, respectively, were acquired at 20,000x magnification (image pixel size $=0.592 \mathrm{~nm}$ ). Material exhibiting signs of freezing damage, i.e. ice crystal formation, were identified according to published qualitative criteria (Möbius et al., 2010) and excluded from further analysis.

In forskolin-treated and corresponding VC slices (see Pharmacological manipulation of presynaptic $C A M P$ ), the following morphological parameters were quantified from 2D 
electron micrographs using the IMOD package (Kremer et al., 1996) in combination with the imodinfo and $m t$ programs: (i) presynaptic bouton area and membrane circumference, (ii) active zone number and length, (iii) spine area and presynaptic contact length, and (iv) mitochondrial number, area, and circumference.

\subsubsection{Electron tomography and data analysis}

Table 8. Imaging software and equipment for 2D electron microscopy and 3D electron tomography.

\begin{tabular}{|l|l|l|}
\hline Imaging and Analysis Software & Supplier & Source \\
\hline IMOD software & Kremer et al., 1996 & https://bio3d.colorado.edu/imod/ \\
\hline iTEM software & Emsis GMBH & Emsis GMBH \\
\hline SerialEM software & $\begin{array}{l}\text { University of Colorado, Boulder, } \\
\text { Colorado, US }\end{array}$ & $\begin{array}{l}\text { http://bio3d. } \\
\text { colorado.edu/SerialEM/ }\end{array}$ \\
\hline Equipment & Company & Source \\
\hline $\begin{array}{l}\text { Leo912 Transmission electron } \\
\text { microscope }\end{array}$ & Zeiss & Jena, Germany \\
\hline $\begin{array}{l}\text { JEM 2100 transmission electron } \\
\text { microscope }\end{array}$ & Jeol & Tokyo, Japan \\
\hline
\end{tabular}

Electron tomograms from Schaffer collateral and mossy fiber active zone release sites were generated on a $200 \mathrm{kV}$ JEM2100 TEM (JEOL) equipped with an Orius SC1000 digital camera (Gatan). Single-axis tilt series $\left(-60^{\circ}\right.$ to $+60^{\circ}, 1^{\circ}$ increments) were acquired at $30,000 \mathrm{x}$ magnification with a $2 x$ binning factor (image pixel size $=0.52 \mathrm{~nm}$ ) using Serial EM software (Mastronarde, 2005). Only synapses in which the synaptic cleft was clearly visible at $0^{\circ}$ tilt were selected for reconstruction using the weighted back-projection feature of the IMOD package (Kremer et al., 1996) and a 3x binning factor (tomogram voxel dimensions $x, y, z=$ $1.554 \mathrm{~nm})$. The location and dimensions of reconstructed synaptic active zones were identified according to four morphological criteria: 1) an accumulation of presynaptic vesicles, 2) a directly apposing postsynaptic density, 3) a widening of the synaptic cleft, and 4) the presence of electron dense trans-synaptic cleft material (Gray, 1959; High et al., 2015; Palay, 1956). These criteria were necessary in some cases for analysis of mossy fiber synapses due to the presence of multiple active zones in proximity to one another and that additional protein contrasts were not used for sections imaged with electron tomography. Vesicles within $100 \mathrm{~nm}$ of the active zone were segmented manually as size-matched spheres positioned at the vesicular midline, i.e. the tomographic slice of largest vesicular diameter. 
The diameter of segmented spheres was adjusted to correspond to the outer leaflet of the vesicle lipid bilayer. Non-spherical organelles (e.g. endoplasmic reticulum, tubular endosomal intermediates) were occasionally observed in tomographic reconstructions, but excluded from the analysis. Active zones were segmented as open contours corresponding to the inner leaflet of the presynaptic plasma membrane.

Vesicle radii and active zone surface areas were extracted from segmented tomograms using the imodinfo program of the IMOD package (Kremer et al., 1996). The closest approach of vesicles to the active zone was measured in Euclidean space using the $m t k$ program of the IMOD package (Kremer et al., 1996). Docked synaptic vesicles in direct contact with the active zone membrane were manually quantified according to the criterion that no measurable distance was observed between the outer leaflet of the vesicle lipid bilayer and the inner leaflet of the presynaptic membrane (i.e. when the dark pixels corresponding to the vesicular membrane were contiguous with those of the plasma membrane). The number of vesicles measured within discrete distances from the active zone membrane [i.e. 0-2 (docked), 0-40, and $0-100$ ] were normalized to the active zone area and reported as a spatial density (i.e. number of vesicles per $0.01 \mu^{2}$ active zone). Vesicles were classified into three morphological categories according to their diameter and lumenal content: clear-cored vesicles with a diameter less than $60 \mathrm{~nm}$ were classified as synaptic vesicles; clear-cored vesicles with a diameter exceeding $60 \mathrm{~nm}$ were classified as giant vesicles; and vesicles with a prominent electron-dense core were classified as DCVs irrespective of their diameter.

\subsection{RRP calculations}

In mossy fiber-CA3 spine synapses, my calculations of mean docked vesicle numbers per active zone and per mossy fiber bouton were based on the mean number of docked vesicles per unit of active zone area (0.97 synaptic vesicles, 0.1 giant vesicles and 0.05 DCVs per $0.01 \mu \mathrm{m}^{2}$ active zone area) quantified in this work, as well as previously published estimates of the mean active zone surface area $\left(0.12 \mu \mathrm{m}^{2}\right)$ and mean active zone number (29.75 active zones) per bouton in P28 rat mossy fiber boutons (Rollenhagen et al., 2007). I calculated the mean docked vesicle numbers per active zone (11.6 synaptic vesicles, 1.1 giant vesicles, 0.6 DCV) and per bouton ( 345 synaptic vesicles, 33 giant vesicles, 18 DCVs) in mossy fiber synapses from DIV28 slice cultures as well as the mean number of total membrane-proximal 
(within 0-40 $\mathrm{nm}$ of the active zone; 25.1 synaptic vesicles, 3 giant vesicles, $2.3 \mathrm{DCVs}$ ) vesicles per active zone.

I extrapolated the surface area and volume of all docked vesicles according to their size and morphological classification (mean diameters: synaptic vesicles, $45.17 \mathrm{~nm}$; giant vesicles, $85.77 \mathrm{~nm}$; DCVs $74.41 \mathrm{~nm}$ ). Based on a specific membrane capacitance of $1 \mu \mathrm{F} / \mathrm{cm}^{2}$ (Hallermann et al., 2003), I estimated that, (i) the fusion of all docked vesicles, irrespective of their type, would correspond to a membrane capacitance increase of $~ 33 \mathrm{fF}$ per mossy fiber bouton, and (ii) that the fusion of all vesicles within 0-40 $\mathrm{nm}$ of the active zone membrane would correspond to a membrane capacitance increase of $~ 80 \mathrm{fF}$ per mossy fiber bouton. A limitation of this approach is that my data, which is based exclusively on tomograms from mossy fiber-CA3 spine synapses, does not take into account synapses made via filopodial extensions (Acsády et al., 1998).

\subsection{Electrophysiology}

Table 9. Software used for electrophysiology acquisition and analysis.

\begin{tabular}{|lll|}
\hline Software & Supplier & Source \\
\hline $\begin{array}{l}\text { GraphPad Prism 5 } \\
\text { and 7 }\end{array}$ & $\begin{array}{l}\text { GraphPad } \\
\text { Software }\end{array}$ & http://www.graphpad.com \\
\hline Axograph X 1.3.3 & John Clements & https://axograph.com \\
\hline Patchmaster v2X80 & $\begin{array}{l}\text { HEKA/Harvard } \\
\text { Bioscience }\end{array}$ & http://www.heka.com/products/products main.html\#soft_pm \\
\hline
\end{tabular}

\subsubsection{Miniature excitatory postsynaptic currents in CA3 pyramidal neurons in slice culture at DIV14}

\section{Performed by Dr. Bekir Altas}

All recordings of miniature EPSCs (mEPSCs) from CA3 pyramidal cells were performed in wildtype organotypic slice cultures at DIV14. Prior to recording, slices were incubated for 30 minutes in an interface chamber with carbogen-saturated ACSF $(120 \mathrm{mM} \mathrm{NaCl}, 26 \mathrm{mM}$ $\mathrm{NaHCO}_{3}, 10 \mathrm{mM}$-glucose, $2 \mathrm{mM} \mathrm{KCl}, 2 \mathrm{mM} \mathrm{MgCl}$, and $2 \mathrm{mM} \mathrm{CaCl}_{2}$, and $1 \mathrm{mM} \mathrm{KH}_{2} \mathrm{PO}_{4}$ with an osmolarity of $304 \mathrm{mOsm})$. One or two CA3 pyramidal cells were then whole-cell voltage clamped using a glass pipette (2.5-3.0 M $\Omega$ ) filled with internal solution $(100 \mathrm{mM} \mathrm{KCl}, 50 \mathrm{mM}$ K-gluconate, 10 mM HEPES, 4 mM ATP-Mg, 0.3 mM GTP-Na, 0.1 mM EGTA, and 0.4\% biocytin, 
$\mathrm{pH} 7.4$ with an osmolarity of $300 \mathrm{mOsm}$ ) and the holding potential was set at $-70 \mathrm{mV}$ using an EPC-10 amplifier [Patchmaster 2 software (HEKA/Harvard Bioscience)]. For measurements of mEPSC amplitudes and frequencies, slices were initially perfused with $1 \mu \mathrm{M}$ TTX and $10 \mu \mathrm{M}$ bicuculline and mEPSCs were then recorded for 10 minutes, after which the slices were perfused with $1 \mu \mathrm{M}$ TTX, $10 \mu \mathrm{M}$ bicuculline, and $2 \mu \mathrm{M}$ DCG-IV for 15 minutes to record DCGIV insensitive mEPSCs. Measurements of all mEPSCS (TTX/bicuculline) were recorded in two5-minute epochs, while measurements of non-mossy fiber mEPSCs (TTX/bicuculline/DCG-IV) were recorded in three-5 minunte epochs. The last epochs of each recording were used for mEPSC analysis. All electrophysiological traces were analyzed using Axograph $\mathrm{X}$ software (AxoGraph Scientific) using a template fit algorithm for automatic event detection (Jonas et al., 1993; Pernía-Andrade et al., 2012). After recordings, slices were fixed and biocytin-filled CA3 pyramidal cell were stained with Alexa Fluoro-555-labeled streptavidin (see Light Microscopic Analysis section for detailed procedure).

Only cells exhibiting a reduction in the mEPSC frequency upon application of DCG-IV were analyzed (reduction range 16.9-88.6\%; mean 59.2\%). The threshold for mEPSC detection was set to $8 \mathrm{pA}$. The amplitude distribution of mEPSC events was plotted ( $1 \mathrm{pA}$ bins) for epochs prior to and following the application of DCG-IV. The remaining DCG-IV-insensitive events were subtracted from the events recorded prior to drug application in the respective bins to isolate the DCG-IV-sensitive component (likely of mossy fiber origin). Operating on the assumption that DCG-IV-sensitive mEPSC events result from the fusion of docked vesicles at mossy fiber active zone release sites, the statistical mode of mEPSC amplitudes (10 pA) was correlated with the statistical mode of docked vesicle diameters ( $44 \mathrm{~nm}$ ). Since vesicle diameters were measured between the outer leaflets of the vesicle lipid bilayer, lumenal volumes were calculated by subtracting the thickness of the lipid bilayers (each approximately $4 \mathrm{~nm}$-thick as measured from center-to-center of inner and outer leaflets). Based on a previous, conceptually analogous study (Bruns et al., 2000), I assumed uniform neurotransmitter loading irrespective of vesicle size and negligible saturation of postsynaptic receptors to estimate that an $\mathrm{mEPSC}$ amplitude of approximately $30 \mathrm{pA}$ would arise from the fusion of a vesicle with a diameter of $60 \mathrm{~nm}$ (size threshold for classification of a giant vesicle). 


\subsubsection{Release probability and short-term plasticity of Schaffer collateral and mossy fiber synapses in slice cultures at DIV14 and DIV28}

\section{Performed by Dr. Chungku Lee}

Whole-cell voltage-clamp recordings of evoked EPSCs in CA1 and CA3 neurons in wild-type organotypic slice cultures at DIV14 and DIV28 were performed by extracellular stimulation of Schaffer collaterals and mossy fibers, respectively. Short current pulses (0.1 ms, 100-1000 $\mu \mathrm{A})$ were applied using an ACSF-filled bipolar theta glass electrode (Science Products, Hofheim, Germany). Whole-cell recordings were made at $-70 \mathrm{mV}$ in the presence of $100 \mu \mathrm{M}$ picrotoxin to block inhibitory currents. Borosilicate glass pipettes (4-5 M 2 ; P.Clamp Glass \#0010, WPI) were filled with intracellular solution ( $115 \mathrm{mM}$ Cs-methanesulfonate, $10 \mathrm{mM}$ HEPES, $10 \mathrm{mM}$ EGTA, $5 \mathrm{mM} \mathrm{MgCl2}, 5 \mathrm{mM}$ QX-314, $4 \mathrm{mM}$ Na2-ATP, $0.3 \mathrm{mM} \mathrm{Na2-GTP}$, and 0.2 \% biocytin, pH 7.4). The serial resistance was compensated by $45 \%-80 \%$ and only cells with serial resistances below $12 \mathrm{M} \Omega$ were analyzed. All experiments were recorded by an EPC-10 USB double amplifier and Patch-Master (Ver. 2X73.5) software (HEKA electronics) and the obtained data were analyzed using Axograph (Ver.1.5.4) software (Axograph Scientific). Statistical analysis of data was performed with GraphPad Prism (versions 5 or 7) software (GraphPad Software Inc., La Jolla, CA).

\subsection{Light microscopic analysis}

\section{Performed with help from Dr. Benjamin Cooper and Manuela Schwark}

\subsubsection{Sample preparation for confocal microscopy}

To demonstrate the correct anatomical organization of the mossy fiber pathway in organotypic slices, cultures were immersion-fixed in 4\% PFA in 0.1 M PB (pH 7.4) overnight at $4^{\circ} \mathrm{C}$. Slices were washed in 0.1 M PB ( $\mathrm{pH} 7.4$ ) and then permeabilized and blocked in $10 \%$ normal goat serum (NGS), $0.3 \%$ Triton X-100, and $0.1 \%$ cold water fish skin gelatin in $0.1 \mathrm{M} P B$ $\left(\mathrm{pH}\right.$ 7.4) overnight at $4^{\circ} \mathrm{C}$. Whole-mount immunolabelling was performed by incubating slices overnight at $4^{\circ} \mathrm{C}$ in $5 \% \mathrm{NGS}, 0.3 \%$ Triton $\mathrm{X}-100$ and $0.1 \%$ fish skin gelatin in $0.1 \mathrm{M} \mathrm{PB}$ (pH 7.4) containing primary antibodies against synaptic vesicle clusters within the synaptic terminals of mossy fiber projections [polyclonal rabbit anti-synaptoporin, Synaptic Systems (Cat\# 102 
003), 1:1000 dilution] and cell bodies and dendritic arborizations [polyclonal chicken antiMAP2, Novus Biologicals (Cat\# NB300-213), 1:600 dilution]. Slices

Table 10. Reagents and equipment used for immunostaining and light microscopy.

\begin{tabular}{|c|c|c|c|}
\hline Antibodies & Dilution & Source & Catalog number \\
\hline Chicken anti-MAP2 antibody & $1: 600$ & Novus & Cat. No. NB 300-213 \\
\hline $\begin{array}{l}\text { Goat anti-Chicken IgG } \\
\text { secondary antibody, Alexa } \\
488\end{array}$ & 1:1000 & Thermo Fisher Scientific & Cat. No. A-11039 \\
\hline $\begin{array}{l}\text { Goat anti-Rabbit IgG } \\
\text { secondary antibody, Alexa } \\
555\end{array}$ & $1: 1000$ & Thermo Fisher Scientific & Cat. No. A21429 \\
\hline $\begin{array}{l}\text { Goat anti-Rabbit IgG } \\
\text { secondary antibody, Alexa } \\
\text { Fluor } 488\end{array}$ & 1:1000 & Thermo Fisher Scientific & Cat. No. A-11008; \\
\hline $\begin{array}{l}\text { Mouse anti-bassoon } \\
\text { antibody }\end{array}$ & $1: 400$ & Enzo & $\begin{array}{l}\text { Cat. No. ADIVAM- } \\
\text { PS003-F }\end{array}$ \\
\hline $\begin{array}{l}\text { Rabbit anti-synaptoporin } \\
\text { antibody }\end{array}$ & $1: 500$ & SynapticSystems & Cat. No. 102003 \\
\hline $\begin{array}{l}\text { Goat anti-Mouse secondary } \\
\text { antibody, ATTO647N }\end{array}$ & $1: 100$ & Rockland & Cat. No. $610-156-12$ \\
\hline $\begin{array}{l}\text { Goat anti-Rabbit secondary } \\
\text { antibody, START580 }\end{array}$ & $1: 100$ & Abberior & $\begin{array}{l}\text { Cat. No. ST580-1002- } \\
\text { 500UG }\end{array}$ \\
\hline $\begin{array}{l}\text { Alexa Fluor } 555 \text { streptavidin } \\
\text { conjugate }\end{array}$ & $1: 500$ & Thermo Fisher Scientific & $\begin{array}{l}\text { Cat. No. S32355; } \\
\text { RRID: AB_2571525 }\end{array}$ \\
\hline $\begin{array}{l}\text { DAPI: 4',6-Diamidine-2'- } \\
\text { phenylindole } \\
\text { dihydrochloride }\end{array}$ & 1:1000 & Sigma-Aldrich/Roche & Cat. No. 10236276001 \\
\hline $\begin{array}{l}\text { Imaging and Analysis } \\
\text { Software }\end{array}$ & Supplier & Source & \\
\hline ImageJ & $\begin{array}{l}\text { National Institutes } \\
\text { of Health }\end{array}$ & https://imagej.nih.gov/ij & \\
\hline Leica LAS AF & $\begin{array}{l}\text { Leica } \\
\text { Microsystems }\end{array}$ & $\begin{array}{l}\text { http://www.leica- } \\
\text { microsystems.com }\end{array}$ & \\
\hline Equipment & Company & Source & \\
\hline SP5 Confocal Microscope & Leica & Wetzlar, Germany & \\
\hline Zeiss Apotome image Z.1 & Zeiss & Jena, Germany & \\
\hline
\end{tabular}

were washed in $0.1 \mathrm{M} \mathrm{PB}(\mathrm{pH} 7.4)$ and primary antibodies were visualized by a two-hour incubation at room temperature in $5 \%$ NGS, $0.1 \%$ Triton $\mathrm{X}-100$ and $0.1 \%$ fish skin gelatin in 0.1 M PB (pH 7.4) containing goat anti-rabbit Alexa 555 [Thermo Fisher (Cat\# A21429), dilution 1:1000] and goat anti-chicken Alexa 488 [Thermo Fisher (Cat\# A-11039), dilution 
1:1000]. Following final washing steps in $0.1 \mathrm{M} \mathrm{PB}(\mathrm{pH} 7.4)$, slices were floated onto Superfrost ${ }^{\mathrm{TM}}$ glass slides with the membrane confetti in contact with the slide and MenzelGläser \#1.5 glass coverslips were mounted using Aqua-Poly/Mount mounting medium (Polysciences, Inc., Cat\# 18606-20).

To resolve active zone release sites within mossy fiber boutons, slices were removed from culture inserts and fixed by overnight immersion in 4\% PFA in $0.1 \mathrm{M} \mathrm{PB}(\mathrm{pH}$ 7.4). Slices were washed in 0.1 M PB (pH 7.4) and then cryoprotected in an increasing sucrose gradient (from $10 \%$ to $30 \%)$ in $0.1 \mathrm{M} \mathrm{PB}(\mathrm{pH} 7.4)$ until saturation and then positioned slice-side down (confetti-side up) as flat as possible on the inner base of a quadratic $10 \times 10 \times 10 \mathrm{~mm}$ form made out of aluminum foil. The form was carefully filled with liquid Tissue-Tek ${ }^{\circledR}$ OCT compound (Sakura, Cat\# 4583) and then rapidly frozen on a liquid nitrogen-cooled aluminum block. Frozen slices were stored at $-80^{\circ} \mathrm{C}$ until cryosectioning. Prior to cryosectioning, blocks were pre-equilibrated in the cryostat at $-20^{\circ} \mathrm{C}$ the night before sectioning. The aluminum foil was removed and the frozen OCT block was mounted slice-side up on a specimen stub with OCT in a precooled (specimen holder, $-18^{\circ} \mathrm{C}$; chamber, $-18^{\circ} \mathrm{C}$ ) cryostat (Leica CM3050 S). Once the temperature of the embedded slice had equilibrated, unnecessary OCT compound was trimmed away with a razor blade and $10 \mu \mathrm{m}$-thick cryosections were made through the organotypic slice and thaw-mounted on Superfrost ${ }^{\mathrm{TM}}$ slides. Slides were air-dried at room temperature for 30 minutes and a hydrophobic pen (DAKO, Cat\# S2002) was used to delineate the border of the slide surface. The hydrophobic pen helped minimize the volume of antibodies used for immunostaining. Slides were washed briefly in $0.1 \mathrm{M} \mathrm{PB}(\mathrm{pH} 7.4)$ and incubated for 90 minutes at room temperature in $10 \%$ NGS, $0.3 \%$ Triton X-100, and $0.1 \%$ fish skin gelatin in $0.1 \mathrm{M} \mathrm{PB}(\mathrm{pH} 7.4)$. Slices were then incubated overnight at $4^{\circ} \mathrm{C}$ in $3 \% \mathrm{NGS}, 0.3 \%$ Triton $\mathrm{X}-100$ and $0.1 \%$ fish skin gelatin in $0.1 \mathrm{M} \mathrm{PB}(\mathrm{pH} 7.4)$ containing primary antibodies for the detection of synaptic vesicle clusters within the synaptic terminals of mossy fiber projections [polyclonal rabbit anti-synaptoporin, Synaptic Systems (Cat\# 102 003), 1:1000 dilution] and presynaptic active zones [monoclonal mouse anti-bassoon, Enzo Life Sciences (Cat\# SAP7F407), 1:400 dilution]. Slices were washed in $0.1 \mathrm{M} \mathrm{PB}(\mathrm{pH} 7.4)$ and primary antibodies were visualized by two-hour incubation at room temperature in $5 \%$ NGS, $0.1 \%$ Triton $\mathrm{X}-100$ and $0.1 \%$ fish skin gelatin in $0.1 \mathrm{M} \mathrm{PB}(\mathrm{pH} 7.4)$ containing goat anti-rabbit Alexa 488 [Thermo Fisher (Cat\# A11008), dilution 1:1000] and goat anti-mouse Alexa 555 [Thermo Fisher (Cat\# A21424), dilution 1:1000]. Following a brief wash in 0.1 M PB, slides were dipped 
in distilled water and Menzel-Gläser \#1,5 coverslips were mounted using Aqua-Poly/Mount mounting medium (Polysciences, Inc., Cat\# 18606-20).

Confocal light microscopic analysis of biocytin-filled CA3 pyramidal cells was performed to validate that electrophysiological recordings were of the correct filled cells, as assessed by the anatomical location within the hippocampal subfields and morphological features (i.e, pyramidal soma, presence of large, complex spines in the proximal regions of apical dendritic arborizations) (see Figure $5 \mathrm{D}$ ). Immediately following mEPSC recordings and removal of the patch pipette, biocytin-filled CA3 pyramidal cells (see Electrophysiology section above for detailed procedure) were fixed for light microscopic analysis by overnight immersion of the slice in 4\% PFA in 0.1 M PB (pH 7.4). Slices were washed in $0.1 \mathrm{M} \mathrm{PB}(\mathrm{pH} 7.4)$ and then incubated overnight at $4^{\circ} \mathrm{C}$ in $10 \% \mathrm{NGS}, 0.3 \%$ Triton $\mathrm{X}-100$, and $0.1 \%$ fish skin gelatin in $0.1 \mathrm{M}$ PB (pH 7.4). Biocytin-filled cells were visualized by incubation of slices for three hours at room temperature in streptavidin-Alexa 555 [1:500 dilution] in 5\% NGS, 0.1\% Triton X-100 and 0.1\% fish skin gelatin in 0.1 M PB (pH 7.4). Slices were washed in $0.1 \mathrm{M} \mathrm{PB}(\mathrm{pH} 7.4)$ and cell nuclei were stained by a 30-minute incubation in DAPI [300 nM in $0.1 \mathrm{M} \mathrm{PB}$ ]. Following final washing steps in $0.1 \mathrm{M} \mathrm{PB}(\mathrm{pH} 7.4)$, slices were floated onto Superfrost glass slides with the membrane confetti in contact with the slide and Menzel-Gläser \#1,5 glass coverslips were mounted using Aqua-Poly/Mount mounting medium (Polysciences, Inc., Cat\# 18606-20).

\subsubsection{Confocal imaging}

Confocal laser scanning micrographs were acquired with a Leica TCS-SP5 confocal microscope equipped with a tunable white light laser, a resonant scanner, hybrid GaAsP detectors, and a motorized stage. Tiled z-series were acquired with (i) a HCX PL APO 40.0x (NA=1.25) oil immersion objective to generate low magnification overviews of entire organotypic slices (pinhole = 3.0 AU, voxel size $x, y, z=0.3,0.3,2 \mu \mathrm{m}$ (Figure $5 \mathrm{~A}$ ) and reconstructions of biocytinfilled pyramidal neurons within CA3 stratum pyramidale (pinhole $=1.0 \mathrm{AU}$, voxel size $\mathrm{x}, \mathrm{y}, \mathrm{z}=$ 95, 95, $335 \mathrm{~nm}$ ) (Figure $5 \mathrm{D}$ ), or with (ii) a HCX PL APO CS 100x (NA=1.4) oil immersion objective to visualize mossy fiber terminals within CA3 stratum lucidum (pinhole $=1.0 \mathrm{AU}$, voxel size $x, y, z=89,89,130 \mathrm{~nm}$ ) and high magnification reconstructions of complex postsynaptic spines (thorny excrescences) emerging from the proximal dendrites of biocytinfilled CA3 pyramidal neurons (pinhole $=0.5 \mathrm{AU}$, voxel size $x, y, z=47,47,130 \mathrm{~nm}$ ). For illustration purposes thorny excrescences were subjected to spatial deconvolution by use of 
two ImageJ (National Institutes of Health; Bethesda, MD) plugins: point spread functions were generated using Diffraction point spread function 3D plugin and iterative deconvolution was performed with the Richardson-Lucy algorithm (DeconvolutionLab plugin; Biomedical Imaging Group, EPFL; Lausanne, Switzerland).

\subsubsection{Stimulated emission depletion microscopy}

\section{Imaging performed by Sinem Sertel}

Wild-type hippocampal slice cultures at DIV28 were treated for 15 minutes with $1 \mu \mathrm{M}$ TTX to block spontaneous network activity as a VC, or TTX with $25 \mu \mathrm{M}$ forskolin to activate AC1 and increase presynaptic cAMP (Huang et al., 1994a; Villacres et al., 1998). Slices were transferred to a new Millipore membrane insert containing the respective drug mixtures and $50 \mu \mathrm{l}$ of drug-containing medium was pipetted onto each slice. The plate was then placed in the incubator for 15 minutes, after which the slices were removed from the inserts and were immersed in 4\% PFA in $0.1 \mathrm{M} \mathrm{PB}\left(\mathrm{pH} \mathrm{7.4)}\right.$ for one hour at $4^{\circ} \mathrm{C}$. Slices were prepared for cryosectioning as described above with some exceptions. Sections ( $10 \mu \mathrm{m}$-thick) were thawmounted on Superfrost ${ }^{\mathrm{TM}}$ slides, blocked and permeabilized for one hour at room temperature in $5 \%$ NGS, $0.3 \%$ Triton X-100 and $0.1 \%$ fish skin gelatin in $0.1 \mathrm{M} \mathrm{PB}(\mathrm{pH} 7.4)$, and were then incubated overnight at $4^{\circ} \mathrm{C}$ in $3 \%$ NGS, $0.1 \%$ Triton $\mathrm{X}-100$, and $0.1 \%$ fish skin gelatin in $0.1 \mathrm{M} \mathrm{PB}(\mathrm{pH}$ 7.4) containing primary antibodies for synaptoporin [polyclonal rabbit antisynaptoporin, Synaptic Systems (Cat\# 102 003), 1:500 dilution] and bassoon [monoclonal mouse anti-bassoon, Enzo Life Sciences (Cat\# SAP7F407), 1:400 dilution]. Sections were washed in 0.1 M PB (pH 7.4) to remove primary antibodies and were then incubated for two hours at room temperature in 3\% NGS, $0.1 \%$ Triton X-100 and 0.1\% FSG in 0.1 M PB (pH 7.4) containing goat anti-mouse ATTO647N [Rockland (Cat\# 610-156-12), dilution 1:100] and goat anti-rabbit STAR580 [Abberior (Cat\# ST580-1002-500UG), dilution 1:100]. Following a brief wash in 0.1 M PB, slides were dipped in distilled water and Menzel-Gläser \#1,5 coverslips were mounted onto them, covering the sections, using Mowiol mounting medium (Merk Millipore; Cat\# 475904).

Dual stimulated emission depletion (STED) imaging was performed on bassoon and synaptoporin immunelabelling [using an Expert Line STED (Abberior) instrument based on an IX83 inverted microscope (Olympus)]. The images were analyzed with a Matlab script 
(Mathworks) written by Sinem M. Sertel. The synaptoporin and bassoon images were thresholded to remove background and to enable the recognition of the different objects (spots). The positions of synaptoporin and bassoon objects were then calculated, and for each synaptoporin object we determined the overlap with the bassoon objects found near it (within $120 \mathrm{~nm}$ ). The total area of bassoon objects per synaptoporin object was then determined, and the mean object value per image was plotted using GraphPad Prism 7.

\subsection{Quantification and statistical analysis}

Data are represented as mean \pm standard error of the mean (SEM) unless indicated otherwise. Statistical analyses were carried out using GraphPad Prism software 7 (* when $p<0.05$; when $\mathrm{p}<0.01$, and $* * *$ when $\mathrm{p}<0.001$ ). For comparisons of two conditions (i.e. Schaffer collateral and mossy fiber synaptic profiles from DIV14 wild-type slice cultures; Figure 9 G-M) statistical differences were determined by an unpaired t-test when the data set was normally distributed as determined by a Kolmogorov-Smirnov normality test, and by a Mann-Whitney unpaired t-test if the data were not normally distributed. For pharmacological manipulation experiments, statistical significance was tested by one-way analysis of variance with Bonferroni correction as a post-test if the data set was normally distributed. If the data set was not normally distributed, Kruskal-Wallis analysis of variance test with a Dunn's comparison of all columns was performed to assess statistical significance. For the electron tomography experiments, the number of active zones analyzed for each experiment $(n)$, the number of slice cultures or animals used (N), and all EM data are summarized in Tables 15-28 and 30-31. Statistics were performed based on the number of active zones for each sample with the exception of docked vesicle diameters and unattached giant vesicles and DCVs. In the latter scenarios, the number of vesicles was used for statistical analysis and is noted in parentheses. For the 2D EM analysis of mossy fiber synaptic profiles, the number of boutons $(n)$, the number of slice cultures or animals used $(N)$, and all data are summarized in Tables 33-34. Electrophysiological analyses of mEPSC recordings were performed on 28 cells from two independent wild-type slice cultures at DIV14. Statistical difference for electrophysiological experiments measuring mEPSCs was determined by Wilcoxon matched pairs signed rank tests. Electrophysiology of Schaffer collateral and mossy fiber evoked synaptic transmission was performed on three independent slice cultures. Evoked Schaffer collateral synaptic transmission was recorded from 12 cells at DIV14 and 9 cells at DIV28. 
Evoked mossy fiber synaptic transmission was recorded from 11 cells at DIV14 and 9 cells at DIV28. For the short-term synaptic plasticity electrophysiology experiments, statistical comparisons between two groups of data were made using two-tailed unpaired Student's ttest. P-values less than 0.05 were considered significant for single and multiple comparisons, respectively. 


\section{Results}

\subsection{Differences in Schaffer collateral and mossy fiber synapse release probability are paralleled by synapse-specific differences in synaptic vesicle organization}

My primary objective was to perform a comparative 3D ultrastructural analysis of Schaffer collateral and mossy fiber synapses at rest to test the hypothesis that differences in synaptic vesicle organization at active zones contribute to their different neurotransmitter release properties, i.e. release probability and short-term plasticity. To verify that the hippocampal organotypic slices used in my experiments serve as an appropriate model system to address this aim, I performed experiments to investigate, (i) whether the anatomical development and target specificity of the mossy fiber projection remains intact during the slice culture period, and (ii) whether the functional neurotransmitter release properties of Schaffer collateral and mossy fiber synapses described in acute brain slices are also preserved in cultured slices. Having established the anatomical and functional integrity of the organotypic slice culture system, I then compared the 3D spatial organization of synaptic vesicles at active zone release sites in Schaffer collateral and mossy fiber synapses within the same slice to test the hypothesis that initial release probability at these synapses is codetermined by the availability of docked and primed vesicles.

\subsubsection{Intact target specificity of the mossy fiber pathway in hippocampal organotypic slice cultures}

To assess whether the target specificity of the mossy fiber projection from the granule cell layer to the stratum lucidum of the CA3 was preserved in our culture system, I performed whole-mount immunolabelling experiments using an anti-synaptoporin antibody to detect the synaptic vesicle channel protein synaptoporin, which is highly enriched in hippocampal granule cells (Grabs et al., 1994), and an antibody directed against microtubule-associated protein 2 (MAP-2) to visualize the cell bodies and dendrites of CA3 pyramidal cells (Figure 5 A, B) (De Camilli et al., 1984). Light microscopic analysis revealed a pattern of immunolabelling that is highly comparable to the in vivo organization of the hippocampus, in which synaptoporin puncta are restricted to the hilus, where mossy fiber collaterals form synaptic 

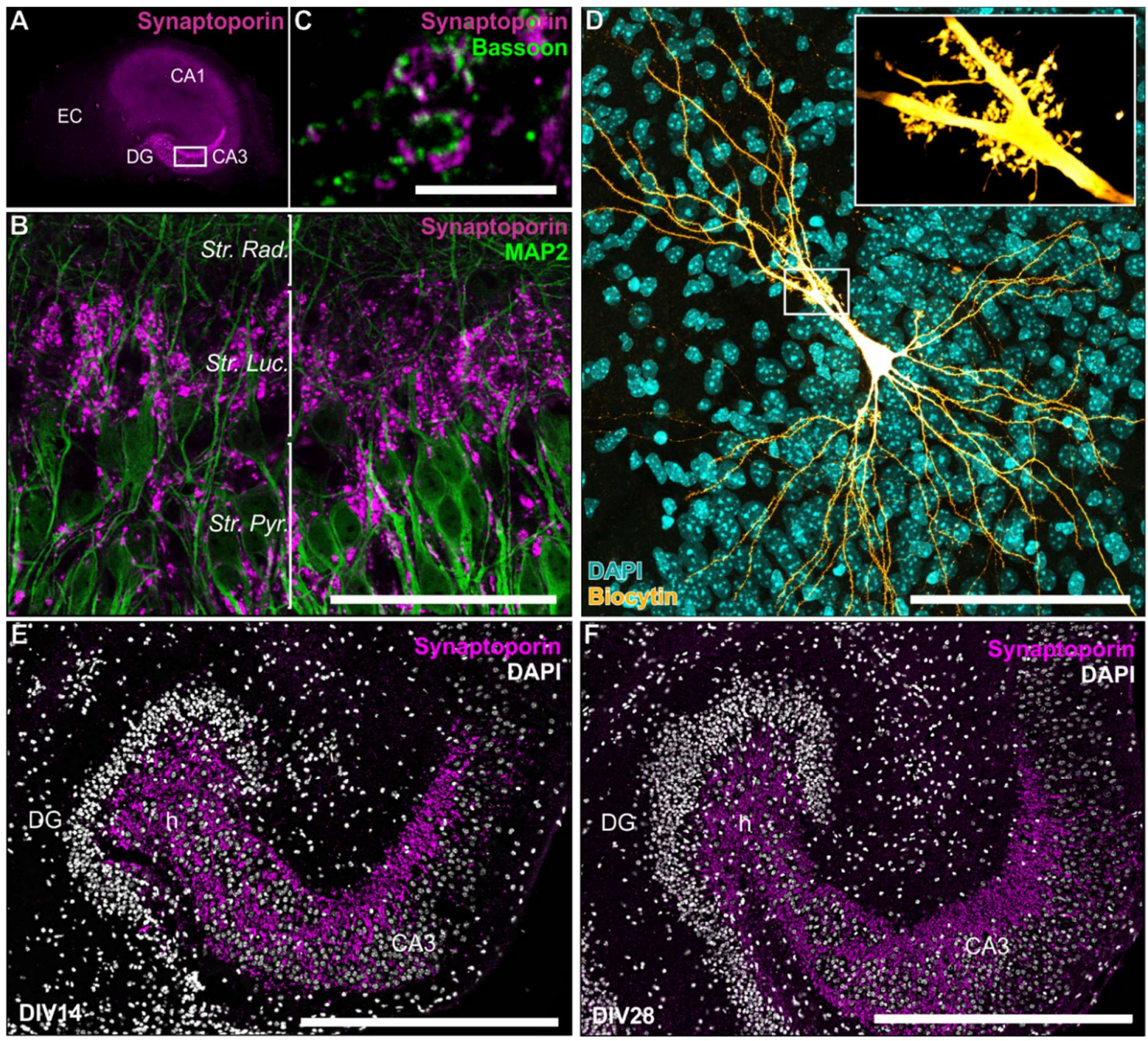

Figure 5. Light microscopic analysis of the mossy fiber-CA3 pathway in hippocampal organotypic slice cultures at DIV14 and 28.

(A) Widefield light microscopic overview of a wild-type hippocampal slice culture labelled against synaptoporin, a synaptic vesicle channel protein enriched in mossy fiber synapses. (B) Maximum projection of a confocal z-stack in which synaptoporin-labeled mossy fiber boutons (magenta) are seen to cluster around MAP-2 positive proximal dendrites (green) of pyramidal cells in CA3 stratum lucidum. (C) Single-plane confocal micrograph illustrating the high density of bassoon-labelled active zones (green) within large synaptoporin-positive mossy fiber boutons (magenta). (D) Maximum projection of a confocal z-stack through a CA3 pyramidal neuron filled with biocytin and visualized with streptavidin-conjugated Alexa 555 ("orange hot" LUT). Proximal dendrites are decorated with complex, multi-compartmental spines (thorny excrescences; insert) which are postsynaptic to mossy fiber-CA3 inputs. (E, F) Maximum projections of confocal z-stacks through organotypic slice cultures at DIV14 (E) and DIV28 (F). Synaptoporin (magenta) and DAPI (white) labelling is highly comparable at both developmental time-points, indicating that the expected target specificity of the mossy fiber-CA3 pathway remains intact throughout the analyzed culture period. No evidence of aberrant mossy fiber sprouting was observed. Abbreviations: EC, entorhinal cortex; DG, dentate gyrus; CA3, cornu ammonis area 3; CA1, cornu ammonis area 1; Str., stratum; Rad., radiatus; Luc., lucidum; Pyr., pyramidale; h, hilus; DIV, days in vitro. Scale bars: $5 \mu \mathrm{m}$, C; $100 \mu \mathrm{m}, \mathbf{B}$ and D; $500 \mu \mathrm{m}, \mathbf{E}$ and F.

contacts with hilar mossy cells, and to the stratum lucidum of the CA3, where mossy fiber boutons establish synaptic contacts with pyramidal cells. Within the CA3 stratum lucidum, 
large synaptoporin-positive puncta were observed to be clustered apposed to the primary dendrites of MAP-2-positive CA3 pyramidal neurons (Figure 5 B). To observe the distribution of active zones within mossy fiber terminals at a higher resolution, I performed a confocal light microscopic analysis of thin cryosections from cultured slices co-labelled with antibodies detecting synaptoporin and the active zone protein bassoon. Bassoon-immunoreactive puncta clustered at a high density within synaptoporin-positive mossy fiber boutons in the stratum lucidum (Figure $5 \mathrm{C}$ ). This finding indicates that mossy fiber boutons in slice cultures contain multiple active zones, which is a characteristic feature of this synapse type (Amaral and Dent, 1981; Chicurel and Harris, 1992; Rollenhagen et al., 2007). To test whether the postsynaptic organization of the mossy fiber-CA3 pyramidal cell connection is preserved in hippocampal slice cultures, biocytin-filled CA3 pyramidal neurons generated during patchclamp recordings (performed by Dr. Bekir Altas) were post-labeled using streptavidinconjugated Alexa-555. Consistent with descriptions of CA3 pyramidal neurons in vivo (Amaral et al., 2007; Chicurel and Harris, 1992; Frotscher et al., 2014; Gonzales et al., 2001), confocal light microscopic analysis revealed the presence of complex, multi-compartmental dendritic spines ("thorny excrescences") engulfing the primary dendrites of CA3 pyramidal neurons in cultured slices (Figure 5 D).

The pattern of synaptoporin immunoreactivity was highly comparable in thin cryosections from hippocampal slice cultures at DIV14 (Figure 5 E) and DIV28 (Figure 5 F), indicating that the anatomical organization of mossy fiber projection remains intact at both developmental time-points used for subsequent ultrastructural analyses. Synaptoporin-positive puncta were restricted to the hilus and stratum lucidum and no evidence of aberrant mossy fiber sprouting (Coltman et al., 1995) into the dentate granule cell layer was observed.

On the ultrastructural level (Figure 6), transmission electron micrographs of organotypic hippocampal slices exhibited an excellent preservation of synaptic ultrastructure as assessed by published criteria (Möbius et al., 2010). Consistent with previous reports in transcardially perfused rat brains (Chicurel and Harris, 1992; Rollenhagen et al., 2007), large mossy fiber boutons in the stratum lucidum formed three different types of contacts with postsynaptic CA3 pyramidal neurons, namely axo-spinous synaptic contacts onto thorny excrescences (Figure 6 B), axo-dendritic synaptic contacts onto the shaft of primary dendrites (Figure $6 \mathrm{C}$ ), 

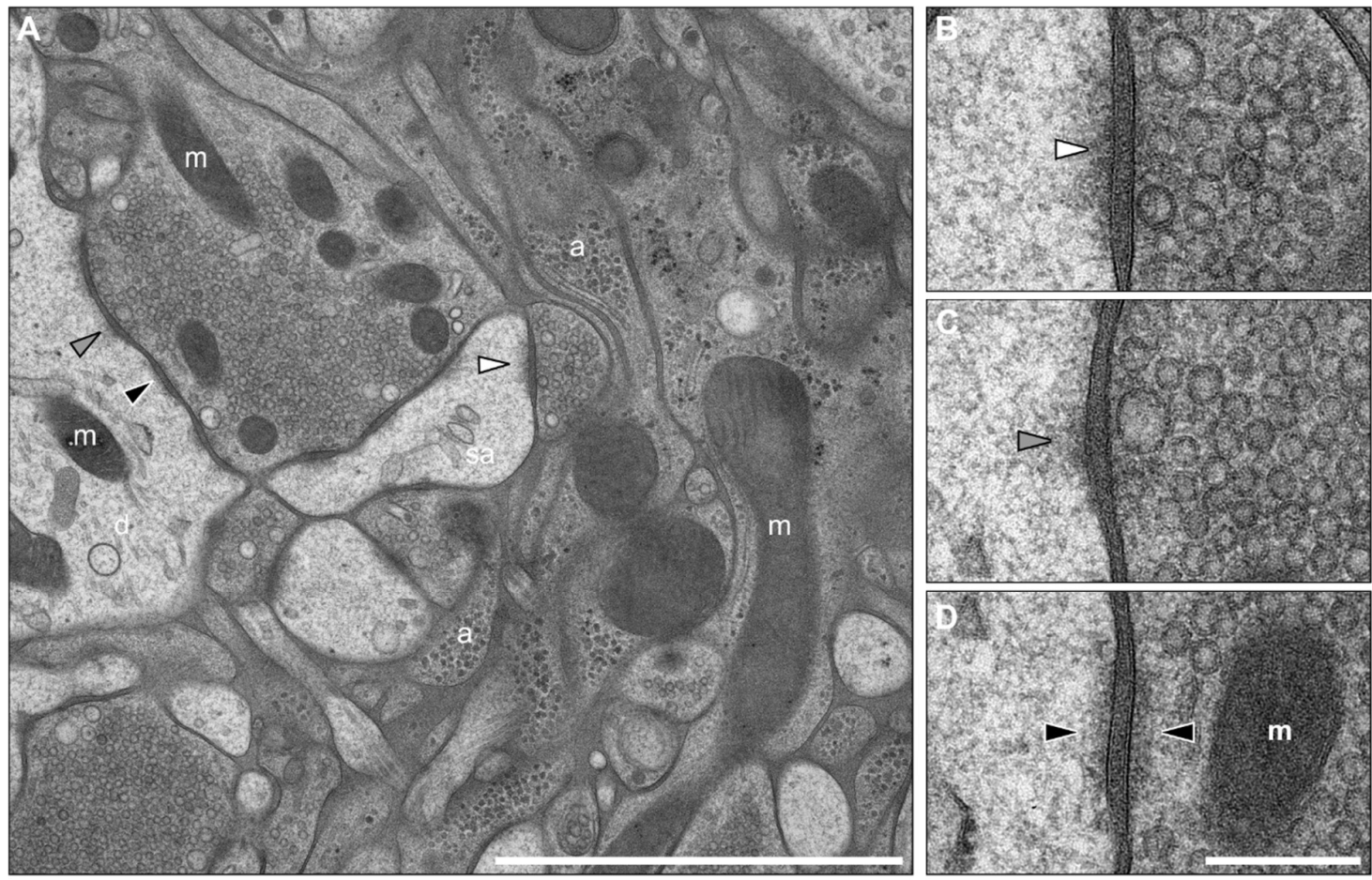

Figure 6. Ultrastructural organization of mossy fiber-CA3 synapses in organotypic hippocampal slices prepared by high-pressure freezing and freeze substitution.

(A-D) Electron micrograph of a mossy fiber bouton and CA3 pyramidal neuron from a wild-type hippocampal slice culture at DIV14. Arrowheads highlight the three contact types mossy fiber boutons make with CA3 pyramidal neurons. White arrowheads indicate synaptic contact with spines, higher magnification in (B). Dark gray arrowheads mark the synaptic contacts onto the dendritic shaft of the CA3 pyramidal neuron, higher magnification in (C). Black arrowheads indicate a puncta adherens formed between a mossy fiber bouton and the dendritic shaft of a CA3 pyramidal neuron (D). Abbreviations: $m$, mitochondria; a, astrocytic process. Scale bars: $1 \mu \mathrm{m}, \mathbf{A} ; 200 \mu \mathrm{m}$, B-D.

and puncta adherens onto the dendritic shaft (Figure 6 D). Synaptic contacts were characterized by the presence of a postsynaptic density, the widening of the synaptic cleft, and an accumulation of presynaptic vesicles in direct apposition to the postsynaptic density (Gray, 1959; Palay, 1956; Studer et al., 2014). Puncta adherentia contacts were distinguished by the presence of pre- and postsynaptic membrane specializations and a distinct absence of membrane-proximal presynaptic vesicles (Chicurel and Harris, 1992).

The ultrastructural morphology of large mossy fiber boutons in the stratum lucidum of cryofixed hippocampal slice cultures at DIV14 and mice transcardially perfused at P28 were found to be highly comparable (Figure A, B). The mossy fiber boutons in slices at DIV28 were also 

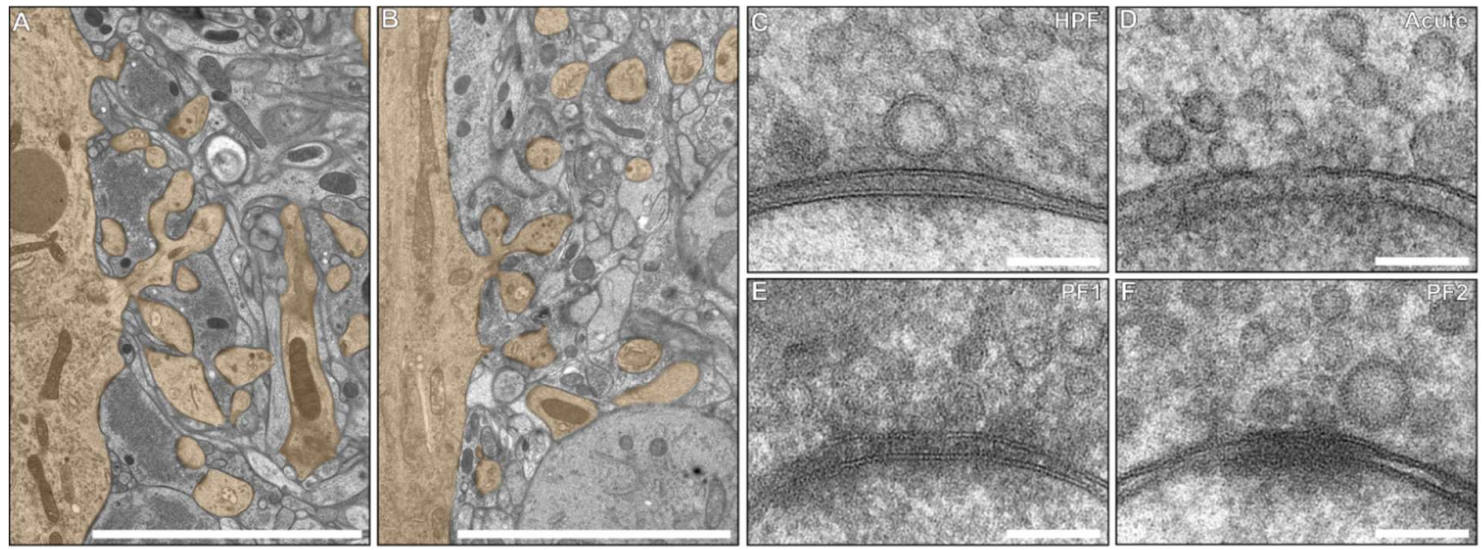

Figure 7. Comparative ultrastructural analysis of mossy fiber-CA3 synapse morphology in organotypic and ex vivo hippocampal preparations.

\begin{abstract}
(A, B) Electron micrographs in the CA3 stratum lucidum from a hippocampal slice culture (A) and perfusionfixed wild-type mouse hippocampus (B) with mossy fiber boutons engulfing complex spines of CA3 pyramidal neurons (false colored in yellow). (C-F) High magnification electron micrographs of mossy fiber-CA3 pyramidal neuron spine synapses from organotypic slice culture (C), acute slice preparation (D), and perfusion fixation (E) from Perfusion Fixative 1 (PF1; ice-cold 4\% PFA, 2.5\% glutaraldehyde in $0.1 \mathrm{M} \mathrm{PB}$; and (F) from Perfusion Fixative 2 (PF2; $37^{\circ} \mathrm{C}, 2 \%$ PFA, $2.5 \%$ glutaraldehyde, $2 \mathrm{mM} \mathrm{CaCl} 2$ in $0.1 \mathrm{M}$ cacodylate buffer). Scale bars: $5 \mu \mathrm{m}, \mathbf{A}$ and B; $100 \mathrm{~nm}, \mathbf{C}-\mathbf{F}$.
\end{abstract}

comparable to the perfused mossy fiber boutons at P28 (data not shown). A closer examination of individual mossy fiber active zone release sites in high-magnification electron micrographs revealed general structural similarities in high-pressure frozen and freezesubstituted slice cultures (Figure $7 \mathrm{C}$ ), acute slice preparations (Figure $7 \mathrm{D}$ ), and perfusionfixed hippocampi based on two seminal ultrastructural studies; Perfusion Fixative 1 (PF1; Figure $7 \mathrm{E}$; ice cold 4\% PFA, 2.5\% GA in $0.1 \mathrm{M} \mathrm{PB}$; Rollenhagen et al., 2007) and Perfusion Fixative 2 (PF2; Figure $7 \mathrm{~F} ; 37^{\circ} \mathrm{C}, 2 \% \mathrm{PFA}, 2.5 \% \mathrm{GA}, 2 \mathrm{mM} \mathrm{CaCl} 2$ in $0.1 \mathrm{M}$ cacodylate buffer; Chicurel and Harris, 1992).

In conclusion, my data demonstrate that an in vivo-like anatomical organization of the hippocampal mossy fiber projection is retained in organotypic slice cultures and that the structural characteristics of the pre- and postsynaptic compartments of the mossy fiber-CA3 synapse are exquisitely preserved for ultrastructural analysis using a combination of HPF and freeze substitution. 


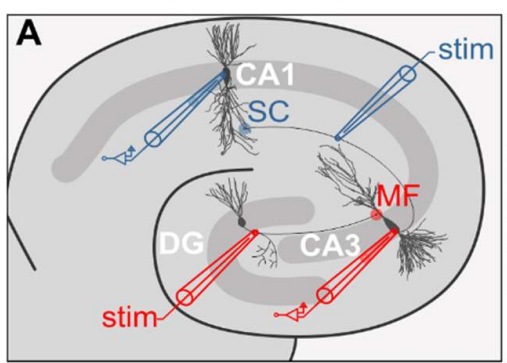

B C

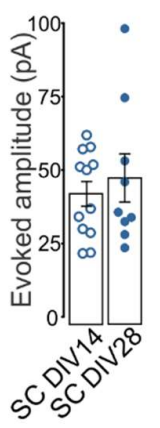

D

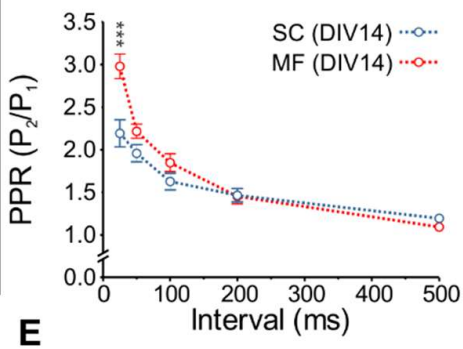

$\mathbf{F}$

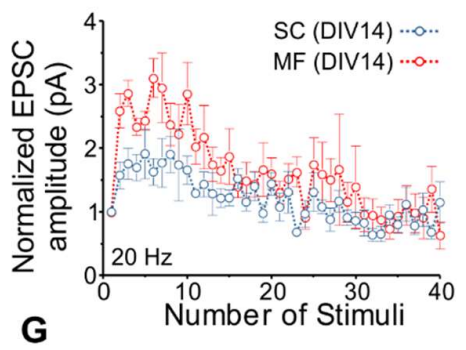

G

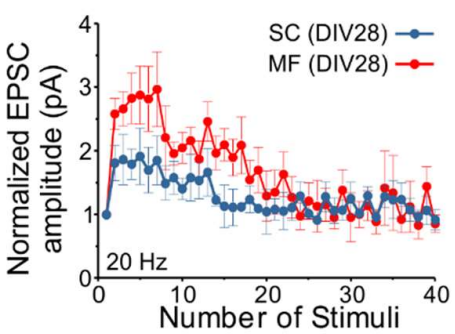

Figure 8. Comparison of Schaffer collateral and mossy fiber release probability and shortterm plasticity characteristics from hippocampal slice cultures at DIV14 and 28.

(A) Schematic of electrophysiological experiments performed on hippocampal slice cultures, in red the placement of the stimulation electrode (stim) and the patch-clamped postsynaptic cell for mossy fiber recordings, and in blue the placement of the stimulation electrode (stim) and the patch-clamped postsynaptic cell for Schaffer collateral recordings. (B) Scatter plot of the mean evoked amplitude in CA1 pyramidal neurons from Schaffer collateral synapses slice cultures at DIV14 (open circles) and DIV28 (solid circles). (C) Scatter plot of the mean evoked amplitude in CA3 pyramidal neurons from mossy fiber synapses from slice cultures at DIV14 (open circles) and DIV28 (closed circles). (D-E) Paired-pulse ratios of Schaffer collateral (blue) and mossy fiber (red) synaptic responses from slice cultures at DIV14 (D) and DIV28 (E). (FG) Normalized EPSC amplitude recorded in CA1 (blue) and CA3 (red) pyramidal neurons during a $20 \mathrm{~Hz}$ train of stimulation of Schaffer collateral and mossy fiber synapses, respectively, from slice cultures at DIV14 (F) and DIV28 (G). Abbreviations: DG, dentate gyrus; MF, mossy fiber; SC, Schaffer collateral; PPR, paired-pulse ratio. Statistical significance is represented as *, $\mathrm{p}<0.05 ; * *, p<0.005 ;{ }^{* *}, \mathrm{p}<0.001$. $\mathrm{N}=3$ cultures; $\mathrm{n}=12$ cells SC at DIV14; 9 cells SC at DIV28; 11 cells MF DIV14; 9 cells MF DIV28. See Table 15 for all statistical analyses.

\subsubsection{Comparative functional analysis of Schaffer collateral and mossy fiber synapses in hippocampal slice cultures}

To assess whether the functionally well-characterized neurotransmitter release properties of Schaffer collateral and mossy fiber-CA3 synapses described in acute hippocampal slice preparations are also preserved in cultured organotypic slices, electrophysiological recordings of postsynaptic responses were made from CA1 and CA3 pyramidal neurons during fiber stimulation of the Schaffer collateral and mossy fiber axon projections, respectively (Figure 8) performed by Chungku Lee). Whole-cell voltage-clamp recordings from CA1 pyramidal neurons were performed during fiber stimulation of Schaffer collateral axons (Figure $8 \mathrm{~A}$, 
blue). No difference in the evoked EPSC amplitude was found between DIV14 and DIV28 (Figure 8 B; $42.23 \pm 4.252$ pA, SC DIV14; $47.64 \pm 8.251$ pA, SC DIV28; p=0.54). Similarly, measurements from CA3 pyramidal neurons in response to stimulation of the mossy fiber axonal pathway in the hilus (Figure $8 \mathrm{~A}$, red) showed no significant difference in the evoked EPSC amplitude between the two developmental time-points (Figure 8 C; $93.96 \pm 9.533$ pA, MF DIV14; $101.3 \pm 19.12$ pA, MF DIV28; $p=0.71$ ). The paired-pulse ratio for mossy fiber synapses was significantly higher in response to closely spaced stimuli $(25 \mathrm{~ms}$ interstimulus interval) compared to Schaffer collateral synapses at both DIV14 (Figure 8 D; $2.203 \pm 0.116$, SC DIV14; $2.981 \pm 0.145$, MF DIV14; $p<0.001$ ) and DIV28 (Figure 8 E; $2.195 \pm 0.160$, SC DIV28; $2.712 \pm 0.169$, MF DIV28; $p=0.04$ ). Correspondingly, mossy fiber synapses demonstrated a considerably higher degree of facilitation than Schaffer collaterals in response to highfrequency fiber stimulation trains (Figure $8 \mathrm{~F}$ and G) (40 stimuli delivered at $20 \mathrm{~Hz}$ ). These synapse-specific differences in short-term plasticity were similarly observed at both DIV14 (Figure $8 \mathrm{~F}$ ) and DIV28 (Figure $8 \mathrm{G}$ ) developmental time-points. Taken together, these data indicate that fundamental and presynaptically expressed differences in release probability and short-term plasticity also exist between Schaffer collateral and mossy fiber-CA3 synapses in a cultured organotypic slice context.

\subsubsection{Comparative ultrastructural analysis of Schaffer collateral and mossy fiber synapses in hippocampal slice cultures}

To investigate whether the differences in functional release properties of Schaffer collateral and mossy fiber-CA3 synapses correlate with distinct ultrastructural profiles of synaptic vesicle organization at individual active zone release sites, I performed a comparative electron tomographic analysis of the respective synapse types. I compared Schaffer collateral and mossy fiber synapses from within the same organotypic slice to minimize experimental variability. Tomographically reconstructed active zone subvolumes from mossy fiber-CA3 (Figure $9 \mathrm{~A}$ ) and Schaffer collateral (Figure 9 D) synapses enabled accurate resolution and quantification of docked synaptic vesicles (green arrowheads) in direct contact with the presynaptic membrane. Segmented 3D models generated from the tomographic subvolumes (Figure $9 \mathrm{~B}, \mathrm{C}, \mathrm{E}, \mathrm{F}$ ) allowed for the precise extraction of the spatial coordinates of synaptic vesicles with respect to the relative position and surface area of reconstructed active zones. This permitted numbers of docked synaptic vesicles to be normalized to reconstructed active 
zone areas (Figure $9 \mathrm{C}, \mathrm{F}$ ), thereby controlling for variations in section thickness or the size of reconstructed active zones.

I first analyzed the spatial distribution of vesicles with respect to the active zone membrane. In both mossy fiber-CA3 and Schaffer collateral synapses, the majority of all vesicles within $100 \mathrm{~nm}$ of the active zone were positioned within $0-2 \mathrm{~nm}$ of the plasma membrane and therefore "docked" at the active zone (Figure $9 \mathrm{G}$ ). However, the number of docked vesicles normalized to the active zone area was significantly lower in mossy fiber synapses compared to Schaffer collateral synapses (Figure $9 \mathrm{H}$; 0-2 nm: $1.015 \pm 0.112$, SC; $0.576 \pm 0.576$; MF; $p=0.003)$. This synapse-specific difference in the abundance of membrane-proximal vesicles was also observed for vesicles within 0-5 nm of the active zone membrane (Figure 9 ।; 0-5 nm: $1.149 \pm 0.127, \mathrm{SC} ; 0.687 \pm 0.095, \mathrm{MF} ; \mathrm{p}=0.006)$. Interestingly, mossy fiber-CA3 synapses exhibited a second membrane-proximal pool of vesicles within $5-20 \mathrm{~nm}$ of the active zone membrane that was absent in Schaffer collateral synapses (Figure 9 I; 5-10 nm: 0.239 \pm 0.054 , SC; $0.471 \pm 0.076, M F ; p=0.005)$. The average active zone size sampled in reconstructed synaptic subvolumes of Schaffer collateral synapses was significantly smaller than in mossy fiber synapses (Figure 9J; $3.495 \pm 0.185, \mathrm{SC} ; 4.470 \pm 0.2600 .01 \mu \mathrm{m}^{2}, \mathrm{MF} ; \mathrm{p}=0.003$ ). A lower proportion of vesicles within $40 \mathrm{~nm}$ of the active zone was morphologically docked in mossy fiber compared to Schaffer collateral synapses (Figure 9 K; $0.471 \pm 0.040$, SC; $0.297 \pm 0.038$, MF; $p=0.002$ ). Despite such differences in their relative distribution, the number of vesicles within $40 \mathrm{~nm}$ of the active zone (Figure $9 \mathrm{~L} ; 2.137 \pm 0.137$, SC; $2.072 \pm 0.141$, MF; $\mathrm{p}=0.74$ ), and within $100 \mathrm{~nm}$ of the active zone were highly comparable between mossy fiber and Schaffer collateral synapses at DIV14 (Figure 9 M; $6.534 \pm 0.276$, SC; $6.356 \pm 0.415$, MF; $p=0.71$ ).

I similarly analyzed the distribution of vesicles at active zones from Schaffer collateral and mossy fiber synapses at DIV28 to investigate potential developmental changes in synaptic vesicle organization at the active zone. In comparison to Schaffer collateral and mossy fiber synapses at DIV14, both Schaffer collateral and mossy fiber synapses at DIV28 exhibited an increase in docked synaptic vesicles (Figure $9 \mathrm{~N}$ and 0 ). However, mossy fiber synapses persistently harbored a lower spatial density of docked vesicles (Figure $90 ; 1.710 \pm 0.139$, SC; $1.061 \pm 0.141, \mathrm{MF} ; \mathrm{p}<0.001$ ) and vesicles within $0-5 \mathrm{~nm}$ of the active zone (Figure $9 \mathrm{P} ; 1.721 \pm$ 0.138 , SC; $1.071 \pm 0.124$, MF; $p<0.001$ ) as well as the prominent accumulation of membraneproximal vesicles (Figure 9 P; 5-10 nm: $0.072 \pm 0.024$, SC; $0.332 \pm 0.055$, MF; p<0.001; $10-$ 
$20 \mathrm{~nm}: 0.100 \pm 0.033$, SC; $0.323 \pm 0.051$, MF; $p<0.001 ; 20-30 \mathrm{~nm}: 0.109 \pm 0.027$, SC; $0.242 \pm$ $0.047, M F ; p=0.03$ ) compared to Schaffer collateral synapses. Unlike synaptic subvolumes at DIV14, the active zone area sampled at DIV28 was similar in both Schaffer collateral and mossy fiber synapses (Figure $9 Q ; 4.558 \pm 0.279$, SC; $5.533 \pm 0.4000 .01 \mu \mathrm{m}^{2}$, MF; $p=0.08$ ). Consistent with my previous observation, Schaffer collateral synapses maintained a comparatively higher proportion of docked vesicles relative to all vesicles within $40 \mathrm{~nm}$ of the active zone (Figure $9 \mathrm{R} ; 0.724 \pm 0.032$, SC; $0.452 \pm 0.042 ; \mathrm{p}<0.001$ ). The total number of vesicles within $40 \mathrm{~nm}$ (Figure $9 \mathrm{~S} ; 2.312 \pm 0.148$, SC; $2.300 \pm 0.164$, MF; $\mathrm{p}=0.95$ ) and within $100 \mathrm{~nm}$ of the active zone was highly comparable between mossy fiber and Schaffer collateral synapses at DIV28 (Figure 9 T; $6.478 \pm 0.347$, SC; $6.193 \pm 0.470$, MF; $p=0.63$ ).

In conclusion, my data reveal that mossy fiber active zones harbor fewer docked vesicles than Schaffer collaterals at both DIV14 and DIV28. Interestingly, mossy fiber synapses are also characterized by a prominent membrane-proximal pool of vesicles located 5-20 nm from the active zone membrane that is not observed in wild-type Schaffer collateral synapses. This comparative disparity in vesicle organization is indicative of different spatial distributions rather than vesicle availability, since the total number of vesicles within $40 \mathrm{~nm}$ or $100 \mathrm{~nm}$ of the active zone are highly comparable in both Schaffer collateral and mossy fiber synapses. 

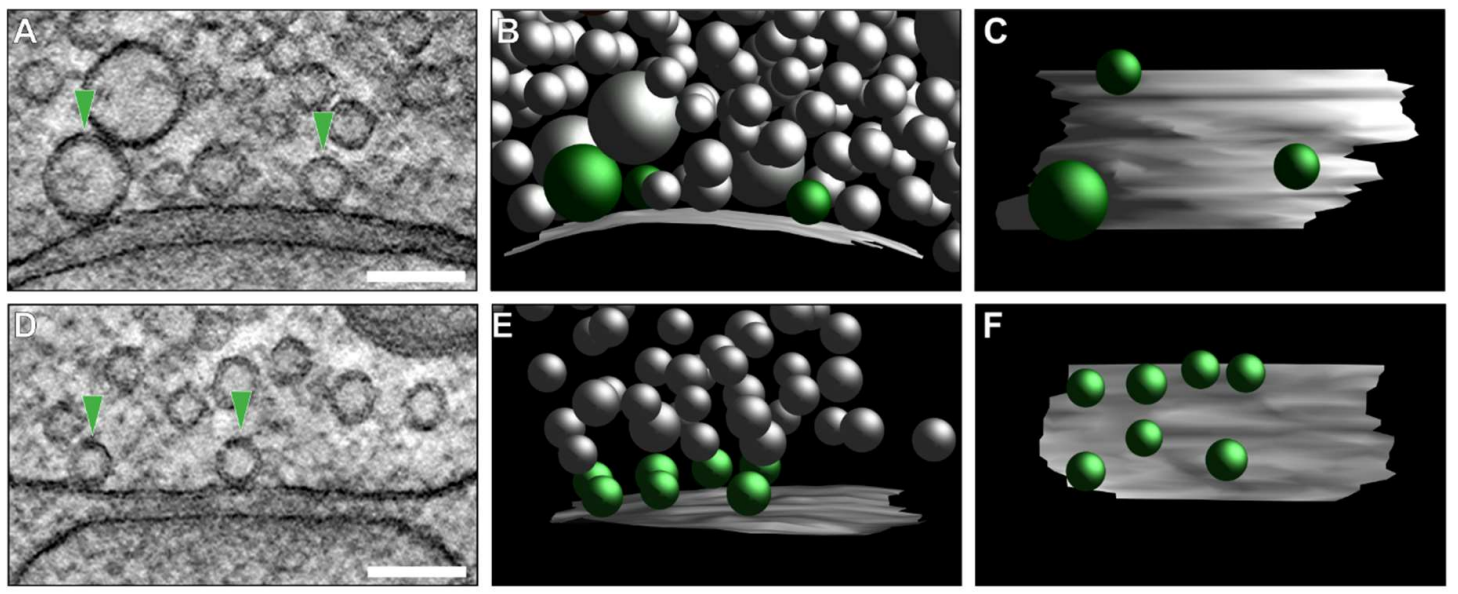

G
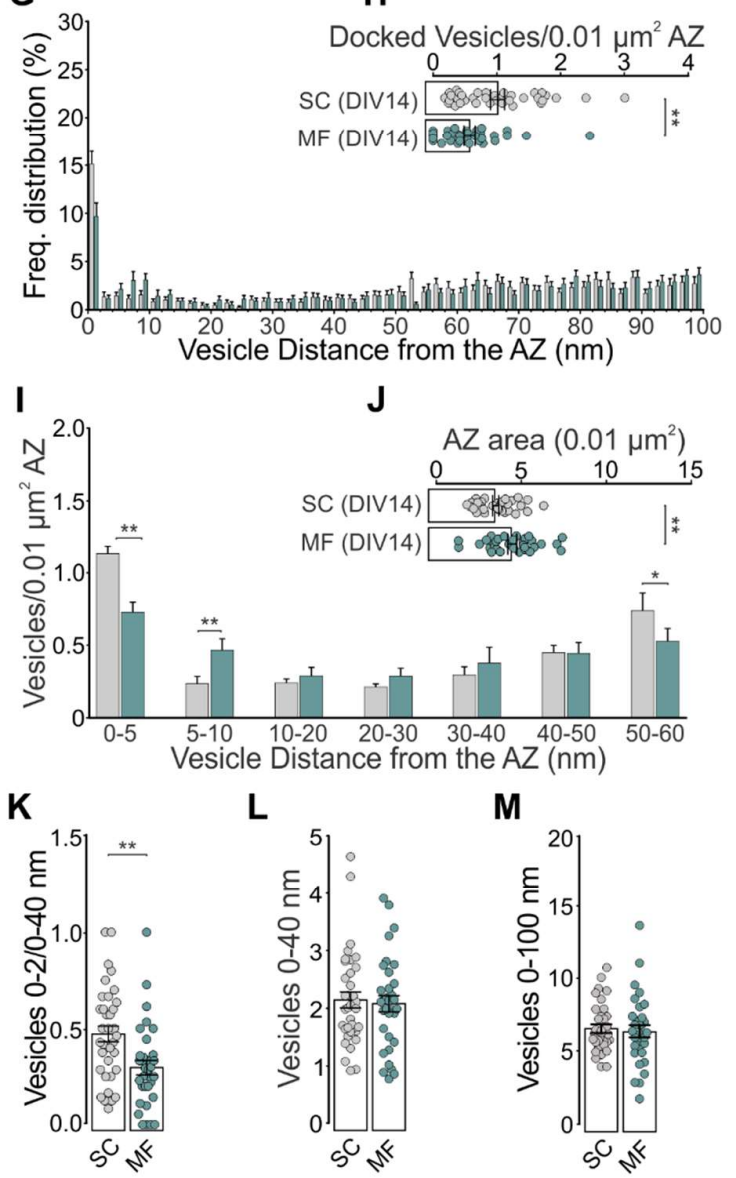

$\mathrm{L}$

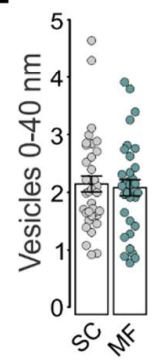

M

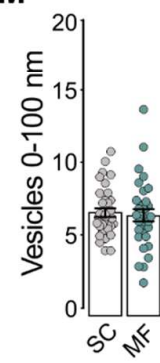

Schaffer Collateral (SC; DIV14; $N=3 ; n=36$ ) Mossy Fiber(MF; DIV14; $N=3 ; n=33$ )
$\mathbf{N}$

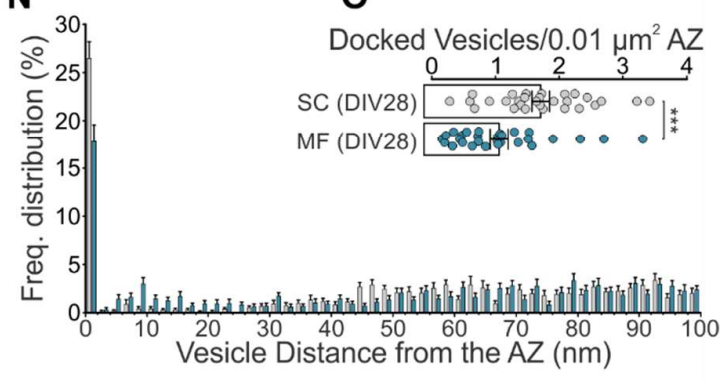

$\mathbf{P}$

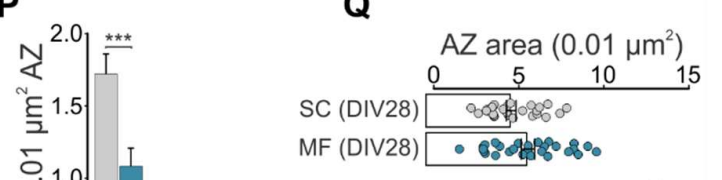

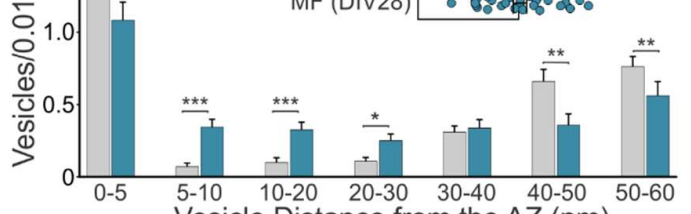

$\mathbf{R}$

$\mathbf{S}$
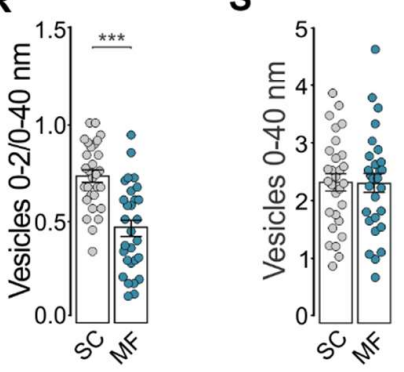

$\mathbf{T}$

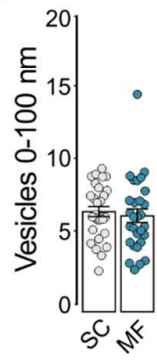

Schaffer Collateral (SC; DIV28; $N=3 ; n=29)$ Mossy Fiber (MF; DIV28; N=3; $=29$ )

Figure 9. Comparative ultrastructural analysis of Schaffer collateral and mossy fiber active zones in organotypic hippocampal slice slices at DIV14 and DIV28.

(A-M) Analysis of Schaffer collateral and mossy fiber synapses from hippocampal slice cultures at DIV14. (NT) Analysis of Schaffer collateral and mossy fiber synapses from hippocampal slice cultures at DIV28. (A, D) Tomographic subvolumes of mossy fiber (A) and Schaffer collateral (D) active zones (docked synaptic vesicles, green arrowheads). (B, E) Corresponding 3D models of mossy fiber (B) and Schaffer collateral (E) synapses (docked synaptic vesicles, green spheres; non-docked synaptic vesicles, gray spheres; active zone, 
(Figure 9. continued): active zone, gray). (C, F) Orthogonal views of the reconstructed mossy fiber (C) and Schaffer collateral (F) active zones and the spatial distribution of docked synaptic vesicles. (G, N) Frequency distribution of vesicles within $100 \mathrm{~nm}$ of the active zone membrane. $(\mathbf{H}, \mathbf{O})$ Scatter plots of docked vesicles (within 0-2 nm) at the active zone. (I, P) Spatial distribution of vesicles in 5 and $10 \mathrm{~nm}$ bins from the active zone normalized to active zone area. (J, Q) Scatter plots of active zone areas. (K, R) Scatter plots of the relative proportion of docked vesicles of all vesicles within $40 \mathrm{~nm}$ of the active zone in Schaffer collateral and mossy fiber synapses. (L, M, S, T) Scatter plots of the number of synaptic vesicles within $40 \mathrm{~nm}(\mathbf{L}, \mathbf{S})$ and $100 \mathrm{~nm}(\mathbf{M}, \mathbf{T})$ of the active zone normalized to active zone area. Statistical significance is represented as *, $\mathrm{p}<0.05 ; * *, \mathrm{p}<0.005 ; * * *, \mathrm{p}<0.001 . \mathrm{N}=$ number of cultures; $\mathrm{n}=$ number of active zones. Scale bars: $100 \mathrm{~nm}$, A-F. See Tables 15-18 for all statistical analyses for DIV14 and DIV28, respectively.

\subsubsection{Synapse-specific differences in vesicle organization characterize}

\section{Schaffer collateral and mossy fiber synapses in high-pressure frozen acute hippocampal slice preparations}

To investigate whether the difference in the density of docked vesicles at individual active zones between mossy fiber and Schaffer collateral synapses are similarly manifest in synapses which developed in vivo, I compared the ultrastructural organization of synaptic vesicle pools in Schaffer collateral and mossy fiber synapses in acute slice preparations age-matched to DIV14 slice cultures. To account for the fact that hippocampal slice cultures were prepared from mouse pups at P3-7, I prepared acute hippocampal slices from P18 wild-type mice for HPF cryofixation according to a published protocol (Korogod et al., 2015) and analyzed Schaffer collateral (Figure $10 \mathrm{~A}$ ) and mossy fiber synapses (Figure $10 \mathrm{~B}$ ) using 3D reconstructions of the synaptic subvolumes imaged with electron tomography.

As in organotypic slice cultures, the majority of all vesicles within $100 \mathrm{~nm}$ of Schaffer collateral and mossy fiber synapses were docked (within 0-2 $\mathrm{nm}$ of the active zone membrane) (Figure $10 \mathrm{C})$. Moreover, fewer docked synaptic vesicles were observed at mossy fiber synapses than at Schaffer collateral synapses when normalized to active zone area (Figure 10 D; $1.608 \pm$ 0.163 , SC; $1.128 \pm 0.095, \mathrm{MF} ; \mathrm{p}=0.009$ ), consistent with the findings in vitro (Figure $9 \mathrm{H}$ ). Although there was a trend of more vesicles within $10-20 \mathrm{~nm}$ of the active zone in mossy fiber synapses in comparison to Schaffer collateral synapses in acute preparations, this difference did not reach statistical significance (Figure $10 \mathrm{E} ; 10-20 \mathrm{~nm}$ : $0.302 \pm 0.070$, SC; $0.455 \pm 0.061$, MF; $p=0.14)$. Consistent with Schaffer collateral and mossy fiber synapses from organotypic cultures, the active zone area sampled in Schaffer collateral synapses from acute slice preparations was smaller than mossy fiber active zones from the same slice (Figure $10 \mathrm{~F} ; 3.979$ 
\pm 0.226 , SC; $5.538 \pm 0.3310 .01 \mu^{2}, M F ; p=0.001$ ). In mossy fiber synapses from acute preparations, a lower proportion of docked vesicles from all vesicles within $40 \mathrm{~nm}$ of the active zone was found in comparison to Schaffer collateral synapses (Figure $10 \mathrm{G}$; $0.617 \pm$ 0.053 , SC; $0.448 \pm 0.037, \mathrm{MF} ; \mathrm{p}=0.009$ ). Both synapse types had comparable numbers of synaptic vesicles within $40 \mathrm{~nm}$ (Figure $10 \mathrm{H} ; 2.671 \pm 0.211$, SC; $2.566 \pm 0.188$, MF; p=0.72) and $100 \mathrm{~nm}$ (Figure $10 \mathrm{l} ; 6.873 \pm 0.437, \mathrm{SC} ; 6.820 \pm 0.488, \mathrm{MF} ; \mathrm{p}=0.66$ ) of the active zone membrane.

In summary, my findings indicate that mossy fiber synapses are characterized by a comparatively lower spatial density of docked synaptic vesicles in both in vitro and in vivo preparations, thus excluding the possibility that this observation is an artifact of the slice culture system.

\subsubsection{Perfusion fixation of brain tissue causes a severe reduction in docked and membrane-proximal synaptic vesicles in mossy fiber-CA3 synapses}

Two seminal ultrastructural studies of the mossy fiber-CA3 synapse used perfusion-fixed hippocampi from rats (Chicurel and Harris, 1992; Rollenhagen et al., 2007). To test whether perfusion of chemical fixatives changed the spatial distribution of synaptic vesicles at mossy fiber synapses, I perfusion-fixed wild-type mice at P28 following the protocols from these studies (Chicurel and Harris, 1992; Rollenhagen et al., 2007). This experiment served as another ex vivo preparation to assess the abundance and spatial distribution of synaptic vesicles using electron tomography. Post-fixed brains were vibratomed and regions of the CA3 were excised with a biopsy punch before being high-pressure frozen and processed by AFS (Möbius et al., 2010). This approach was designed to focus my investigation primarily on the potential effects of aldehyde fixation on vesicle organization, rather than on shrinkage artifacts introduced by classic room-temperature dehydration steps. I found that the general morphology of mossy fiber synapses in both of the perfusion-fixed tissue to be comparable to that observed in hippocampal slice culture (Figure 7). 

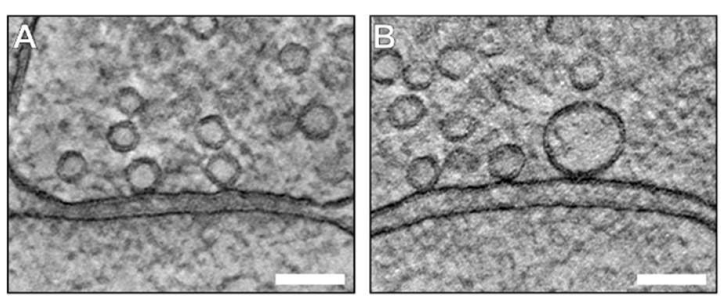

C

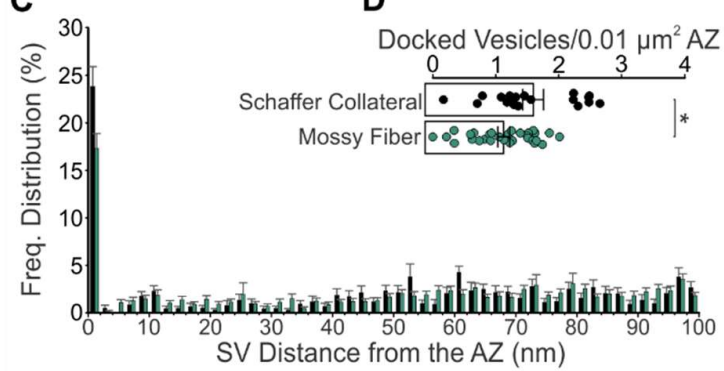

E

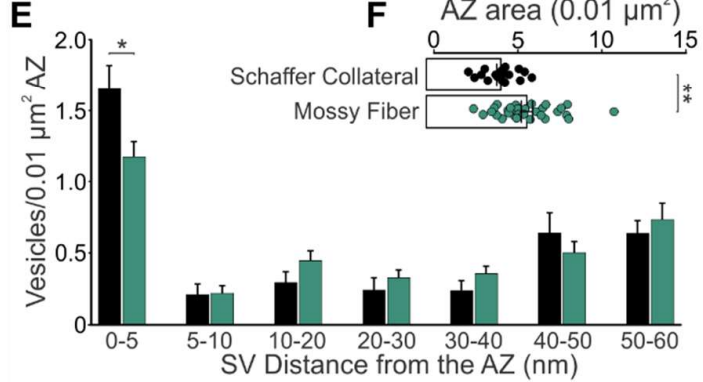

G

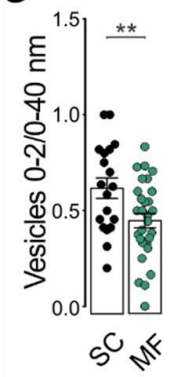

H

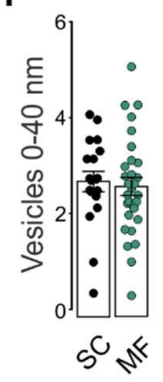

I

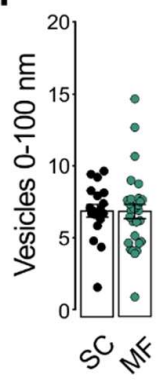

Acute P18 SC (N=3; $n=19)$ Acute P18 MF $(N=4 ; n=30)$ -
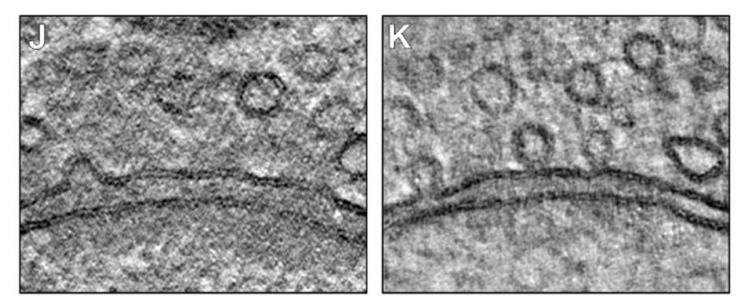

L

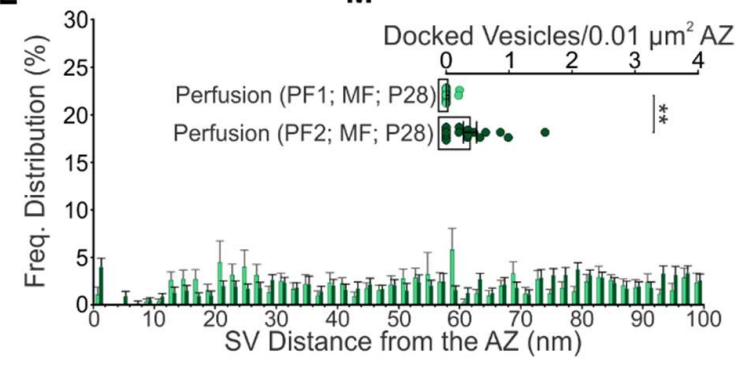

N

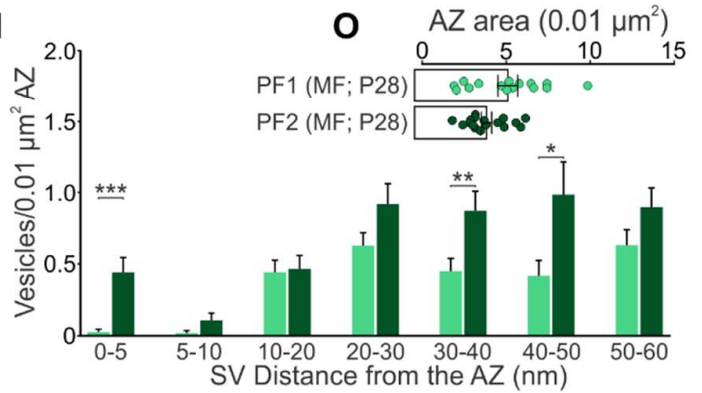

$\mathbf{P}$

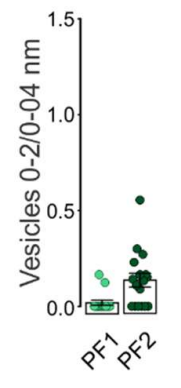

$\mathbf{R}$

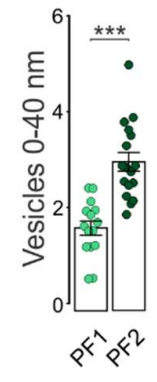

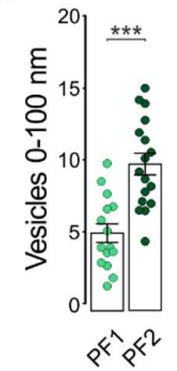

P28 (MF; $N=2 ; n=15)$ PF2 P28 (MF; N=2; n=17)

Figure 10. Ultrastructural analysis from ex vivo preparations of hippocampal tissue.

(A-I) Analysis of Schaffer collateral and mossy fiber synapses from acute hippocampal slice preparations from P18 wild-type mice. (J-R) Analysis of mossy fiber synapses from hippocampal tissue from P28 wild-type mice transcardially perfused with one of two aldehyde cocktails: PF1 (ice-cold 4\% PFA, 2.5\% GA in $0.1 \mathrm{M} \mathrm{PB}$; or PF2 ( $37^{\circ} \mathrm{C}, 2 \%$ PFA, $2.5 \% \mathrm{GA}, 2 \mathrm{mM} \mathrm{CaCl} 2$ in $0.1 \mathrm{M}$ cacodylate buffer). (A-B, J-K) Tomographic subvolumes from Schaffer collateral (A) and mossy fiber (B, J, K) synapses. (C, L) Frequency distributions of vesicles within $100 \mathrm{~nm}$ of the active zone membrane. (D, M) Scatter plots of docked vesicles $(0-2 \mathrm{~nm})$ at the active zone membrane normalized to active zone area. (E, N) Spatial distribution of vesicles in 5 and $10 \mathrm{~nm}$ bins from the active zone membrane normalized to active zone area. $(\mathbf{F}, \mathbf{O})$ Scatter plots of active zone areas. $(\mathbf{G}, \mathbf{P})$ Scatter plots of the relative proportion of docked vesicles of all vesicles within $40 \mathrm{~nm}$ of the active zone. $(\mathbf{H}$, $\mathbf{I}, \mathbf{Q}, \mathbf{R})$ Scatter plots of the number of synaptic vesicles within $40 \mathrm{~nm}(\mathbf{H}, \mathbf{Q})$ and $100 \mathrm{~nm}(\mathbf{I}, \mathbf{R})$ of the active zone normalized to active zone. Statistical significance is represented as ${ }^{*}, \mathrm{p}<0.05 ;{ }^{* *}, \mathrm{p}<0.005 ;{ }^{* * *}$, $\mathrm{p}<0.001$. $\mathrm{N}=$ number of animals; $\mathrm{n}=$ number of active zones. Scale bars: $100 \mathrm{~nm}, \mathbf{A}, \mathbf{B}, \mathbf{J}$, and $\mathbf{K}$. See Tables 1922 for full statistical analyses for acute and perfusion-fixed experiments, respectively. 
In reconstructed electron tomograms it became evident that the two fixation protocols used had stimulated vesicle fusion (Figure $10 \mathrm{~J}$ and $\mathrm{K}$ ). The two perfusion protocols were as follows: i) PF1 comprised of ice-cold 4\% PFA, 2.5\% GA dissolved in in $0.1 \mathrm{M} \mathrm{PB}$ at a pH of 7.4 (Figure $10 \mathrm{~J}$ ) (Rollenhagen et al., 2007), and ii) PF2 comprised of $2 \%$ PFA, 2.5\% GA, $2 \mathrm{mM} \mathrm{CaCl}_{2}$ dissolved in $0.1 \mathrm{M}$ cacodylate buffer at a pH of 7.4 at $37^{\circ} \mathrm{C}$ (Figure $10 \mathrm{~K}$ ) (Chicurel and Harris, 1992). The spatial distribution of synaptic vesicles was different to that observed in agematched cryo-fixed slice cultures (Figure $10 \mathrm{~L}$, perfusion fixation; Figure $9 \mathrm{~N}$, cryo-fixed wildtype slice culture). There were fewer docked synaptic vesicles in both fixation protocols compared to vesicle docking in mossy fiber synapses from acute slice preparations (Figure $10 \mathrm{M} ; 0.027 \pm 0.019, \mathrm{PF} 1 ; 0.394 \pm 0.107, \mathrm{PF} ; \mathrm{p}=0.002$; acute slice: Figure $10 \mathrm{D})$. In addition, the spatial density of synaptic vesicles in mossy fiber synapses from both perfusion fixation protocols lacked synaptic vesicles in proximity to the active zone (Figure $10 \mathrm{~N} ; 0-5 \mathrm{~nm}: 0.027$ $\pm 0.019, \mathrm{PF} 1 ; 0.486 \pm 0.103, \mathrm{PF} ; \mathrm{p}<0.001 ; 5-10 \mathrm{~nm}: 0.021 \pm 0.015, \mathrm{PF} 1 ; 0.139 \pm 0.052, \mathrm{PF} 2$; $\mathrm{p}=0.05$ ). The density of synaptic vesicles increased beyond $20 \mathrm{~nm}$ from the active zone (Figure $10 \mathrm{~N} ; 20-30$ nm: $0.629 \pm 0.090$, PF1; $0.956 \pm 0.143$, PF2; p=0.09; 30-40 nm: $0.450 \pm 0.089, P F 1 ;$ $0.871 \pm 0.137, P F 2 ; p=0.007)$. This is likely due to a smaller active zone area measured in the PF2 condition compared to PF1 and age-matched cryo-fixed slice cultures (Figure $100 ; 5.122$ $\pm 0.5920 .01 \mu \mathrm{m}^{2}$, PF1; $\left.3.854 \pm 0.3070 .01 \mu \mathrm{m}^{2}, \mathrm{PF} 2 ; \mathrm{p}=0.06\right)$. The proportion of docked vesicles from all vesicles within $40 \mathrm{~nm}$ was very low in both perfusion conditions (Figure $10 \mathrm{P}$ $0.019 \pm 0.013$, PF1; $0.137 \pm 0.036$, PF2; $p=0.003$ ), which was far below the ratio observed in mossy fiber synapses from age-match wild type slice cultures (Figure 9 R; DIV28). There were, however, more synaptic vesicles in PF2 within $40 \mathrm{~nm}$ (Figure 10 Q; $1.573 \pm 0.150$, PF1; 2.954 $\pm 0.195, \mathrm{PF} 2 ; \mathrm{p}<0.001$ ) and $100 \mathrm{~nm}$ (Figure $10 \mathrm{R} ; 4.887 \pm 0.651, \mathrm{PF} 1 ; 9.678 \pm 0.754, \mathrm{PF}$; $p<0.001$ ) than in PF1, likely as a result of the smaller active zone area samples in PF2, used for normalization.

These findings indicate that perfusion fixation of different aldehyde cocktails caused severe reductions in membrane-proximal vesicle pools in hippocampal mossy fiber synapses. The omega-shaped exo-endocytic intermediates observed in these conditions indicate that the observed depletion of membrane-attached and membrane-proximal vesicles is likely due to induced vesicle fusion. 


\subsubsection{Munc13 priming molecules are essential for vesicle docking in hippocampal mossy fiber synapses}

Previous studies have demonstrated that Munc13 priming molecules are required for morphological docking of synaptic vesicles at small, glutamatergic spine synapses in the mouse hippocampus (Imig et al., 2014; Siksou et al., 2009a). These data, together with work in invertebrate model systems (Böhme et al., 2016; Hammarlund et al., 2007; Weimer et al., 2006), indicate that this molecular requirement is evolutionarily conserved. Nevertheless, the discovery that some functionally and structurally specialized synapses, including retinal photoreceptor ribbon synapses (Cooper et al., 2012) and cochlear hair cell ribbon synapses (Vogl et al., 2015) function in the absence of Munc13-mediated priming proteins, emphasizes the importance of investigating the molecular requirements for synapse function on a subtype specific basis. Although electrophysiological studies have investigated the roles of individual Munc13 isoforms in presynaptic forms of synaptic plasticity at the mossy fiber-CA3 synapse (Breustedt et al., 2010; Yang and Calakos, 2011), the role of Munc13 priming molecules in synaptic vesicle organization at these synapses remains unclear since electron microscopic analyses have thus lacked the resolution to accurately quantify membraneattached vesicles (Zhao et al., 2012a, 2012b).

To examine the role of Munc13 proteins in synaptic vesicle docking in hippocampal mossy fiber synapses, I generated slice cultures from Munc13-1/2 DKO (Munc13-1\%, Munc13-2/-) and CTRL littermates (Munc13-1+/-, Munc13-2/-). To circumvent problems associated with the perinatal lethality of the Munc13-1 constitutive KO (Augustin et al., 1999), slice cultures were prepared from E18 mouse pups. The gross morphology of Munc13-deficient hippocampal mossy fibers appeared highly comparable to controls and gave no indication of developmental deficits, consistent with previous studies (Augustin et al., 1999; Sigler et al., 2017). In line with past studies (Imig et al., 2014; Siksou et al., 2009a), an analysis of electron tomograms from Munc13-1/2 CTRL (Figure 11 A, B, F) and DKO (Figure 11 C, D, F) mossy fiberCA3 synapses revealed a complete loss of vesicle docking as indicated by the scarcity of vesicles within both 0-2 $\mathrm{nm}$ (Figure $11 \mathrm{~F}$; Munc13s, $0.861 \pm 0.159$, CTRL; $0 \pm 0$, DKO; $p<0.001$ ) and 0-5 nm (Figure $11 \mathrm{G} ; 0-5 \mathrm{~nm}: 0.923 \pm 0.165, \mathrm{CTRL} ; 0 \pm 0$, DKO; $\mathrm{p}<0.001$ ) of the active zone 


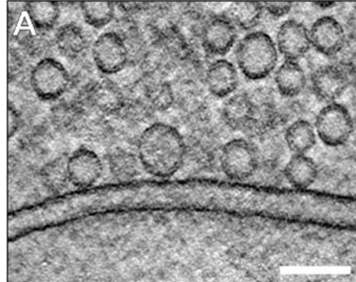

E

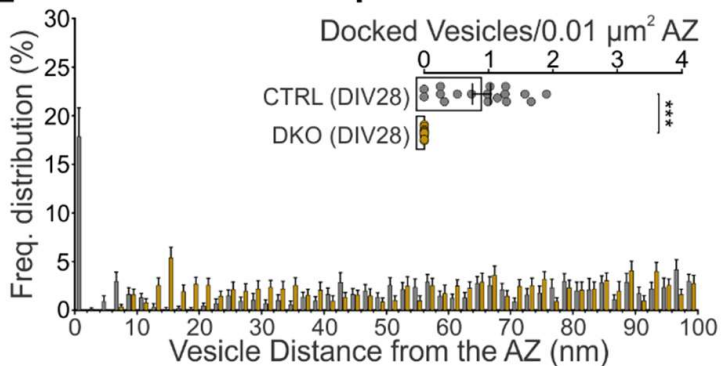

I

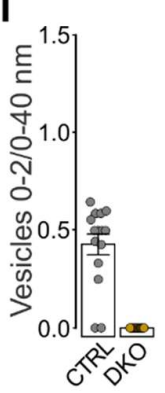

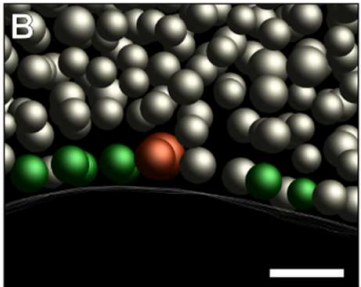

$\mathbf{F}$

J

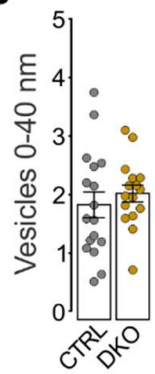

$\mathrm{K}$

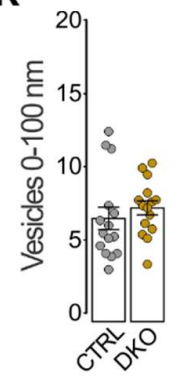

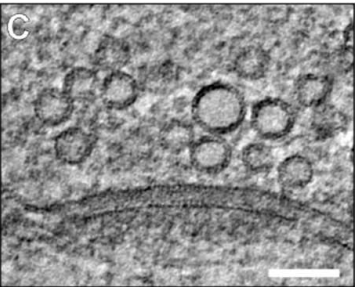

G

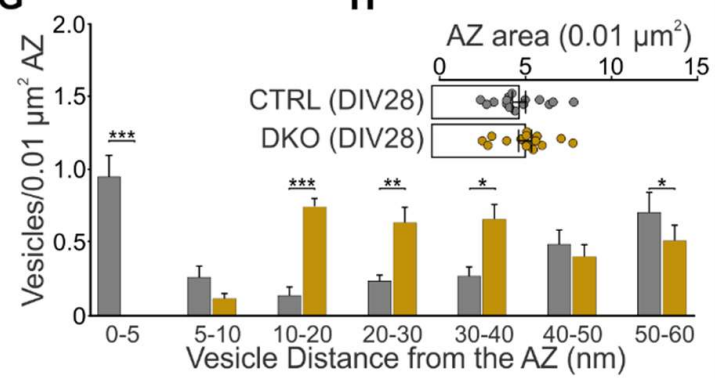

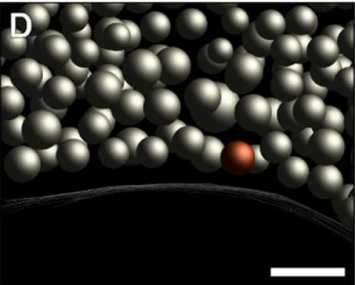

H

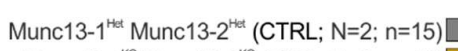
Munc13-1 ${ }^{\mathrm{KO}}$ Munc13-2 ${ }^{\mathrm{KO}}$ (DKO; N=2; n=15) $\square$

Figure 11. Ultrastructural analysis of mossy fiber active zones in Munc13-deficient and control slice cultures at DIV28.

(A, C) Tomographic subvolumes of mossy fiber synapses from Munc13-1/2 CTRL (A; Munc13-1/- Munc13$2^{+/-}$) and DKO (C; Munc13-1 ${ }^{--}$Munc13-2 ${ }^{-/}$) slice cultures. (B, D) Corresponding 3D models of CTRL (B) and DKO (D) mossy fiber synapses (docked synaptic vesicles, green; DCVs, orange; non-docked synaptic vesicles, gray; active zone, gray). (E) Frequency distribution of vesicles within $100 \mathrm{~nm}$ of the active zone. (F) Scatter plot of docked synaptic vesicles $(0-2 \mathrm{~nm})$ normalized to the active zone area. (G) Spatial distribution of vesicles in 5 and $10 \mathrm{~nm}$ bins normalized to active zone area. (H) Scatter plot of active zone areas. (I) Scatter plot of the relative proportion of docked vesicles from all vesicles within $40 \mathrm{~nm}$ of the active zone. (J, K) Scatter plots of the number of vesicles within $40 \mathrm{~nm}(\mathrm{~J})$ and $100 \mathrm{~nm}(\mathbf{K})$ of the active zone normalized to active zone area. Scale bars: $100 \mathrm{~nm}, \mathbf{A}-\mathbf{D}$. Statistical significance is represented as *, $\mathrm{p}<0.05 ;{ }^{* *}, \mathrm{p}<0.005$; $* * *, p<0.001$. $N=$ number of cultures; $n=$ number of active zones. See Table 23 and 24 for full statistical analysis.

membrane. Munc13-1/2 DKO mossy fiber synapses also exhibited a prominent accumulation of membrane-proximal vesicles between 10-40 nm from the active zone (Figure $11 \mathrm{G} ; 10-20$ $\mathrm{nm}: 0.155 \pm 0.068, \mathrm{CTRL} ; 0.762 \pm 0.059, \mathrm{DKO} ; \mathrm{p}<0.001 ; 20-30 \mathrm{~nm}: 0.224 \pm 0.048, \mathrm{CTRL} ; 0.700$ \pm 0.115 , DKO; $p=0.002 ; 30-40 \mathrm{~nm}: 0.305 \pm 0.067$, CTRL; $0.633 \pm 0.106, \mathrm{DKO} ; \mathrm{p}=0.01$ ). The active zone area sampled from CTRL and DKO synaptic subvolumes was the same (Figure $11 \mathrm{H} ; 4.615$ $\pm 0.387, \mathrm{CTRL} ; 4.976 \pm 0.3790 .01 \mu \mathrm{m}^{2}, \mathrm{MF} ; \mathrm{p}=0.51$ ). In CTRL tomograms, the density of docked vesicles (Figure 11 F, CTRL; Figure 90 , wild-type) and the proportion of docked vesicles from 
all vesicles within $40 \mathrm{~nm}$ of the active zone (Figure 11 I, CTRL, and Figure $9 \mathrm{R}$, wild-type) were highly comparable to wild-type mossy fiber synapses at DIV28. Despite severe docking deficits, vesicle recruitment within $40 \mathrm{~nm}$ (Figure $11 \mathrm{~J}$; $1.903 \pm 0.253, \mathrm{CTRL}$; $2.303 \pm 0.156$, DKO; $p=0.19$ ) and $100 \mathrm{~nm}$ (Figure $11 \mathrm{~K} ; 5.983 \pm 0.728, \mathrm{CTRL} ; 6.499 \pm 0.461$, DKO; $\mathrm{p}=0.55$ ) of the active zone was unaffected in Munc13-1/2 DKO mossy fiber synapses.

My findings indicate that Munc13s are essential for the morphological docking of vesicles at the mossy fiber synapses. Despite the severe docking deficit in Munc13-1/2 DKO mossy fiber synapses, the number of vesicles within $40 \mathrm{~nm}$ of the active zone was the same as in CTRL mossy fiber synapses. Furthermore, the density of docked vesicles in Munc13 CTRL mossy fiber synapses was the same as in wild-type mossy fiber synapses at DIV28. Despite the heterozygous genotype of Munc13-1 and -2, this was enough to achieve wild-type-like vesicle docking in mossy fiber synapses.

\subsubsection{Acute pharmacological inhibition of action potential firing does not alter docked synaptic vesicle density in mossy fiber synapses}

To address whether endogenous synaptic transmission from mossy fiber synapses influenced the spatial distribution of synaptic vesicles at the active zone, activity was blocked in wildtype hippocampal slices by an acute pharmacological treatment prior to high-pressure freeze fixation. Slices in this experiment were exposed to one of three conditions: (i) a VC, in which slices were treated with their own medium; (ii) T/N/A: a cocktail designed to block slice activity, containing TTX (T) to prevent sodium-propagated action potentials (Narahashi et al., 1964), and the postsynaptic glutamate receptor blockers NBQX (N) and D-AP5 (A) to inhibit AMPA and NMDA receptors, respectively; and (iii) T/D: a cocktail designed to selectively suppress mossy fiber synaptic transmission, comprising TTX (T) with the mGluR2 receptor agonist DCG-IV (D), which reduces synaptic transmission from mossy fiber synapses via reduction of intracellular cAMP levels (Kamiya and Ozawa, 1999; Kamiya et al., 1996). The spatial distribution of synaptic vesicles within $100 \mathrm{~nm}$ of mossy fiber active zones was unchanged after acute pharmacological silencing of network activity with both T/N/A and T/D treatment compared to VC (Figure $12 \mathrm{~A}$ ). The density of docked vesicles was comparable between VC and pharmacologically silenced mossy fiber synapses (Figure 12 B; $0.634 \pm 0.110$, VC; $0.605 \pm 0.066, T / N / A ; 0.688 \pm 0.101, T / D ; p=0.82)$. In synapses treated with T/D, vesicle numbers were increased within 0-5 nm (Figure 12 C; 0-5 nm: 0.734 \pm 0.119 , VC; $0.758 \pm 0.077$, 
$\mathrm{T} / \mathrm{N} / \mathrm{A} ; 0.946 \pm 0.118, \mathrm{~T} / \mathrm{D} ; \mathrm{p}=0.31$ ), 5-10 nm (Figure $12 \mathrm{C} ; 5-10 \mathrm{~nm}: 0.519 \pm 0.112, \mathrm{VC} ; 0.531$ $\pm 0.090, T / N / A ; 0.689 \pm 0.080, T / D ; p=0.08$ ), and 10-20 nm (Figure $12 \mathrm{C} ; 10-20 \mathrm{~nm}: 0.411 \pm$ $0.109, V C ; 0.333 \pm 0.071, T / N / A ; 0.483 \pm 0.082, T / D ; p=0.33$ ) from the active zone, however, this trend did not reach statistical significance. The active zone area measured in synaptic subvolumes from the three treatment conditions was comparable (Figure 12 D; $3.506 \pm 0.252$, VC; $3.752 \pm 0.259, T / N / A ; 3.964 \pm 0.2950 .01 \mu m^{2}, T / D ; p=0.52$ ). Despite the mild increase in membrane-proximal vesicles after acute application of T/D, the proportion of docked vesicles to all vesicles within $40 \mathrm{~nm}$ of the mossy fiber active zone was highly comparable to T/N/Atreated mossy fiber synapses and VCs (Figure $12 \mathrm{E} ; 0.265 \pm 0.051, \mathrm{VC} ; 0.261 \pm 0.030, \mathrm{~T} / \mathrm{N} / \mathrm{A}$; $0.269 \pm 0.032, T / D ; p=0.99)$. There was no change in the density of synaptic vesicles within $40 \mathrm{~nm}$ (Figure $12 \mathrm{~F} ; 2.318 \pm 0.261$, VC; $2.389 \pm 0.181, \mathrm{~T} / \mathrm{N} / \mathrm{A} ; 2.622 \pm 0.142, \mathrm{~T} / \mathrm{D} ; \mathrm{p}=0.12$ ) or $100 \mathrm{~nm}$ (Figure $12 \mathrm{G} ; 6.763 \pm 0.609, \mathrm{VC} ; 6.868 \pm 0.416, \mathrm{~T} / \mathrm{N} / \mathrm{A} ; 6.390 \pm 0.419, \mathrm{~T} / \mathrm{D} ; \mathrm{p}=0.62$ ) after acute pharmacological blockade of network activity and of mossy fiber synaptic transmission when compared to control. As expected, mossy fiber synapses exhibited a tendency towards fewer morphological fusion events after T/N/A and T/D treatments, although this reduction did not reach statistical significance with the sample size of this study (data not shown; $0.064 \pm 0.027, V C ; 0.046 \pm 0.020, T / N / A ; 0.016 \pm 0.011, T / D ; p=0.34$ ).

Taken together, these data indicate that the spatial organization of synaptic vesicles at mossy fiber active zones are rather insensitive to acute manipulations of spontaneous slice activity. The comparatively lower numbers of docked vesicles observed in mossy fiber synapses compared to Schaffer collateral synapses is therefore unlikely to result from the potential hyper-excitability of the mossy fiber pathway in slice cultures. 
A

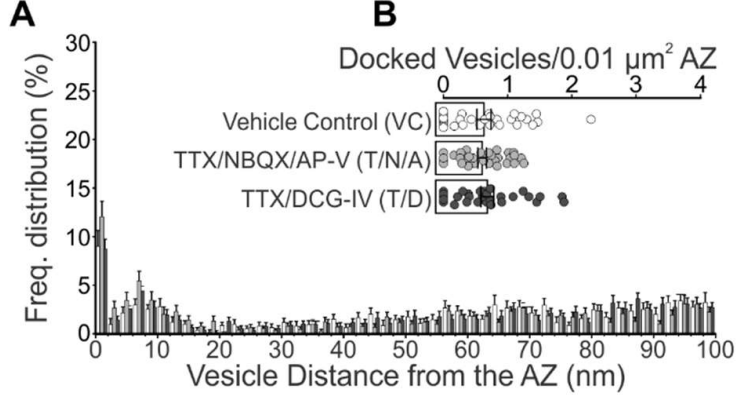

E

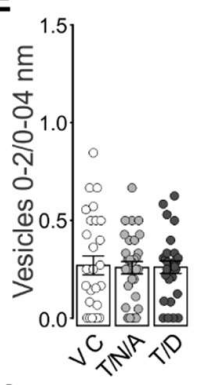

H

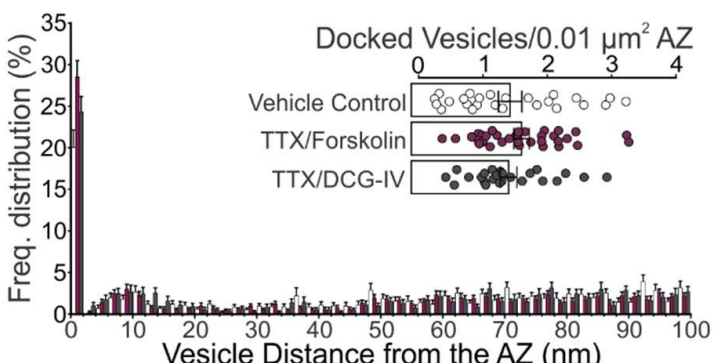

L

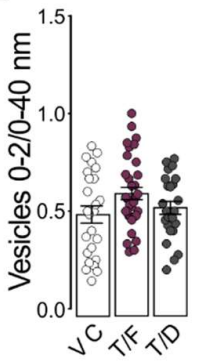

$\mathbf{F}$

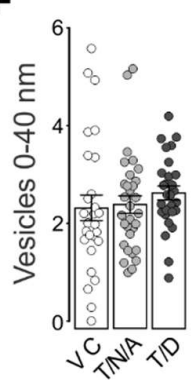

I

M

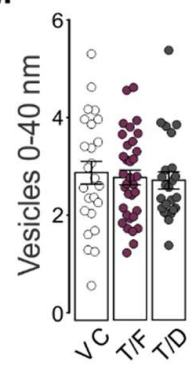

G

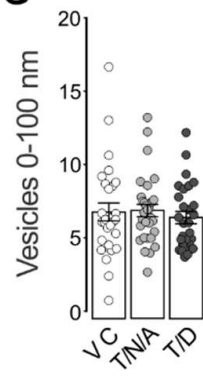

J

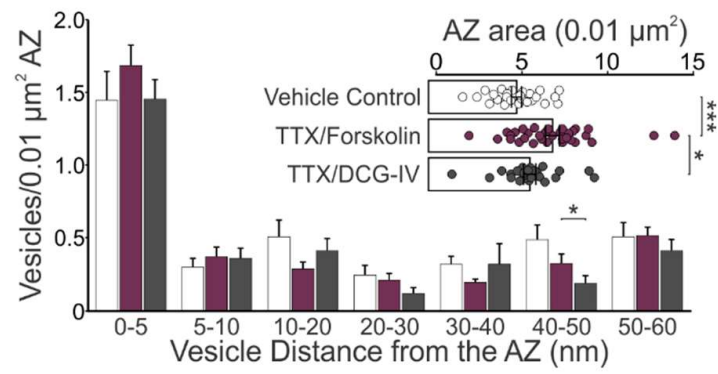

$\mathrm{N}$

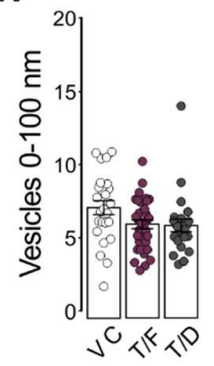

C

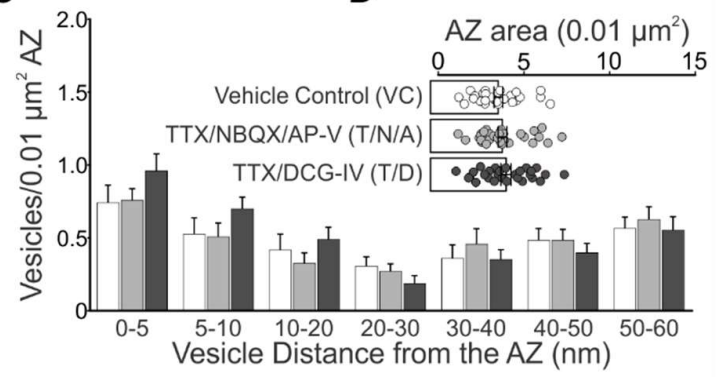

Vehicle Control (VC; $N=4 ; n=27)$ TTX/NBQX/AP-V (T/N/A; N=4; n=31) TTX/DCG-IV (T/D; $N=4 ; n=28)$

Figure 12. Ultrastructural analysis of vesicle pools after acute pharmacological manipulations of mossy fiber synapses in organotypic slice cultures at DIV14 and DIV28.

(A-G) Analysis of mossy fiber synapses after a 10 minute acute pharmacological silencing of slice cultures at DIV14 with one of three conditions: 1) T/N/A: TTX, to block sodium-propagated action potentials, supplemented with either NBQX and D-AP5 to block AMPA and NMDA receptor blockers, respectively; 2) T/D: TTX with DCG-IV, an mGluR2 receptor agonist that specifically blocks synaptic transmission in mossy fiber synapses (Kamiya and Ozawa, 1999); and 3) VC: comprised of slice culture medium. (H-N) Analysis of mossy fiber synapses from hippocampal slice cultures at DIV28 after a 15 minute treatment with either: 1) T/F: TTX, to block sodium propagated action potentials, and forskolin, an activator of AC1 which increases presynaptic CAMP concentrations (Chavez-Noriega and Stevens, 1994; Dixon and Atwood, 1989; Seamon et al., 1983) thus increasing synaptic transmission (Evans and Morgan, 2003; Seino and Shibasaki, 2005; 
(Figure 12. continued): Weisskopf et al., 1994); 2) T/D: TTX with DCG-IV, and mGluR2 receptor agonist that specifically blocks synaptic transmission in mossy fiber synapses (Kamiya and Ozawa, 1999); and 3) VC: comprised of TTX in slice culture medium. (A, H) Frequency distributions of vesicles within $100 \mathrm{~nm}$ of the active zone membrane. $(B, I)$ Scatter plots of docked vesicles $(0-2 \mathrm{~nm})$ at the active zone membrane normalized to active zone area. (C, J) Spatial distribution of vesicles in 5 and $10 \mathrm{~nm}$ bins normalized to active zone area. (D, K) Scatter plots of active zone area. (E, L) Scatter plots of the relative proportion of docked vesicles within $40 \mathrm{~nm}$ of the active zone. $(\mathbf{F}, \mathbf{G}, \mathbf{M}, \mathbf{N})$ Scatter plots of the number of synaptic vesicles within $40 \mathrm{~nm}(\mathbf{F}, \mathbf{M})$ and $100 \mathrm{~nm}$ $(\mathbf{G}, \mathbf{N})$ of the active zone normalized to active zone area. Statistical significance is represented as $*, p<0.05 ; * *$, $\mathrm{p}<0.005 ; * * *, p<0.001$. $\mathrm{N}=$ number of cultures; $\mathrm{n}=$ number of active zones. See Tables $25-26$ and $27-28$ for full statistical analyses of acutely silenced and acute pharmacological manipulation of cAMP experiments, respectively.

\subsubsection{Pharmacological manipulation of presynaptic cAMP only minimally impacts synaptic vesicle organization in mossy fiber synapses}

To investigate whether induced changes in presynaptic cAMP concentrations are reflected by corresponding changes in synaptic vesicle organization at mossy fiber active zones, I exploited the well-studied presynaptic cAMP-dependence of mossy fiber synaptic release probability (Evans and Morgan, 2003; Seino and Shibasaki, 2005; Weisskopf et al., 1994). I treated wildtype slice cultures at DIV28 with drug cocktails to either increase or decrease presynaptic cAMP concentrations. Three conditions were analyzed in this experiment: 1) T/F: TTX, to block sodium propagated action potentials, and forskolin, an activator of AC1 which increases presynaptic CAMP concentrations (Chavez-Noriega and Stevens, 1994; Dixon and Atwood, 1989; Seamon et al., 1983), thus increasing synaptic transmission (Evans and Morgan, 2003; Seino and Shibasaki, 2005; Weisskopf et al., 1994); 2) T/D: TTX with DCG-IV, an mGluR2 receptor agonist that specifically blocks synaptic transmission in mossy fiber synapses (Kamiya and Ozawa, 1999); and 3) VC: comprised of TTX in slice culture medium. Both forskolin and DCG-IV were effective in modulating spontaneous transmitter release in mossy fiber synapses in a 15-minute time frame, which was validated via electrophysiology (recordings by Bekir Altas from DIV14 slice cultures; data not shown). Slice cultures were then fixed and processed accordingly: high-pressure freeze-fixation, AFS, plastic embedding, and 3D electron tomography. The spatial distribution of synaptic vesicles within $100 \mathrm{~nm}$ of the active zone was comparable between the three pharmacological treatments (Figure $12 \mathrm{H}$ ). After increasing presynaptic mossy fiber cAMP levels with forskolin, there was a mild increase in docked synaptic vesicle density, although this tendency did not reach statistical significance (Figure $12 \mathrm{I} ; 1.422 \pm 0.183$, VC; $1.601 \pm 0.118$, T/F; $1.399 \pm 0.129, \mathrm{~T} / \mathrm{D} ; \mathrm{p}=0.40$ ). Similarly, forskolin-treated mossy fiber synapses exhibited a mild increase in synaptic vesicles within 0- 
$5 \mathrm{~nm}$ of the active zone, normalized to the active zone area, however this tendency also did not reach statistical significance (Figure $12 \mathrm{~J} ; 0-5 \mathrm{~nm}: 1.456 \pm 0.191, \mathrm{VC} ; 1.692 \pm 0.135, \mathrm{~T} / \mathrm{F}$; $1.463 \pm 0.127, T / F ; p=0.43)$. The second pool of membrane-proximal synaptic vesicles was unaffected by manipulation of presynaptic CAMP (Figure $12 \mathrm{H}$ and J). There was an increase in the proportion of docked synaptic vesicles from all vesicles within $40 \mathrm{~nm}$ of the active zone after increasing presynaptic CAMP levels with forskolin that was reminiscent to the proportion observed at untreated Schaffer collateral synapses at DIV28 (Figure 12 L, $0.482 \pm 0.044$, VC; $0.589 \pm 0.031, T / F ; 0.517 \pm 0.033, T / D ;$ Figure 9 R, untreated DIV28 Schaffer collateral). The number of synaptic vesicles within $40 \mathrm{~nm}$ (Figure $12 \mathrm{M} ; 2.871 \pm 0.233$, VC; $2.775 \pm 0.151$, T/F; $2.713 \pm 0.178, T / D ; p=0.85$ ) and $100 \mathrm{~nm}$ of the active zone membrane (Figure $12 \mathrm{~N} ; 7.047 \pm$ $0.473, V C ; 5.912 \pm 0.299, T / F ; 5.829 \pm 0.438, T / D ; p=0.03$ ) was unchanged after manipulation of presynaptic CAMP in mossy fiber synapses.

These findings indicate that acute pharmacological manipulation of presynaptic cAMP concentrations in mossy fiber synapses does not have a major impact on synaptic vesicle organization at the active zone. Rather, my data indicate that forskolin-induced enhancement of synaptic transmission acts via molecular mechanisms operating downstream of synaptic vesicle docking and priming. 


\subsection{Morphological heterogeneity of the docked vesicle pool in mossy fiber synapses}
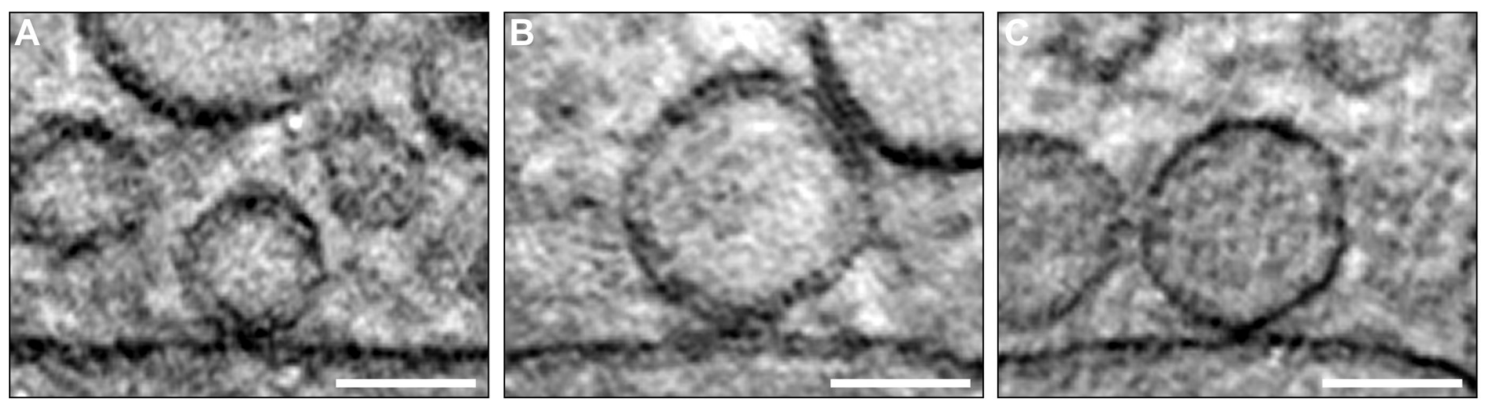

Figure 13. Three morphologically distinct vesicles dock at hippocampal mossy fiber synapses.

(A-C) Tomographic subvolumes of mossy fiber synapses showing docked synaptic vesicles (A), giant vesicles (B; clear-core vesicles with a diameter greater than $60 \mathrm{~nm}$ ), and DCVs (C) Scale bars: $50 \mathrm{~nm}$, A-C.

The second aim of my study was to perform a systematic analysis of the morphological nature of vesicles with respect to their subsynaptic distribution within Schaffer collateral and mossy fiber synapses. I was primarily motivated in this aim by the unexpected finding that mossy fiber active zones harbor a remarkable heterogeneity in vesicle size and morphological features. Of particular importance, my data reveal that docked vesicle pools at mossy fiber active zones comprise three morphological classes: 1) small, clear-cored synaptic vesicles with diameters ranging from 38 to $59 \mathrm{~nm}$ (Figure $13 \mathrm{~A}$ ); 2) large diameter clear-core vesicles ("giant vesicles") with diameters ranging from 60 to $120 \mathrm{~nm}$ (Figure $13 \mathrm{~B}$ ); and 3) DCVs with diameters ranging from 46 to $90 \mathrm{~nm}$ (Figure $13 \mathrm{C}$ ). In this section, I quantitate this heterogeneity and in particular, dissect ultrastructure-function relationships in hippocampal mossy fibers by relating the size and nature of morphologically distinct vesicle pools observed by electron tomography with functional pool measurements obtained via direct presynaptic capacitance recordings (Hallermann et al., 2003; Midorikawa and Sakaba, 2017).

\subsubsection{Large, clear-core vesicles dock exclusively at mossy fiber active zones in hippocampal slice cultures}

In striking contrast to Schaffer collateral synapses, mossy fiber synapses harbored an abundance of large diameter vesicles within reconstructed tomograms acquired from hippocampal slice cultures at DIV14 (Figure 14 A-D; Figure 23 A and B) and DIV28 (Figure 14 $\mathrm{E}-\mathrm{H}$; Figure $23 \mathrm{D}$ and C). Large diameter $(\varnothing>60 \mathrm{~nm})$ vesicles were only very rarely observed in 
Schaffer collaterals and were never detected within $60 \mathrm{~nm}$ of the active zone membrane (Figure $14 \mathrm{~A}, \mathrm{E}, \mathrm{I}$ ). At both developmental time points, giant vesicles accumulated in proximity to mossy fiber active zone release sites and dock at the presynaptic membrane (Figure $14 \mathrm{~A}$, E). Consequently, the mean diameter of vesicles docked at mossy fiber active zones was significantly larger than for those docked at Schaffer collaterals at DIV14 (Figure 14 B; 43.32 $\pm 0.354 \mathrm{~nm}, \mathrm{SC} ; 53.96 \pm 1.859 \mathrm{~nm}, \mathrm{MF} ; \mathrm{p}<0.001$ ) and DIV28 (Figure $14 \mathrm{~F} ; 44.37 \pm 0.230 \mathrm{~nm}$, SC; $49.40 \pm 1.034 \mathrm{~nm}, \mathrm{MF} ; \mathrm{p}<0.001$ ). Giant vesicles comprised approximately $22 \%$ and $12 \%$ of the docked vesicle pool at mossy fiber active zones at DIV14 (Figure 14 C; $0 \pm 0$, SC; $22.47 \pm$ 5.635\%, MF; $p<0.001$ ) and DIV28 (Figure 14 G; $0 \pm 0$, SC; $11.67 \pm 4.251 \%$, MF; $p<0.001$ ), respectively. Even when excluding all giant vesicles, the average diameter of docked synaptic vesicles $(\varnothing<60 \mathrm{~nm})$ in mossy fiber synapses was larger than in Schaffer collateral synapses (DIV14; Table 16; $43.32 \pm 0.35$ nm, SC; $46.78 \pm 0.46$ nm, MF; $p<0.001 ;$ DIV28; Table 18; 44.37 $\pm 0.23 \mathrm{~nm}, \mathrm{SC} ; 45.77 \pm 0.37 \mathrm{~nm}, \mathrm{MF} ; \mathrm{p}<0.001$ ). The abundance of giant vesicles within $40 \mathrm{~nm}$ of mossy fiber active zones was also slightly reduced from DIV14 (Figure 14 D, $0.355 \pm 0.055$, MF) to DIV28 (Figure $14 \mathrm{H}, 0.248 \pm 0.055, \mathrm{MF}$ ). This change in the proportion of docked giant vesicles is likely due to an increase in the number of docked synaptic vesicles rather than a decrease in the number of giant vesicles (Figure 14 G, Table 18, DIV28; Figure 9 C, Table 16, DIV14).

My findings provide the first clear evidence that giant vesicles dock in physical contact with the active zone membrane and comprise a substantial proportion of the docked vesicle pool in mossy fiber synapses.

\subsubsection{Mossy fiber giant vesicles are not a consequence of the slice culture procedure}

To investigate the possibility that giant vesicles observed in mossy fiber synapses in organotypic hippocampal slices represent structural artifacts resulting from the slice culture procedure, I performed a comparative analysis of Schaffer collateral and mossy fiber vesicle organization in acute slices prepared from P18 wild-type mice (Figure $14 \mathrm{I}-\mathrm{L}$ ). Consistent with my observations in cultured slices, giant vesicles were observed almost exclusively in mossy fiber synapses (Figure 14 I; Figure $23 \mathrm{E}$ and F). Although giant vesicles were less abundant in tomograms from acute slices (Table 19) compared to age-matched cultured slices (Table 15), they still exhibited a clear tendency to accumulate in proximity to, and to dock at, mossy fiber 
active zone release sites (Figure 14 I). As in cultured slices (Figure 14 B, F), the mean diameter of docked vesicles in acute slices was larger in mossy fiber synapses compared to Schaffer collaterals (Figure $14 \mathrm{~J} ; 45.59 \pm 0.318 \mathrm{~nm}, \mathrm{SC} ; 50.67 \pm 0.842 \mathrm{~nm}, \mathrm{MF} ; \mathrm{p}<0.001$ ). Giant vesicles comprised approximately $5 \%$ of the total docked vesicle population at mossy fiber active zones (Figure $14 \mathrm{~K} ; 5.366 \pm 1.595 \%$, MF). Only one Schaffer collateral synaptic profile contained a docked giant vesicle. The majority of giant vesicles observed in mossy fiber synaptic profiles from acute slices accumulated within $40 \mathrm{~nm}$ of the active zone (Figure $14 \mathrm{~L}$, acute slice, $0.012 \pm 0.12$, SC; $0.122 \pm 0.024$, MF; $p=0.001$ ).

These results indicate that giant vesicles are also a distinguishing ultrastructural feature of mossy fiber synapses in vivo and are capable of docking at the active zone membrane. Thus, giant vesicles are not merely an artifact of the organotypic slice culture procedure. Although giant vesicles appeared less abundant in acute compared to cultured slices, the potential consequences of mechanical trauma and anoxia induced during acute slice preparation on vesicle organization must be taken into consideration when interpreting this result. 
A

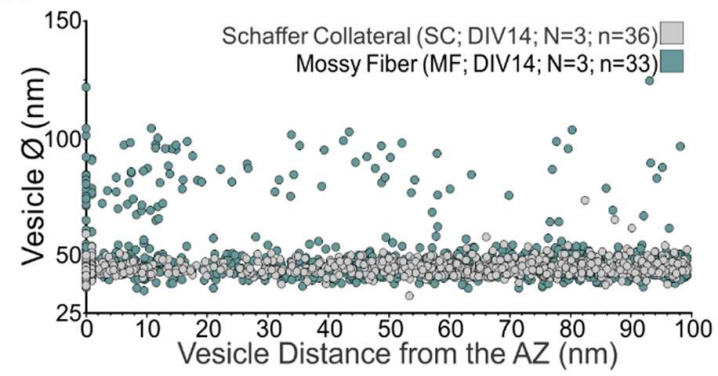

E

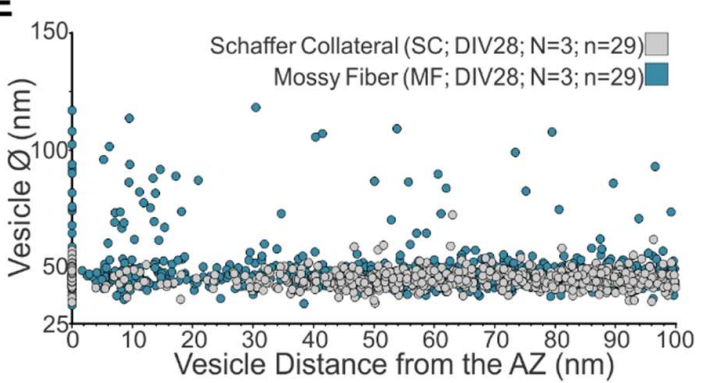

I

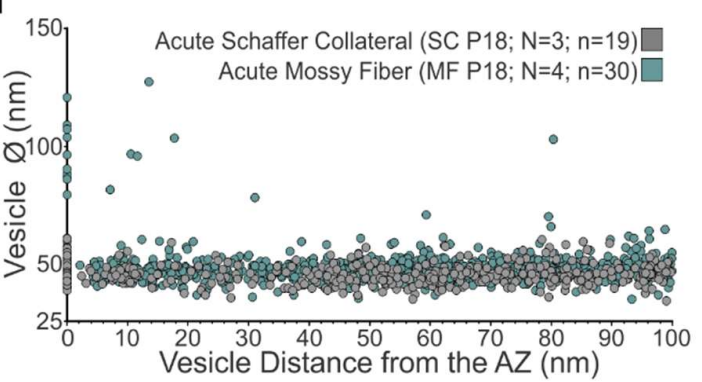

M

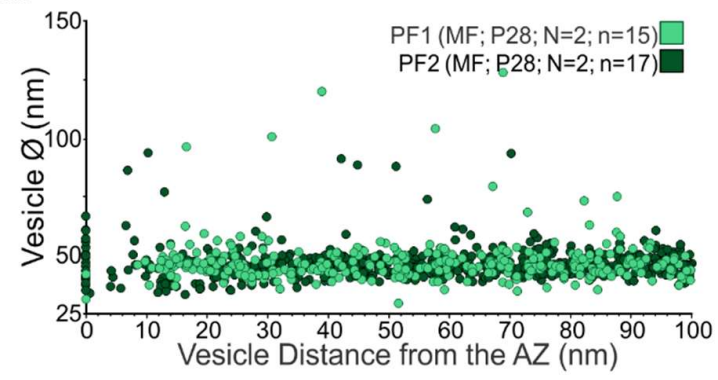

B

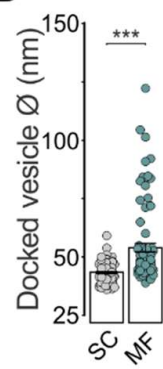

F

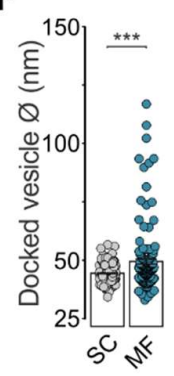

$\mathbf{J}$

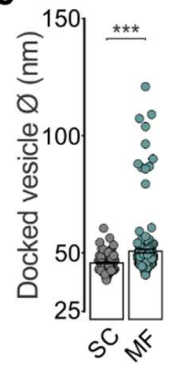

N

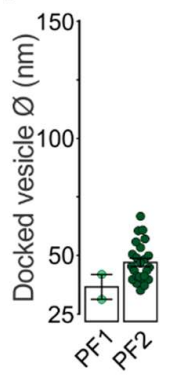

C

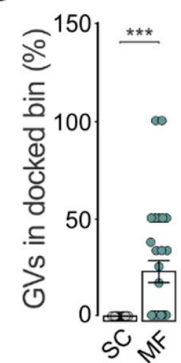

G

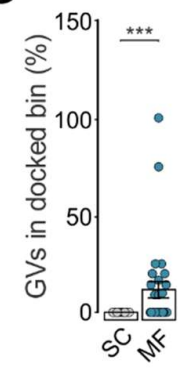

K

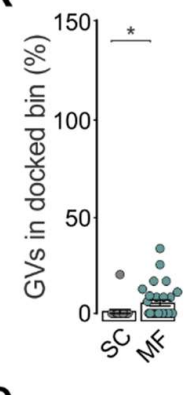

0

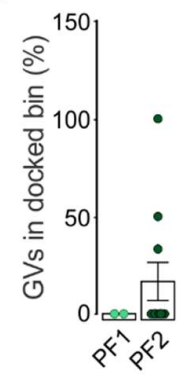

D

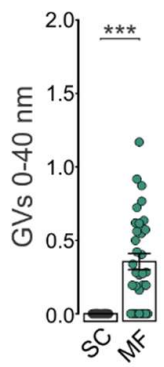

H

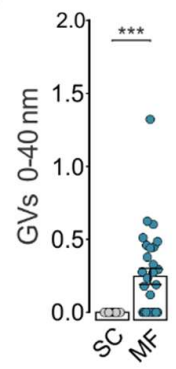

L

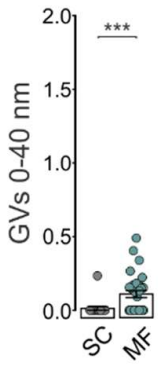

P

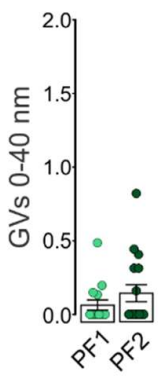

Figure 14. Distribution of giant vesicles in mossy fiber synapses from slice cultures, acute slice preparations, and perfusion-fixed hippocampal tissue.

(A-H) Analysis of Schaffer collateral and mossy fiber synapses from hippocampal slice cultures at DIV14 (AD) and DIV28 (E-H). (I-L) Analysis of Schaffer collateral and mossy fiber synapses from acute slice preparations from P18 wild-type mice. (M-P) Analysis of mossy fiber synapses from transcardially perfused P28 wild-type mice with two different aldehyde cocktails: PF1 (ice-cold 4\% PFA, 2.5\% GA in $0.1 \mathrm{M} \mathrm{PB})$, or PF2 $\left(37^{\circ} \mathrm{C}, 2 \%\right.$ PFA, $2.5 \% \mathrm{GA}, 2 \mathrm{mM} \mathrm{CaCl}_{2}$ in $0.1 \mathrm{M}$ cacodylate buffer. (A, E, I, M) Clear-core vesicle diameters and their respective distance to the active zone. (B, F, J, N) Scatter plots of docked vesicle diameters. (C, G, K, O) Scatter plots of the respective proportions of the docked vesicle pool occupied by giant vesicles. (D, H, L, P) Scatter plots of the number of giant vesicles within $40 \mathrm{~nm}$ of the active zone membrane normalized to active zone area. Statistical significance is represented as ${ }^{*}, \mathrm{p}<0.05 ; *, \mathrm{p}<0.005 ; * * *, \mathrm{p}<0.001$. $\mathrm{N}=$ number of cultures DIV14 (A-D) and DIV28 (E-H) and number of animals used for acute slice (I-L) and perfusion fixation preparations (M-P); $\mathbf{n}=$ number of active zones. See Tables 15-22 for full statistical analyses. 


\subsubsection{The organization of giant vesicles at mossy fiber active zones is sensitive to aldehyde fixation}

In further investigation of ex vivo preparations, giant vesicles were observed in mossy fiberCA3 synapses in wild-type animals perfusion-fixed with either PF1 (ice-cold 4\% PFA, 2.5\% GA in $0.1 \mathrm{M} \mathrm{PB})$, or PF2 $\left(37^{\circ} \mathrm{C}, 2 \% \mathrm{PFA}, 2.5 \% \mathrm{GA}, 2 \mathrm{mM} \mathrm{CaCl}_{2}\right.$ in $0.1 \mathrm{M}$ cacodylate buffer) (Figure $14 \mathrm{M}$; Figure $25 \mathrm{~A}$ and $\mathrm{B}$ ). The effects of PF1 were profound; only two docked synaptic vesicles were found in 15 tomograms analyzed (Figure $14 \mathrm{~N} ; 36.67 \pm 5.284 \mathrm{~nm}, \mathrm{PF} 1 ; 47.16 \pm 1.711 \mathrm{~nm}$, PF2; Figure $25 \mathrm{~A}$ and B). Giant vesicles were docked at the active zone membrane in mossy fiber synapses from perfusion fixed animals (Figure $140 ; 0 \pm 0$, PF1; $16.67 \pm 9.796 \%, P F 2$ ), a second ex vivo preparation harboring docked giant vesicles. While relatively low in abundance, giant vesicles were present in mossy fiber synapses from both perfusion fixation protocols within $40 \mathrm{~nm}$ (Figure 14 P; $0.065 \pm 0.035$, PF1; $0.145 \pm 0.058, \mathrm{PF} 2 ; \mathrm{p}=0.45$ ) and 100 $\mathrm{nm}$ of the active zone (Table $21 ; 0.205 \pm 0.073, \mathrm{PF} 1 ; 0.263 \pm 0.076, \mathrm{PF} 2 ; \mathrm{p}=0.069$ ).

In summary, these data indicate that giant vesicles are not an artifact of slice culture preparations, and this finding is supported by several ex vivo preparations. Similar to the consequences of perfusion fixation on synaptic vesicle pools, perfusion fixation depletes docked and membrane-proximal pools of giant vesicles at mossy fiber active zones. However, the potential effects of the perfusion procedure itself (i.e. anoxia) and the lower number of giant vesicles observed within the volume of presynaptic terminals of mossy fibers in ex vivo compared to in vitro preparations must also be considered when interpreting these results.

\subsubsection{Acute pharmacological blockade of network activity does not alter giant vesicle organization}

To examine the possibility that spontaneous slice activity contributes to the formation and presynaptic organization of giant vesicles in hippocampal mossy fibers, I treated wild-type slice cultures at DIV14 with drug cocktails designed to pharmacologically silence network activity for 10 minutes prior to HPF. Three conditions were analyzed in this experiment: 1 ) T/N/A: TTX, to block sodium-propagated action potentials, supplemented with either NBQX and D-AP5 to block AMPA and NMDA receptor blockers, respectively; 2) T/D: TTX with DCGIV, an mGluR2 receptor agonist that specifically blocks synaptic transmission in mossy fiber synapses (Kamiya and Ozawa, 1999); and 3) VC, comprised of slice culture medium applied in the same manner as the pharmacologically treated slices prior to HPF. Giant vesicles exhibited 
a similar spatial distribution within $100 \mathrm{~nm}$ of mossy fiber active zones after T/N/A and T/D treatment compared to VC (Figure $15 \mathrm{~A}$; Figure $24 \mathrm{~A}$ and $\mathrm{B}$ ). Although the mean diameter of docked vesicles was unchanged after T/N/A and T/D treatment when compared to that of VC mossy fiber synapses (Figure 15 B, $48.84 \pm 0.884 \mathrm{~nm}, \mathrm{VC} ; 49.29 \pm 0.517 \mathrm{~nm}, \mathrm{~T} / \mathrm{N} / \mathrm{A} ; 53.86 \pm$ $1.568 \mathrm{~nm}, T / D ; p=0.10$ ), the proportion of giant vesicles comprising the total docked vesicle population was significantly greater after mossy fiber transmission was inhibited by $T / D$ application when compared to T/N/A (Figure 15 C; $6.944 \pm 3.943 \%$, VC; $0.926 \pm 0.926 \%$, $T / N / A ; 15.64 \pm 4.523 \%, T / D ; p=0.005)$. Despite an increase in giant vesicle accumulation within $20 \mathrm{~nm}$ of mossy fiber active zones after T/D treatment (Table 25; $0.151 \pm 0.047$, VC; $0.080 \pm 0.031, T / N / A ; 0.215 \pm 0.063, T / D ; p=0.05)$, the abundance of giant vesicles within $40 \mathrm{~nm}$ was not significantly different from VC and T/N/A treatments (Figure 15 D; $0.344 \pm$ $0.080, V C ; 0.383 \pm 0.063, T / N / A ; 0.538 \pm 0.088, T / D ; p=0.17$ ), indicating giant vesicles are organized closer to the plasma membrane after application of DCG-IV.

These results demonstrate that the relative abundance and spatial distribution of giant vesicles in mossy fiber synapses is largely unaffected by acute pharmacological blockade of network activity in cultured slices. Mossy fiber giant vesicles are therefore unlikely to represent structural endocytic intermediates formed by excessive spontaneous activity in cultured hippocampal slices.

\subsubsection{Giant vesicles are present, but do not dock in Munc13-deficient mossy fiber synapses}

To more stringently investigate the possibility that mossy fiber giant vesicles represent structural intermediates generated by activity-dependent endocytic mechanisms, I analyzed the spatial organization of giant vesicles in mossy fiber synapses from Munc13-deficient slices. Munc13-deficient synapses exhibit an almost complete loss of spontaneous and evoked synaptic transmission (Augustin et al., 1999; Sigler et al., 2017; Varoqueaux et al., 2002). However, under culture conditions, neurons from Munc13-deficient slices develop normally 
A

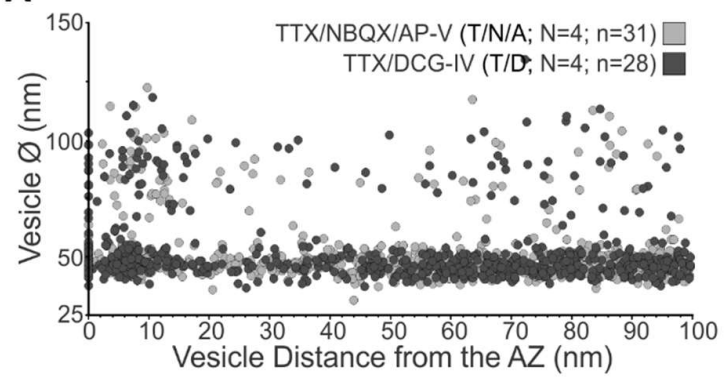

E

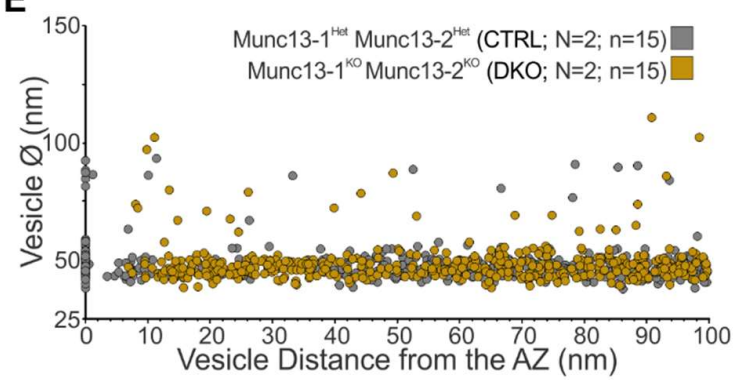

I

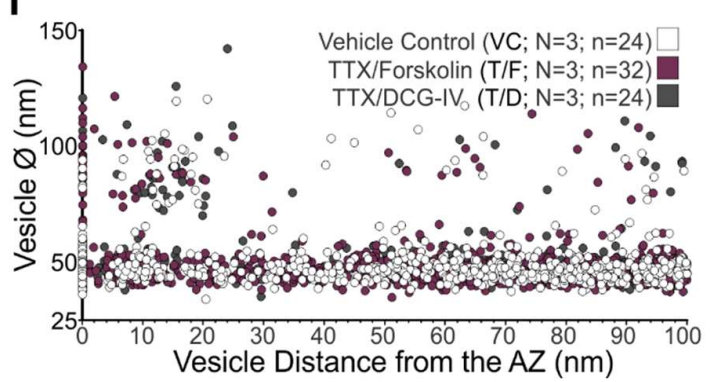

B

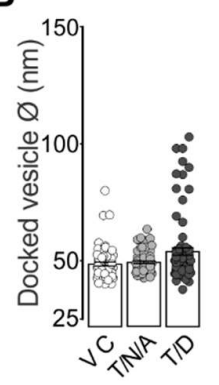

F

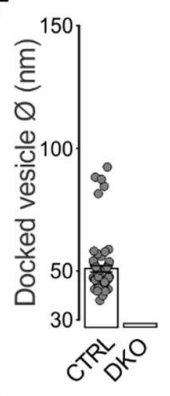

\section{J}

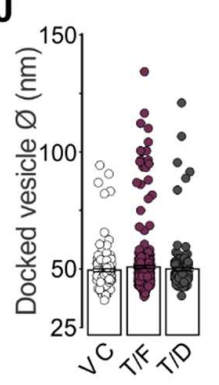

C

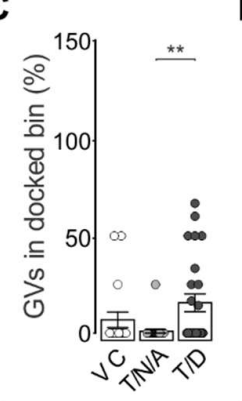

G

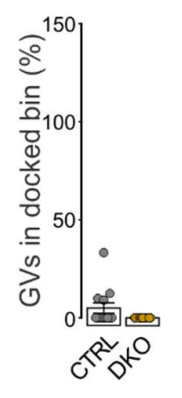

K

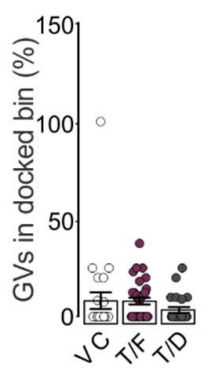

D

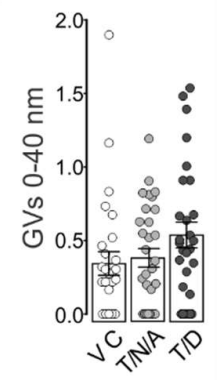

H

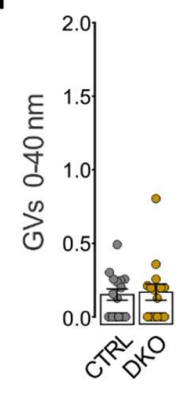

L

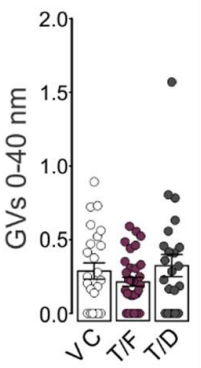

Figure 15. Distribution of giant vesicles in mossy fiber synapses from slice cultures after acute silencing, genetic silencing, or after pharmacological manipulation of release probability.

(A-D) Analysis of mossy fiber synapses after a 10 minute pharmacological treatment of slice cultures at DIV14 with one of three conditions: 1) T/N/A: TTX, to block sodium-propagated action potentials, supplemented with either NBQX and D-AP5 to block AMPA and NMDA receptor blockers, respectively; 2) T/D: TTX with DCG-IV, an mGluR2 receptor agonist that specifically blocks synaptic transmission in mossy fiber synapses (Kamiya and Ozawa, 1999); and 3) VC: a vehicle control comprised of slice culture medium. (E-H) Analysis of mossy fiber synapses from Munc13-1/2 DKO and CTRL slice cultures at DIV28. (I-L) Analysis of mossy fiber synapses after 15 minute treatment with either: 1) T/F: TTX, to block sodium propagated action potentials, and forskolin, an activator of AC1 which increases presynaptic CAMP concentrations (Chavez-Noriega and Stevens, 1994; Dixon and Atwood, 1989; Seamon et al., 1983) thus increasing synaptic transmission (Evans and Morgan, 2003; Seino and Shibasaki, 2005; Weisskopf et al., 1994); 2) T/D: TTX with DCG-IV, and mGluR2 receptor agonist that specifically blocks synaptic transmission in mossy fiber synapses (Kamiya and Ozawa, 1999); and 3) VC: a vehicle control comprised of TTX in slice culture medium. (A, E, I) Clear-core vesicle diameters and their respective distance to the active zone membrane. (B, F, J) Scatter plots of docked vesicle diameters. (C, G, K) Scatter plots of the respective proportions of the docked vesicle pool occupied by giant vesicles. $(D, H, L)$ Scatter plots of the number of giant vesicles within $40 \mathrm{~nm}$ of the active zone membrane normalized to active zone area. Statistical significance is represented as *, $\mathrm{p}<0.05 ;{ }^{* *}, \mathrm{p}<0.005 ;{ }^{* * *}, \mathrm{p}<0.001$. $\mathrm{N}=$ number of cultures; $\mathrm{n}=$ number of active zones. See Tables $23-28$ for full statistical analyses.

and establish synaptic connections within anatomically intact circuits. Thus, an ultrastructural 
examination of mossy fiber synapses in Munc13-deficient slices offers an opportunity to characterize the spatial organization of vesicles in a synapse that has been genetically silenced since "birth" and as such, has never actively participated in synaptic transmission.

Mossy fiber giant vesicles were observed in tomograms of both control and Munc13-1/2 DKO slices (Figure $15 \mathrm{E}$; Figure $24 \mathrm{C}$ and D). However, no vesicles of any type docked at mossy fiber active zones in Munc13-1/2 DKO slices (Figure $11 \mathrm{E}, \mathrm{F}, \mathrm{G} ; 0.051 \pm 0.024, \mathrm{CTRL} ; 0 \pm 0$, DKO; $\mathrm{p}=0.10)$. Instead, vesicles appeared to accumulate at approximately $10 \mathrm{~nm}$ from the active zone in mossy fibers from Munc13-1/2 DKO slices (Figure $11 \mathrm{E}$ ). Despite this striking docking deficit, the densities of giant vesicles within $40 \mathrm{~nm}$ (Figure $15 \mathrm{H} ; 0.152 \pm 0.039, \mathrm{CTRL} ; 0.169 \pm$ 0.055, DKO; $p=0.86$ ) and $100 \mathrm{~nm}$ (Table 23; $0.229 \pm 0.058, C T R L ; 0.315 \pm 0.080, D K O ; p=0.39$ ) of the active zone membrane were highly comparable between Munc13-1/2 CTRL and DKO mossy fiber synapses.

The results of these experiments effectively exclude the possibility that mossy fiber giant vesicles are formed via activity-dependent forms of compensatory endocytosis (Delvendahl et al., 2016; Watanabe et al., 2013a). My findings demonstrate that like synaptic vesicles, mossy fiber giant vesicles are dependent on Munc13 priming proteins to dock at the active zone membrane. This discovery raises several important questions: Do giant vesicles fuse at active zone release sites? Do giant vesicles contain neurotransmitter and contribute to glutamatergic signaling at mossy fiber synapses?

\subsubsection{Mossy fiber giant vesicles are the morphological correlate of giant mEPSCs recorded in CA3 pyramidal neurons}

Although my data provide the first unequivocal evidence that giant vesicles dock in physical contact with mossy fiber active zones, their existence has been previously reported (Figure 16 A) (Borges-Merjane et al., 2020; Henze et al., 2002b; Laatsch and Cowan, 1966; Rollenhagen et al., 2007). These observations became of particular importance upon the demonstration that large amplitude (giant) mEPSCs can be recorded from CA3 pyramidal neurons, and that the giant mEPSCs are monoquantal and of mossy fiber origin (Henze et al., $1997,2002 b)$. Based on the assumption that giant vesicles contain neurotransmitter and are capable of fusing at mossy fiber active zones to generate giant mEPSCs, I rationalized that the 
relative distribution of mEPSC amplitudes should correlate with the relative proportion of docked giant vesicles visualized and quantified in electron tomograms. To this end, I collaborated with Dr. Bekir Altas, who used patch-clamp electrophysiology to isolate and record mEPSCs from CA3 pyramidal cells in cultured hippocampal slices at DIV14 (Figure 16 B). Initial baselines of all mEPSC events were recorded for a 5-minute period in the presence of TTX, to block sodium-propagated action potentials, and the GABA receptor blocker bicuculline, to exclude contributions from GABAergic transmission (Figure 16 B; black trace). Subsequently, DCG-IV was applied to the bath solution and all DCG-IV-insensitive mEPSCS were recorded for the next 5-minute period (Figure $16 \mathrm{~B}$; gray trace; 5-minute epoch was between the $10^{\text {th }}$ and $15^{\text {th }}$ minute after DCG-IV wash-on). Inhibition of mossy fiber synaptic transmission with DCG-IV reduced the frequency of mEPSC events regardless of amplitude (Figure $16 \mathrm{C}$ ). To test whether docked giant vesicles are neurotransmitter-filled, I next compared the frequency of giant mEPSCs to the proportion of docked giant vesicles. To correlate the proportion of giant mEPSC events with the relative proportion of docked giant vesicles at mossy fiber active zones, I subtracted the amplitudes of DCG-IV-insensitive events from the baseline mEPSCs to get an estimate of the DCG-IV-sensitive amplitudes (Figure 16 D, purple line). I then rationalized that the most frequently observed DCG-IV-sensitive mEPSC amplitudes (statistical mode of DCG-IV-sensitive mEPSC amplitudes $=10 \mathrm{pA}$ ) reflected fusion and transmitter release from docked vesicles with the most frequently observed dimensions (the statistical mode of docked synaptic vesicle diameters $=44 \mathrm{~nm}$ ). I calculated the inner lumenal volume of a synaptic vesicle with an outer diameter of $44 \mathrm{~nm}$, accounting for the lipid bilayer ( $\sim 4 \mathrm{~nm}$ of radius measured from tomograms; $\sim 24,400 \mathrm{~nm}^{3}$ lumenal volume). I then calculated the theoretical mEPSC amplitude that would arise from the fusion of a vesicle at the lower threshold for classification as a giant vesicle $(\varnothing=60 \mathrm{~nm}$; 30 pA; Figure $16 \mathrm{D}$ dotted line). Approximately $27 \%$ of DCG-IV-sensitive mEPSCs were larger than $30 \mathrm{pA}$, in agreement with my finding that approximately $20 \%$ of all docked vesicles are giant vesicles in agematched mossy fiber synapses (Figure $16 \mathrm{C}$ ). It is important to note that my calculations are based on several assumptions: i) DCG-IV-sensitive mEPSC events originated from docked 


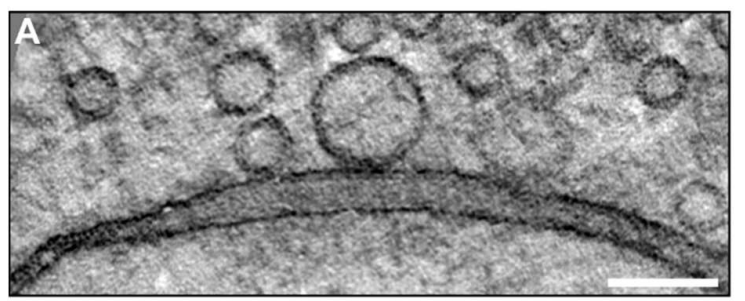

B

C

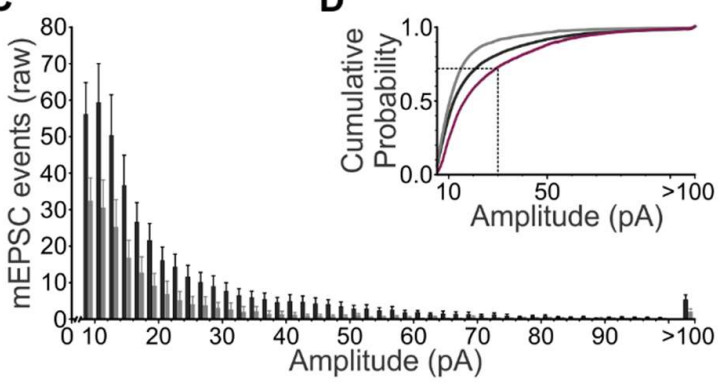

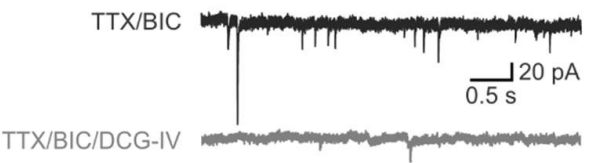

$\mathbf{E}$

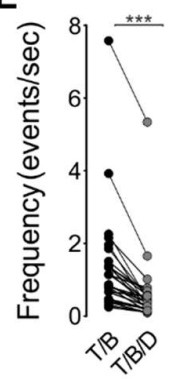

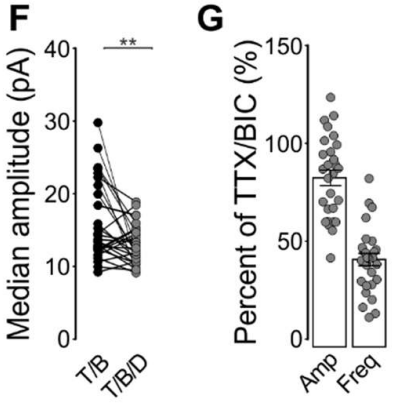

Figure 16. Electrophysiological and morphological analysis of giant vesicles in mossy fiber synapses.

(A) Tomographic subvolume of a mossy fiber synapse with a giant vesicle docked directly at the active zone membrane. (B-G) Effects of DCG-IV on mEPSC events recorded in CA3 pyramidal neurons in hippocampal slice cultures at DIV14. (B) Example traces of mEPSCs recorded from CA3 pyramidal neurons in the presence of $1 \mu \mathrm{M} T \mathrm{TX}$ and $10 \mu \mathrm{M}$ bicuculline (BIC) before (black trace) and after the wash on of $2 \mu \mathrm{M}$ DCG-IV (grey trace). (C) Frequency distribution of mEPSC amplitudes recorded in CA3 pyramidal neurons before (black) and after (gray) application of DCG-IV (2 pA bins from 8 pA until $100 \mathrm{pA}$ and all events greater than $100 \mathrm{pA}$ are pooled in one bin). (D) Cumulative distribution of mEPSC amplitude before and after application of DCGIV. (E) Before-after scatter plot of mEPSC frequency before (T/B) and after (T/B/D) application DCG-IV. (F) Before-after scatter plot of median mEPSC amplitude before and after the application of DCG-IV. (G) Relative changes in the median amplitude and frequency after the application of DCG-IV normalized to TTX/BIC. Statistical significance is represented as ${ }^{*}, \mathrm{p}<0.05 ;{ }^{* *}, \mathrm{p}<0.005 ;{ }^{* * *}, \mathrm{p}<0.001$. $\mathrm{N}=2$ cultures; $\mathrm{n}=28$ cells. Scale bar: $100 \mathrm{~nm}$, A. See Table 29 for full statistical analysis.

synaptic vesicles at mossy fiber synapses; ii) synaptic vesicle filling was proportional to the size of a given vesicle (Bruns et al., 2000); and iii) postsynaptic receptor saturation was negligible.

The amplitudes of the remaining DCG-IV-insensitive mEPSCs were reduced compared to amplitudes of all mEPSCs (Figure $16 \mathrm{D}$ ). DCG-IV caused a significant reduction in the mEPSC frequency in CA3 pyramidal neurons compared to all mEPSC events before the application of DCG-IV (Figure $16 \mathrm{E} ; 1.376 \pm 0.273$ events/sec, TTX/BIC; $0.60 \pm 0.186$ events/sec, TTX/BIC/DCG-IV; $p<0.001)$. The median amplitude of mEPSC events recorded in CA3 pyramidal neurons was significantly reduced after DCG-IV application (Figure 16 F; $16.25 \pm$ $1.031 \mathrm{pA}, \mathrm{TTX} / \mathrm{BIC} ; 13.0 \pm 0.524 \mathrm{pA}, \mathrm{TTX} / \mathrm{BIC} / \mathrm{DCG}-\mathrm{IV} ; \mathrm{p}=0.007)$. The specific inhibition of mossy fiber synaptic transmission with DCG-IV demonstrates that $41 \%$ of mEPSCs onto CA3 
pyramidal neurons are insensitive to DCG-IV (Figure 16 G; $40.75 \pm 3.20 \%$ ), and likely arise from excitatory collaterals from other CA3 pyramidal neurons described to form in rat slice cultures (Frotscher and Gähwiler, 1988). Furthermore, the median amplitude of DCG-IV-insensitive events was reduced to about $82 \%$ of all mEPSC events measured prior to the application of DCG-IV (Figure $16 \mathrm{G} ; 82.43 \pm 3.939 \%$ ), meaning that many but not all giant mEPSC events were sensitive to DCG-IV. It is unclear the extent at which DCG-IV inhibits spontaneous vesicle fusion at hippocampal mossy fiber synapses however these results are in agreement with the reduction in mEPSC events after gamma-irradiation of hippocampal granule cells (Henze et al., 1997).

My findings indicate a close correlation between the relative proportions of DCG-IV-sensitive giant mEPSCs and docked giant vesicles at mossy fiber synapses, thereby supporting the hypothesis that giant vesicles contribute to glutamatergic signaling at mossy fiber-CA3 synapses. A potential contribution of multivesicular release events to the larger amplitude mEPSCs recorded in CA3 pyramidal neurons cannot be completely excluded. My data support the notion that giant vesicles indeed contain neurotransmitter and that they have the potential to profoundly influence synaptic transmission at mossy fiber-CA3 synapses (Henze et al., 2002b). I therefore investigated whether changes in synaptic strength correlate with corresponding changes in the numbers of docked, and presumably fusion-competent, giant vesicles.

\subsubsection{Pharmacological manipulation of presynaptic CAMP does not alter giant vesicle organization}

If giant vesicles represent bona fide synaptic vesicles with the capacity to release large quanta of neurotransmitter, I rationalized that alterations in their availability at active zone release sites may contribute to changes in mossy fiber synaptic transmission efficacy upon manipulation of presynaptic CAMP levels. I treated wild-type slice cultures at DIV28 with drug cocktails to either increase or decrease presynaptic CAMP concentrations. Three conditions were analyzed in this experiment: 1) T/F: TTX, to block sodium propagated action potentials, and forskolin, an activator of AC1 which increases presynaptic CAMP concentrations (Chavez-

Noriega and Stevens, 1994; Dixon and Atwood, 1989; Seamon et al., 1983) thus increasing synaptic transmission (Evans and Morgan, 2003; Seino and Shibasaki, 2005; Weisskopf et al., 1994); 2) T/D: TTX with DCG-IV, an mGluR2 receptor agonist that specifically blocks synaptic 
transmission in mossy fiber synapses (Kamiya and Ozawa, 1999); and 3) VC: comprised of TTX in slice culture medium. Consistent with data obtained from untreated mossy fiber synapses at DIV28, mossy fiber synapses harbored giant vesicles distributed throughout the synaptic subvolume following pharmacological manipulation of presynaptic cAMP levels (Figure 15 I; Figure $24 \mathrm{E}$ and F). Giant vesicles docked at mossy fiber synapses in VC, T/F, and T/D (Figure $15 \mathrm{~J} ; 49.26 \pm 0.631, \mathrm{VC} ; 50.69 \pm 0.662, \mathrm{~T} / \mathrm{F} ; 49.68 \pm 0.754, \mathrm{~T} / \mathrm{D} ; \mathrm{p}=0.94)$, however the proportion of docked giant vesicles per active zone was slightly lower when presynaptic levels of CAMP were reduced with DCG-IV, although this tendency did not reach statistical significance (Figure $15 \mathrm{~K} ; 8.241 \pm 4.179 \%$, VC; $8.012 \pm 1.677 \%$, T/F; $3.504 \pm 1.420 \%$, T/D; $p=0.09$ ). The densities of giant vesicles were comparable regardless of presynaptic cAMP concentrations within $40 \mathrm{~nm}$ (Figure $15 \mathrm{~L} ; 0.288 \pm 0.055$, VC; $0.215 \pm 0.030, \mathrm{~T} / \mathrm{F} ; 0.325 \pm 0.075$, $T / D ; p=0.62$ ) and within $100 \mathrm{~nm}$ of the active zone (Table 27; $0.561 \pm 0.098, \mathrm{VC} ; 0.333 \pm 0.44$, $T / F ; 0.476 \pm 0.093, T / D ; p=0.93)$.

These data demonstrate that the organization of giant vesicles at mossy fiber active zones is insensitive to acute, pharmacologically induced changes in presynaptic cAMP concentrations. Therefore, forskolin-induced enhancement of mossy fiber transmitter release efficacy is unlikely to derive from increased availability or fusion of giant vesicles at mossy fiber active zones.

\subsubsection{Giant vesicles may originate in granule cell somas and are not restricted to immature mossy fiber synapses}

Although the existence of giant vesicles in mossy fiber synapses is well-documented (Henze et al., 2002b; Laatsch and Cowan, 1966; Rollenhagen et al., 2007), the origin of this vesicle type in mossy fiber synapses is unknown. While it is possible that giant vesicles are precursor vesicles that traffic from granule cell somata, the molecular identity of giant vesicles has yet to be characterized. To this end, I took low magnification electron tomograms of mossy fiber axonal projections from granule cells in acute hippocampal slices. All vesicle types docked at mossy fiber active zones were observed in electron tomograms of mossy fiber axonal projections from granule cells to the CA3 in acute hippocampal slices from P18 wild-type mice (Figure $17 \mathrm{~A}-\mathrm{I})$. These vesicles include synaptic vesicles (Figure $17 \mathrm{~A}$; open arrowheads), giant vesicles (Figure 17 A, B, E, F, G, H, I; grey arrowheads), and DCVs (Figure 17 A, C, D, H). Giant vesicles in the mossy fiber axons are positioned within nanometers of axonal microtubules 

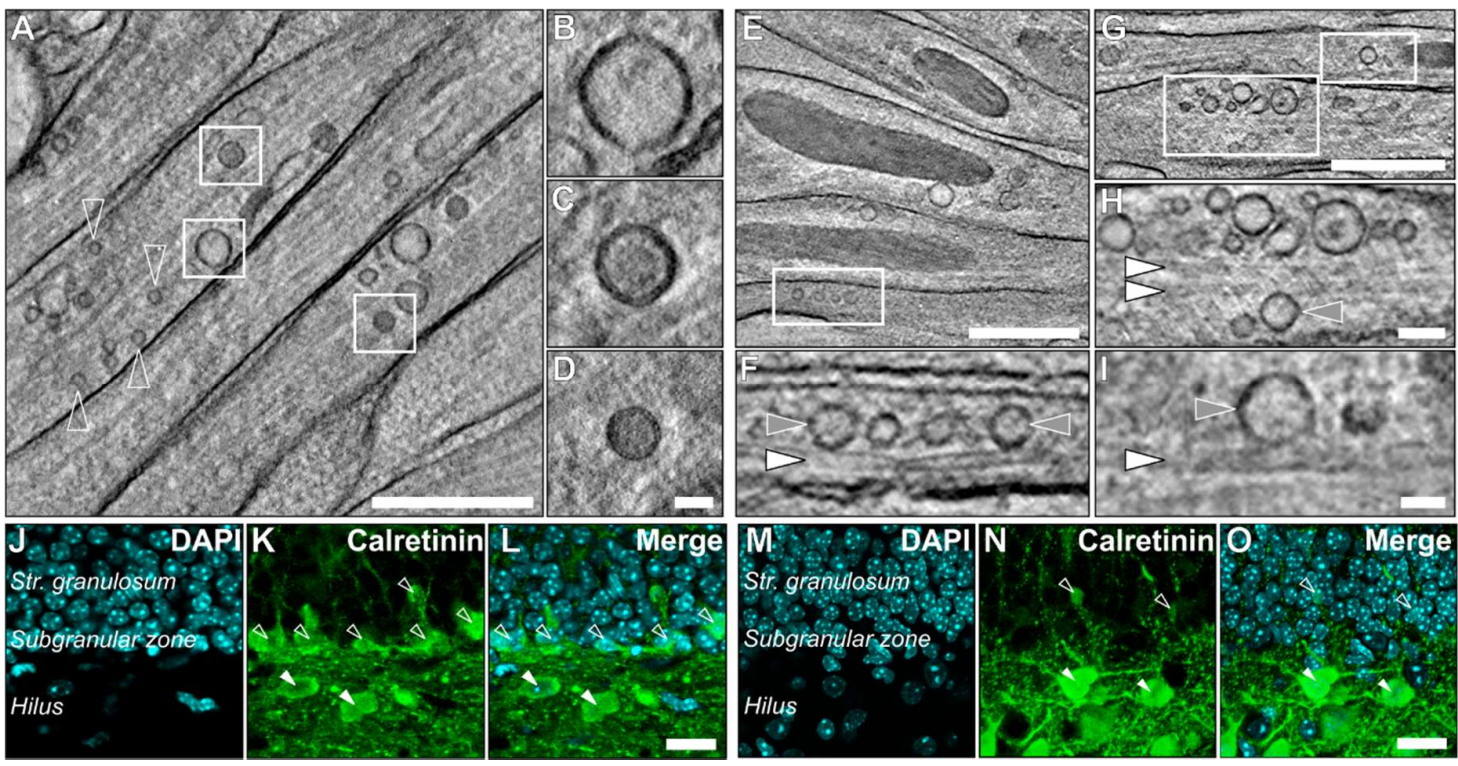

Figure 17. Giant vesicles may have somatic origins and are not merely an early developmental phenomenon.

(A-I) Tomographic subvolume of low-magnification electron tomograms taken of mossy fiber axonal projections from acute slice preparations of P18 wild-type mice. All three vesicle types that are observed docked at mossy fiber active zones, synaptic vesicles (open arrowheads), giant vesicles (B), and DCVs (C-D) Positioning of synaptic vesicles, giant vesicles, and DCVs in proximity to axonal microtubules (E-I) indicate trafficking of these vesicles within the granule cell axons. Giant vesicles indicated with gray arrowheads, microtubules with white arrowheads $(\mathbf{F}, \mathbf{H}-\mathbf{I})$. (J-O) Confocal images of the subgranular zone in the dentate gyrus from acute hippocampal slices (J-L) and slice cultures at DIV28 (M-O) where calretinin-expressing (K and $\mathbf{N}$; green) new born granule cells are observed in the acute slice (K; open arrowheads), but rarely in the slice culture ( $\mathbf{N})$. ( $\mathbf{J}$ and $\mathbf{M}$; visualization of nuclei with DAPI, cyan). Calretinin-positive hilar mossy cells (K-L; $\mathbf{N}-\mathbf{O}$; white arrowheads) are unchanged in slice cultures (N-O) compared to acute slices (K-L). (L, O) Merged confocal images of DAPI and calretinin labeling in acute slices (L) and slice culture (0). Scale Bars: $500 \mathrm{~nm}, \mathbf{A}$, E, G; 100 nm, H; 50 nm, B-D, P $20 \mu \mathrm{m}, \mathbf{J}-\mathbf{O}$.

(Figure $17 \mathrm{~F}, \mathrm{H}, \mathrm{l}$; white arrowheads), indicative of anterograde trafficking to the synaptic bouton. This evidence, however, arises from images of fixed tissues. Additional experimentation is necessary to determine the direction of the axonal giant vesicles.

Neurogenesis in the hippocampal DG continues well into adulthood in rodents (Mongiat and Schinder, 2011). To exclude the possibility that giant vesicles were present in immature mossy fiber boutons and thus an artifact of newly generated granule cells, I compared the number of newly generated granule neurons from ex vivo preparations and age-matched hippocampal slice cultures. Newly generated calretinin-positive granule cells (Brandt et al., 2003) found in the subgranular zone of the DG in acute brain slices (open arrowheads; Figure $17 \mathrm{~J}-\mathrm{L})$ were almost completely absent in hippocampal slice cultures (Figure 17M-O), indicating an almost complete cessation of granule cell neurogenesis in our model system. In 
cultured hippocampal tissue, calretinin immunoreactivity was restricted to hilar mossy cells (white arrowheads in Figure $17 \mathrm{~K}, \mathrm{~L}, \mathrm{~N}, \mathrm{O}$ ). These findings indicate that the analysis of mossy fiber synapses from hippocampal slice cultures did not unintentionally include immature mossy fiber boutons formed by newborn granule cells during the culture period, therefore demonstrating that giant vesicles are not an artifact of mossy fiber development. These results do not exclude the possibility that other mechanisms of giant vesicle formation may exist and indicate that further investigation is required. 


\subsection{DCVs dock at the active zone in mossy fiber synapses}

DCVs are vesicular organelles that transport and secrete peptide signaling molecules in many cells within a living organism. In the central nervous system, DCVs package, transport, and release neuropeptides that modulate the function of synaptic transmission on the pre- and postsynaptic cell (Salio et al., 2006). DCVs can be induced to fuse at both synaptic and extrasynaptic sites (van de Bospoort et al., 2012; Farina et al., 2015; Tao et al., 2018a), however, ultrastructural analyses have typically detected them at a distance from the active zone (van de Bospoort et al., 2012; Cifuentes et al., 2008; Imig et al., 2014). Consistent with the relatively high abundance of neuropeptides produced by dentate granule cells (Danzer and McNamara, 2004; Derrick et al., 1992; Salin et al., 1995; Simmons and Chavkin, 1996; Weisskopf et al., 1993), and with previous observations of DCVs within mossy fiber synapses in ex vivo preparations (Chicurel and Harris, 1992; Commons and Milner, 1996; Dieni et al., 2012, 2015; Rollenhagen et al., 2007; Sadakata et al., 2013), my data demonstrate that DCVs not only accumulate, but actually dock in physical contact with the active zone membrane.

\subsubsection{DCVs dock at mossy fiber active zones in slice cultures and acute slice preparations}

In mossy fiber synapses from hippocampal slice cultures at both DIV14 and DIV28, DCVs were observed within $100 \mathrm{~nm}$ of the active zone and had a tendency to accumulate within $20 \mathrm{~nm}$ of the active zone (Figure 18 A; DIV14 green, DIV28 grey). Similar numbers of DCVs docked at mossy fiber active zones at both developmental time points (Figure $18 \mathrm{~B} ; 0.075 \pm 0.023$, DIV14; $0.050 \pm 0.019$, DIV28). The majority of DCVs measured were located within $40 \mathrm{~nm}$ of the active zone (Figure 18 C, $0.163 \pm 0.036$, DIV14; $0.190 \pm 0.032$, DIV28; compared to Figure $18 \mathrm{D}, 100 \mathrm{~nm}, 0.278 \pm 0.049$, DIV14; $0.282 \pm 0.047$, DIV28). In contrast, no DCVs were found within $100 \mathrm{~nm}$ of the active zone in Schaffer collateral synapses at either DIV14 or DIV28, although a number of DCVs were observed beyond the $100 \mathrm{~nm}$ cutoff (data not shown).

In acute slice preparations from P18 wild-type mice, DCVs were also found within $100 \mathrm{~nm}$ of the active zone membrane in both mossy fiber and Schaffer collateral synapses (Figure $18 \mathrm{E}$, Schaffer collateral dark grey, mossy fiber green). Mossy fiber synapses in acute slices also harbored a comparable number of docked DCVs to age-matched slice cultures (Figure $18 \mathrm{~F}$ 

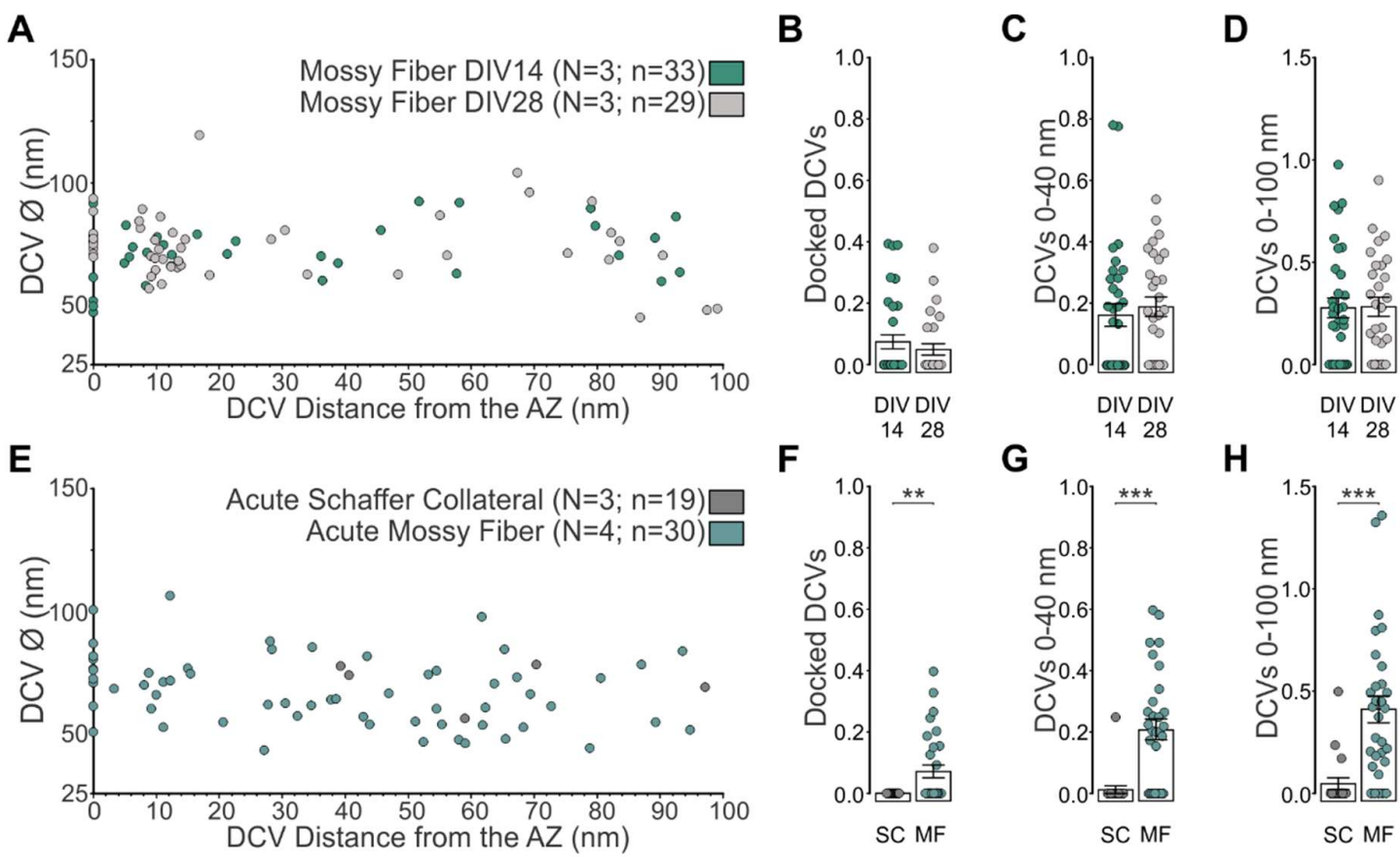

Figure 18. Spatial distribution of DCVs in mossy fiber synapses in slice cultures and acute slice preparations.

(A-D) Analysis of DCV distribution in mossy fiber synapses from hippocampal slice cultures at DIV14 (green) and DIV28 (gray). (E-H) Analysis of DCV distribution in Schaffer collateral and mossy fiber synapses from acute slice preparations from P18 wild-type mice. (A, E) DCV diameters and their respective distance to the active zone membrane. (B, F) Scatter plot of the number of docked DCVs normalized to active zone area. (C) D, G, H) Scatter plots of the number of DCVs within $40 \mathrm{~nm}(\mathbf{C}, \mathbf{G})$ and $100 \mathrm{~nm}(\mathbf{D}, \mathbf{H})$ of the active zone membrane. Statistical significance is represented as *, p<0.05; **, p<0.005; ***, p<0.001. N=number of cultures (A-D), number of animals (E-H); $n=$ number of active zones. See Tables $15-20$ for full statistical analyses.

acute slice, $0.072 \pm 0.021$, MF; compared to DIV14 slice culture, Figure $18 \mathrm{~B})$. In the acute slice preparation, DCVs were evenly distributed within $100 \mathrm{~nm}$ of the active zone membrane and did not cluster within $20 \mathrm{~nm}$ of the active zone membrane (Figure $18 \mathrm{E}$ ). The number of DCVs within $40 \mathrm{~nm}$ of the active zone membrane was comparable between mossy fiber synapses in acute slices and in age-matched slice cultures (Figure 18 G, $0.013 \pm 0.013$, SC; $0.209 \pm 0.034$, MF; $p<0.001$; compared to DIV14, Figure $18 \mathrm{C}$ ). However, the density of DCVs within $100 \mathrm{~nm}$ was higher in the acute slice preparation than in mossy fiber synapses from hippocampal slice cultures at DIV14 (Figure $18 \mathrm{H} ; 0.048 \pm 0.029, \mathrm{SC} ; 0.411 \pm 0.065$, MF; $p<0.001$ ). In 19 Schaffer collateral synapses, there were five DCVs measured within $100 \mathrm{~nm}$ of the active zone; the majority of which were located more than $40 \mathrm{~nm}$ from the active zone membrane (Figure 18 $E, G, H)$. 
My findings indicate that DCV docking at hippocampal mossy fiber synapses also occurs in vivo and therefore does not represent an artifact introduced by the slice culture procedure. Moreover, my comparative analysis indicates that DCV docking directly at the active zone membrane is specific to, or occurs considerably more frequently at, mossy fiber synapses.

\subsubsection{Munc13 priming proteins are essential for DCV docking at mossy fiber synapses and lead to accumulation of DCVs in proximity to the active zone} Genetically silenced Munc13-1/2 DKO mossy fiber synapses exhibited a complete loss of docked synaptic vesicles (Figure 11 F), giant vesicles (Figure $15 \mathrm{G}$ ), and DCVs (Figure 19 B). Moreover, DCVs in mossy fiber synapses from Munc13-1/2 DKO slice cultures accumulated between 10 and $20 \mathrm{~nm}$ from the active zone (Figure 19 A), as previously observed for synaptic vesicles (Figure $11 \mathrm{~F}$ ) and giant vesicles (Figure $15 \mathrm{E}$ ). The spatial density of docked DCVs was comparatively lower in Munc13-1/2 CTRL slices compared to wild-type slices of a similar age (Figure $19 \mathrm{~B} ; 0.077 \pm 0.035, \mathrm{CTRL} ; 0 \pm 0, \mathrm{MF} ; \mathrm{p}=0.10$ ), presumably due to the relatively small number of tomograms analyzed in this experiment. Despite the lack of docked DCVs in Munc13-1/2 DKO mossy fiber synapses, the density of DCVs within $40 \mathrm{~nm}$ of the active zone was comparable with that of Munc13-1/2 CTRL littermates (Figure 19 C; $0.191 \pm 0.054$, CTRL; $0.343 \pm 0.072$, DKO; $p=0.15$ ). Unexpectedly, Munc13-1/2 DKO mossy fiber synapses had a higher accumulation of DCVs within $100 \mathrm{~nm}$ of the active zone compared to CTRL littermates (Figure 19 D; $0.252 \pm 0.061, C T R L ; 0.643 \pm 0.095$, DKO; $p=0.002$ ). The accumulation of DCVs in Munc13-1/2 DKO mossy fiber synapses was the highest measured in mossy fiber synapses from hippocampal slice cultures.

These results indicate that Munc13 priming proteins are essential for DCV docking at hippocampal mossy fiber active zones. Moreover, the membrane-proximal accumulation of DCVs in Munc13-deficient mossy fiber synapses indicates that DCVs may undergo a tethering step prior to docking and priming at active zones. Finally, increased abundance of DCVs within $100 \mathrm{~nm}$ of the active zone membrane in Munc13-deficient mossy fiber synapses provides indirect evidence of continued anterograde transport of DCVs from the soma even in the absence of synaptic transmission. Whether increased abundance reflects the loss of basal DCV 
A

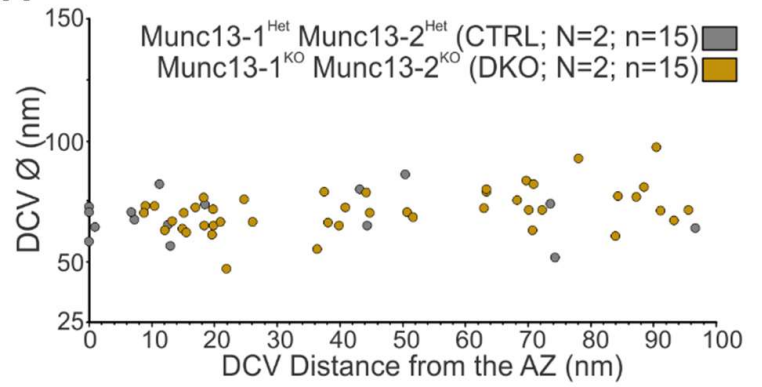

E

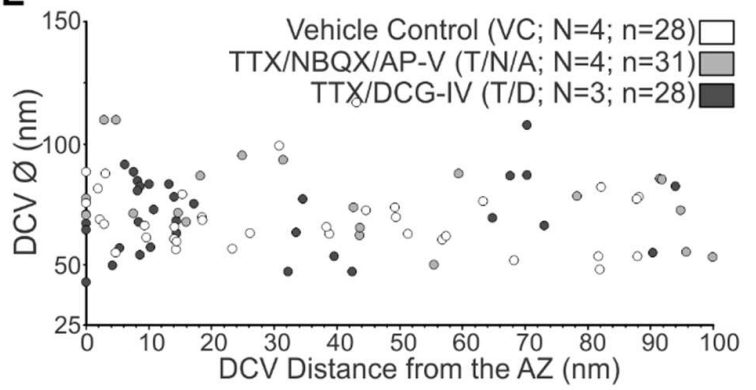

I

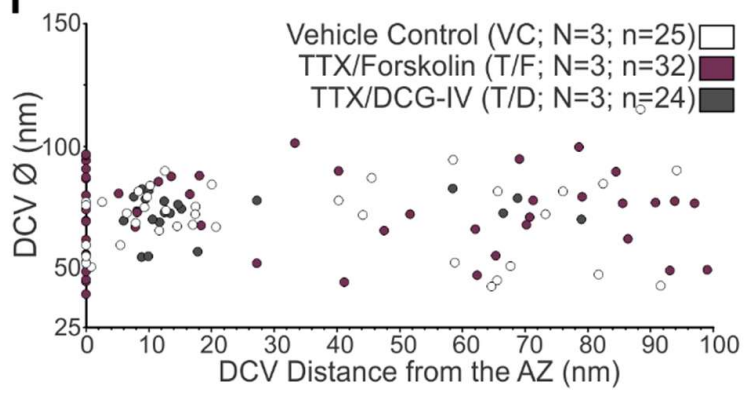

B

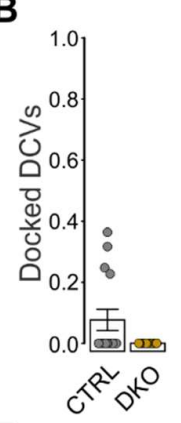

$\mathbf{F}$
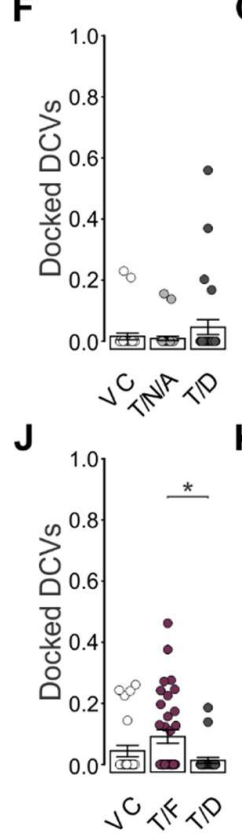

C

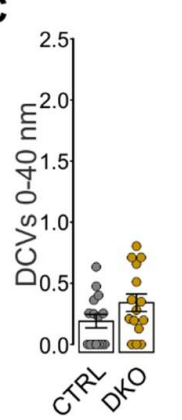

G

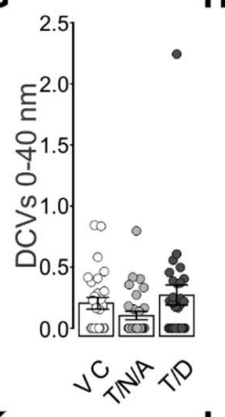

H
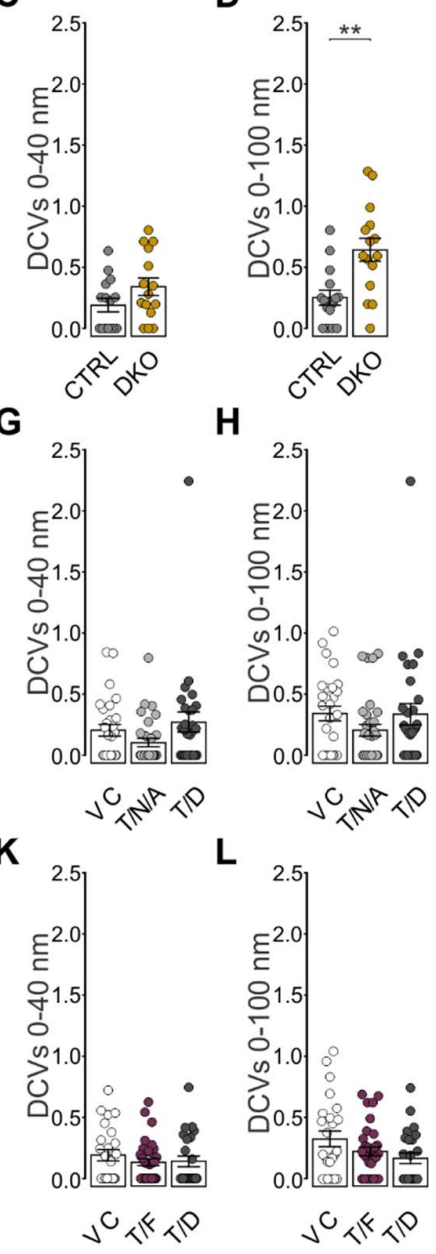

$\mathbf{L}$

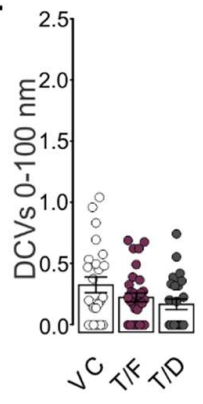

Figure 19. Spatial distribution of DCVs in mossy fiber synapses in genetically and pharmacologically manipulated slice cultures.

(A-D) Analysis of DCV distribution in mossy fiber synapses from Munc13-1/2 DKO and CTRL slice cultures at DIV28. (E-H) Analysis of DCV distribution in mossy fiber synapses after a 10 minute pharmacological silencing of slice cultures at DIV14 with one of three conditions: 1) T/N/A: TTX, to block sodium-propagated action potentials, supplemented with either NBQX and D-AP5 to block AMPA and NMDA receptor blockers, respectively; 2) T/D: TTX with DCG-IV, an mGluR2 receptor agonist that specifically blocks synaptic transmission in mossy fiber synapses (Kamiya and Ozawa, 1999); and 3) VC: comprised of slice culture medium. (I-L) Analysis of DCV distribution in mossy fiber synapses after a 15 minute treatment with either: 1) T/F: TTX, to block sodium propagated action potentials, and forskolin, an activator of AC1 which increases presynaptic cAMP concentrations (Chavez-Noriega and Stevens, 1994; Dixon and Atwood, 1989; Seamon et al., 1983) thus increasing synaptic transmission (Evans and Morgan, 2003; Seino and Shibasaki, 2005; Weisskopf et al., 1994); 2) T/D: TTX with DCG-IV, and mGluR2 receptor agonist that specifically blocks synaptic transmission in mossy fiber synapses (Kamiya and Ozawa, 1999); and 3) VC: comprised of TTX in slice culture medium in hippocampal slice cultures at DIV28. (A, E, I) Diameters of all DCVs analyzed and their respective distance to the active zone membrane. (B, F, J) Scatter plot of the number of docked DCVs normalize to active zone area. (C, D, G, H, K, L) Scatter plots of the number of DCVs within $40 \mathrm{~nm}(\mathbf{C}, \mathbf{G}, \mathbf{K})$ and $100 \mathrm{~nm}(\mathbf{D}, \mathbf{H}, \mathbf{L})$ of the active zone membrane normalized to active zone area. Statistical significance is represented as $*, p<0.05 ; * *, p<0.005 ; * * *, p<0.001$. $N=$ number of cultures; $n=$ number of active zones. See Tables 23-28 for full statistical analyses.

fusion in Munc13-1/2 DKO mossy fiber terminals, or a compensatory increase in the 
production and trafficking of DCVs to mossy fiber terminals remains to be determined.

\subsubsection{Acute pharmacological silencing of mossy fiber synapses does not change the accumulation and distribution of DCVs}

To determine whether DCV docking was influenced by spontaneous network activity, wildtype hippocampal slice cultures at DIV14 were pharmacologically silenced shortly before cryofixation. Three conditions were analyzed in this experiment: 1) T/N/A: medium supplemented with TTX, to block sodium-propagated action potentials, and NBQX and D-AP5 to block AMPA and NMDA receptor blockers, respectively; 2) T/D: medium supplemented with TTX and DCG-IV, an mGluR2 receptor agonist that specifically blocks synaptic transmission in mossy fiber synapses (Kamiya and Ozawa, 1999); and 3) VC, comprised of slice culture medium applied in the same manner as the pharmacologically treated slices prior to HPF. The spatial distribution of DCVs in mossy fiber synapses were comparable across the three treatment conditions (Figure $19 \mathrm{E}$ ). The spatial density of docked DCVs at mossy fiber active zones was unchanged between pharmacologically silenced and control slices (Figure $19 \mathrm{~F} ; 0.014 \pm 0.010, \mathrm{VC} ; 0.020 \pm 0.013, \mathrm{~T} / \mathrm{N} / \mathrm{A} ; 0.043 \pm 0.023, \mathrm{~T} / \mathrm{D} ; \mathrm{p}=0.63)$. The abundance of DCVs within $40 \mathrm{~nm}$ (Figure $19 \mathrm{G} ; 0.260 \pm 0.048$, VC; $0.104 \pm 0.034, \mathrm{~T} / \mathrm{N} / \mathrm{A} ; 0.271 \pm 0.082, \mathrm{~T} / \mathrm{D}$; $\mathrm{p}=0.05$ ) or within $100 \mathrm{~nm}$ of mossy fiber active zones (Figure $19 \mathrm{H} ; 0.342 \pm 0.060, \mathrm{VC} ; 0.205 \pm$ $0.049, \mathrm{~T} / \mathrm{N} / \mathrm{A} ; 0.337 \pm 0.087, \mathrm{~T} / \mathrm{D} ; \mathrm{p}=0.17$ ) was comparable between pharmacologically silenced and control slices.

These findings indicate that acute pharmacological blockade of spontaneous network activity does not substantially alter the spatial distribution of DCVs in mossy fiber synapses. Classically, synaptic DCV fusion occurs during states of persistent, high activity (i.e. multiple trains of high frequency stimulation; van de Bospoort et al., 2012; Farina et al., 2015) and it is

likely spontaneous network activity does not involve neuropeptide signaling in the hippocampal mossy fiber-CA3 synapse.

\subsubsection{Pharmacological manipulation of presynaptic cAMP affects DCV distribution and docking in mossy fiber synapses}

To determine whether presynaptic cAMP had an effect on DCV organization in mossy fiber synapses, I treated wild-type slice cultures at DIV28 with drug cocktails to either increase or decrease presynaptic cAMP. Three conditions were analyzed in this experiment: 1) T/F: TTX, 
to block sodium propagated action potentials, and forskolin, an activator of AC1 which increases the production of presynaptic cAMP (Chavez-Noriega and Stevens, 1994; Dixon and Atwood, 1989; Seamon et al., 1983) thus increasing synaptic transmission (Evans and Morgan, 2003; Seino and Shibasaki, 2005; Weisskopf et al., 1994); 2) T/D: TTX with DCG-IV, an mGluR2 receptor agonist that specifically blocks synaptic transmission in mossy fiber synapses (Kamiya and Ozawa, 1999); and 3) VC: comprised of TTX in slice culture medium. DCVs were observed in mossy fiber synapses after pharmacological manipulations of presynaptic cAMP, as well as in VC conditions (Figure 19 I). Forskolin-induced increases in presynaptic cAMP caused an increase in the spatial density of docked DCVs and a decrease in DCV docking upon reduction of presynaptic cAMP with DCG-IV (Figure $19 \mathrm{~J} ; 0.045 \pm 0.019, \mathrm{VC} ; 0.092 \pm 0.022, \mathrm{~T} / \mathrm{F}$; $0.014 \pm 0.009, T / D ; p=0.014)$. These findings indicate that $D C V$ docking is regulated by CAMPdependent mechanisms operating very close to the active zone, since the density of membrane-proximal DCVs within $40 \mathrm{~nm}$ of the active zone (Figure $19 \mathrm{~K} ; 0.192 \pm 0.045$, VC; $0.133 \pm 0.028, \mathrm{~T} / \mathrm{F} ; 0.141 \pm 0.043, \mathrm{~T} / \mathrm{D} ; \mathrm{p}=0.61$ ) or within $100 \mathrm{~nm}$ of the active zone (Figure $19 \mathrm{~L} ; 0.328 \pm 0.064, \mathrm{VC} ; 0.225 \pm 0.036, \mathrm{~T} / \mathrm{F} ; 0.170 \pm 0.044, \mathrm{~T} / \mathrm{D} ; \mathrm{p}=0.19$ ) was not significantly different between forskolin- or DCG-IV-treated slice cultures.

These findings indicate that acute pharmacological manipulations known to induce changes in mossy fiber synaptic transmission via manipulation of presynaptic CAMP concentrations result in corresponding changes in DCV docking at active zone membranes. It is tempting to speculate that forskolin-induced increases in DCV docking contribute to CAMP-mediated increases in release probability, since neuropeptides have been implicated in the modulation of mossy fiber transmission (Henze et al., 2000; Salin et al., 1995; Weisskopf et al., 1993). However, alternative possibilities, including the DCV-mediated delivery of active zone components during de novo active zone formation (Sorra et al., 2006; Tao et al., 2018a) must also be considered. 


\subsection{Morphological RRP estimates from past and present studies}

This is the first study to systematically investigate presynaptic mossy fiber ultrastructure and the organization of vesicles at active zone release sites using electron tomography. In this section, I extrapolated the spatial density and dimensions of docked vesicles reconstructed in tomographic subvolumes to the level of individual active zones and to the level of entire presynaptic mossy fiber terminals. Using these estimates, I compared my data with previous morphological (Rollenhagen et al., 2007) and functional (Hallermann et al., 2003; Midorikawa and Sakaba, 2017) estimates of RRP size in hippocampal mossy fiber synapses.

To compare the present study to past studies, I first normalized my data by multiplying the relative spatial density of docked synaptic vesicles, giant vesicles, and DCVs, respectively, to the average active zone area reported by Rollenhagen and colleagues (mean active zone area $=0.12 \mu \mathrm{m}^{2}$ determined by 3D serial section EM; Rollenhagen et al., 2007). In mossy fiber synapses at DIV14, I calculated approximately 5.5 synaptic vesicles, 1.4 giant vesicles, and 0.9 DCVs docked per active zone (Figure 11). At DIV28, approximately 11.6 synaptic vesicles, 1.1 giant vesicles, and 0.6 DCVs docked per active zone (Figure 12). The total number of active zones per mossy fiber bouton determined by 3D serial section EM reconstructions of entire terminals from past studies ranges from 18-45 (as many as 37 active zones reported by Chicurel and Harris, 1992; as many as 45 active zones and average of 29.75 active zones reported by Rollenhagen et al., 2007; an average of 25.3 active zones reported by Sai et al., 2017). Assuming an average of 29.75 active zones per mossy fiber bouton, approximately 163 synaptic vesicles, 42 giant vesicles, and 27 DCVs would be morphologically docked per mossy fiber bouton at DIV14. At DIV28, approximately 345 synaptic vesicles, 33 giant vesicles, and

Table 11. Estimating the total change in membrane capacitance of docked and membrane-proximal vesicles in cultured mossy fiber synapses at DIV14.

\begin{tabular}{|c|c|c|c|c|c|}
\hline $\begin{array}{l}\text { MF Synapses at } \\
\text { DIV14 }\end{array}$ & Vesicle Type & $\begin{array}{l}\text { Mean Diameter } \\
(\mathrm{nm})\end{array}$ & Mean \#/AZ & $\begin{array}{c}\text { Surface Area/AZ } \\
\left(\mathrm{nm}^{2}\right)\end{array}$ & $\begin{array}{c}\text { Estimated } \Delta \mathrm{C}_{\mathrm{m}} / \\
\text { Bouton (fF) }\end{array}$ \\
\hline \multirow{4}{*}{ Docked Vesicles } & $\mathrm{SV}(\varnothing<60 \mathrm{~nm})$ & 46.8 & 5.5 & 37785 & \\
\hline & $\mathrm{GV}(\varnothing>60 \mathrm{~nm})$ & 85.5 & 1.4 & 32558 & \\
\hline & DCV & 68.4 & 0.9 & 13244 & \\
\hline & Total & & & 83587 & $24-37$ \\
\hline \multirow{4}{*}{$\begin{array}{l}\text { Vesicles } \\
0-40 \mathrm{~nm}\end{array}$} & SV $(\varnothing<60 \mathrm{~nm})$ & & 20.6 & 132322 & \\
\hline & $\mathrm{GV}(\varnothing>60 \mathrm{~nm})$ & & 4.3 & 98522 & \\
\hline & DCV & & 2.0 & 34014 & \\
\hline & Total & & & 264858 & $78-119$ \\
\hline
\end{tabular}


18 DCVs would be docked per mossy fiber bouton. Based on the mean diameter of respective vesicle types obtained from tomographic reconstructions, I calculated the respective vesicular surface areas that would be integrated with the plasma membrane upon fusion of all docked vesicles (Table 11, DIV14; Table 12, DIV28). Based on a specific membrane capacitance of $1 \mu \mathrm{F} / \mathrm{cm}^{2}$ (Gentet et al., 2000; Hallermann et al., 2003; Midorikawa and Sakaba, 2017), my data would predict membrane capacitance increases of between 24-37 fF at DIV14 (Table 11) and 33-50 fF at DIV28 (Table 12) if all morphologically docked vesicles were to fuse during a step depolarization. These estimates appear compatible with the membrane capacitance increase reported to correspond with depletion of the RRP in cultured mossy fiber terminals (Table 13; Midorikawa and Sakaba, 2017).

Table 12. Estimating the total change in membrane capacitance of docked and membrane-proximal vesicles in cultured mossy fiber synapses at DIV28.

\begin{tabular}{|c|c|c|c|c|c|}
\hline $\begin{array}{l}\text { MF Synapses at } \\
\text { DIV28 }\end{array}$ & Vesicle Type & $\begin{array}{l}\text { Mean Diameter } \\
(\mathrm{nm})\end{array}$ & Mean \#/AZ & $\begin{array}{c}\text { Surface Area/AZ } \\
\left(\mathrm{nm}^{2}\right)\end{array}$ & $\begin{array}{c}\text { Estimated } \\
\text { Change in } \\
\text { Capacitance/ } \\
\text { Bouton (fF) }\end{array}$ \\
\hline \multirow{4}{*}{ Docked Vesicles } & SV $(\varnothing<60 \mathrm{~nm})$ & 45.7 & 11.6 & 76136 & \\
\hline & $\mathrm{GV}(\varnothing>60 \mathrm{~nm})$ & 84.3 & 1.1 & 24898 & \\
\hline & DCV & 78.0 & 0.6 & 11471 & \\
\hline & Total & & & 112505 & $33-50$ \\
\hline \multirow{4}{*}{ Vesicles $0-40 \mathrm{~nm}$} & SV $(\varnothing<60 \mathrm{~nm})$ & & 25.1 & 161281 & \\
\hline & $\mathrm{GV}(\varnothing>60 \mathrm{~nm})$ & & 3.0 & 68827 & \\
\hline & DCV & & 2.3 & 39649 & \\
\hline & Total & & & 269757 & $80-121$ \\
\hline
\end{tabular}

Based on the assumption that membrane-proximal vesicles positioned upstream of docking and priming might be recruited to the functional RRP and elicited to fuse during strong depolarizing pulses, I calculated the theoretical change in membrane capacitance if all vesicles within $0-40 \mathrm{~nm}$ of the active zone membrane were to fuse with the presynaptic membrane at DIV14 (Table 11) and DIV28 (Table 12). The theoretical change in membrane capacitance at DIV28 would range between 80 and $121 \mathrm{fF}$, very close to the larger change in presynaptic

Table 13. Published presynaptic capacitance studies of mossy fiber boutons.

\begin{tabular}{|c|c|c|c|c|}
\hline Method & Publication & Preparation & $\begin{array}{c}\text { Pulse } \\
\text { Duration }\end{array}$ & $\Delta \mathrm{C}_{\mathrm{m}} /$ Bouton (fF) \\
\hline \multirow{3}{*}{ Electrophysiology } & (Hallermann et al., 2003) & Rat, acute slice & $30 \mathrm{~ms}$ & 100 \\
\hline & (Midorikawa and Sakaba, 2017) & Rat, acute slice & $30 \mathrm{~ms}$ & 50 \\
\hline & (Midorikawa and Sakaba, 2017) & Rat, dissociated & $30 \mathrm{~ms}$ & 30 \\
\hline
\end{tabular}


membrane capacitance recorded by Hallermann and colleagues (Table 13; Hallermann et al., 2003).

These findings indicate that the number of morphologically docked vesicles correlates relatively well with functional estimates of the RRP assessed by presynaptic capacitance recordings in hippocampal mossy fibers. However, it indicates that strong stimuli applied by direct depolarization of the presynaptic terminal likely trigger fusion of more than just the membrane-attached pool of docked vesicles. It should however be noted that hippocampal mossy fibers exhibit a very low initial release probability (Figure 8) (Lawrence et al., 2004; Nicoll and Schmitz, 2005; Salin et al., 1996; Vyleta and Jonas, 2014), and that action potentials likely evoke fusion from only a subpopulation of the docked vesicle pool. It will therefore be interesting to investigate potential heterogeneity in the vesicular release probability of different vesicle classes in future studies. Nevertheless, my work represents the first study to take the morphological heterogeneity of presynaptic vesicle pools into account in a systematic ultrastructural analysis of mossy fiber active zone organization. 


\section{Discussion}

\subsection{Overview}

Synaptic transmission operates by a stereotypical principle involving multiple molecularly regulated steps prior to vesicle fusion and transmitter release at presynaptic active zones. This complexity provides multiple opportunities for the modulation of synaptic transmitter release efficacy. Several mechanisms have been postulated to play a role in determining synaptic release probability, including the physical distance between calcium influx and calcium sensor (Chen et al., 2015), the type of calcium buffer present (Müller et al., 2005; Vyleta and Jonas, 2014), proximity of mitochondria to active zone release sites (Brodin et al., 1999; Smith et al., 2016), the intrinsic properties of synaptic vesicles related to the state of the release machinery (Cano et al., 2012), and the availability of functionally primed and release-competent vesicles (Imig et al., 2014). Based on several converging lines of experimentation that indicate considerable overlap between pools of morphologically docked and functionally primed vesicles (Imig et al., 2014; Rosenmund and Stevens, 1996; Schikorski and Stevens, 1997, 2001; Siksou et al., 2009a), I performed experiments to address the question of whether the availability of morphologically docked synaptic vesicles contributes to differences in the initial synaptic release probability of hippocampal Schaffer collateral and mossy fiber synapses. I adopted a methodological approach combining hippocampal organotypic slice culture, HPF, AFS, and electron tomography to accurately resolve synaptic ultrastructure in a near-native and 3D context. In my comparative ultrastructural analysis of presynaptic vesicle organization at wild-type hippocampal Schaffer collateral and mossy fiber

synapses at rest, I discovered that low release probability mossy fiber synapses are characterized by a low spatial density of docked vesicles at individual active zones. Mossy fiber synapses were additionally distinguished by the presence of a prominent membraneproximal and potentially tethered pool of vesicles. I hypothesize that this membrane-proximal pool is ideally situated to rapidly resupply the pool of docked and primed vesicles during sustained synaptic activity and that it likely contributes to the characteristic facilitation exhibited by mossy fiber synapses (Marchal and Mulle, 2004; Nicoll and Schmitz, 2005; Salin et al., 1996).

My systematic ultrastructural analysis revealed that three distinct types of vesicle dock at mossy fiber active zones, including synaptic vesicles, giant vesicles, and DCVs. Moreover, I 
demonstrate that this morphological heterogeneity within the docked vesicle pool is also present at mossy fiber active zones in vivo and therefore not an artifact of the slice culture preparation. Although the origin and functional relevance of giant vesicles remains to be determined, my data support the hypothesis that giant vesicles contain neurotransmitter and contribute to glutamatergic signaling at mossy fiber active zones. By extending my analyses to include genetically silenced synapses, I demonstrated that similar Munc13-dependent molecular mechanisms operate to dock and functionally prime all three species of vesicle at mossy fiber synapses.

Consistent with a significant overlap between pools of morphologically docked and functionally primed vesicles (Imig et al., 2014; Rizzoli and Betz, 2004; Rosenmund and Stevens, 1996; Schikorski and Stevens, 1997, 2001; Siksou et al., 2009a), my data indicate a relatively close correlation between numbers of membrane attached vesicles and functional RRP estimates estimated from presynaptic capacitance recordings (Hallermann et al., 2003; Midorikawa and Sakaba, 2017). Importantly, my data indicate that protocols applying strong stimulation, such as direct depolarization of the presynaptic terminal, likely trigger the fusion of both membrane-proximal and docked vesicles. Since postsynaptic responses elicited by presynaptic action potentials appear to result from the fusion of only a small subpopulation of the total docked pool (Bekkers and Stevens, 1995; Gustafsson et al., 2019; Hanse and Gustafsson, 2001; Stevens and Tsujimoto, 1995; Vyleta and Jonas, 2014), my data indicate heterogeneity in the vesicular release probability of membrane-attached vesicles docked at mossy fiber active zones.

In addition to their morphological characteristics, hippocampal mossy fiber synapses are functionally distinguished by their almost exclusive reliance on presynaptically expressed plasticity mechanisms (Lawrence et al., 2004; Maccaferri et al., 1998; Nicoll and Schmitz, 2005; Salin et al., 1996; Toth et al., 2000). I investigated whether pharmacological manipulation of presynaptic cAMP (Huang et al., 1994b; Seino and Shibasaki, 2005; Tzounopoulos et al., 1998; Villacres et al., 1998; Weisskopf et al., 1994) caused corresponding changes in presynaptic vesicle organization at mossy fiber active zones. My analysis of forskolin-treated slices revealed only subtle changes in synaptic vesicle organization, supporting the supposition that CAMP-mediated increases in mossy fiber synaptic transmission operate via molecular regulation of post-docking processes. However, I 
observed a two-fold forskolin-induced increase in DCV docking at mossy fiber active zones that implicates neuropeptide signaling as a potential contributing factor involved in presynaptic mechanisms of mossy fiber plasticity. 


\subsection{Methodological considerations}

The main objective of this study was to examine whether the number of morphologically docked synaptic vesicles corresponds to differences in release probability exhibited between Schaffer collateral (weakly facilitating or depressing; phasic) and mossy fiber (strongly facilitating, tonic) synapses in the hippocampus. I asked the question: Do mossy fiber and Schaffer collateral synapses differ not only in the total number of active zones and synaptic vesicles, but also in the organization of vesicles at individual active zones? My results reveal previously unreported ultrastructural characteristics of hippocampal mossy fibers and demonstrate a clear correlation between the availability of docked and primed vesicles and the initial release probabilities of hippocampal mossy fiber and Schaffer collateral synapses. Although this fundamental ultrastructure-function relationship has been examined in a variety of model systems and synapse types, discrepancies between studies have prevented a general consensus (Atwood and Karunanithi, 2002; Eltes et al., 2017; Govind et al., 1994; Holderith et al., 2012; Millar et al., 2002; Schikorski and Stevens, 1997; Xu-Friedman et al., 2001). Nevertheless, I was motivated to revisit this question, particularly since mossy fiber synapses have not previously been scrutinized at the level of resolution permitting accurate discrimination of functionally distinct membrane-proximal vesicle pools. Several important methodological considerations need to be taken into account in order to interpret and compare my data with the results of previous studies obtained using alternative experimental approaches to address synaptic ultrastructure-function relationships. In the following section, I classify and discuss these limitations in the context of sample preparation for electron microscopic analysis, the mode of image acquisition, and of analysis of synaptic ultrastructure.

\subsubsection{Sample preparation}

In contrast to many previous studies based on the analysis of aldehyde-fixed tissue, my thesis work was almost entirely founded upon the ultrastructural analysis of tissue that had been rapidly cryo-fixed by HPF in a living, unfixed state. To assess the potential benefits of this approach in the context of synaptic vesicle docking analyses, I compared the effects of aldehyde fixation on synaptic vesicle docking in hippocampal mossy fiber synapses prepared for ultrastructural analysis by i) transcardial perfusion of aldehyde fixative cocktails, ii) 
immersion of organotypic slice cultures in aldehyde fixative cocktails, and by iii) HPF of organotypic slice cultures.

I found that in comparison to high-pressure frozen organotypic slices, the abundance of membrane proximal and docked vesicles was severely reduced in perfusion fixed tissue. Moreover, I observed that the extent of perturbation correlated with the fixative osmolarity, perhaps reflecting the sensitivity of the synaptic RRP, and presumably of the vesicle fusion apparatus, to osmotic pressure (Bekkers and Stevens, 1995; Rosenmund and Stevens, 1996; Stevens and Tsujimoto, 1995). The osmolarity of aldehyde fixative cocktails typically exceeds that of physiological buffers (Hayat, 1981). In my study, two different fixative cocktails were used: PF1 [4\% PFA, 2.5\% GA in $0.1 \mathrm{M} \mathrm{PB}, \mathrm{pH} 7.4,4^{\circ} \mathrm{C}$; approximately 1900 mOsm (Hayat,

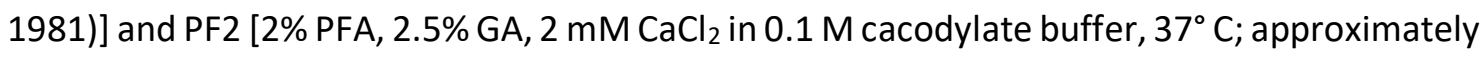
1200 mOsm (Hayat, 1981)]. Another consideration concerns the speed with which the synaptic ultrastructure is immobilized. Aldehyde fixation via transcardial perfusion occurs relatively slowly because the fixative must diffuse through tissue while cross-linking proteins in an outside-in direction (Hopwood, 1969).

My observation that membrane proximal vesicles are depleted in perfusion-fixed mossy fiber synapses is in line with a previous study comparing the ultrastructural organization of synapses in the somatosensory cortex prepared by transcardial aldehyde perfusion fixation and by HPF of acutely dissected tissue (Korogod et al., 2015). Korogod and colleagues used serial section transmission EM, electron tomography, and focused ion-beam scanning electron microscopy (FIB-SEM) to compare the effects of perfusion fixation of aldehyde cocktails to high-pressure frozen acute slice preparations (Korogod et al., 2015). Using electron tomography, they found a reduction in membrane-proximal synaptic vesicles in reconstructed tomograms of synaptic profiles from perfusion-fixed compared to cryo-fixed tissue (Korogod et al., 2015). I interpret the loss of membrane proximal vesicles in my study as a consequence of aldehyde-triggered vesicle fusion rather than a rapid redistribution from the active zone membrane. This hypothesis is supported by my frequent observation of omega-shaped membrane profiles at active zones in the perfusion-fixed mossy fiber synapses, which is indicative of full-collapse synaptic vesicle fusion. The notion that aldehyde exposure can trigger vesicle fusion was initially proposed by Smith and Reese (Smith and Reese, 1980), who observed that perfusion of different aldehydes: GA, formaldehyde, or 
crotonaldehyde, caused an increase in post-synaptic endplate potentials recorded from the frog neuromuscular junction, as well as an increase in pits in proximity to the active zone attributed to fusing vesicles (Smith and Reese, 1980). A contrasting view was presented by Rosenmund and Stevens, who recorded EPSCs in dissociated hippocampal neurons and found minimal synaptic vesicle fusion upon fast perfusion of $2 \%$ GA (Rosenmund and Stevens, 1997). They found that fixation was rapid and caused a $10 \%$ depletion of the functional RRP (Rosenmund and Stevens, 1997), indicating that the speed at which synaptic ultrastructure is immobilized is likely an important factor involved in the effect of aldehydes on docked vesicle pools.

Consistent with this view, the number of docked vesicles at synapses in immersion-fixed and high-pressure frozen slices was comparable at the surface of the tissue (5-11 $\mu \mathrm{m}$ from the tissue surface; Table 30, Figure $21 \mathrm{~A}-\mathrm{E}$ ), whereas the depletion of docked vesicle pools became apparent in synapses located deeper in the tissue (20-22 $\mu \mathrm{m}$ from tissue surface; Table 31; Figure $21 \mathrm{~F}-\mathrm{I}$ ). Since identical aldehyde-based fixative cocktails were used for perfusion and immersion fixation experiments, these data imply that synaptic ultrastructure in surfaceexposed synapses is immobilized before the osmotic pressure exerted by the fixatives can manifest and trigger vesicle fusion. However, additional analysis is required to assess whether docked vesicle pools are maintained only at the expense of membrane-proximal, putatively tethered vesicles in surface-exposed and immersion fixed synapses. Regardless, my observations, which appear consistent with the aforementioned study by Rosenmund and Stevens (Rosenmund and Stevens, 1997), identify synapse location and accessibility as important variables affecting the impact of immersion fixation on synaptic ultrastructure.

Taken together, the results of my study emphasize that caution must be exercised when interpreting synaptic ultrastructure in aldehyde-fixed samples and that rapid cryo-fixation is more reliable for the analysis of synaptic vesicle organization at presynaptic active zones. I cannot, however, exclude the possibility that more refined fixative compositions or perfusion protocols may limit the artifactual effects of aldehydes on vesicle organization at individual active zones. Aldehyde fixation will remain an extremely useful means of preserving synaptic ultrastructure for light and electron microscopic analysis, since many brain structures are incompatible with rapid dissection for cryo-fixation (i.e. HPF), and the dissection process itself risks mechanical trauma and anoxia (Korogod et al., 2015; Sosinsky et al., 2008). Protocols 
involving sequential aldehyde perfusion, dissection, and HPF have therefore been proposed (Sosinsky et al., 2008), the effects of aldehydes on membrane-proximal vesicle pools notwithstanding.

Future work based on my study would be to investigate the spatial distribution of synaptic vesicles within $100 \mathrm{~nm}$ of the active zone in mossy fiber synapses from immersion-fixed hippocampal slices to test whether the membrane-proximal, or tethered synaptic vesicle pools change in mossy fiber synapses after immersion fixation. It is possible that in the superficial mossy fiber synapses, the RRP was depleted by $10 \%$, as was observed in the Rosenmund and Stevens study (Rosenmund and Stevens, 1997), however, the loosely docked (LS) or tethered synaptic vesicles observed in mossy fiber synapses at rest may rapidly dock and prime during immersion fixation. To better study the effects of aldehydes on membraneproximal pools of synaptic vesicles, a full 3D study of synaptic vesicles within $100 \mathrm{~nm}$ is necessary. Furthermore, a more thorough study of the diffusion distance of aldehydes is necessary to test whether the distance of fixatives is proportional to the deficits of membrane-proximal synaptic vesicles observed in this study. This could be accomplished by comparing the change in the spatial distribution of synaptic vesicles at mossy fiber synapses closer to blood vessels with those farther away in perfusion-fixed mice.

\subsubsection{Limitations of 2D electron microscopy and advantages of 3D electron tomography}

Although conceptually simple, the accurate assessment of synaptic vesicle docking is nontrivial. Multiple factors contribute to this: (i) Synaptic vesicles are small and the defining structural feature of a docked synaptic vesicle, i.e. the point of contact between the outer lipid bilayer of a synaptic vesicle and the inner lipid bilayer of the presynaptic membrane, is considerably smaller. (ii) Synaptic membranes are inherently curved. (iii) Electron microscopic images, even from ultrathin sections (20-100 $\mathrm{nm}$ thick), represent 2D projections through a volume. (iv) Due to mechanical limitations, plastic sections are difficult to cut thinner than the approximate diameter of a synaptic vesicle $(\sim 40 \mathrm{~nm})$. In addition to the sample preparation considerations outlined in the previous section, the accuracy with which vesicle docking is assessed in electron micrographs is dependent on z-resolution. Since z-resolution is defined by plastic section thickness in 2D imaging approaches, including serial section-based 3D EM techniques, certain ambiguities are introduced and it is not possible to determine whether 
the midline of a synaptic vesicle is contained within the imaged volume. This limitation also excludes the possibility of accurate vesicle volume measurements. Nevertheless, 3D serial section EM analyses offer a larger field-of-view than electron tomography, are compatible for the reconstruction of large tissue volumes, and provide access to important quantitative information, including volume measurements of pre- and postsynaptic compartments and total numbers of active zone release sites (Chicurel and Harris, 1992; Harris and Sultan, 1995; Rollenhagen et al., 2007; Sätzler et al., 2002; Spacek and Harris, 1998; Xu-Friedman et al., 2001).

Electron tomography circumvents this limitation by achieving a higher z-resolution, which is inversely related to the number of images in the tilt-series, the tilt increment separating them, and the pixel spacing used for image acquisition (Koster et al., 1997). The final voxel dimensions achieved in tomographic slices are therefore dependent on the magnification used to acquire the tilt-series and the extent of binning used for weighted back-projection to convert the tilt series into a volume (Koster et al., 1997). Since functionally critical, but morphologically subtle, changes in vesicle organization can manifest within the range of several nanometers (Imig et al., 2014; Siksou et al., 2009a), it is important to resolve the spatial organization of synaptic vesicles as accurately as possible. For example, electron tomographic reconstructions generated in my comparative analyses revealed that mossy fiber synapses not only harbor a lower spatial density of docked vesicles at individual active zones compared to Schaffer collateral synapses, but that they are distinguished by the presence of a prominent membrane-proximal, possibly tethered, pool of synaptic vesicles. The novelty of these observations implies that the combination of good ultrastructural preservation and high resolution imaging is required to dissect functionally relevant structural features of vesicle organization at active zone release sites. Consistent with this view, previously reported differences in the numbers of docked vesicles at facilitating and depressing synapses were generated using electron tomography (Eltes et al., 2017).

In conclusion, I used electron tomography to reconstruct synaptic subvolumes in 3D with an isotropic voxel dimension of about $1.6 \mathrm{~nm}$. In comparison to conventional 2D transmission EM analysis, electron tomography provides a better z-resolution and reveals fine structural changes in the spatial distribution of synaptic vesicles at active zone release sites that have otherwise been elusive. 


\subsubsection{Vesicle docking criteria}

A potential source of discrepancy between studies examining the functional relevance of synaptic vesicle organization is the use of different criteria to classify vesicle docking in an electron micrograph. Relevant questions in such analyses become: (i) How accurately can the 'true' position of a synaptic vesicle be resolved? (ii) How close to the membrane does a vesicle need to be in order to be considered docked? (iii) How do the applied sample preparation techniques (i.e. fixation, dehydration) influence vesicle organization? Indeed, the definition of a morphologically docked synaptic vesicle is often adapted to take the limitations of the applied methodology into account.

For example, in a 3D serial section EM analysis of vesicle docking in perfusion fixed mossy fiber synapses, in order to compensate for the associated limitations in z-resolution in 50$60 \mathrm{~nm}$-thick plastic sections, Rollenhagen and colleagues measured distances between the center of vesicles to the presynaptic membrane and the mean synaptic vesicle radius measured was subtracted to calculate the closest approach (Rollenhagen et al., 2007). Since the total number of synaptic vesicles within $60 \mathrm{~nm}$ of the active zone membrane $(\sim 1200$ vesicles) were found to correlate to the number of vesicles predicted to fuse upon depletion of the RRP as assessed by presynaptic capacitance recordings ( 1400 vesicles; Hallermann et al., 2003), these vesicles were classified as belonging to the putative RRP (Rollenhagen et al., 2007). However, this putative RRP would include all vesicles within $40 \mathrm{~nm}$ of the active zone membrane resulting in an overestimation of docked synaptic vesicles.

More stringent docking criteria have been used in other 3D serial section EM studies. In an analysis of synaptic vesicle docking in the stratum oriens of the CA3 from juvenile rat hippocampi, Holderith and colleagues reconstructed entire active zones from serial $20 \mathrm{~nm}$ thick sections (Holderith et al., 2012). For a synaptic vesicle to be considered docked in this study, the distance between the middle of the lipid bilayers on the presynaptic membrane and synaptic vesicle was required to be less than $5 \mathrm{~nm}$ (Holderith et al., 2012). Since my electron tomographic analyses reveal lipid bilayers to be approximately $4 \mathrm{~nm}$ thick from center-to-center of inner and outer leaflets, the criteria used by Holderith and colleagues, 
coupled with the very thin sections imaged, provides a stringent analysis of the number of docked synaptic vesicles (Holderith et al., 2012).

In the present study and in previous work from our group using HPF, AFS, and electron tomography, docked vesicles were defined as those with no measurable distance between the synaptic vesicle membrane and the active zone membrane (Imig et al., 2014). In both studies, tomograms were acquired at 30,000x magnification and a $3 \mathrm{x}$ binning factor was used when reconstructing synaptic subvolumes, generating tomographic slices with isotropic voxel dimensions of $x, y$, and $z=\sim 1.6 \mathrm{~nm}$ (Imig et al., 2014). Morphologically docked vesicles were reported as vesicles within $0-2 \mathrm{~nm}$ of the active zone membrane. This study of Schaffer collateral synapses indicated that docked vesicles classified according to this criterion represent morphological correlates of functionally primed and fusion-competent vesicles (Imig et al., 2014). Docked vesicle pools within 0-2 nm of the membrane are massively reduced in priming and SNARE protein deficient genetic mutants (Imig et al., 2014) and the extent of reduction is highly comparable to published reductions in sucrose-evoked measurements of RRP size in respective mutants (Arancillo et al., 2013; Augustin et al., 1999; Bronk et al., 2007; Schoch et al., 2001; Varoqueaux et al., 2002; Washbourne et al., 2002). Although our workflow combining HPF and AFS circumvents many potential limitations of aldehyde fixation and room-temperature dehydration, the tissue is nevertheless dehydrated, albeit at sub-zero temperatures, and heavy metals are deposited on the lipid bilayers to enhance membrane contrast. Consequently, the possibility that these procedures could induce subtle changes in the distribution of vesicles at active zones, or even occlude the detection of very small gaps between vesicles and the presynaptic membrane, should be taken into consideration.

An alternative perspective of how synaptic vesicles interact with the presynaptic membrane has evolved with the technological development of cryo-EM, which allows the ultrastructure of synapses to be viewed in a frozen-hydrated state (Fernández-Busnadiego et al., 2010, 2013; Lučić et al., 2005; Schrod et al., 2018; Tao et al., 2018b; Zuber and Lučić, 2019). Studies employing cryo-electron tomography to resolve the active zone organization of plunge-frozen synaptosome preparations demonstrated that direct synaptic vesicle contact with the plasma membrane was only rarely observed (Fernández-Busnadiego et al., 2010). Rather, active zone proximal vesicles were connected to the membrane by multiple short filaments $(<5 \mathrm{~nm})$ 
(Fernández-Busnadiego et al., 2010). The authors interpreted vesicles in this state as potentially primed and analogous to the docked vesicles in direct contact observed in heavy metal contrasted and dehydrated preparations (Fernández-Busnadiego et al., 2010). Although aspects of the synaptosome preparation protocol likely induce some degree of structural reorganization in presynaptic vesicle pools, more recent studies corroborate several observations made in synaptosomes using cultured neurons (Schrod et al., 2018; Tao et al., 2018a, 2018b). In these experiments, dissociated neuron cultures were maintained on EM grids and vitrified by plunge freezing prior to cryo-electron tomographic analysis (Schrod et al., 2018; Tao et al., 2018b). These studies of intact synaptic boutons confirmed that direct vesicle-membrane contact was rare, and that vesicles closest to the active zone membrane were situated at a small distance spanned by multiple, short filamentous tethers (Schrod et al., 2018; Tao et al., 2018b). The molecular identity of the short filaments remains unknown and future experiments quantifying their abundance in appropriate genetic null mutants (i.e. synapses lacking SNARE components or priming proteins), will be informative.

In summary, it is important to understand the advantages and limitations of the employed methodologies when assessing synaptic vesicle docking. In my study, I used 3D electron tomography which resulted in isotropic voxel dimensions compatible to the accurate assessment of synaptic vesicle docking. I set a stringent docking criterion-no measurable distance between the membranes of synaptic vesicles and the presynaptic active zone. While electron tomography of plastic-embedded samples provides better 3D resolution than 2D transmission EM, cryo-electron tomography images biological samples with no additives such as those used for plastic embedding. An additional comparison between the spatial organization of synaptic vesicles in synapses imaged with cryo-electron tomography and electron tomography of plastic-embedded synapses would be highly informative. 


\subsection{The RRP and morphologically docked vesicles}

The question of whether morphologically docked synaptic vesicles at a given synapse can predict differences in synaptic release probability has been contentious (Atwood and Jahromi, 1978; Eltes et al., 2017; Holderith et al., 2012; Neher and Brose, 2018; Xu-Friedman and Regehr, 2003; Xu-Friedman et al., 2001). At the core of this exploration comes another question: do docked synaptic vesicles comprise the RRP of primed, fusion-competent synaptic vesicles? The fact that loss of Munc13-1 and -2 abolishes both docking and priming indicates that the number of docked vesicles (Figure 11, Figure 15, and Figure 19) serves as a reliable proxy for the number of molecularly primed vesicles (Imig et al., 2014)

It is also clear that in response to a given action potential, only a fraction of docked and therefore primed synaptic vesicles will fuse. The RRP is a vague concept that usually requires some degree of activity to extrapolate the approximate number of docked and primed synaptic vesicles. With HPF, AFS, and electron tomography of mossy fiber synapses, I defined the RRP of morphologically docked synaptic vesicles from these synapses at rest. I compared my findings to past functional studies that used presynaptic capacitance recordings of mossy fiber synapses to estimate the RRP of mossy fiber boutons (Hallermann et al., 2003; Midorikawa and Sakaba, 2017). Our theoretical RRP estimates fell within the reported range of 400-1400 synaptic vesicles (Hallermann et al., 2003; Midorikawa and Sakaba, 2017). I calculated, based on the average docked vesicle density, that approximately 320 synaptic vesicles, 37 giant vesicles and 15 DCVs are docked and primed in a given mossy fiber bouton at rest (Table 12). The authors of the previous work describing RRP estimates from presynaptic capacitance recordings of mossy fiber boutons acknowledge that they do not account for DCV fusion nor exocytosis from filopodia that extend from mossy fiber boutons and form synapses on inhibitory interneurons (Acsády et al., 1998; Hallermann et al., 2003; Midorikawa and Sakaba, 2017). The neglect of giant vesicles' potential contributions to induced capacitance changes at hippocampal mossy fiber synapses may lead to an overestimation of the RRP (Midorikawa and Sakaba, 2017; Rollenhagen et al., 2007).

I found that the total docked vesicle pool in mossy fiber synapses is several hundred vesicles. However, the number of vesicles that seem to fuse in response to an AP appears to be much smaller (Jonas et al., 1993; Lawrence et al., 2004). This implies a heterogeneous release probability among the docked and primed synaptic vesicle pool. Both fast and slow 
components of synaptic transmission have been described and the contribution to each component is provided by vesicles of certain release probabilities. This was observed in calyx of Held synapses and is partially due to the synaptic vesicle-VGCC coupling distance (Chen et al., 2015). Vesicles within the fast pool are coupled to VGCCs at a distance of $\sim 16 \mathrm{~nm}$ whereas vesicles in the slow pool were estimated to reside 30 to $100 \mathrm{~nm}$ from the calcium channels (Chen et al., 2015; Neher, 2015). A recent study looked at the freeze-fracture replica immunolabelling of VGCC distribution in two synapses in the cortex with high and low release probability (Rebola et al., 2019). They found the opposite correlation of VGCC density to release probability in that synapses with lower release probability had a higher density of VGCCs and synapses with a higher release probability had a lower abundance of VGCCs (Rebola et al., 2019). Rebola and colleagues also found that synapses with a lower release probability had a larger calcium channel-synaptic vesicle coupling distance than synapses with a higher release probability (Rebola et al., 2019). Mossy fiber synapses have a loose calcium channel-synaptic vesicle coupling distance of approximately $70 \mathrm{~nm}$, and although the total number of calcium channels has been estimated to be about 23 per active zone (Vyleta and Jonas, 2014), the actual distribution of calcium channels remains to be determined.

Another important consideration associated with presynaptic capacitance concerns control of presynaptic intracellular calcium concentrations and the distance calcium ions travel within the bouton during a given step-depolarization protocol. Indeed, the addition of calcium buffers to intracellular recording solutions, which compete with endogenous buffers within the presynaptic terminal, can profoundly influence RRP estimates. For example, in work from Hallermann and colleagues, the presynaptic calcium buffer concentration was half of that used in the work published by Midorikawa and Sakaba (0.26 mM EGTA, Hallermann et al., 2003; 0.5 mM EGTA, Midorikawa and Sakaba, 2017). The RRP estimate extrapolated from the study by Hallermann and colleagues likely contained docked and primed vesicles as well as membrane-proximal vesicles (loosely-docked) that rapidly primed upon sustained calcium influx, therefore inflating the RRP estimate (Hallermann et al., 2003). For this reason, I incorporated loosely-docked synaptic vesicles, synaptic vesicles within $0-40 \mathrm{~nm}$ of the active zone, into my RRP estimate (Table 12). The theoretical change in membrane capacitance when including loosely-docked synaptic vesicles is within the range of the upper estimates from presynaptic capacitance measurements (Table 12; Table 13; Hallermann et al., 2003). 
My study supports the finding that morphologically docked synaptic serve as a reliable proxy for the RRP of a given synapse (Imig et al., 2014; Siksou et al., 2009a). However, in the tomograms of Schaffer collateral and mossy fiber synapses reconstructed and analyzed in this study, each tomogram contained a fraction of an entire active zone. Therefore, to make RRP estimates per active zone and per bouton, I relied on active zone areas reported from past morphological studies (Chicurel and Harris, 1992; Rollenhagen et al., 2007). Ideally, electron tomograms of serial semi-thin ( $200 \mathrm{~nm}$-thick) sections could be used to reconstruct entire active zones at both Schaffer collateral and mossy fiber synapses to provide a more accurate estimate of the RRP.

\subsubsection{Limitations of RRP estimates}

The RRP has been historically assessed by functional means and corresponds to the number of vesicles that fuse with the synapse in response to strong, vesicle-depleting stimuli (Kaeser and Regehr, 2017; Neher, 2015). Several approaches assess the RRP of a particular neuron or synapse: high-frequency stimulation (Schneggenburger et al., 1999), presynaptic membrane capacitance recordings (Neher and Marty, 1982), optical approaches including FM1-43 dye uptake (Rizzoli and Betz, 2004; Schikorski and Stevens, 2001) and phlourins (Ariel and Ryan, 2010), and perfusion of hypertonic sucrose solutions (Rosenmund and Stevens, 1996).

Most commonly, the RRP for synapses in acute slice preparation is measured by recording action potential-evoked EPSCs during high-frequency stimulation paradigms to compare the responses to the quantal content of a single synaptic vesicle from spontaneous fusion events and back extrapolate the number of vesicles that form the RRP (Schneggenburger et al., 1999; Thanawala and Regehr, 2013). However, it is an indirect measurement of vesicle fusion as it depends on the detection of neurotransmitter molecules by postsynaptic receptors. Moreover, during long high-frequency stimulation trains the RRP is constantly refilled (Neher, 2015).

Optical approaches offer a sensitive way to study the RRP in neurons and to determine which vesicles participate in the vesicle cycle. For example, a key study from Rizzoli and Betz used photoconversion of FM1-43 dye uptake via endocytosis after pool-depleting simulation of the frog neuromuscular junction to assess the spatial distribution of photoconverted synaptic vesicles with EM (Rizzoli and Betz, 2004). Another method is based on the use of phlourins, 
$\mathrm{pH}$-sensitive fluorophores coupled to synaptic vesicle proteins, which become excitable and emit light upon vesicle fusion and quenched upon re-acidification of the synaptic vesicle (Ariel and Ryan, 2010). This technique enables monitoring synaptic vesicle fusion live and therefore offers good temporal resolution.

Presynaptic capacitance measurements are a direct method to monitor the fusion of vesicles with the plasma membrane, however, they are only applicable for the study of large synaptic boutons (Neher and Marty, 1982). Using this method, the change in membrane capacitance in response to strong stimulation paradigms is measured and directly related to the addition of membrane to the presynaptic terminal via synaptic vesicle fusion or removal of membrane through endocytosis. Although this approach has extremely high temporal resolution, it cannot distinguish between exo- or endocytosis-mediated membrane capacitance changes during stimulation or the morphological nature of the membrane trafficking events. Moreover, in large terminals harboring multiple active zone release sites (i.e. hippocampal mossy fiber boutons) whole-bouton capacitance changes are insensitive at the level of individual active zone release sites.

A common method used to measure the RRP in mixed neuron cultures is by perfusing hypertonic sucrose solution over the cell and recording the postsynaptic response (Rosenmund and Stevens, 1996). This technique is especially powerful in combination with low-density neuron culture systems, in which neurons form synapses onto themselves (autapses), because these autaptic cultures allow a very standardized experimental system to study the molecular mechanisms underlying synaptic function in individual neurons (Bekkers and Stevens, 1991). Hypertonic sucrose solutions cause the fusion of synaptic vesicles with the plasma membrane, which is believed to be independent of calcium influx and potentially caused by shrinkage of the presynaptic bouton. However, the exact mechanism of hypertonic sucrose-induced synaptic vesicle fusion is poorly understood.

The aforementioned techniques to determine RRP content in neurons require a degree of evoked fusion of synaptic vesicles. Factors such as calcium-dependent priming, short-term plasticity, and postsynaptic receptor saturation and sensitization can influence the RRP estimate. With HPF, freeze substitution, and electron tomography, I was able to characterize morphologically docked synaptic vesicles from synapses at rest. 


\subsection{Morphological correlates of mossy fiber facilitation}

Release probability and short-term plasticity are dependent upon multiple factors, including calcium channel-sensor coupling distance (Vyleta and Jonas, 2014), shape of the action potential (Geiger and Jonas, 2000), calcium buffers (Blatow et al., 2003; Dumas et al., 2004; Vyleta and Jonas, 2014), type of calcium sensor (Jackman et al., 2016), and accessibility to energy sources (i.e. mitochondria) (Kwon et al., 2016).

Despite comparable numbers of membrane-proximal synaptic vesicles within $40 \mathrm{~nm}$ of the active zone membrane, I found fewer morphologically docked and primed synaptic vesicles at individual mossy fiber active zones compared to Schaffer collateral synapses from the same slice. These data indicate that differences in the availability of docked and primed vesicles could, in addition to other factors, co-determine initial release probability. Past studies examined whether release probability is dependent on the availability of morphologically docked synaptic vesicles, however the conclusions did not lead to a general consensus (Eltes et al., 2017; Holderith et al., 2012; Millar et al., 2002; Schikorski and Stevens, 1997; XuFriedman et al., 2001). This question was worth revisiting in light of some limitations from past studies that can be circumvented with different techniques (i.e. cryo-fixation and electron tomography).

My findings are consistent with those reported by Eltes and colleagues that synapses with a low release probability (facilitating, tonic) harbor fewer docked synaptic vesicles than synapses with higher release probability (depressing, phasic) (Eltes et al., 2017). Along the same lines, Schikorski and Stevens found that the variability in the number of docked synaptic vesicles in the rat CA1 accounted for the heterogeneity in synaptic release probability and concluded that the number of docked vesicles contributes to synaptic release probability (Schikorski and Stevens, 1997). Conversely, my results differ from studies in the rodent cerebellum where synapses from parallel fibers (low release probability, facilitating) and synapses from climbing fibers (high release probability, depressing) harbored similar numbers of docked synaptic vesicles (Xu-Friedman et al., 2001). Further, in hippocampal associational/commissural synapses the number of docked synaptic vesicles and the release probability positively correlated with active zone area (Holderith et al., 2012). 
In contrast to Schaffer collateral synapses, mossy fiber synapses at rest had a second pool of membrane-proximal vesicles $5-20 \mathrm{~nm}$ from the active zone. I interpret the membraneproximal accumulation of vesicles in mossy fibers as structural evidence of a tethering step preceding synaptic vesicle docking/priming. Although undetected in wild-type Schaffer collaterals in the present study, the membrane-proximal accumulation of vesicles is highly reminiscent of the synaptic vesicle organization observed in Schaffer collateral synapses lacking key priming proteins, such as Munc13s, and core components of the neuronal SNARE complex, such as SNAP-25 and synaptobrevin-2 (Imig et al., 2014). This indicates that membrane-proximal vesicle tethering is not unique to mossy fibers per se, but implies that molecular processes responsible for forming the tethered pool operate differently in Schaffer collateral and mossy fiber synapses. Differences in molecular processes could involve a limited copy number of any of the aforementioned priming and SNARE proteins that, upon genetic deletion, manifest a membrane-proximal accumulation of synaptic vesicles. Specialized vesicle tethering mechanisms have evolved to support the transmitter release properties, and behaviors of distinct synapse types have been shown in specialized synapses such as invertebrate neuromuscular junctions and mammalian ribbon synapses (Hallermann and Silver, 2013).

My findings support a previously proposed vesicle tethering mechanism (Hallermann and Silver, 2013) for the first time in mossy fiber synapses. Synaptic vesicles are thought to loosely tether to the plasma membrane close enough for interaction to occur between v-SNAREs and t-SNARE (Neher and Brose, 2018). A tightening of the SNARE complex assembly results in morphological docking of synaptic vesicles to the plasma membrane mediated by Munc13 and CAPS priming molecules (Neher and Brose, 2018). These steps represent a loose and then tight synaptic vesicle docking state (Neher and Brose, 2018). The generation of a LS synaptic vesicle pool has been shown in dissociated mouse hippocampal neurons to rely on the calcium sensor synaptotagmin-1 (Chang et al., 2018). My finding of a membrane proximal pool of synaptic vesicles in mossy fiber synapses indicates the morphological correlate of tethered and therefore LS synaptic vesicles. The relationship between these tethered vesicles and the hypothesized LS state in tonic mossy fiber synapses requires further investigation, both in terms of its reliance of synaptotagmin-1, and in terms of how quickly the tethered pool is formed or depleted. These questions could be addressed by performing flash-and-freeze 
experiments-coupled with 3D ultrastructural analysis in synaptotagmin-1 mutant hippocampal slice cultures analogous to the study in dissociated neuron culture by Chang and colleagues (Chang et al., 2018).

Although I did not directly quantify filamentous material in my tomograms, the distance at which the membrane-proximal pool accumulated in wild-type mossy fiber synapses in the present study, and in priming-deficient Schaffer collateral synapses (Imig et al., 2014) is highly comparable to the length of long, single tethers described in cryo-electron tomographic reconstructions of frozen-hydrated synaptosomes (Fernández-Busnadiego et al., 2010, 2013). Since the molecular identity of vesicle tethers remains to be clarified, it is difficult to speculate which proteins are specifically responsible for generating a prominent membrane-proximal pool in wild-type mossy fibers at rest. Whereas synaptosomes isolated from RIM1 $\alpha$-deficient mice exhibited a perturbation of filamentous active zone material, it is unlikely that tethering is mediated by RIM1 $\alpha$ alone. Another candidate is bassoon, an active zone protein that has been implicated in rapid RRP replenishment in the cerebellum during high synaptic activity (Hallermann et al., 2010) and is necessary for the proper maturation of active zones in hippocampal mossy fiber synapses (Lanore et al., 2010).

I hypothesize that the tethered vesicle pool I observed at mossy fiber active zones is ideally situated to resupply the docked and primed pool of vesicles during sustained activity. Moreover, I propose that a low ratio of docked to tethered vesicles, as I observed in hippocampal mossy fibers, may serve as a structural feature distinguishing facilitating ("tonic") synapses. This view is compatible with recently proposed theories postulating that "tonic" and "phasic" synapses have different ratios of tightly (TS) and loosely (LS) docked vesicle states (Neher and Brose, 2018). In the future, additional experiments examining the time course of docked and tethered pool depletion during induced short- and long-term plasticity regimes are necessary to test these hypotheses.

A direct visualization and quantification of mossy fiber tethers by cryo-electron tomography would be informative, particularly in combination with genetic perturbations. Nevertheless, to perform such experiments in a tissue context would be exceptionally technically challenging, requiring a combination of HPF and the subsequent generation of lift-out FIB lamella and ultimately tomographically reconstructed under cryo conditions (Mahamid et al., 2015; Schaffer et al., 2019). Additional technical challenges would need to be addressed, 
including how to identify mossy fiber boutons in the vitrified sample, and how to identify active zones in the ideal orientation for tomography. 


\subsection{Synaptic vesicles, giant vesicles, and dense core vesicles at mossy fiber active zones}

Consistent with previous publications, my data revealed considerable morphological heterogeneity in the presynaptic vesicle pools at mossy fiber active zones, which comprised clear core synaptic vesicles (diameter range 38-59 nm), giant clear core vesicles, (diameter range 60-120 nm), and DCVs (diameter range 45-100 nm). However, my data represent the first systematic morphometric analysis of their dimensions and relative spatial distributions with respect to active zone release sites. Moreover, I demonstrate for the first time clear ultrastructural evidence that all three vesicle types dock in direct physical contact with the active zone membrane in a Munc13-dependent manner.

\subsubsection{Giant vesicles}

Although the presence of giant vesicles in mossy fiber boutons has been described (Henze et al., 2002; Laatsch and Cowan, 1966; Rollenhagen et al., 2007), the origin, cargo, and functional implications of this fascinating organelle remain enigmatic. Several lines of evidence support the hypothesis that giant vesicles containing glutamate neurotransmitter cargo contribute to glutamatergic signaling: i) electron micrographs revealed giant vesicles in proximity to mossy fiber active zones (Henze et al., 2002b; Laatsch and Cowan, 1966; Rollenhagen et al., 2007); ii) large amplitude "giant" mEPSCs recorded from postsynaptic CA3 pyramidal neurons were demonstrated to be monoquantal (Henze et al., 2002b); iii) gamma-radiation lesions ablating the DG abolished giant mEPSCs recorded from CA3 pyramidal neurons (Henze et al., 1997). My data expands upon these studies by demonstrating that the proportion of docked giant vesicles is highly comparable to the proportion of giant mEPSCs recorded from pyramidal cells. Although direct evidence that giant vesicles contain glutamate, or possess vesicular glutamate transporters, is still lacking. However, my observation that giant vesicle docking is abolished by deletion of Munc13 priming proteins indicates that they likely harbor at least some of the vesicular molecules required for evoked fusion.

In the scenario that if giant vesicles, though capable of docking, were actually incapable of fusing at the active zone membrane, it is conceivable that they limit access of "normal" synaptic vesicles to release sites. This hypothesis is not supported by my analyses, which failed to detect a correlation between docked vesicle numbers and the proportion of giant vesicles (data not shown). 
Multiple questions remain to be answered. Do giant vesicles fuse? Do they have the same vesicular release probability as a "normal" synaptic vesicle? Where and how are giant vesicles generated? Are these organelles unique to mossy fiber synapses? Several forms of activitydependent membrane retrieval operate in synapses that could generate large vesicular structures, including compound fusion (He et al., 2009), ultrafast endocytosis (Delvendahl et al., 2016; Watanabe et al., 2013b, 2013a), and bulk membrane retrieval (Cousin, 2009). Indeed, in the calyx of Held, large neurotransmitter-filled giant vesicles have been shown to result from activity-induced compound fusion of synaptic vesicles (He et al., 2009). Ultrafast endocytosis mechanisms generate large spherical endocytic intermediates in response to action potential-evoked release from small glutamatergic synapses from cultured hippocampal neurons (Watanabe et al., 2013a). More recently, functional analyses of endocytosis kinetics indicate that ultrafast modes of endocytosis also operate in other central synapses (Brockmann and Rosenmund, 2016; Delvendahl et al., 2016). Bulk membrane retrieval has only been observed in synapses during high activity states (Cousin, 2009) and is unlikely to be the origin of giant vesicles in mossy fiber synapses. It is also possible that giant vesicles result from de-granulation DCVs in a process comparable to that described for large DCVs in chromaffin cells (Shin et al., 2018). Although I occasionally observed filamentous electron dense material in the lumen of giant vesicles in the mossy fiber synapse, giant vesicles in mossy fibers persisted following pharmacological or genetic silencing in Munc13deletion mutants. Since both synaptic and DCV fusion at synaptic active zones is severely decreased upon loss of Munc13 priming proteins (Augustin et al., 1999; van de Bospoort et al., 2012; Varoqueaux et al., 2002), my data indicate that at least a considerable subpopulation of giant vesicles are generated via activity-independent mechanisms. Nevertheless, further analyses of ultrastructural changes induced during defined activity states are required to establish the contribution of compensatory endocytosis to large vesicle pools in mossy fiber synapses.

Alternative options include a form of constitutive membrane retrieval, which operates preferentially in mossy fiber synapses, or anterograde axonal trafficking of giant vesicles from the soma. I found evidence of giant vesicles in granule cell axonal projections in the CA3 from acute slice preparations alongside the other vesicle types, synaptic vesicles, and DCVs. This could indicate anterograde trafficking from the soma, however the transport direction of 
these vesicles in plastic embedded samples cannot be determined. Moreover, the purpose of these precursor giant vesicles and whether they are molecularly equipped to contribute to synaptic transmission is unclear. If a proportion of giant vesicles are morphological correlates of membrane retrieval, adaptor protein-3, an endocytosis-associated protein, would be one molecular candidate to further investigate this line of inquiry as it has a specific influence on mossy fiber endocytosis and synaptic vesicle dynamics (Evstratova et al., 2014; Scheuber et al., 2006).

The release of large quanta from giant vesicles has potential implications for mossy fiber function and several important questions remain open. For example, do giant vesicles fuse with the same release probability as other synaptic vesicles? Does quantal release from giant vesicles contribute to mossy fiber facilitation? These could be addressed by measuring mEPSCs in CA3 pyramidal neurons with increasing external calcium concentrations to determine whether the spontaneous fusion of giant vesicles changes in a calcium-dependent manner. In a previous study, the frequency of giant monoquantal events on CA3 pyramidal neurons did not increase in a calcium-dependent manner (Henze et al., 2002b). It has been further shown that vesicles with high membrane curvature, such as small synaptic vesicles, promote more lipid mixing than low-curvature vesicles, such as giant vesicles (Malinin et al., 2002). These findings indicate al ready that giant vesicles intrinsically have a lower fusogenicity than small synaptic vesicles.

Future directions to explore in terms of giant vesicles would include determining what proportion of giant vesicles represent endocytic intermediates generated by constitutive pathways. This question can be approached in several ways: (i) uptake assays using cellimpermeable fluorescent dyes, photoconvertable dyes, or electron dense particles; (ii) inhibition of endocytosis using pharmacological application of dynamin inhibitors; and (iii) changes in giant vesicle abundance/morphological characteristics in response to defined synaptic activity regimes.

In the case of uptake assays, ferritin (Farrant, 1954; Watanabe et al., 2013a), phluorins (Ariel and Ryan, 2010), and FM1-43 dyes (Branco et al., 2010; Rizzoli and Betz, 2004) have been used to investigate endocytosis or endocytic by-products in synapses by being captured in the membrane invagination during endocytosis. These methods are usually ideal for cell 
monolayers as is the case in autapses and dissociated hippocampal neuron culture (Ariel and Ryan, 2010; Branco et al., 2010; Watanabe et al., 2013a).

In the case of inhibiting endocytosis, DYNAsore and DYNgo are inhibitors of dynamin, a molecule necessary for pinching endocytosed vesicles to detach them from the presynaptic membrane (Daniel et al., 2012; Mccluskey et al., 2013). The relative abundance of giant vesicle-sized invaginations could be compared to the relative abundance of giant vesicles within a defined distance from the active zone. Such an experiment could also provide the locations of endocytosis in the synapse, though previous studies in cultured hippocampal neurons have demonstrated that ultrafast endocytosis occurs at peri-synaptic sites, at the periphery of the active zone (Watanabe et al., 2013a). One caveat to dynamin inhibition in slice cultures is that dynamin has a role in other membrane trafficking events within the entire cell, and cytotoxicity of the dynamin inhibitors could partially occlude the findings (McMahon and Boucrot, 2011).

\subsubsection{Pharmacological enhancement of release probability}

Mossy fiber release probability is sensitive to changes in presynaptic CAMP concentration (Fykse et al., 1995; Steuer Costa et al., 2017; Weisskopf et al., 1994). As previously mentioned, I applied forskolin to cultured hippocampal slices to enhance mossy fiber release probability by initiating a signaling cascade involving forskolin-mediated activation of AC1, increased intracellular production of CAMP, and activation of PKA. Forskolin-mediated elevations of CAMP and activation of PKA are used to induce a chemical form of LTP (Fernandes et al., 2015; Huang et al., 1994a; Villacres et al., 1998; Weisskopf et al., 1994). My analyses of forskolinpotentiated mossy fiber boutons reveal only a mild increase in the number of docked synaptic vesicles at the active zone membrane. These findings are in line with previous findings in mossy fiber synapses that forskolin-induced enhancement of synaptic transmission has little effect on the functional RRP measured via electrophysiological approaches (Midorikawa and Sakaba, 2017). These findings indicate that the downstream target of PKA likely acts at a post docking and priming step. Multiple downstream phosphorylation targets of PKA have been identified that also contribute to the regulation of the vesicular release machinery. These include complexins (Cho et al., 2015), rab3A (Castillo et al., 1997; Geppert et al., 1997), and synaptotagmin-12 (Kaeser-Woo et al., 2013). 
Complexins are a molecular candidate implicated in mossy fiber LTP (Gibson et al., 2005). Cho and colleagues demonstrated that complexin is phosphorylated by PKA and increases spontaneous synaptic vesicle fusion after tetanus stimulation in the Drosophila neuromuscular junction (Cho et al., 2015). Although genetic deletion of complexin-2 caused a reduction in mossy fiber LTP, forskolin-induced potentiation was unaffected (Gibson et al., 2005). Previous ultrastructural analyses in Schaffer collateral synapses from complexin-1, -2, and -3 triple knock-out support the idea that complexin acts on vesicular release machinery downstream of synaptic vesicle docking (Imig et al., 2014).

In mossy fiber synapses, rab3A is not essential for paired-pulse facilitation, a form of shortterm plasticity (Castillo et al., 1997). However, genetic deletion of rab3A from mossy fiber synapses ablated mossy fiber LTP (Castillo et al., 1997). Conversely, forskolin-induced mossy fiber potentiation was intact in rab3A knock-out animals (Castillo et al., 1997). Rab3a is not directly phosphorylated by PKA but has strong interaction with two PKA targets at the presynapse, RIM and rabphillin (Castillo et al., 1997; Fykse et al., 1995). Castillo and colleagues propose that rab3a is an effector downstream of PKA and, due to interactions with RIM and rabphillin, has a role in controlling the calcium influx during LTP (Castillo et al., 1997).

Synaptotagmin-12 is phosphorylated by PKA and co-localizes with synaptotagmin-1 on synaptic vesicles in mossy fiber synapses (Kaeser-Woo et al., 2013; Maximov et al., 2007). Although synaptoagmin-12 is not a calcium-sensing synaptotagmin, it is activated via cAMP (Kaeser-Woo et al., 2013; Pang and Südhof, 2010). Synaptotagmin-12 interacts with synaptotagmin-1, inhibiting its function as a calcium sensor, however once phosphorylated by PKA, synaptotagmin-12 dissociates from synaptotagmin-1 thus increasing vesicular release probability (Kaeser-Woo et al., 2013; Maximov et al., 2007).

PKA phosphorylation can also inhibit synaptic proteins, as in the case of tomosyn. Tomosyn prevents SNARE complex assembly by binding syntaxin (Baba et al., 2005). Phosphorylation of tomosyn diminishes its interaction with syntaxin (Baba et al., 2005; Ben-Simon et al., 2015; Fujita et al., 1998; Hatsuzawa et al., 2003), making more syntaxin available for SNARE complex formation at the active zone membrane. Genetic knockdown of tomosyn increases release probability in hippocampal mossy fiber synapses, causing changes to short-term plasticity and occluding LTP (Ben-Simon et al., 2015). 
In summary, presynaptic cAMP signaling leads to enhanced synaptic transmission from mossy fiber synapses (Huang et al., 1994a; Villacres et al., 1998; Weisskopf et al., 1994). Pharmacological application of forskolin works on this signaling pathway by enhancing presynaptic CAMP. I tested whether increasing synaptic efficacy at mossy fiber synapses in slice culture increase the number of docked vesicles. However, no differences were observed between forskolin and control or with DCG-VI-treated slices. My findings support the hypothesis that the enhancement of synaptic transmission by CAMP signaling pathways works downstream of synaptic vesicle docking and priming.

\subsubsection{DCVs in mossy fiber synapses}

The present study found that DCVs dock directly at the active zone in mossy fiber synapses at rest in acute and slice culture preparations. This finding is in contrast to analogous studies in Schaffer collateral synapses from past and present studies (van de Bospoort et al., 2012; Farina et al., 2015; Imig et al., 2014; Siksou et al., 2009a). In the absence of Munc13 priming proteins, DCV docking, as well as all vesicle docking, at mossy fiber active zones was completely abolished and a 2.5-fold increase in DCV abundance was observed within $100 \mathrm{~nm}$ of the active zone membrane. Although large DCV docking was unaffected by Munc13deletion in chromaffin cells (Man et al., 2015), these data are consistent with the finding that Munc13-1 is required for synaptic DCV exocytosis in mammalian neuron cultures (van de Bospoort et al., 2012).

While strong stimulation is necessary to trigger DCV release in cultured neurons (van de Bospoort et al., 2012), I found that mossy fiber synapses at rest harbor docked DCVs and, in the absence of Munc13 priming proteins, DCVs accumulated at the active zone which could indicate that DCV fusion (i.e. neuropeptide release) at mossy fiber synapses occurs to some degree at a basal level. Accordingly, bassoon mutant mice exhibit an accumulation of BDNFand enkephalin-containing DCVs in the presynaptic terminals of mossy fiber synapses (Dieni et al., 2012, 2015), concurrently, in dissociated mouse hippocampal neurons, bassoon mutants exhibit a reduction in the number of fusion-competent vesicles (Altrock et al., 2003). Both enkephalin and BDNF are involved in synaptic plasticity of the mossy fiber-CA3 synapse (Derrick et al., 1992; Dieni et al., 2012; Li et al., 2010). BDNF contributes to mossy fiber potentiation in a transactivation mechanism that leads to increased synaptic transmission, however the retrograde signaling molecules are still unknown (Huang et al., 2008). Another 
neuropeptide, enkephalin, binds presynaptic $\mu$-opioid receptors and is involved in frequencydependent enhancement of mossy fiber-CA3 synaptic transmission during LTP (Derrick et al., 1992). Taken together, these data indicate that synaptic vesicles and neuropeptidecontaining DCVs share overlapping molecular mechanisms that operate in steps preceding fusion at mossy fiber active zones.

Alternatively, the accumulation of DCVs may reflect a homeostatic mechanism triggered in response to the loss of network activity in Munc13-deficient slices. In support of this hypothesis, chronic pharmacological silencing of cultured mammalian neurons treated for 48 hours with TTX led to an accumulation of DCVs in both inhibitory and excitatory synapses (Tao et al., 2018a). Furthermore, Tao and colleagues found more membrane-proximal DCVs after chronic TTX treatment (Tao et al., 2018a). Additionally, the researchers found evidence of DCV fusion at the active zone as well as at non-synaptic sites following 48 hours of TTX treatment (Tao et al., 2018a). Tao and colleagues postulated that DCV accumulation in chronically silenced neurons is involved in homeostatic metaplasticity by transporting active zone material to the presynaptic membrane (Tao et al., 2018a). Homeostatic metaplasticity is the compensatory mechanism of a neuron to enhance or diminish synaptic plasticity (i.e. LTP or long-term depression) (Abraham, 2008). In this case, Tao and colleagues propose that additional active zone material is trafficked to chronically silenced synapses to increase the active zone scaffolding material and thus increase the number of release sites at the synapse as has been previously described (Shapira et al., 2003; Sorra et al., 2006; Tao et al., 2018a). However, the comparable size of active zones reconstructed in Munc13-deficent and control mossy fiber synapses in the present study, and the lack of DCV accumulation in Munc13deficient Schaffer collateral synapses (Imig et al., 2014) appear inconsistent with this notion. To address this, future studies should investigate whether BDNF- or enkephalin-positive DCVs accumulate, whether the DCVs contain active zone molecules such as piccolo or bassoon (Maas et al., 2012; Shapira et al., 2003; Tao-Cheng, 2007), or whether active zones areas increase in mossy fiber synapses in Munc13-deficient slices.

My findings indicate Munc13s facilitate DCV docking and priming at mossy fiber active zones. Past studies show that Munc13-1 is necessary for mossy fiber LTP (Yang and Calakos, 2011). Neuropeptides released from mossy fiber boutons modulate synaptic transmission by modulation of pre- and postsynaptic targets (Chavkin et al., 1983; McQuiston and Colmers, 
1996; Salin et al., 1995; Sherwood and Lo, 1999; Weisskopf et al., 1993). I speculate that DCV fusion mediated by Munc13s modulate synaptic transmission in mossy fiber boutons, a potentially novel mechanism for Munc13s in LTP. Further experimentation is needed to better understand the role of Munc13s in neuropeptide signaling in mossy fiber synapses. Furthermore, I postulate that DCVs likely undergo a basal level of fusion at rest based on the accumulation of DCVs in Munc13-deficient mossy fiber synapses. However, extensive experimentation would be necessary to test whether DCVs fuse in mossy fiber boutons under basal conditions. The speculations could be tested along similar lines as DCV fusion experimentation previously performed in dissociated hippocampal cultures (van de Bospoort et al., 2012; Farina et al., 2015), however be carried out in organotypic slice cultures. By using genetic targeting of granule cells (Kohara et al., 2014), coupled with optogenetic stimulation (Madisen et al., 2012), and phluorin-labeled DCVs (van de Bospoort et al., 2012) one could explore the parameters necessary to evoke DCV fusion in mossy fiber boutons and start examining the molecular machinery regulating synaptic release.

DCVs in mossy fiber synapses have varying morphological characteristics; some DCVs have diameters similar to those of synaptic vesicles, the electron dense material in the center is sometimes segregated to the center of the vesicle with varying halo sizes between the vesicle membrane and the electron dense core, and at times the core is eccentrically located within the DCV lumen. It is possible that the differences in size and dense core opacity are caused by piecemeal degranulation of individual DCVs, as has been described in mouse mast and chromaffin cells, as well as thalamic and hypothalamic neurons (Crivellato et al., 2005). The neuropeptide content of each DCV is not known in mossy fiber boutons. It is also unknown if there is an ultrastructural correlate to neuropeptide content in individual DCVs; if for example, BDNF could be contained in DCVs with a halo, whereas enkephalin or dynorphin are packaged in DCVs with no halo. Both morphologies were observed in mossy fiber boutons. Further work is needed to correlate the morphological characteristics of DCVs in mossy fiber synapses and the neuropeptide content stored within each DCV. If there is a correlation between DCV morphology and neuropeptide content, one could make further comparisons of DCV accumulation at mossy fiber active zones and functional aspects of the synapse. 


\subsubsection{Presynaptic CAMP and DCV docking}

In the present study, I applied forskolin to hippocampal slice cultures to pharmacologically enhance release probability in mossy fiber synapses. Forskolin activates AC1, which in turn increases presynaptic CAMP production and enhances synaptic transmission via downstream effectors (Trudeau et al., 1996; Villacres et al., 1998; Weisskopf et al., 1994). After acute treatment with forskolin, I found an increase in DCV docking, however no change in the spatial density of DCVs within $40 \mathrm{~nm}$ of the active zone membrane, indicating a cAMP-dependent redistribution of membrane proximal DCVs. Previous work in Caenorhabditis elegans demonstrated that elevating presynaptic cAMP levels with a bacterial light-activated adenylate cyclase increased DCV fusion at the neuromuscular junction (Steuer Costa et al., 2017). Work from the present study supports the idea that DCV docking is influenced by forskolin-induced production of presynaptic cAMP in the mossy fiber synapse but direct evidence of DCV fusion is lacking. Verhage and colleagues showed that DCV exocytosis from dissociated rodent hippocampal neurons requires a large, global increase of calcium ions in the presynaptic bouton that occurs with persistent opening of presynaptic calcium channels during high synaptic activity (Verhage et al., 1991). It is possible that the increase I observed in membrane-docked DCVs is due to downstream effectors of presynaptic cAMP acting independently of action-potential driven changes in presynaptic calcium.

The cAMP signaling pathway has been extensively studied in many cell types including neurons (Seino and Shibasaki, 2005). Two major downstream targets of cAMP in neurons are PKA and Epacs (Evans and Morgan, 2003; Robichaux and Cheng, 2018; Shibasaki et al., 2007). Activation of PKA and Epac depends on the intracellular concentration of CAMP; PKA has a relatively high affinity for CAMP and requires low nanomolar concentrations, whereas Epac binds to CAMP with lower affinity at concentrations at which PKA is saturated (Seino and Shibasaki, 2005). No studies have explored the effects of PKA or Epac on DCV fusion in neurons, however several studies have shown effects of PKA and Epac on secreting cells. Epac, but not PKA, has been implicated in increased secretion of insulin-containing granules from pancreatic beta cells in human tissue (Kang et al., 2003). In mice, PKA-dependent and independent mechanisms of cAMP-induced insulin secretion from beta cells have been described (Eliasson et al., 2003). In this context, to dissect which pathway, PKA- or Epac- 
dependent, is involved in the forskolin-induced increase in DCV docking could provide insight into the signaling cascades required for DCV fusion in mossy fiber synapses.

In summary, forskolin-induced presynaptic cAMP in hippocampal mossy fiber boutons changes the membrane-proximal distribution of DCVs; increased CAMP leads to increased DCV docking and DCG-IV-induced reduction in CAMP results in fewer docked DCVs. Presynaptic CAMP is part of the pathway utilized to enhance synaptic transmission in mossy fiber synapses during sustained activity. Neuropeptides, packaged in DCVs, are also implicated in modulating mossy fiber synaptic transmission. This CAMP-induced phenomenon could be a novel mechanism for DCV docking in hippocampal mossy fiber synapses. 


\subsection{Development of Schaffer collateral and mossy fiber synapses in slice culture}

Synapse development is incomplete at birth. Indeed, structural and functional changes occur in animals such as rodents throughout the life time of the animal. In the present study, I investigated the ultrastructure of mossy fiber and Schaffer collateral synapses at two developmental time points (DIV14 and DIV28) in the mouse hippocampus. My data demonstrate a developmental increase in the spatial density of docked synaptic vesicles at both Schaffer collateral and mossy fiber active zones between DIV14 and DIV28.

Mossy fiber and Schaffer collateral synapses undergo structural and functional maturation between the ages of P3 and P21 (Amaral and Dent, 1981; Battistin and Cherubini, 1994; Buchs et al., 1993; Helassa et al., 2018; Ho et al., 2007; Hussain and Carpenter, 2001; LaVail and Wolf, 1973; Marchal and Mulle, 2004; Mori-Kawakami et al., 2003; Münster-Wandowski et al., 2013; Rose et al., 2013; Schmitz et al., 2003; De Simoni et al., 2003; Wilke et al., 2013). Structural maturation of mossy fiber boutons in cultured slices closely resembles the time course described in vivo (Amaral and Dent, 1981; Galimberti et al., 2006; Wilke et al., 2013). Since previous studies have concluded that presynaptic structural maturation of mossy fiber boutons peaks at P14, with only minor changes beyond that, including a pruning of filopodial extensions and an increase in bouton volume (Amaral and Dent, 1981; Galimberti et al., 2006; Wilke et al., 2013), my observations yield novel insight into mossy fiber synapse development at the level of fine structural organization at the active zone. Synaptogenesis and spine maturation in CA1 pyramidal neurons has been described (Fiala et al., 1998; Harris and Weinberg, 2012; Harris et al., 1992). Schaffer collateral spines undergo structural maturation from P1 to P40 in both the proportion of spine morphologies and presence of specialized organelles such as spine apparatuses (Fiala et al., 1998; Harris and Weinberg, 2012; Harris et al., 1992; De Simoni et al., 2003). By P12 in rats, most excitatory synapses in the stratum lucidum of the CA1 are located at either the dendritic shaft, or the tip of postsynaptic spines (Fiala et al., 1998). Further, from P15 to adult (P70) in the rat hippocampus, spine morphologies change in their relative proportion and density (Harris et al., 1992). Harris and colleagues found a higher proportion of thin spines and, inversely, a lower proportion of stubby spines in the CA1 at P70 compared to P15 in rats (Harris et al., 1992). The 
heterogeneity of spine morphologies in the CA1 has been postulated to influence structural plasticity during high-activity states, however this remains an open question (Harris and Weinberg, 2012).

The developmental increase in the spatial density of docked synaptic vesicles in Schaffer collateral and mossy fiber synapses was not paralleled by corresponding changes in the efficacy of neurotransmitter release as measured by patch clamp electrophysiology. Neither mean evoked EPSC amplitudes nor paired-pulse ratios changed significantly during this developmental window. This observation was not entirely unexpected, since unlike structural development, the functional changes in both Schaffer collateral and mossy fiber synapses observed in past studies occurs mostly before P14 and after P28 in vivo (Battistin and Cherubini, 1994; Ho et al., 2007; Hussain and Carpenter, 2001; Marchal and Mulle, 2004; Mori-Kawakami et al., 2003; Rose et al., 2013; Schiess et al., 2010; Schmitz et al., 2003). In acute rat slices, mossy fiber synapses exhibit similar paired-pulse facilitation and post-tetanic potentiation between P10 and P20 (Hussain and Carpenter, 2001). However, between P14 and P21 mossy fiber synapses in acute mouse slices exhibit increased frequency facilitation that is not dependent on postsynaptic receptors (Marchal and Mulle, 2004). Developmental changes in Schaffer collateral synaptic function in acute slices also occur before P14 and after P28 in rodents (Hussain and Carpenter, 2001; Schiess et al., 2010).

Understanding why the observed increase in the spatial density of docked synaptic vesicles does not translate to detectable changes in postsynaptic responses requires the consideration of the complex interplay between multiple factors. These factors include presynaptic calcium channels and buffers, as well as developmental structural and molecular changes in the postsynaptic compartment, and in the abundance and properties of postsynaptic transmitter receptors.

Modifications to presynaptic calcium channels such as the relative proportion of VGCC subtype can shape presynaptic function. Spontaneous and evoked synaptic currents increase during development in dissociated hippocampal neurons (Basarsky et al., 1994). Dissociated cultured neurons had a developmental increase in calcium influx that coincided with an increase of the proportion of $P / Q$ calcium channel expression as the neurons matured (Basarsky et al., 1994). At mossy fiber synapses (at P20-23), the number of P/Q-type calcium 
channels estimated per active zone is approximately 23 (Vyleta and Jonas, 2014). It is, however, not known how this number is developmentally regulated.

Developmental changes in the spatial distribution of presynaptic calcium channels have been described in the rat cerebellum (Miki et al., 2017). Between P14 and P28, calcium channels decrease in number and form organized clusters that preferentially aggregate at the periphery of the active zone (Miki et al., 2017). Consequently, Miki and colleagues found that the number of docked vesicles correlated with the number of calcium channel clusters indicating fewer vesicles were docked at active zones at P28 (Miki et al., 2017).

Bornschein and colleagues found that there was a developmental decrease in the calcium channel-sensor coupling distance in somatosensory layer-V pyramidal neurons from wildtype mice between the ages of P10 and P24 (Bornschein et al., 2019). Despite tighter coupling, there was no change in the RRP and release probability between the two ages (Bornschein et al., 2019), indicating that developmental changes can occur without affecting the functional output of a given synapse. In mossy fiber synapses, calcium channel-synaptic vesicle coupling distance is around $70 \mathrm{~nm}$, much larger than other synapses with a higher release probability ( $20 \mathrm{~nm}$ in the Calyx of Held) (Chen et al., 2015). An unanswered question is whether mossy fiber or Schaffer collateral synapses have a developmental change in calcium channel-sensor coupling distance.

The unchanged functional output of both mossy fiber and Schaffer collateral synapses could also be due to an increase in endogenous calcium buffers (Blatow et al., 2003; Luiten et al., 1994). For example, calbindin is a fast-acting endogenous calcium buffer that influences synaptic functional properties in mossy fiber synapses (Blatow et al., 2003; Dumas et al., 2004). In the developing rat hippocampus, calbindin expression steadily increases in granule cells and mossy fiber synapses from P5 to P20 (Luiten et al., 1994).

Presynaptic mitochondria can rapidly sequester presynaptic calcium in synapses to maintain a low release probability (Kwon et al., 2016). Presynaptic mitochondria are more abundant in mossy fiber boutons compared to Schaffer collateral synapses (Amaral and Dent, 1981; Shepherd and Harris, 1998; Smith et al., 2016). Indeed, as assessed by 2D transmission EM, in the untreated slice cultures at DIV28, nearly every mossy fiber bouton contained at least one mitochondrion whereas approximately one third of Schaffer collateral synapses harbored a 
presynaptic mitochondrion (see Figure 34 in the appendix). However, developmental changes in the presence of presynaptic mitochondria are poorly understood.

Postsynaptic development includes changes in glutamate receptor subtype expression, and in the localization of receptors in relation to synaptic release sites (Ho et al., 2007; Marchal and Mulle, 2004; Sans et al., 1996). For example, CA3 pyramidal neurons undergo a developmental increase in kanaite receptor expression at the mossy fiber-CA3 postsynaptic compartment that marks the onset of frequency facilitation and potentiation observed at these synapses (Marchal and Mulle, 2004). In another study, expression of calciumpermeable AMPA receptors underlies early post-natal long-term depression exhibited at mossy fiber-CA3 synapses (Ho et al., 2007). In Schaffer collateral synapses, Sans and colleagues found an increase in the expression of proteins associated with the postsynaptic density involved in anchoring NMDA receptors at the synaptic junction (Sans et al., 1996). They postulated that during development, the accumulation of NMDA-anchoring molecules helps localize NMDA receptors closer to release sites (Sans et al., 1996).

The factors outlined above, perhaps in combination, may occlude the detection of functional changes associated with my observed developmental changes in vesicle docking. As described in other synapses, the structural alteration in presynaptic vesicle organization may be silent, exerting no influence on release probability or on short-term facilitation (Bornschein et al., 2019). 


\section{Conclusion}

In the present study, I performed a comparative ultrastructural analysis of the functionally distinct hippocampal Schaffer collateral and mossy fiber synapses to assess whether the availability of docked and primed synaptic vesicles contributes to differences in release probability. To address this question, I combined hippocampal slice culture, HPF, AFS, and electron tomography to accurately resolve the organization of vesicles at presynaptic active zones.

My ultrastructural analyses revealed mossy fiber synapses, which functionally exhibit a low release probability and robust synaptic facilitation, harbored fewer morphologically docked synaptic vesicles than Schaffer collateral synapses. This supports the hypothesis that differences in synaptic release probability depend on the availability of fusion-competent synaptic vesicles. Additionally, mossy fiber synapses had a prominent second, membrane proximal pool of vesicles that represent a LS state of tethered synaptic vesicles, which are ideally positioned to rapidly replenish the RRP during periods of increased synaptic activity. This membrane-proximal accumulation was not observed in Schaffer collateral synapses.

Additionally, my work identified, for the first time, that three morphologically distinct types of vesicles dock at mossy fiber active zones: synaptic vesicles, giant vesicles (clear-core vesicles with a diameter exceeding $60 \mathrm{~nm}$ ), and DCVs. All three vesicle types docked at mossy fiber synapses in a Munc13-dependent manner. Past studies reported the presence of giant vesicles and postulated they were neurotransmitter-filled vesicles responsible for giant monoquantal events observed in CA3 pyramidal neurons (Henze et al., 2002b; Laatsch and Cowan, 1966; Rollenhagen et al., 2007). I found that giant vesicles docked exclusively at mossy fiber active zones in the same proportion as giant mEPSC events of mossy fiber origin measured in slice culture. Giant vesicles were not merely by-products of endocytosis because they persisted in acute, pharmacological silencing of network activity and in constitutively silent, Munc13-deficient mossy fiber synapses. Evidence suggests that giant vesicles may be trafficked from granule cell somas, however many questions about giant vesicle identity and origin remain.

I performed a quantitative analysis of respective vesicle pools at mossy fiber active zones and compared my data with published functional estimates of the RRP. I demonstrated a 
considerable overlap between the total numbers of morphologically docked and functionally primed and fusion-competent synaptic vesicles and proposed that larger reported RRP estimates involved the recruitment of membrane-proximal vesicles.

I examined whether changes in synaptic release probability induced by acute pharmacological manipulations of presynaptic cAMP trigger corresponding changes in vesicle organization. My data reveal that DCV docking is particularly sensitive to changes in presynaptic cAMP levels. Surprisingly, I observed no change in the organization of docked synaptic or giant vesicles after increasing presynaptic cAMP. These findings support a view in which mechanisms mediating CAMP-dependent potentiation of mossy fiber synaptic transmission operate downstream of synaptic and giant vesicle docking. Additionally, they highlight the potential modulatory role of DCV-mediated neuropeptide release in mossy fiber plasticity processes.

In conclusion, my work further supports the hypothesis that initial release probability is determined by the availability of docked and primed vesicles and that the structural organization of vesicles at active zone release sites can provide insight into presynaptic functional properties. Furthermore, it emphasizes that methodical and thorough highresolution ultrastructural analyses are useful to reveal novel insight into ultrastructurefunction relationships in other synapse types in the brain. 


\section{Bibliography}

Abraham, W.C. (2008). Metaplasticity: Tuning synapses and networks for plasticity. Nat. Rev. Neurosci. 9, 387-399.

Acsády, L., Kamondi, A., Sík, A., Freund, T., and Buzsáki, G. (1998). GABAergic cells are the major postsynaptic targets of mossy fibers in the rat hippocampus. J. Neurosci. 18, 33863403.

Altrock, W.D., Tom Dieck, S., Sokolov, M., Meyer, A.C., Sigler, A., Brakebusch, C., Fässler, R., Richter, K., Boeckers, T.M., Potschka, H., et al. (2003). Functional inactivation of a fraction of excitatory synapses in mice deficient for the active zone protein bassoon. Neuron $37,787-$ 800 .

Amaral, D.G., and Dent, J.A. (1981). Development of the Mossy Fibers of the Dentate Gyrus : I . A Light and Electron Microscopic Study of the Mossy Fibers and Their Expansions. J. Comp. Neurol. 195, 51-86.

Amaral, D.G., Ishizuka, N., and Claiborne, B. (1990). Neurons, numbers and the hippocampal network. pp. 1-11.

Amaral, D.G., Scharfman, H.E., and Lavenex, P. (2007). The Dentate Gyrus: A Comprehensive Guide to Structure, Function, and Clinical Implications (Elsevier).

Andersen, P., Holmquist, B., and Voorhoeve, P.E. (1966). Entorhinal activation of dentate granule cells. Acta Physiol Scand 66, 448-460.

Andrews-Zwilling, Y.S., Kawabe, H., Reim, K., Varoqueaux, F., and Brose, N. (2006). Binding to Rab3A-interacting molecule RIM regulates the presynaptic recruitment of Munc13-1 and ubMunc13-2. J. Biol. Chem. 281, 19720-19731.

Aniksztejn, L., and Ben-Ari, Y. (1991). Novel form of long-term potentiation produced by a K+ channel blocker in the hippocampus. Nature 349, 67-69.

Arancillo, M., Min, S.W., Gerber, S., Münster-Wandowski, A., Wu, Y.J., Herman, M., Trimbuch, T., Rah, J.C., Ahnert-Hilger, G., Riedel, D., et al. (2013). Titration of Syntaxin1 in mammalian synapses reveals multiple roles in vesicle docking, priming, and release probability. J. Neurosci. 33, 16698-16714.

Aravamudan, B., Fergestad, T., Davis, W.S., Rodesch, C.K., and Broadie, K. (1999). Drosophila UNC-13 is essential for synaptic transmission. Nat. Neurosci. 2, 965-971.

Ariel, P., and Ryan, T.A. (2010). Optical mapping of release properties in synapses. Front. Neural Circuits 4, 1-10.

Ariel, P., Hoppa, M.B., and Ryan, T.A. (2013). Intrinsic variability in Pv, RRP size, $\mathrm{Ca}^{2+}$ channel repertoire, and presynaptic potentiation in individual synaptic boutons. Front. Synaptic Neurosci. 4, 1-18.

Atwood, H.L., and Jahromi, S.S. (1978). Fast-Axon Synapses of a Crab Leg Muscle. 9, 1-15.

Atwood, H.L., and Karunanithi, S. (2002). Diversification of synaptic strength: presynaptic elements. Nat. Rev. Neurosci. 3, 497-516.

Augustin, I., Rosenmund, C., Südhof, T.C., and Brose, N. (1999). Munc13-1 is essential for fusion competence of glutamatergic synaptic vesicles. Nature $400,457-461$. 
Baba, T., Sakisaka, T., Mochida, S., and Takai, Y. (2005). PKA-catalyzed phosphorylation of tomosyn and its implication in $\mathrm{Ca}^{2+}$-dependent exocytosis of neurotransmitter. J. Cell Biol. $170,1113-1125$.

Barbaresi, P., Gazzanelli, G., and Malatesta, M. (2001). p-Aminobutyric acid transporters in the cat periaqueductal gray: A light and electron microscopic immunocytochemical study. J. Comp. Neurol. 429, 337-354.

Barovsky, K., Pedone, C., and Brooker, G. (1984). Distinct mechanisms of forskolin-stimulated cyclic AMP accumulation and forskolin-potentiated hormone responses in $\mathrm{C} 6-2 \mathrm{~B}$ cells. Mol Pharmacol 25, 256-260.

Basarsky, T.A., Parpura, V., and Haydon, P.G. (1994). Hippocampal synaptogenesis in cell culture: developmental time course of synapse formation, calcium influx, and synaptic protein distribution. J. Neurosci. 14, 6402-6411.

Battistin, T., and Cherubini, E. (1994). Developmental Shift From Long-term Depression to Long-term Potentiation at the Mossy Fibre Synapses in the Rat Hippocampus. Eur. J. Neurosci. 6, 1750-1755.

Baumeister, W. (2002). Electron tomography: Towards visualizing the molecular organization of the cytoplasm. Curr. Opin. Struct. Biol. 12, 679-684.

Beaulieu, C., and Somogyi, P. (1990). Targets and Quantitative Distribution of GABAergic Synapses in the Visual Cortex of the Cat. Eur. J. Neurosci. 2, 296-303.

Bekkers, J.M., and Stevens, C.F. (1991). Excitatory and inhibitory autaptic currents in isolated hippocampal neurons maintained in cell culture. Proc. Natl. Acad. Sci. 88, 7834-7838.

Bekkers, J.M., and Stevens, C.F. (1995). Quantal analysis of EPSCs recorded from small numbers of synapses in hippocampal cultures. J. Neurophysiol. 73, 1145-1156.

Ben-Simon, Y., Rodenas-Ruano, A., Alvina, K., Lam, A.D., Stuenkel, E.L., Castillo, P.E., and Ashery, U. (2015). A Combined Optogenetic-Knockdown Strategy Reveals a Major Role of Tomosyn in Mossy Fiber Synaptic Plasticity Report A Combined Optogenetic-Knockdown Strategy Reveals a Major Role of Tomosyn in Mossy Fiber Synaptic Plasticity. Cell Rep. 12, 396-404.

Bennett, M.K., Calakos, N., and Scheller, R.H. (1992). Syntaxin: A synaptic protein implicated in docking of synaptic vesicles at presynaptic active zones. Science 257, 255-259.

Blackstad, T.W., and Kjaerheim, A. (1961). Special axo-dendritic synapses in the hippocampal cortex: Electron and light microscopic studies on the layer of mossy fibers. J. Comp. Neurol. $117,133-159$.

Blatow, M., Caputi, A., Burnashev, N., Monyer, H., and Rozov, A. (2003). Ca ${ }^{2+}$ buffer saturation underlies paired pulse facilitation in calbindin-D28k-containing terminals. Neuron $38,79-88$.

Böhme, M.A., Beis, C., Reddy-Alla, S., Reynolds, E., Mampell, M.M., Grasskamp, A.T., Lützkendorf, J., Bergeron, D.D., Driller, J.H., Babikir, H., et al. (2016). Active zone scaffolds differentially accumulate Unc13 isoforms to tune $\mathrm{Ca}^{2+}$ channel-vesicle coupling. Nat. Neurosci. 19, 1311-1320.

Borges-Merjane, C., Kim, O., and Jonas, P. (2020). Functional Electron Microscopy (“" Flash and Freeze "') of Identified Cortical Synapses in Acute Brain Slices NeuroResource Functional 
Electron Microscopy (" Flash and Freeze "') of Identified Cortical Synapses in Acute Brain Slices. Neuron 105, 1-15.

Bornschein, G., Eilers, J., and Schmidt, H. (2019). Neocortical High Probability Release Sites Are Formed by Distinct $\mathrm{Ca}^{2+}$ Channel-to-Release Sensor Topographies during Development. Cell Reports 28, 1410-1418.e4.

Borries, B. V., and Ruska, E. (1933). Die Abbildung durchstrahlter Folien im Elektronenmikroskop. Zeitschrift Für Phys. 83, 187-193.

van de Bospoort, R., Farina, M., Schmitz, S.K., de Jong, A., de Wit, H., Verhage, M., and Toonen, R.F. (2012). Munc13 controls the location and efficiency of dense-core vesicle release in neurons. J. Cell Biol. 199, 883-891.

Branco, T., Marra, V., and Staras, K. (2010). Examining size-strength relationships at hippocampal synapses using an ultrastructural measurement of synaptic release probability. J. Struct. Biol. 172, 203-210.

Brandt, D.S., Coffman, M.D., Falke, J.J., and Knight, J.D. (2012). Hydrophobic contributions to the membrane docking of synaptotagmin 7 C2A domain: Mechanistic contrast between isoforms 1 and 7. Biochemistry 51, 7654-7664.

Brandt, M.D., Jessberger, S., Steiner, B., Kronenberg, G., Reuter, K., Bick-Sander, A., Behrens, W. von der, and Kempermann, G. (2003). Transient calretinin expression defines early postmitotic step of neuronal differentiation in adult hippocampal neurogenesis of mice. Mol. Cell. Neurosci. 24, 603-613.

Breustedt, J., Gundlfinger, A., Varoqueaux, F., Reim, K., Brose, N., and Schmitz, D. (2010). Munc13-2 differentially affects hippocampal synaptic transmission and plasticity. Cereb. Cortex 20, 1109-1120.

Brockmann, M.M., and Rosenmund, C. (2016). Catching Up with Ultrafast Endocytosis. Neuron 90, 423-424.

Brockmann, M.M., Maglione, M., Willmes, C.G., Stumpf, A., Bouazza, B.A., Velasquez, L.M., Grauel, M.K., Beed, P., Lehmann, M., Gimber, N., et al. (2019). RIM-BP2 primes synaptic vesicles via recruitment of Munc13-1 at hippocampal mossy fiber synapses. Elife 8, 1-22.

Brodin, L., Bakeeva, L., and Shupliakov, O. (1999). Presynaptic mitochondria and the temporal pattern of neurotransmitter release. Philos. Trans. R. Soc. B Biol. Sci. 354, 365-372.

Bronk, P., Deák, F., Wilson, M.C., Liu, X., Südhof, T.C., and Kavalali, E.T. (2007). Differential effects of SNAP-25 deletion on $\mathrm{Ca}^{2+}$-dependent and $\mathrm{Ca}^{2+}$-independent neurotransmission. J. Neurophysiol. 98, 794-806.

Bruckner, J.J., Zhan, H., Gratz, S.J., Rao, M., Ukken, F., Zilberg, G., and O'Connor-Giles, K.M. (2017). Fife organizes synaptic vesicles and calcium channels for high-probability neurotransmitter release. J. Cell Biol. 216, 231-246.

Bruns, D., Riedel, D., Klingauf, J., and Jahn, R. (2000). Quantal Release of Serotonin. Neuron $28,205-220$.

Buchs, P.A., Stoppini, L., and Muller, D. (1993). Structural modifications associated with synaptic development in area CA1 of rat hippocampal organotypic cultures. Dev. Brain Res. $71,81-91$. 
Bullock, G.R. (1984). The current status of fixation for electron microscopy: A review. J. Microsc. 133, 1-15.

De Camilli, P., Miller, P.E., Navone, F., Theurkauf, W.E., and Vallee, R.B. (1984). Distribution of microtubule-associated protein 2 in the nervous system of the rat studied by immunofluorescence. Neuroscience 11, 819-846.

Cano, R., Ruiz, R., Shen, C., Tabares, L., and Betz, W.J. (2012). The functional landscape of a presynaptic nerve terminal. Cell Calcium 52, 321-326.

Capani, F., Martone, M.E., Deerinck, T.J., and Ellisman, M.H. (2001). Selective localization of high concentrations of F-actin in subpopulations of dendritic spines in rat central nervous system: A three-dimensional electron microscopic study. J. Comp. Neurol. 435, 156-170.

Castillo, J. del, and Katz, B. (1954). Quantal components of the end-plate potential. J. Physiol. 124, 560-573.

Castillo, P.E., Janz, R., Sudhof, T.C., Tzounopoulos, T., Malenka, R.C., and Nicoll, R.A. (1997). Rab3A is essential for mossy fibre long-term potentiation in the hippocampus. Nature 388, 590-593.

Castillo, P.E., Schoch, S., Schmitz, F., Sudhof, T.C., and Malenka, R.C. (2002). RIM1 alpha is required for presynaptic long-term potentiation. Nature 415, 327-330.

Ceccarelli, B., Hurlbut, W.P., and Mauro, A. (1973). Turnover of transmitter and synaptic vesicles at the frog neuromuscular junction. J. Cell Biol. 57, 499-524.

Ceccom, J., Halley, H., Daumas, S., and Lassalle, J.M. (2014). A specific role for hippocampal mossy fiber's zinc in rapid storage of emotional memories. Learn. Mem. 21, 287-297.

Chamberland, S., Evstratova, A., and Tóth, K. (2014). Interplay between synchronization of multivesicular release and recruitment of additional release sites support short-term facilitation at hippocampal mossy fiber to CA3 pyramidal cells synapses. J. Neurosci. 34, 11032-11047.

Chamberland, S., Timofeeva, Y., Evstratova, A., Volynski, K., and Tóth, K. (2018). Action potential counting at giant mossy fiber terminals gates information transfer in the hippocampus. Proc. Natl. Acad. Sci. U. S. A. 115, 7434-7439.

Chang, S., Trimbuch, T., and Rosenmund, C. (2018). Synaptotagmin-1 drives synchronous $\mathrm{Ca}^{2+}-$ triggered fusion by C2B-domain-mediated synaptic-vesicle-membrane attachment. Nat. Neurosci. 21, 33-42.

Chavez-Noriega, L.E., and Stevens, C.F. (1994). Increased transmitter release at excitatory synapses produced by direct activation of adenylate cyclase in rat hippocampal slices. J. Neurosci. 14, 310-317.

Chavkin, C., Bakhit, C., Weber, E., and Bloom, F.E. (1983). Relative contents and concomitant release of prodynorphin/ neoendorphin-derived peptides in rat hippocampus. Proc. Natl. Acad. Sci. U. S. A. 80, 7669-7673.

Chen, Y., Huang, C., and Hsu, K. (2001). Time-Dependent Reversal of Long-Term Potentiation by Low-Frequency Stimulation at the Hippocampal Mossy Fiber - CA3 Synapses. J. Neurosci. $21,3705-3714$.

Chen, Z., Das, B., Nakamura, Y., Digregorio, D.A., and Young, S.M. (2015). Ca ${ }^{2+}$ Channel to 
Synaptic Vesicle Distance Accounts for the Readily Releasable Pool Kinetics at a Functionally Mature Auditory Synapse. J. Neurosci. 35, 2083-2100.

Chicurel, M.E., and Harris, K.M. (1992). Three-dimensional analysis of the structure and composition of CA3 branched dendritic spines and their synaptic relationships with mossy fiber boutons in the rat hippocampus. J. Comp. Neurol. 325, 169-182.

Cho, R.W., Buhl, L.K., Volfson, D., Tran, A., Li, F., Akbergenova, Y., and Littleton, J.T. (2015). Phosphorylation of Complexin by PKA Regulates Activity-Dependent Spontaneous Neurotransmitter Release and Structural Synaptic Plasticity. Neuron 88, 749-761.

Cifuentes, F., Montoya, M., and Morales, M.A. (2008). High-frequency stimuli preferentially release large dense-core vesicles located in the proximity of nonspecialized zones of the presynaptic membrane in sympathetic ganglia. Dev. Neurobiol. 68, 446-456.

Cole, A.A., Chen, X., and Reese, T.S. (2016). A network of three types of filaments organizes synaptic vesicles for storage, mobilization, and docking. J. Neurosci. 36, 3222-3230.

Colonnier, M. (1968). Synaptic patterns on different cell types in the different laminae of the cat visual cortex. An electron microscope study. Brain Res. 9.

Coltman, B.W., Earley, E.M., Shahar, A., Dudek, F.E., and Ide, C.F. (1995). Factors influencing mossy fiber collateral sprouting in organotypic slice cultures of neonatal mouse hippocampus. J. Comp. Neurol. 362, 209-222.

Commons, K.G., and Milner, T.A. (1995). Ultrastructural Heterogeneity of EnkephalinContaining Terminals in the Rat Hippocampal Formation. J. Comp. Neurol. 358, 324-342.

Commons, K.G., and Milner, T.A. (1996). Ultrastructural relationships between leuenkephalin- and GABA-containing neurons differ within the hippocampal formation. Brain Res. 724, 1-15.

Cooper, B., Hemmerlein, M., Ammermüller, J., Imig, C., Reim, K., Lipstein, N., Kalla, S., Kawabe, H., Brose, N., Brandstätter, J.H., et al. (2012). Munc13-Independent Vesicle Priming at Mouse Photoreceptor Ribbon Synapses. J. Neurosci. 32, 8040-8052.

Cousin, M.A. (2009). Activity-Dependent Bulk Synaptic Vesicle Endocytosis-A Fast, High Capacity Membrane Retrieval Mechanism. Mol. Neurobiol. 39, 185-189.

Couteaux, R., and Pécot-Dechavassine, M. (1970). [Synaptic vesicles and pouches at the level of "active zones" of the neuromuscular junction]. Comptes rendus Hebd. des seances I'Academie des Sci. Ser. D Sci. Nat. 271, 2346-2349.

Craxton, M. (2010). A manual collection of Syt, Esyt, Rph3a, Rph3al, Doc2, and Dblc2 genes from 46 metazoan genomes - an open access resource for neuroscience and evolutionary biology. BMC Genomics 11, 1-21.

Crivellato, E., Nico, B., and Ribatti, D. (2005). Ultrastructural evidence of piecemeal degranulation in large dense-core vesicles of brain neurons. Anat. Embryol. (Berl). 210, 2534.

Cserép, C., Pósfai, B., Schwarcz, A.D., and Dénes, Á. (2018). Mitochondrial ultrastructure is coupled to synaptic performance at axonal release sites. ENeuro 5.

Dahl, R., and Staehelin, L.A. (1989). High-pressure freezing for the preservation of biological structure: theory and practice. J. Electron Microsc. Tech. 13, 165-174. 
Daniel, J.A., Malladi, C.S., Kettle, E., McCluskey, A., and Robinson, P.J. (2012). Analysis of synaptic vesicle endocytosis in synaptosomes by high-content screening. Nat. Protoc. 7, 1649-1655.

Danzer, S.C., and McNamara, J.O. (2004). Localization of Brain-Derived Neurotrophic Factor to Distinct Terminals of Mossy Fiber Axons Implies Regulation of Both Excitation and Feedforward Inhibition of CA3 Pyramidal Cells Steve. J. Neurosci. 24, 11346-11355.

Debanne, D., Guérineau, N.C., Gähwiler, B.H., and Thompson, S.M. (1996). Paired-pulse facilitation and depression at unitary synapses in rat hippocampus: Quantal fluctuation affects subsequent release. J. Physiol. 491, 163-176.

Delvendahl, I., Vyleta, N.P., von Gersdorff, H., and Hallermann, S. (2016). Fast, TemperatureSensitive and Clathrin-Independent Endocytosis at Central Synapses. Neuron 90, 492-498.

Derrick, B.E., Rodriguez, S.B., Lieberman, D.N., and Martinez, J.L. (1992). Mu Opiold Receptors Are Associated Hippocampal Mossy Fiber Long-Term antagonists. J. Pharpacology Exp. Ther. 263, 725-733.

Dieni, S., Matsumoto, T., Dekkers, M., Rauskolb, S., Ionescu, M.S., Deogracias, R., Gundelfinger, E.D., Kojima, M., Nestel, S., Frotscher, M., et al. (2012). BDNF and its propeptide are stored in presynaptic dense core vesicles in brain neurons. J. Cell Biol. 196, 775788.

Dieni, S., Nestel, S., Sibbe, M., Frotscher, M., and Hellwig, S. (2015). Distinct synaptic and neurochemical changes to the granule cell-CA3 projection in Bassoon mutant mice. Front. Synaptic Neurosci. 7, 1-14.

Dixon, D., and Atwood, H.L. (1989). Adenylate cyclase system is essential for long-term facilitation at the crayfish neuromuscular junction. J. Neurosci. 9, 4246-4252.

Dobrunz, L.E. (2002). Release probability is regulated by the size of the readily releasable vesicle pool at excitatory synapses in hippocampus. Int. J. Dev. Neurosci. 20, 225-236.

Dobrunz, L.E., and Stevens, C.F. (1997). Heterogeneity of Release Probability, Facilitation , and Depletion at Central Synapses. Neuron 18, 995-1008.

Dobrunz, L.E., and Stevens, C.F. (1999). Response of hippocampal synapses to natural stimulation patterns. Neuron 22, 157-166.

Dumas, T.C., Powers, E.C., Tarapore, P.E., and Sapolsky, R.M. (2004). Overexpression of calbindin D28K in dentate gyrus granule cells alters mossy fiber presynaptic function and impairs hippocampal-dependent memory. Hippocampus 14, 701-709.

Eccles, J.C. (1964). The Development of Ideas on the Synapse. In The Physiology of Synapses, (Berlin, Heidelberg: Springer Berlin Heidelberg), pp. 1-10.

Eccles, J.C., Katz, B., and Kuffler, S.W. (1941). Nature of the "Endplate Potential" in Curarized Muscle. J. Neurophysiol 4, 362-387.

Eggermann, E., Bucurenciu, I., Goswami, S.P., and Jonas, P. (2012). Nanodomain coupling between $\mathrm{Ca}^{2+}$ channels and sensors of exocytosis at fast mammalian synapses. Nat. Rev. Neurosci. 13, 7-21.

Eliasson, L., Ma, X., Renström, E., Barg, S., Berggren, P.O., Galvanovskis, J., Gromada, J., Jing, X., Lundquist, I., Salehi, A., et al. (2003). SUR1 regulates PKA-independent cAMP-induced 
granule priming in mouse pancreatic b-cells. J. Gen. Physiol. 121, 181-197.

Eltes, T., Kirizs, T., Nusser, Z., and Holderith, N. (2017). Target Cell Type-Dependent Differences in $\mathrm{Ca}^{2+}$ Channel Function Underlie Distinct Release Probabilities at Hippocampal Glutamatergic Terminals. Jounal Neurosci. 37, 1910-1924.

Evans, G.J.O., and Morgan, A. (2003). Regulation of the exocytotic machinery by cAMPdependent protein kinase : implications for presynaptic plasticity. Biochem. Soc. Trans. 31, 824-827.

Evstratova, A., Chamberland, S., Faundez, V., and Toth, K. (2014). Vesicles derived via AP-3 dependent recycling contribute to asynchronous release and influence information transfer. Nat. Commun. 5, 1-32.

Farina, M., van de Bospoort, R., He, E., Persoon, C.M., van Weering, J.R.T., Broeke, J.H., Verhage, M., and Toonen, R.F. (2015). CAPS-1 promotes fusion competence of stationary dense-core vesicles in presynaptic terminals of mammalian neurons. Elife 2015, 1-22.

Farrant, J.L. (1954). An electron microscopic study of ferritin. Biochim. Biophys. Acta 13, 569576.

Fasshauer, D., Otto, H., Eliason, W.K., Jahn, R., and Brünger, A.T. (1997). Structural changes are associated with soluble $\mathrm{N}$-ethylmaleimide- sensitive fusion protein attachment protein receptor complex formation. J. Biol. Chem. 272, 28036-28041.

Fasshauer, D., Antonin, W., Subramaniam, V., and Jahn, R. (2002). SNARE assembly and disassembly exhibit a pronounced hysteresis. Nat. Struct. Biol. 9, 144-151.

Fernandes, H.B., Riordan, S., Nomura, T., Remmers, C.L., Kraniotis, S., Marshall, J.J., Kukreja, L., Vassar, R., and Contractor, A. (2015). Epac2 Mediates cAMP-Dependent Potentiation of Neurotransmission in the Hippocampus. J. Neurosci. 35, 6544-6553.

Fernández-Busnadiego, R., Zuber, B., Maurer, U.E., Cyrklaff, M., Baumeister, W., and Lucic, V. (2010). Quantitative analysis of the native presynaptic cytomatrix by cryoelectron tomography. J. Cell Biol. 188, 145-156.

Fernández-Busnadiego, R., Asano, S., Oprisoreanu, A.M., Sakata, E., Doengi, M., Kochovski, Z., Zürner, M., Stein, V., Schoch, S., Baumeister, W., et al. (2013). Cryo-electron tomography reveals a critical role of RIM1 $\alpha$ in synaptic vesicle tethering. J. Cell Biol. 201, 725-740.

Fernández-Chacón, R., Königstorfer, A., Gerber, S.H., García, J., Matos, M.F., Stevens, C.F., Brose, N., Rizo, J., Rosenmund, C., and Südhof, T.C. (2001). Synaptotagmin I functions as a calcium regulator of release probability. Nature 410, 41-49.

Fiala, J.C., Feinberg, M., Popov, V., and Harris, K.M. (1998). Synaptogenesis Via Dendritic Filopodia in Developing Hippocampal Area CA1. J. Neurosci. 18, 8900-8911.

Finck, H. (1960). Epoxy Resins in Electron Microscopy. J. Biophys. Biochem. Cytol. 7, 27-30.

Frotscher, M., and Gähwiler, B.H. (1988). Synaptic organization of intracellularly stained CA3 pyramidal neurons in slice cultures of rat hippocampus. Neuroscience 24, 541-551.

Frotscher, M., Jonas, P., and Sloviter, R.S. (2006). Synapses formed by normal and abnormal hippocampal mossy fibers. Cell Tissue Res. 326, 361-367.

Frotscher, M., Studer, D., Graber, W., Chai, X., Nestel, S., and Zhao, S. (2014). Fine structure of synapses on dendritic spines. Front. Neuroanat. 8, 94. 
Fujita, Y., Shirataki, H., Sakisaka, T., Asakura, T., Ohya, T., Kotani, H., Yokoyama, S., Nishioka, H., Matsuura, Y., Mizoguchi, A., et al. (1998). Tomosyn: A syntaxin-1-binding protein that forms a novel complex in the neurotransmitter release process. Neuron 20, 905-915.

Fykse, E.M., Li, C., and Sudhof, T.C. (1995). Phosphorylation of rabphilin-3A by $\mathrm{Ca}^{2+} /$ calmodulin- and cAMP-dependent protein kinases in vitro. J. Neurosci. 15, 2385-2395.

Gähwiler, B.H. (1984). Development of the hippocampus in vitro: Cell types, synapses and receptors. Neuroscience 11, 751-760.

Galimberti, I., Gogolla, N., Alberi, S., Santos, A.F., Muller, D., and Caroni, P. (2006). Long-Term Rearrangements of Hippocampal Mossy Fiber Terminal Connectivity in the Adult Regulated by Experience. Neuron 50, 749-763.

Geiger, J.R.P., and Jonas, P. (2000). Dynamic control of presynaptic $\mathrm{Ca}^{2+}$ inflow by fastinactivating $\mathrm{K}^{+}$channels in hippocampal mossy fiber boutons. Neuron 28, 927-939.

Gentet, L.J., Stuart, G.J., and Clements, J.D. (2000). Direct measurement of specific membrane capacitance in neurons. Biophys. J. 79, 314-320.

Geppert, M., Goda, Y., Stevens, C.F., and Südhof, T.C. (1997). The small GTP-binding protein Rab3A regulates a late step in synaptic vesicle fusion. Nature $387,810-814$.

Gibson, H.E., Reim, K., Brose, N., Morton, A.J., and Jones, S. (2005). A similar impairment in CA3 mossy fibre LTP in the R6/2 mouse model of Huntington's disease and in the complexin II knockout mouse. Eur. J. Neurosci. 22, 1701-1712.

Giddings, T.H. (2003). Freeze-substitution protocols for improved visualization of membranes in high-pressure frozen samples. J. Microsc. 212, 53-61.

Gondré-Lewis, M.C., Park, J.J., and Loh, Y.P. (2012). Cellular Mechanisms for the Biogenesis and Transport of Synaptic and Dense-Core Vesicles. In International Review of Cell and Molecular Biology, K.W. Jeon, ed. (Boston: Academic Press), pp. 27-115.

Gonzales, R.B., Galvan, C.J.D.L., Rangel, Y.M., and Claiborne, B.J. (2001). Distribution of thorny excrescences on CA3 pyramidal neurons in the rat hippocampus. J. Comp. Neurol. 430, 357368.

Govind, C.K., Pearce, J., Wojtowicz, J.M., and Atwood, H.L. (1994). "Strong " and "Weak " Synaptic Differentiation in the Crayfish Opener Muscle: Structural Correlates. Nature Rev. Neurosci. 58, 45-58.

Grabs, D., Bergmann, M., Schuster, T., Fox, P.A., Brich, M., and Gratz, M. (1994). Differential Expression of Synaptophysin and Synaptoporin During Pre- and Postnatal Development of the Rat Hippocampal Network. Eur. J. Neurosci. 6, 1765-1771.

Gray, E.G. (1959). Axo-somatic and axo-dendritic synapses of the cerebral cortex: an electron microscope study. J. Anat. 93, 420-433.

Gustafsson, B., Ma, R., and Hanse, E. (2019). The Small and Dynamic Pre-primed Pool at the Release Site; A Useful Concept to Understand Release Probability and Short-Term Synaptic Plasticity? Front. Synaptic Neurosci. 11, 1-14.

Hallermann, S., and Silver, R.A. (2013). Sustaining rapid vesicular release at active zones: Potential roles for vesicle tethering. Trends Neurosci. 36, 185-194.

Hallermann, S., Pawlu, C., Jonas, P., and Heckmann, M. (2003). A large pool of releasable 
vesicles in a cortical glutamatergic synapse. Proc. Natl. Acad. Sci. U. S. A. 100, 8975-8980.

Hallermann, S., Fejtova, A., Schmidt, H., Weyhersmüller, A., Silver, R.A., Gundelfinger, E.D., and Eilers, J. (2010). Bassoon Speeds Vesicle Reloading at a Central Excitatory Synapse. Neuron 68, 710-723.

Hammarlund, M., Palfreyman, M.T., Watanabe, S., Olsen, S., and Jorgensen, E.M. (2007). Open syntaxin docks synaptic vesicles. PLoS Biol. 5, 1695-1711.

Han, Y., Kaeser, P.S., Südhof, T.C., and Schneggenburger, R. (2011). RIM determines Ca ${ }^{2+}$ channel density and vesicle docking at the presynaptic active zone. Neuron 69, 304-316.

Hanse, E., and Gustafsson, B. (2001). Vesicle release probability and pre-primed pool at glutamatergic synapses in area CA1 of the rat neonatal hippocampus. J. Physiol. 531, 481493.

Hanson, P.I., Roth, R., Morisaki, H., Jahn, R., and Heuser, J.E. (1997). Structure and conformational changes in NSF and its membrane receptor complexes visualized by quickfreeze/deep-etch electron microscopy. Cell 90, 523-535.

Harris, K.M., and Stevens, J.K. (1989). Dendritic spines of CA1 pyramidal cells in the rat hippocampus: serial electron microscopy with reference to their biophysical characteristics. J. Neurosci. 9, 2982-2997.

Harris, K.M., and Sultan, P. (1995). Variation in the number, location and size of synaptic vesicles provides an anatomical basis for the nonuniform probability of release at hippocampal CA1 synapses. Neuropharmacology 34, 1387-1395.

Harris, K.M., and Weinberg, R.J. (2012). Ultrastructure of synapses in the mammalian brain. Cold Spring Harb. Perspect. Biol. 4, 7.

Harris, K., Jensen, F., and Tsao, B. (1992). Three-dimensional structure of dendritic spines and synapses in rat hippocampus (CA1) at postnatal day 15 and adult ages: implications for the maturation of synaptic physiology and long-term potentiation. J. Neurosci. 12, 2685-2705.

Harrison, R.G. (1910). The outgrowth of the nerve fiber as a mode of protoplasmic movement. J. Exp. Zool. 9, 787-846.

Hatsuzawa, K., Lang, T., Fasshauer, D., Bruns, D., and Jahn, R. (2003). The R-SNARE motif of tomosyn forms SNARE core complexes with syntaxin 1 and SNAP-25 and down-regulates exocytosis. J. Biol. Chem. 278, 31159-31166.

Hayat, M.A. (1981). Principles and techniques of electron microscopy. Biological applications. (Cambridge University Press).

He, L., Xue, L., Xu, J., McNeil, B.D., Bai, L., Melicoff, E., Adachi, R., and Wu, L.G. (2009). Compound vesicle fusion increases quantal size and potentiates synaptic transmission. Nature 459, 93-97.

Helassa, N., Dürst, C.D., Coates, C., Kerruth, S., Arif, U., Schulze, C., Wiegert, J.S., Geeves, M., Oertner, T.G., and Török, K. (2018). Ultrafast glutamate sensors resolve high-frequency release at Schaffer collateral synapses. Proc. Natl. Acad. Sci. 115, 5594-5599.

Henze, D.., Urban, N.., and Barrionuevo, G. (2000). The multifarious hippocampal mossy fiber pathway: a review. Neuroscience 98, 407-427.

Henze, D.A., Card, J.P., Barrionuevo, G., and Ben-Ari, Y. (1997). Large amplitude miniature 
excitatory postsynaptic currents in hippocampal CA3 pyramidal neurons are of mossy fiber origin. J.Neurophysiol. 77, 1075-1086.

Henze, D.A., Wittner, L., and Buzsáki, G. (2002a). Single granule cells reliably discharge targets in the hippocampal CA3 network in vivo. Nat. Neurosci. 5, 790-795.

Henze, D.A., McMahon, D.B.T., Harris, K.M., and Barrionuevo, G. (2002b). Giant miniature EPSCs at the hippocampal mossy fiber to CA3 pyramidal cell synapse are monoquantal. J. Neurophysiol. 87, 15-29.

Heuser, J.E., and Reese, T.S. (1973). Evidence for recycling of synaptic vesicle membrane during transmitter release at the frog neuromuscular junction. J. Cell Biol. 57, 315-344.

High, B., Cole, A.A., Chen, X., and Reese, T.S. (2015). Electron microscopic tomography reveals discrete transcleft elements at excitatory and inhibitory synapses. Front. Synaptic Neurosci. 7, 1-9.

Ho, M.T.-W., Pelkey, K.A., Topolnik, L., Petralia, R.S., Takamiya, K., Xia, J., Huganir, R.L., Lacaille, J.-C., and McBain, C.J. (2007). Developmental Expression of Ca ${ }^{2+}$-Permeable AMPA Receptors Underlies Depolarization-Induced Long-Term Depression at Mossy Fiber CA3 Pyramid Synapses. J. Neurosci. 27, 11651-11662.

Holderith, N., Lorincz, A., Katona, G., Rózsa, B., Kulik, A., Watanabe, M., and Nusser, Z. (2012). Release probability of hippocampal glutamatergic terminals scales with the size of the active zone. Nat. Neurosci. 15, 988-997.

Hopwood, D. (1969). Fixatives and fixation: a review. Histochem. J. 1, 323-360.

Huang, C., Chen, Y., Liang, Y., and Hsu, K. (2002). Role for cAMP and protein phosphatase in the presynaptic expression of mouse hippocampal mossy fibre depotentiation. J. Physiol. 543, 767-778.

Huang, Y.-Y., Li, X.-C., and Kandel, E.R. (1994a). cAMP contributes to mossy fiber LTP by initiating both a covalently mediated early phase and macromolecular synthesis-dependent late phase. Cell 79, 69-79.

Huang, Y., Li, X.-C., Kandel, E.R., Alberini, C.M., Ghirardi, M., Metz, R., Kandel, E.R., Alkon, D.L., Rasmussen, H., Amaral, D.G., et al. (1994b). cAMP contributes to mossy fiber LTP by initiating both a covalently mediated early phase and macromolecular synthesis-dependent late phase. Cell 79, 69-79.

Huang, Y.Z., Pan, E., Xiong, Z.Q., and McNamara, J.O. (2008). Zinc-Mediated Transactivation of TrkB Potentiates the Hippocampal Mossy Fiber-CA3 Pyramid Synapse. Neuron 57, 546558.

Hui, E., Bai, J., Wang, P., Sugimori, M., Llinas, R.R., and Chapman, E.R. (2005). Three distinct kinetic groupings of the synaptotagmin family: Candidate sensors for rapid and delayed exocytosis. Proc. Natl. Acad. Sci. U. S. A. 102, 5210-5214.

Humpel, C. (2016). Organotypic brain slice cultures: a review. Neuroscience 305, 86-98.

Hussain, R.J., and Carpenter, D.O. (2001). Development of synaptic responses and plasticity at the SC-CA1 and MF-CA3 synapses in rat hippocampus. Cell. Mol. Neurobiol. 21, 357-368.

Ikeda, S.R., Lovinger, D.M., McCool, B.A., and Lewis, D.L. (1995). Heterologous expression of metabotropic glutamate receptors in adult rat sympathetic neurons: subtype-specific 
coupling to ion channels. Neuron $14,1029-1038$.

Imig, C., and Cooper, B.H. (2017). 3D Analysis of Synaptic Ultrastructure in Organotypic Hippocampal Slice Culture by High-Pressure Freezing and Electron Tomography. In Synapse Development, J.M. Walker, and A. Poulopoulos, eds. (New York: Springer Nature), pp. 215231.

Imig, C., Min, S.-W., Krinner, S., Arancillo, M., Rosenmund, C., Südhof, T.C., Rhee, J., Brose, N., and Cooper, B.H. (2014). The morphological and molecular nature of synaptic vesicle priming at presynaptic active zones. Neuron 84, 416-431.

Ishikawa, T., Nakamura, Y., Saitoh, N., Li, W. Bin, Iwasaki, S., and Takahashi, T. (2003). Distinct Roles of Kv1 and Kv3 Potassium Channels at the Calyx of Held Presynaptic Terminal. J. Neurosci. 23, 10445-10453.

Ivannikov, M. V., Sugimori, M., and Llinás, R.R. (2013). Synaptic vesicle exocytosis in hippocampal synaptosomes correlates directly with total mitochondrial volume. J. Mol. Neurosci. 49, 223-230.

Jackman, S.L., and Regehr, W.G. (2017). The Mechanisms and Functions of Synaptic Facilitation. Neuron 94, 447-464.

Jackman, S.L., Turecek, J., Belinsky, J.E., and Regehr, W.G. (2016). The calcium sensor synaptotagmin 7 is required for synaptic facilitation. Nature 529, 88-91.

Jahn, R., Lang, T., and Südhof, T.C. (2003). Membrane fusion. Cell 112, 519-533.

Jarrard, L.E. (1993). On the role of the hippocampus in learning and memory in the rat. Behav. Neural Biol. 60, 9-26.

Jockusch, W.J., Speidel, D., Sigler, A., Sørensen, J.B., Varoqueaux, F., Rhee, J.S., and Brose, N. (2007). CAPS-1 and CAPS-2 Are Essential Synaptic Vesicle Priming Proteins. Cell 131, 796-808.

Jonas, P., Major, G., and Sakmann, B. (1993). Quantal components of unitary EPSCs at the mossy fibre synapse on CA3 pyramidal cells of rat hippocampus. J. Physiol. 472, 615-663.

Kaeser-Woo, Y.J., Younts, T.J., Yang, X., Zhou, P., Wu, D., Castillo, P.E., and Südhof, T.C. (2013). Synaptotagmin-12 Phosphorylation by cAMP-Dependent Protein Kinase Is Essential for Hippocampal Mossy Fiber LTP. J. Neurosci. 33, 9769-9780.

Kaeser, P.S., and Regehr, W.G. (2017). The readily releasable pool of synaptic vesicles. Curr. Opin. Neurobiol. 43, 63-70.

Kamiya, H., and Ozawa, S. (1999). Dual mechanism for presynaptic modulation by axonal metabotropic glutamate receptor at the mouse mossy fibre-CA3 synapse. J. Physiol. 518 ( Pt 2, 497-506.

Kamiya, H., Shinozaki, H., and Yamamoto, C. (1996). Activation of metabotropic glutamate receptor type 2/3 suppresses transmission at rat hippocampal mossy fibre synapses. J. Physiol. 1, 447-455.

Kang, G., Joseph, J.W., Chepurny, O.G., Monaco, M., Wheeler, M.B., Bos, J.L., Schwede, F., Genieser, H.G., and Holz, G.G. (2003). Epac-selective cAMP analog 8-pCPT-2'-O-Me-cAMP as a stimulus for $\mathrm{Ca}^{2+}$-induced $\mathrm{Ca}^{2+}$ release and exocytosis in pancreatic $\beta$-cells. J. Biol. Chem. 278, 8279-8285.

Kanno, H., Speedy, R.J., and Angell, C.A. (1975). Supercooling of Water to -92C Under 
Pressure. Science 189, 880-881.

Kawamura, Y., Manita, S., Nakamura, T., Inoue, M., Kudo, Y., and Miyakawa, H. (2004). Glutamate release increases during mossy-CA3 LTP but not during Schaffer-CA1 LTP. Eur. J. Neurosci. 19, 1591-1600.

Keller, D., Babai, N., Kochubey, O., Han, Y., Markram, H., Schürmann, F., and Schneggenburger, R. (2015). An Exclusion Zone for $\mathrm{Ca}^{2+}$ Channels around Docked Vesicles Explains Release Control by Multiple Channels at a CNS Synapse. PLoS Comput. Biol. 11,1-21.

Kohara, K., Pignatelli, M., Rivest, A.J., Jung, H.-Y., Kitamura, T., Suh, J., Frank, D., Kajikawa, K., Mise, N., Obata, Y., et al. (2014). Cell type-specific genetic and optogenetic tools reveal novel hippocampal CA2 circuits. Nat. Neurosci. 17, 269-279.

Korogod, N., Petersen, C.C.H., and Knott, G.W. (2015). Ultrastructural analysis of adult mouse neocortex comparing aldehyde perfusion with cryo fixation. Elife 4, 1-17.

Koster, A.J., Grimm, R., Typke, D., Hegerl, R., Stoschek, A., Walz, J., and Baumeister, W. (1997). Perspectives of molecular and cellular electron tomography. J. Struct. Biol. 120, 276-308.

Kremer, J.R., Mastronarde, D.N., and McIntosh, J.R. (1996). Computer Visualization of ThreeDimensional Image Data Using IMOD. J. Struct. Biol. 116, 71-76.

Kusick, G.F., Chin, M., Lippmann, K., Adula, K.P., Davis, M.W., Jorgensen, E.M., and Watanabe, S. (2020). Synaptic vesicles undock and then transiently dock after an action potential. BioRxiv.

Kwon, S.K., Sando, R., Lewis, T.L., Hirabayashi, Y., Maximov, A., and Polleux, F. (2016). LKB1 Regulates Mitochondria-Dependent Presynaptic Calcium Clearance and Neurotransmitter Release Properties at Excitatory Synapses along Cortical Axons. PLoS Biol. 14, 1-27.

Laatsch, R.H., and Cowan, W.M. (1966). Electron microscopic studies of the dentate gyrus of the rat. I. Normal structure with special reference to synaptic organization. J. Comp. Neurol. 128, 359-395.

Landis, D.M.D., Hall, A.K., Weinstein, L.A., and Reese, T.S. (1988). The organization of cytoplasm at the presynaptic active zone of a central nervous system synapse. Neuron 1,201209.

Lanore, F., Blanchet, C., Fejtova, A., Pinheiro, P.S., Richter, K., Balschun, D., Gundelfinger, E.D., and Mulle, C. (2010). Impaired development of hippocampal mossy fibre synapses in mouse mutants for the presynaptic scaffold protein Bassoon. J Physiol 588, 2133-2145.

LaVail, J.H., and Wolf, M.K. (1973). Postnatal Development of the Mouse Dentate Gyrus in Organotypic Cultures of the Hippocampal Formation. Am. J. Anat. 137, 447-466.

Lavoie, N., Jeyaraju, D. V, Peralta, M.R., Seress, L., Pellegrini, L., and Toth, K. (2011). Vesicular Zinc Regulates the $\mathrm{Ca}^{2+}$ Sensitivity of a Subpopulation of Presynaptic Vesicles at Hippocampal Mossy Fiber Terminals. J. Neurosci. 31, 18251-18265.

Lawrence, J.J., Grinspan, Z.M., and McBain, C.J. (2004). Quantal transmission at mossy fibre targets in the CA3 region of the rat hippocampus. J. Physiol. 554, 175-193.

Lee, S.H., Kim, K.R., Ryu, S.Y., Son, S., Hong, H.S., Mook-Jung, I., Lee, S.H., and Ho, W.K. (2012). Impaired short-term plasticity in mossy fiber synapses caused by mitochondrial dysfunction of dentate granule cells is the earliest synaptic deficit in a mouse model of Alzheimer's 
disease. J. Neurosci. 32, 5953-5963.

Li, Y., Calfa, G., Inoue, T., Amaral, M.D., and Pozzo-Miller, L. (2010). Activity-dependent release of endogenous BDNF from mossy fibers evokes a TRPC3 current and $\mathrm{Ca}^{2+}$ elevations in CA3 pyramidal neurons. J. Neurophysiol. 103, 2846-2856.

Lin, R.C., and Scheller, R.H. (1997). Structural organization of the synaptic exocytosis core complex. Neuron 19, 1087-1094.

Link, E., Edelmann, L., Chou, J.H., Binz, T., Yamasaki, S., Eisel, U., Baumert, M., Südhof, T.C., Niemann, H., and Jahn, R. (1992). Tetanus toxin action: Inhibition of neurotransmitter release linked to synaptobrevin proteolysis. Biochem. Biophys. Res. Commun. 189, 1017-1023.

Loewen, J.J., Peters, R.I., and Terrian, D.M. (1992). Adenosine modulation of dynorphin B release by hippocampal synaptosomes. Brain Res. 577, 318-320.

Lonart, G., and Sudhof, T.C. (1998). Region-Specific Phosphorylation of Rabphilin in Mossy Fiber Nerve Terminals of the Hippocampus. J Neurosci 18, 634-640.

López-García, J.C., Arancio, O., Kandel, E.R., and Baranes, D. (1996). A presynaptic locus for long-term potentiation of elementary synaptic transmission at mossy fiber synapses in culture. Proc. Natl. Acad. Sci. U. S. A. 93, 4712-4717.

Lučić, V., Förster, F., and Baumeister, W. (2005). Structural Studies by Electron Tomography: From Cells to Molecules. Annu. Rev. Biochem. 74, 833-865.

Luiten, P.G.M., Buwalda, B., Traber, J., and Nyakas, C. (1994). Induction of enhanced postnatal expression of immunoreactive calbindin-D28k in rat forebrain by the calcium antagonist nimodipine. Dev. Brain Res. 79, 10-18.

Ma, C., Li, W., Xu, Y., and Rizo, J. (2011). Munc13 mediates the transition from the closed syntaxin-Munc18 complex to the SNARE complex. Nat. Struct. Mol. Biol. 18, 542-549.

Maas, C., Torres, V.I., Altrock, W.D., Leal-Ortiz, S., Wagh, D., Terry-Lorenzo, R.T., Fejtova, A., Gundelfinger, E.D., Ziv, N.E., and Garner, C.C. (2012). Formation of Golgi-derived active zone precursor vesicles. J. Neurosci. 32, 11095-11108.

Maccaferri, G., Toth, K., and McBain, C.J. (1998). Target-Specific Expression of Presynaptic Mossy Fiber Plasticity. Science 279, 1368-1371.

Madisen, L., Mao, T., Koch, H., Zhuo, J., Berenyi, A., Fujisawa, S., Hsu, Y.A., Garcia, A.J.I., Gu, X., Zanella, S., et al. (2012). A toolbox of Cre-dependent optogenetic transgenic mice for lightinduced activation and silencing Linda. Nat. Neurosci. 15, 793-802.

Mahamid, J., Schampers, R., Persoon, H., Hyman, A.A., Baumeister, W., and Plitzko, J.M. (2015). A focused ion beam milling and lift-out approach for site-specific preparation of frozen-hydrated lamellas from multicellular organisms. J. Struct. Biol. 192, 262-269.

Malinin, V.S., Frederik, P., and Lentz, B.R. (2002). Osmotic and curvature stress affect PEGinduced fusion of lipid vesicles but not mixing of their lipids. Biophys. J. 82, 2090-2100.

Man, K.N.M., Imig, C., Walter, A.M., Pinheiro, P.S., Stevens, D.R., Rettig, J., Sørensen, J.B., Cooper, B.H., Brose, N., and Wojcik, S.M. (2015). Identification of a Munc13-sensitive step in chromaffin cell large dense-core vesicle exocytosis. Elife 4, e10635.

Marchal, C., and Mulle, C. (2004). Postnatal maturation of mossy fibre excitatory transmission in mouse CA3 pyramidal cells: A potential role for kainate receptors. J. Physiol. 561, 27-37. 
Mastronarde, D.N. (2005). Automated electron microscope tomography using robust prediction of specimen movements. J. Struct. Biol. 152, 36-51.

Maus, L., Lee, C., Altas, B., Sertel, S.M., Weyand, K., Rizzoli, S.O., Rhee, J., Brose, N., Imig, C., and Cooper, B.H. (2020). Ultrastructural Correlates of Presynaptic Functional Heterogeneity in Hippocampal Synapses. Cell Rep. 30, 3632-3643.e8.

Maximov, A., Shin, O.H., Liu, X., and Südhof, T.C. (2007). Synaptotagmin-12, a synaptic vesicle phosphoprotein that modulates spontaneous neurotransmitter release. J. Cell Biol. 176, 113124.

Mccluskey, A., Daniel, J.A., Hadzic, G., Chau, N., Clayton, E.L., Mariana, A., Whiting, A., Gorgani, N.N., Lloyd, J., Quan, A., et al. (2013). Building a better dynasore: The dyngo compounds potently inhibit dynamin and endocytosis. Traffic 14, 1272-1289.

McMahon, H.T., and Boucrot, E. (2011). Molecular mechanism and physiological functions of clathrin-mediated endocytosis. Nat. Rev. Mol. Cell Biol. 12, 517-533.

McQuiston, a R., and Colmers, W.F. (1996). Neuropeptide Y2 receptors inhibit the frequency of spontaneous but not miniature EPSCS in CA3 pyramidal cells of rat hippocampus. J. Neurophysiol. 76, 3159-3168.

Mennerick, S., and Zorumski, C.F. (1995). Paired-pulse modulation of fast excitatory synaptic currents in microcultures of rat hippocampal neurons. J. Physiol. 488, 85-101.

Midorikawa, M., and Sakaba, T. (2017). Kinetics of Releasable Synaptic Vesicles and Their Plastic Changes at Hippocampal Mossy Fiber Report Kinetics of Releasable Synaptic Vesicles and Their Plastic Changes at Hippocampal Mossy Fiber Synapses. Neuron 96, 1033-1040.e3.

Miki, T., Kaufmann, W.A., Malagon, G., Gomez, L., Tabuchi, K., Watanabe, M., Shigemoto, R., and Marty, A. (2017). Numbers of presynaptic $\mathrm{Ca}^{2+}$ channel clusters match those of functionally defined vesicular docking sites in single central synapses. Proc. Natl. Acad. Sci. U. S. A. 114, E5246-E5255.

Miki, T., Midorikawa, M., and Sakaba, T. (2020). Direct imaging of rapid tethering of synaptic vesicles accompanying exocytosis at a fast central synapse. Proc. Natl. Acad. Sci. 202000265.

Millar, A.G., Bradacs, H., Charlton, M.P., and Atwood, H.L. (2002). Inverse relationship between release probability and readily releasable vesicles in depressing and facilitating synapses. J. Neurosci. 22, 9661-9667.

Millet, L.J., and Gillette, M.U. (2012). Over a century of neuron culture: from the hanging drop to microfluidic devices. Yale J. Biol. Med. 85, 501-521.

Möbius, W., Cooper, B., Kaufmann, W.A., Imig, C., Ruhwedel, T., Snaidero, N., Saab, A.S., and Varoqueaux, F. (2010). Electron microscopy of the mouse central nervous system. Methods Cell Biol. 96, 475-512.

Mongiat, L.A., and Schinder, A.F. (2011). Adult neurogenesis and the plasticity of the dentate gyrus network. Eur. J. Neurosci. 33, 1055-1061.

Moor, H. (1987). Theory and Practice of High Pressure Freezing. In Cryotechniques in Biological Electron Microscopy, R.A. Steinbrecht, and K. Zierold, eds. (Berlin Heidelberg: Springer-Verlag), pp. 175-191.

Moor, H., Bellin, G., Sandri, C., and Akert, K. (1980). The influence of high pressure freezing 
on mammalian nerve tissue. Cell Tissue Res. 209, 201-216.

Mori-Kawakami, F., Kobayashi, K., and Takahashi, T. (2003). Developmental decrease in synaptic facilitation at the mouse hippocampal mossy fibre synapse. J. Physiol. 553, 37-48.

Mukherjee, K., Yang, X., Gerber, S.H., Kwon, H.B., Ho, A., Castillo, P.E., Liu, X., and Südhof, T.C. (2010). Piccolo and bassoon maintain synaptic vesicle clustering without directly participating in vesicle exocytosis. Proc. Natl. Acad. Sci. U. S. A. 107, 6504-6509.

Muller, D., Buchs, P.A., and Stoppini, L. (1993). Time course of synaptic development in hippocampal organotypic cultures. Dev. Brain Res. 71, 93-100.

Müller, A., Kukley, M., Stausberg, P., Beck, H., Müller, W., and Dietrich, D. (2005). Endogenous $\mathrm{Ca}^{2+}$ buffer concentration and $\mathrm{Ca}^{2+}$ microdomains in hippocampal neurons. J. Neurosci. 25, 558-565.

Münster-Wandowski, A., Gómez-Lira, G., and Gutiérrez, R. (2013). Mixed neurotransmission in the hippocampal mossy fibers. Front. Cell. Neurosci. 7, 1-19.

Murk, J.L.A.N., Posthuma, G., Koster, A.J., Geuze, H.J., Verkleij, A.J., Kleijmeer, M.J., and Humbel, B.M. (2003). Influence of aldehyde fixation on the morphology of endosomes and lysosomes: quantitative analysis and electron tomography. J. Microsc. 212, 81-90.

Nägerl, U.V., Novo, D., Mody, I., and Vergara, J.L. (2000). Binding kinetics of calbindin-D(28k) detemined by flash photolysis of caged $\mathrm{Ca}^{2+}$. Biophys. J. 79, 3009-3018.

Nakamura, M., Sekino, Y., and Manabe, T. (2007). GABAergic interneurons facilitate mossy fiber excitability in the developing hippocampus. J. Neurosci. 27, 1365-1373.

Nakamura, Y., Reva, M., and Digregorio, D.A. (2018). Variations in $\mathrm{Ca}^{2+}$ influx can alter chelator-based estimates of $\mathrm{Ca}^{2+}$ channel-synaptic vesicle coupling distance. J. Neurosci. 38, 3971-3987.

Narahashi, T., Moore, J.W., and Scott, W.R. (1964). Tetrodotoxin Blockage of Sodium Conductance Increase in Lobster Giant Axons. J. Gen. Physiol. 47, 965-974.

Neher, E. (2015). Merits and Limitations of Vesicle Pool Models in View of Heterogeneous Populations of Synaptic Vesicles. Neuron 87, 1131-1142.

Neher, E., and Brose, N. (2018). Dynamically Primed Synaptic Vesicle States: Key to Understand Synaptic Short-Term Plasticity. Neuron 100, 1283-1291.

Neher, E., and Marty, A. (1982). Discrete changes of cell membrane capacitance observed under conditions of enhanced secretion in bovine adrenal chromaffin cells. Proc. Natl. Acad. Sci. U. S. A. 79, 6712-6716.

Neves, G., Cooke, S.F., and Bliss, T.V.P. (2008). Synaptic plasticity, memory and the hippocampus : a neural network approach to causality. Nat. Rev. Neurosci. 9, 65-75.

Nguyen, P. V., Marin, L., and Atwood, H.L. (1997). Synaptic physiology and mitochondrial function in crayfish tonic and phasic motor neurons. J. Neurophysiol. 78, 281-294.

Nicoll, R.A., and Schmitz, D. (2005). Synaptic plasticity at hippocampal mossy fibre synapses. Nat. Rev. Neurosci. 6, 863-876.

Oertner, T.G., Sabatini, B.L., Nimchinsky, E.A., and Svoboda, K. (2002). Facilitation at single synapses probed with optical quantal analysis. Nat. Neurosci. 5, 657-664. 
Okabe, S. (2007). Molecular anatomy of the postsynaptic density. Mol. Cell. Neurosci. 34, 503-518.

Palay, S.L. (1956). Synapses in the central nervous system. J. Biophys. Biochem. Cytol. 2, 193202.

Palay, S.L., and Palade, G.E. (1955). The fine structure of neurons. J. Biophys. Biochem. Cytol. 1, 69-88.

Pang, Z.P., and Südhof, T.C. (2010). Cell biology of Ca ${ }^{2+}$-triggered exocytosis. Curr. Opin. Cell Biol. 22, 496-505.

Perkins, G.A., Tjong, J., Brown, J.M., Poquiz, P.H., Scott, R.T., Kolson, D.R., Ellisman, M.H., and Spirou, G.A. (2010). The Micro-Architecture of Mitochondria at Active Zones: Electron Tomography Reveals Novel Anchoring Scaffolds and Cristae Structured for High-Rate Metabolism. J. Neurosci. 30, 1015-1026.

Pernía-Andrade, A.J., Goswami, S.P., Stickler, Y., Fröbe, U., Schlögl, A., and Jonas, P. (2012). A Deconvolution-Based Method with High Sensitivity and Temporal Resolution for Detection of Spontaneous Synaptic Currents In Vitro and In Vivo. Biophys. J. 103, 1429-1439.

Phillips, G.R., Huang, J.K., Wang, Y., Tanaka, H., Shapiro, L., Zhang, W., Shan, W.S., Arndt, K., Frank, M., Gordon, R.E., et al. (2001). The presynaptic particle web: Ultrastructure, composition, dissolution, and reconstitution. Neuron 32, 63-77.

Purves, D., Augustine, G.J., Fitzpatrick, D., Hall, W.C., LaMantia, A.-S., McNamara, J.O., and Williams, S.M. (2004). Neuroscience (Sunderland: Sinauer Associates Inc.).

Rangaraju, V., Calloway, N., and Ryan, T.A. (2014). Activity-driven local ATP synthesis is required for synaptic function. Cell 156, 825-835.

Rebola, N., Reva, M., Kirizs, T., Szoboszlay, M., Lőrincz, A., Moneron, G., Nusser, Z., and DiGregorio, D.A. (2019). Distinct Nanoscale Calcium Channel and Synaptic Vesicle Topographies Contribute to the Diversity of Synaptic Function. Neuron 1-18.

Richmond, J.E., Davis, W.S., and Jorgensen, E.M. (1999). UNC-13 is required for synaptic vesicle fusion in C. elegans. Nat. Neurosci. 2, 959-964.

Rizzoli, S.O., and Betz, W.J. (2004). The structural organization of the readily releasable pool of synaptic vesicles. Science 303, 2037-2039.

Rizzoli, S.O., and Betz, W.J. (2005). Synaptic vesicle pools. Nat. Rev. Neurosci. 6, 57-69.

De Robertis, E.D.P., and Bennett, H.S. (1955). Some features of the submicroscopic morphology of synapses in frog and earthworm. J. Biophys. Biochem. Cytol. 1, 47-58.

Robichaux, W.G., and Cheng, X. (2018). Intracellular cAMP Sensor EPAC: Physiology, Pathophysiology, and Therapeutics Development. Physiol. Rev. 98, 919-1053.

Rollenhagen, A., Sätzler, K., Rodríguez, E.P., Jonas, P., Frotscher, M., and Lübke, J.H.R. (2007). Structural determinants of transmission at large hippocampal mossy fiber synapses. J. Neurosci. 27, 10434-10444.

De Rooij, J., Zwartkruis, F.J.T., Verheijen, M.H.G., Cool, R.H., Nijman, S.M.B., Wittinghofer, A., and Bos, J.L. (1998). Epac is a Rap1 guanine-nucleotide-exchange factor directly activated by cyclic AMP. Nature 396, 474-477. 
Rose, T., Schoenenberger, P., Jezek, K., and Oertner, T.G. (2013). Developmental Refinement of Vesicle Cycling at Schaffer Collateral Synapses. Neuron 77, 1109-1121.

Rosenmund, C., and Stevens, C.F. (1996). Definition of the Readily Releasable Pool of Vesicles at Hippocampal Synapses. Neuron 16, 1197-1207.

Rosenmund, C., and Stevens, C.F. (1997). The rate of aldehyde fixation of the exocytotic machinery in cultured hippocampal synapses. J. Neurosci. Methods 76, 1-5.

De Rosier, D.J., and Klug, A. (1968). Reconstruction of three dimensional fiber structures from orthogonal projections. Nature 217, 130-134.

Rostaing, P., Real, E., Siksou, L., Lechaire, J.P., Boudier, T., Boeckers, T.M., Gertler, F., Gundelfinger, E.D., Triller, A., and Marty, S. (2006). Analysis of synaptic ultrastructure without fixative using high-pressure freezing and tomography. Eur. J. Neurosci. 24, 3463-3474.

Ruska, E. (1933). Zur Fokussierbarkeit von Kathodenstrahlbündeln großer Ausgangsquerschnitte. Zeitschrift Für Phys. 83, 684-697.

Sadakata, T., Kakegawa, W., Shinoda, Y., Hosono, M., Katoh-Semba, R., Sekine, Y., Sato, Y., Tanaka, M., Iwasato, T., Itohara, S., et al. (2013). CAPS1 deficiency perturbs dense-core vesicle trafficking and golgi structure and reduces presynaptic release probability in the mouse brain. J. Neurosci. 33, 17326-17334.

Sai, K., Wang, S., Kaito, A., Fujiwara, T., Maruo, T., Itoh, Y., Miyata, M., Sakakibara, S., Miyazaki, N., Murata, K., et al. (2017). Multiple roles of afadin in the ultrastructural morphogenesis of mouse hippocampal mossy fiber synapses. J. Comp. Neurol. 525, 2719-2734.

Salin, P. a, Weisskopf, M.G., and Nicoll, R. a (1995). A comparison of the role of dynorphin in the hippocampal mossy fiber pathway in guinea pig and rat. J. Neurosci. 15, 6939-6945.

Salin, P.A., Scanziani, M., Malenka, R.C., and Nicoll, R.A. (1996). Distinct short-term plasticity at two excitatory synapses in the hippocampus. Proc. Natl. Acad. Sci. U. S. A. 93, 1330413309.

Salio, C., Lossi, L., Ferrini, F., and Merighi, A. (2006). Neuropeptides as synaptic transmitters. Cell Tissue Res. 326, 583-598.

Sans, N., Petralia, R.S., Wang, Y.-X., li, J.B., Hell, J.W., and Wenthold, R.J. (1996). A Developmental Change in NMDA Receptor-Associated Proteins at Hippocampal Synapses. J. Neurosci. 20, 1260-1271.

Sätzler, K., Söhl, L.F., Bollmann, J.H., Borst, J.G.G., Frotscher, M., Sakmann, B., and Lübke, J.H.R. (2002). Three-dimensional reconstruction of a calyx of Held and its postsynaptic principal neuron in the medial nucleus of the trapezoid body. J. Neurosci. 22, 10567-10579.

Schaffer, M., Pfeffer, S., Mahamid, J., Kleindiek, S., Laugks, T., Albert, S., Engel, B.D., Rummel, A., Smith, A.J., Baumeister, W., et al. (2019). A cryo-FIB lift-out technique enables molecularresolution cryo-ET within native Caenorhabditis elegans tissue. Nat. Methods 16, 757-762.

Scheuber, A., Rudge, R., Danglot, L., Raposo, G., Binz, T., Poncer, J., and Galli, T. (2006). Loss of AP-3 function affects spontaneous and evoked release at hippocampal mossy fiber synapses. Proc. Natl. Acad. Sci. U. S. A. 103, 16562-16567.

Schiavo, G.G., Benfenati, F., Poulain, B., Rossetto, O., De Laureto, P.P., Dasgupta, B.R., and Montecucco, C. (1992). Tetanus and botulinum-B neurotoxins block neurotransmitter release 
by proteolytic cleavage of synaptobrevin. Nature 359, 832-835.

Schiess, A.R.B., Scullin, C., and Partridge, L.D. (2010). Maturation of Schaffer collateral synapses generates a phenotype of unreliable basal evoked release and very reliable facilitated release. Eur. J. Neurosci. 31, 1377-1387.

Schikorski, T., and Stevens, C.F. (1997). Quantitative ultrastructural analysis of hippocampal excitatory synapses. J. Neurosci. 17, 5858-5867.

Schikorski, T., and Stevens, C.F. (2001). Morphological correlates of functionally defined synaptic vesicle populations. Nat. Neurosci. 4, 391-395.

Schmitz, D., Mellor, J., Breustedt, J., and Nicoll, R.A. (2003). Presynaptic kainate receptors impart an associative property to hippocampal mossy fiber long-term potentiation. Nat. Neurosci. 6, 1058-1063.

Schneggenburger, R., Meyer, A.C., and Neher, E. (1999). Released fraction and total size of a pool of immediately available transmitter quanta at a calyx synapse. Neuron 23, 399-409.

Schoch, S., Deák, F., Königstorfer, A., Mozhayeva, M., Sara, Y., Südhof, T.C., and Kavalali, E.T. (2001). SNARE function analyzed in synaptobrevin/VAMP knockout mice. Science 294, 11171122.

Schrod, N., Vanhecke, D., Laugks, U., Stein, V., Fukuda, Y., Schaffer, M., Baumeister, W., and Lucic, V. (2018). Pleomorphic linkers as ubiquitous structural organizers of vesicles in axons. PLoS One 13, 1-17.

Seamon, K.B., Daly, J.W., Metzger, H., de Souza, N.J., and Reden, J. (1983). Structure-Activity Relationships for Activation of Adenylate Cyclase by the Diterpene Forskolin and Its Derivatives. J. Med. Chem. 26, 436-439.

Seino, S., and Shibasaki, T. (2005). PKA-dependent and PKA-independent pathways for CAMPregulated exocytosis. Physiol. Rev. 85, 1303-1342.

Shapira, M., Zhai, R.G., Dresbach, T., Bresler, T., Torres, V.I., Gundelfinger, E.D., Ziv, N.E., and Garner, C.C. (2003). Unitary assembly of presynaptic active zones from Piccolo-Bassoon transport vesicles. Neuron 38, 237-252.

Shepherd, G.M.G., and Harris, K.M. (1998). Three-dimensional structure and composition of CA3 $\rightarrow$ CA1 axons in rat hippocampal slices: Implications for presynaptic connectivity and compartmentalization. J. Neurosci. 18, 8300-8310.

Sherwood, N.T., and Lo, D.C. (1999). Long-term enhancement of central synaptic transmission by chronic brain- derived neurotrophic factor treatment. J. Neurosci. 19, 7025-7036.

Shibasaki, T., Takahashi, H., Miki, T., Sunaga, Y., Matsumura, K., Yamanaka, M., Zhang, C., Tamamoto, A., Satoh, T., Miyazaki, J.I., et al. (2007). Essential role of Epac2/Rap1 signaling in regulation of insulin granule dynamics by cAMP. Proc. Natl. Acad. Sci. U. S. A. 104, 1933319338.

Shin, W., Ge, L., Arpino, G., Villarreal, S.A., Hamid, E., Liu, H., Zhao, W.D., Wen, P.J., Chiang, H.C., and Wu, L.G. (2018). Visualization of Membrane Pore in Live Cells Reveals a DynamicPore Theory Governing Fusion and Endocytosis. Cell 173, 934-945.e12.

Sigler, A., Oh, W.C., Imig, C., Altas, B., Kawabe, H., Cooper, B.H., Kwon, H.B., Rhee, J.S., and Brose, N. (2017). Formation and Maintenance of Functional Spines in the Absence of 
Presynaptic Glutamate Release. Neuron 94, 304-311.e4.

Siksou, L., Varoqueaux, F., Pascual, O., Triller, A., Brose, N., and Marty, S. (2009a). A common molecular basis for membrane docking and functional priming of synaptic vesicles. Eur. J. Neurosci. 30, 49-56.

Siksou, L., Triller, A., and Marty, S. (2009b). An emerging view of presynaptic structure from electron microscopic studies. J. Neurochem. 108, 1336-1342.

Simmons, M.L., and Chavkin, C. (1996). k-Opioid receptor activation of a dendrotoxinsensitive potassium channel mediates presynaptic inhibition of mossy fiber neurotransmitter release. Mol. Pharmacol. 50, 80-85.

De Simoni, A., Griesinger, C.B., and Edwards, F.A. (2003). Development of rat CA1 neurones in acute versus organotypic slices: Role of experience in synaptic morphology and activity. J. Physiol. 550, 135-147.

Smith, J.E., and Reese, T.S. (1980). Use of aldehyde fixatives to determine the rate of synaptic transmitter release. J. Exp. Biol. 89, 19-29.

Smith, H.L., Bourne, J.N., Cao, G., Chirillo, M.A., Ostroff, L.E., Watson, D.J., and Harris, K.M. (2016). Mitochondrial support of persistent presynaptic vesicle mobilization with agedependent synaptic growth after LTP. Elife 5, 1-30.

Sollner, T., Whiteheart, S.W., Brunner, M., Erdjument-Bromage, H., Geromanos, S., Tempst, P., and Rothman, J.E. (1993). SNAP receptors implicated in vesicle targeting and fusion. Nature $362,318-323$.

Sorra, K.E., Mishra, A., Kirov, S.A., and Harris, K.M. (2006). Dense core vesicles resemble active-zone transport vesicles and are diminished following synaptogenesis in mature hippocampal slices. Neuroscience 141, 2097-2106.

Sosinsky, G.E., Crum, J., Jones, Y.Z., Lanman, J., Smarr, B., Terada, M., Martone, M.E., Deerinck, T.J., Johnson, J.E., and Ellisman, M.H. (2008). The combination of chemical fixation procedures with high pressure freezing and freeze substitution preserves highly labile tissue ultrastructure for electron tomography applications. J. Struct. Biol. 161, 359-371.

Spacek, J., and Harris, K.M. (1997). Three-dimensional organization of smooth endoplasmic reticulum in hippocampal CA1 dendrites and dendritic spines of the immature and mature rat. J. Neurosci. 17, 190-203.

Spacek, J., and Harris, K.M. (1998). Three-dimensional organization of cell adhesion junctions at synapses and dendritic spines in area CA1 of the rat hippocampus. J. Comp. Neurol. 393, 58-68.

Start, R.D., Layton, C.M., Cross, S.S., and Smith, J.H. (1992). Reassessment of the rate of fixative diffusion. J. Clin. Pathol. 45, 1120-1121.

Steuer Costa, W., Yu, S. chieh, Liewald, J.F., and Gottschalk, A. (2017). Fast cAMP Modulation of Neurotransmission via Neuropeptide Signals and Vesicle Loading. Curr. Biol. 27, 495-507.

Stevens, C.F., and Tsujimoto, T. (1995). Estimates for the pool size of releasable quanta at a single central synapse and for the time required to refill the pool. Proc. Natl. Acad. Sci. U. S. A. 92, 846-849.

Stigloher, C., Zhan, H., Zhen, M., Richmond, J., and Bessereau, J.L. (2011). The presynaptic 
dense projection of the Caenorhabiditis elegans cholinergic neuromuscular junction localizes synaptic vesicles at the active zone through SYD-2/liprin and UNC-10/RIM-dependent interactions. J. Neurosci. 31, 4388-4396.

Stoppini, L., Buchs, P.-A., and Muller, D. (1991). A simple method for organotypic cultures of nervous tissue. J. Neurosci. Methods 37, 173-182.

Studer, D., Zhao, S., Chai, X., Jonas, P., Graber, W., Nestel, S., and Frotscher, M. (2014). Capture of activity-induced ultrastructural changes at synapses by high-pressure freezing of brain tissue. Nat. Protoc. 9, 1480-1495.

Südhof, T.C. (2002). Synaptotagmins: Why so many? J. Biol. Chem. 277, 7629-7632.

Südhof, T.C. (2012). The presynaptic active zone. Neuron 75, 11-25.

Südhof, T.C., and Rizo, J. (2011). Synaptic vesicle exocytosis. Cold Spring Harb. Perspect. Biol. 3.

Suzuki, E., and Okada, T. (2007). Regional differences in GABAergic modulation for TEAinduced synaptic plasticity in rat hippocampal CA1, CA3 and dentate gyrus. Neurosci. Res. 59, 183-190.

Suzuki, E., and Okada, T. (2008). TEA-induced long-term potentiation at hippocampal mossy fiber-CA3 synapses: Characteristics of its induction and expression. Brain Res. 1247, 21-27.

Tao-Cheng, J.H. (2007). Ultrastructural localization of active zone and synaptic vesicle proteins in a preassembled multi-vesicle transport aggregate. Neuroscience 150, 575-584.

Tao, C.-L., Liu, Y.-T., Zhou, Z.H., Lau, P.-M., and Bi, G.-Q. (2018a). Accumulation of Dense Core Vesicles in Hippocampal Synapses Following Chronic Inactivity. Front. Neuroanat. 12, 1-11.

Tao, C.L., Liu, Y.T., Sun, R., Zhang, B., Qi, L., Shivakoti, S., Tian, C.L., Zhang, P., Lau, P.M., Hong Zhou, Z., et al. (2018b). Differentiation and characterization of excitatory and inhibitory synapses by cryo-electron tomography and correlative microscopy. J. Neurosci. 38, 14931510.

Thanawala, M.S., and Regehr, W.G. (2013). Presynaptic calcium influx controls neurotransmitter release in part by regulating the effective size of the readily releasable pool. J. Neurosci. 33, 4625-4633.

Toth, K., Suares, G., Lawrence, J.J., Philips-Tansey, E., and McBain, C.J. (2000). Differential mechanisms of transmission at three types of mossy fiber synapse. J. Neurosci. 20, 82798289.

Triller, A., and Korn, H. (1985). Activity-dependent deformations of presynaptic grids at central synapses. J. Neurocytol. 14, 177-192.

Trudeau, L.E., Emery, D.G., and Haydon, P.G. (1996). Direct modulation of the secretory machinery underlies PKA-dependent synaptic facilitation in hippocampal neurons. Neuron 17, 789-797.

Tzounopoulos, T., Janz, R., Südhof, T.C., Nicoll, R.A., and Malenka, R.C. (1998). A Role for cAMP in Long-Term Depression at Hippocampal Mossy Fiber Synapses. Neuron 21, 837-845.

Uchinozo, K. (1965). Characteristics of excitatory and inhibitory synapses in the central nervous system of the cat. Nature $207,642-643$. 
Varoqueaux, F., Sigler, A., Rhee, J., Brose, N., Enk, C., Reim, K., and Rosenmund, C. (2002). Total arrest of spontaneous and evoked synaptic transmission but normal synaptogenesis in the absence of Munc13-mediated vesicle priming. PNAS 99, 9037-9042.

Varoqueaux, F., Sons, M.S., Plomp, J.J., and Brose, N. (2005). Aberrant morphology and residual transmitter release at the Munc13-deficient mouse neuromuscular synapse. Mol. Cell. Biol. 25, 5973-5984.

Verhage, M., and Sørensen, J.B. (2008). Vesicle docking in regulated exocytosis. Traffic 9, 1414-1424.

Verhage, M., McMahon, H.T., Ghijsen, W.E.J.M., Boomsma, F., Scholten, G., Wiegant, V.M., and Nicholls, D.G. (1991). Differential release of amino acids, neuropeptides, and catecholamines from isolated nerve terminals. Neuron 6, 517-524.

Villacres, E.C., Wong, S.T., Chavkin, C., and Storm, D.R. (1998). Type I adenylyl cyclase mutant mice have impaired mossy fiber long-term potentiation. J. Neurosci. 18, 3186-3194.

Vogl, C., Cooper, B.H., Neef, J., Wojcik, S.M., Reim, K., Reisinger, E., Brose, N., Rhee, J.S., Moser, T., and Wichmann, C. (2015). Unconventional molecular regulation of synaptic vesicle replenishment in cochlear inner hair cells. J. Cell Sci. 128, 638-644.

Vyleta, N.P., and Jonas, P. (2014). Loose coupling between $\mathrm{Ca}^{2+}$ channels and release sensors at a plastic hippocampal synapse. Science 343, 665-670.

Vyleta, N.P., Borges-Merjane, C., and Jonas, P. (2016). Plasticity-dependent, full detonation at hippocampal mossy fiber-CA3 pyramidal neuron synapses. Elife 5, 1-12.

Wang, S.S.H., Held, R.G., Wong, M.Y., Liu, C., Karakhanyan, A., Kaeser, P.S., Wang, S.S.H., Held, R.G., Wong, M.Y., Liu, C., et al. (2016). Fusion Competent Synaptic Vesicles Persist upon Active Zone Disruption and Loss of Vesicle Docking Article Fusion Competent Synaptic Vesicles Persist upon Active Zone Disruption and Loss of Vesicle Docking. Neuron 91, 777-791.

Washbourne, P., Thompson, P.M., Carta, M., Costa, E.T., Mathews, J.R., Lopez-Benditó, G., Molnár, Z., Becher, M.W., Valenzuela, C.F., Partridge, L.D., et al. (2002). Genetic ablation of the t-SNARE SNAP-25 distinguishes mechanisms of neuroexocytosis. Nat. Neurosci. 5, 19-26.

Watanabe, S., Rost, B.R., Camacho-Pérez, M., Davis, M.W., Söhl-Kielczynski, B., Rosenmund, C., and Jorgensen, E.M. (2013a). Ultrafast endocytosis at mouse hippocampal synapses. Nature 504, 242-247.

Watanabe, S., Liu, Q., Davis, M.W., Hollopeter, G., Thomas, N., Jorgensen, N.B., and Jorgensen, E.M. (2013b). Ultrafast endocytosis at Caenorhabditis elegans neuromuscular junctions. Elife 2013, 1-24.

Weimer, R.M., Gracheva, E.O., Meyrignac, O., Miller, K.G., Richmond, J.E., and Bessereau, J. (2006). UNC-13 and UNC-10 Rim Localize Synaptic Vesicles to Specific Membrane Domains. J. Neurosci. 26, 8040-8047.

Weisskopf, M., Castillo, P., Zalutsky, R., and Nicoll, R. (1994). Mediation of hippocampal mossy fiber long-term potentiation by cyclic AMP. Science 265, 1878-1882.

Weisskopf, M.G., Zalutsky, R.A., and Nicoll, R.A. (1993). The opioid peptide dynorphin mediates heterosynaptic depression of hippocampal mossy fibre synapses and modulates long-term potentiation. Nature 365, 188-188. 
Wilke, S.A., Antonios, J.K., Bushong, E.A., Badkoobehi, A., Malek, E., Hwang, M., Terada, M., Ellisman, M.H., and Ghosh, A. (2013). Deconstructing Complexity : Serial Block-Face Electron Microscopic Analysis of the Hippocampal Mossy Fiber Synapse. J. Neurosci. 33, 507-522.

Xia, Z., Refsdal, C.D., Merchant, K.M., Dorsa, D.M., and Storm, D.R. (1991). Distribution of mRNA for the calmodulin-sensitive adenylate cyclase in rat brain: Expression in areas associated with learning and memory. Neuron 6, 431-443.

Xu-Friedman, M.A., and Regehr, W.G. (2003). Ultrastructural contributions to desensitization at cerebellar mossy fiber to granule cell synapses. J. Neurosci. 23, 2182-2192.

Xu-Friedman, M.A., Harris, K.M., and Regehr, W.G. (2001). Three-Dimensional Comparison of Ultrastructural Characteristics at Depressing and Facilitating Synapses onto Cerebellar Purkinje Cells. J. Neurosci. 21, 6666-6672.

Yamamoto, C., Sawada, S., and Ohno-Shosaku, T. (1993). Quantal analysis of modulating action of adenosine on the mossy fiber synapse in hippocampal slices. Hippocampus 3, 8792.

Yang, Y., and Calakos, N. (2011). Munc13-1 Is Required for Presynaptic Long-Term Potentiation. J. Neurosci. 31, 12053-12057.

Zalutsky, R.A., and Nicoll, R.A. (1990). Comparison of two forms of long-term potentiation in single hippocampal neurons. Science 248, 1619-1624.

Zhao, S., Studer, D., Chai, X., Graber, W., Brose, N., Nestel, S., Young, C., Rodriguez, E.P., Saetzler, K., and Frotscher, M. (2012a). Structural plasticity of spines at giant mossy fiber synapses. Front. Neural Circuits 6, 103.

Zhao, S., Studer, D., Graber, W., Nestel, S., and Frotscher, M. (2012b). Fine structure of hippocampal mossy fiber synapses following rapid high-pressure freezing. Epilepsia 53, 4-8.

Zhao, S., Studer, D., Chai, X., Graber, W., Brose, N., Nestel, S., Young, C., Rodriguez, E.P., Saetzler, K., and Frotscher, M. (2012c). Structural plasticity of hippocampal mossy fiber synapses as revealed by high-pressure freezing. J. Comp. Neurol. 520, 2340-2351.

Zuber, B., and Lučić, V. (2019). Molecular architecture of the presynaptic terminal. Curr. Opin. Struct. Biol. 54, 129-138. 


\section{Supplemental Figures and Tables}

\subsection{Perfusion fixation changes the localization of DCVs in mossy fiber synapses}

DCVs were observed in perfusion-fixed mossy fiber synapses from P28 wild-type animals. Two perfusion protocols from key morphological studies of hippocampal MF boutons were used to determine whether chemical fixation changed the membrane-proximal pools of synaptic vesicles. The first perfusion fixation of wild-type mice at P28 were as follows: Perfusion Fixative 1 (PF1), ice-cold 4\% PFA, 2.5\% GA in 0.1 M PB, or Perfusion Fixative 2 (PF2) consisted of $2 \%$ PFA, $2.5 \% \mathrm{GA}, 2 \mathrm{mM} \mathrm{CaCl} 2$ in $0.1 \mathrm{M}$ cacodylate buffer at $37^{\circ} \mathrm{C}$. DCVs were observed within $100 \mathrm{~nm}$ of the active zone membrane in mossy fiber synapses from both perfusion fixation protocols, PF1 and PF2 (Figure 20 A). Synapses fixed with PF2 had a higher density of docked DCVs than untreated mossy fiber synapses from DIV28 slice culture while mossy fiber synapses from PF1 had no docked DCVs (Figure 20 B, $0 \pm 0$, PF1; $0.141 \pm 0.043$, PF2; $p=0.001$; Figure 18 B, DIV28 slice culture). However, DCVs accumulated within $40 \mathrm{~nm}$ of the active zone in both PF1 and PF2 mossy fiber synapses (Figure 20 C; $0.195 \pm 0.049, \mathrm{PF} 1 ; 0.184 \pm 0.057, \mathrm{PF} 2$; $\mathrm{p}=0.62$ ). While some additional DCVs were observed beyond $40 \mathrm{~nm}$ of the active zone in conditions (Figure $20 \mathrm{D} ; 0.241 \pm 0.049, \mathrm{PF} 1 ; 0.255 \pm 0.057$, PF2; $p=0.94$ ), there appeared to be a shift in DCVs toward the active zone membrane with no further recruitment of DCVs indicating potential fusion of DCVs and a mobilization of membrane-proximal DCVs (Figure 20 A).
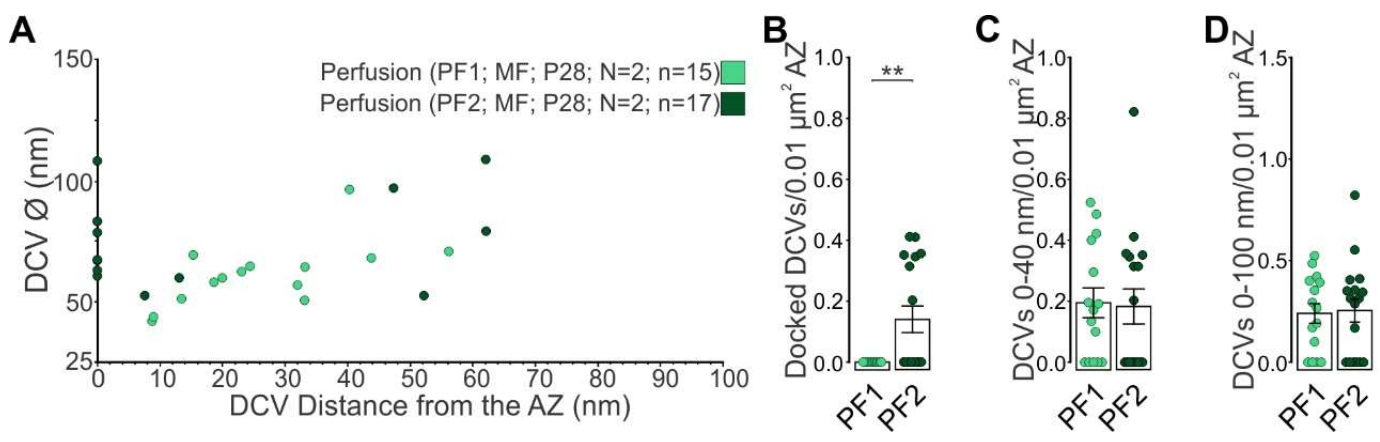

Figure 20. Spatial distribution of DCVs in mossy fiber-CA3 synapses in perfusion-fixed ex vivo preparations.

(A) Diameters of all DCVs analyzed and their respective distance to the active zone membrane. (B) Scatter plot of the number of docked DCVs normalized to active zone area. (C, D) Scatter plots of the number of DCVs within $40 \mathrm{~nm}$ (C) and $100 \mathrm{~nm}$ (D) of the active zone membrane normalized to active zone area. Statistical significance 
is represented as $*, \mathrm{p}<0.05 ;{ }^{* *}, \mathrm{p}<0.005 ;{ }^{* *}, \mathrm{p}<0.001 . \mathrm{N}=$ number of animals, $\mathrm{n}=$ number of active zones. See Tables 21-22 for full statistical analyses. 


\subsection{The effect of aldehydes on synaptic vesicle docking is depth- dependent in immersion-fixed hippocampal slice culture}

To test the direct effect of aldehydes on synaptic vesicle docking, I immersion-fixed slice cultures with the same fixation solutions as the perfusion fixation experiment. The immersion-fixation of hippocampal slice cultures at DIV28 were as follows: Immersion Fixative 1 (IF1; Figure 21 A), ice-cold 4\% PFA, 2.5\% GA in 0.1 M PB overnight, or Immersion Fixative 2 (IF2; Figure $21 \mathrm{~B}$ ), 2\% PFA, $2.5 \% \mathrm{GA}, 2 \mathrm{mM} \mathrm{CaCl}_{2}$ in $0.1 \mathrm{M}$ cacodylate buffer initially at $37^{\circ} \mathrm{C}$, then cooled to room temperature for one hour before overnight incubation at $4^{\circ} \mathrm{C}$. Immersion-fixed slices (DIV28) and untreated control slices from the same culture (DIV29) were prepared for comparative ultrastructural analysis by HPF and AFS. The age discrepancy between IF1/2 and cryo-fixed control was due to the overnight incubation of the immersionfixed slices in their respective aldehyde cocktails before HPF. Vesicle docking at mossy fiber active zones was initially examined in plastic sections acquired at depths of 5-11 $\mu \mathrm{m}$ from the surface of the tissue, a depth at which mossy fiber and Schaffer collateral synapses are reliably located and very well cryo-preserved. In these superficial regions, the density of docked synaptic vesicles quantified for both immersion fixation protocols was highly comparable to directly cryo-fixed controls (Figure 21 C; $1.135 \pm 0.111$, HFP; $1.065 \pm 0.121$, IF1; $1.373 \pm 0.152$, IF2). The diameter of docked synaptic vesicles was unchanged by immersion fixation (Figure 21 D; $52.11 \pm 0.805$ nm, HPF; $51.34 \pm 1.085$ nm, IF1, $50.60 \pm 0.689$ nm, IF2; p>0.05). Comparable numbers of docked giant vesicles were quantified in all three conditions (Figure $21 \mathrm{E} ; 0.086 \pm 0.034$, HPF; $0.122 \pm 0.043$, IF1; $0.068 \pm 0.035, I F 2 ; p>0.05$ ).

Since aldehyde fixation is diffusion-limited (Hopwood, 1969; Start et al., 1992), the rate of fixation is inversely proportional to tissue depth (Start et al., 1992). Therefore, I also examined vesicle docking at mossy fiber active zones in synapses located deeper below the slice surface. 

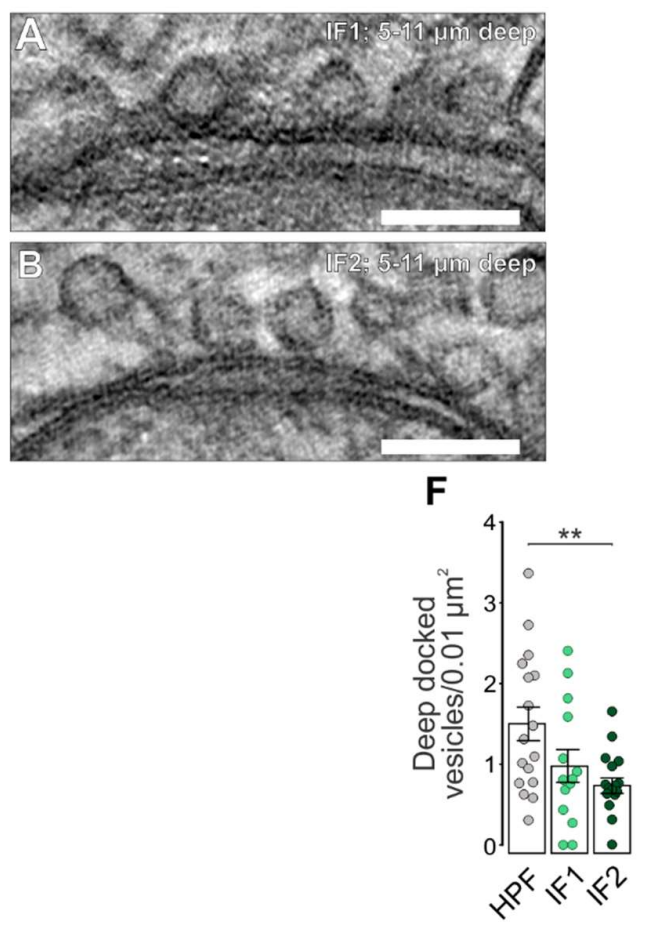

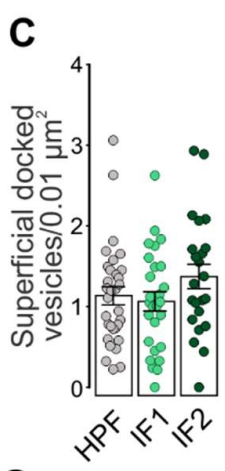

\section{G}

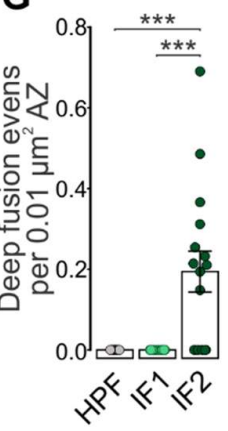

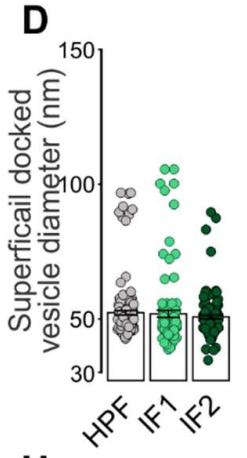

H

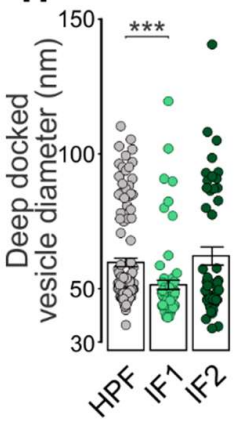

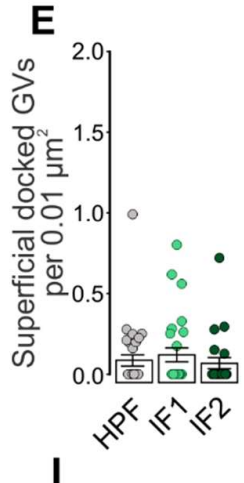

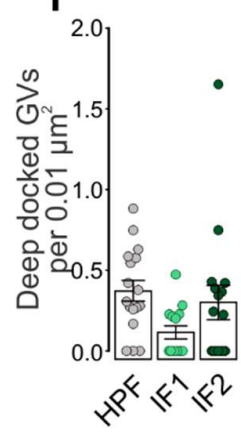

Figure 21. Ultrastructural analysis of docked synaptic vesicles at mossy fiber synapses from immersion-fixed slice cultures at DIV28.

(A, B) Tomographic subvolumes of mossy fiber synapses from immersion-fixed slice cultures at DIV28. The two immersion aldehyde cocktails were: Immersion Fixative 1 (IF1) comprised of 4\% PFA, 2.5\% GA in $0.1 \mathrm{M}$ PB, and Immersion Fixative 2 (IF2) composed of $2 \%$ PFA, $2.5 \% \mathrm{GA}, 2 \mathrm{mM} \mathrm{CaCl} 2$ in $0.1 \mathrm{M}$ cacodylate buffer. (C-E) Ultrastructural analysis of docked vesicles at mossy fiber active zones 5-11 $\mu \mathrm{m}$ from the surface of the tissue (superficial). (F-I) Ultrastructural analysis of docked vesicles at mossy fiber active zones 20-22 $\mu \mathrm{m}$ from the surface of the tissue (deep). (C, F) Scatter plots of the number of docked vesicles normalized to active zone area. (D, H) Scatter plots of the docked vesicle diameter. (E, I) Scatter plots of the number of docked giant vesicles normalized to active zone area. (G) Scatter plot of morphological fusion events normalized to active zone area. Scale bars: $100 \mathrm{~nm}, \mathbf{A}$ and $\mathbf{B}$. Statistical significance is represented as *, $\mathrm{p}<0.05 ; * *, \mathrm{p}<0.005 ; * * *, p<0.001 . \mathrm{N}=3$ cultures, superficial; 2 cultures, deep; superficial $n=32$, HPF; 27, IF1; and 23 active zones, IF2; deep $n=17$, HPF; 14, IF1; and 16 active zones, IF2. See Tables 30 and 31 for superficial $(5-11 \mu \mathrm{m})$ and deeper $(20-22 \mu \mathrm{m})$, respectively for full statistical analyses.

In sections acquired at depths of 20-22 $\mu \mathrm{m}$ below the slice surface, immersion-fixed slices exhibited a strong tendency towards reduced docking compared to directly cryo-fixed controls that reached statistical significance for IF2 (Figure $21 \mathrm{~F} ; 1.503 \pm 0.209$, HPF; $0.981 \pm$ $0.201, \mathrm{IF} 1 ; 0.777 \pm 0.097, \mathrm{IF} 2 ; \mathrm{HPF} \vee \mathrm{IF} 2, \mathrm{p}=0.004)$. Moreover, the observed reduction in docked synaptic vesicle density in the IF2 condition was associated with a concomitant increase in the density of omega-shaped membrane profiles indicative of full-collapse vesicle fusion (Figure $21 \mathrm{G} ; 0 \pm 0$, HPF; $0 \pm 0$, IF1; $0.195 \pm 0.050$, IF2; $p<0.001$ ). The mean docked synaptic vesicle diameter was reduced in IF1 condition compared to directly cryo-fixed 
controls (Figure $21 \mathrm{H}, 59.66 \pm 1.63 \mathrm{~nm}$, HPF; $51.33 \pm 1.74 \mathrm{~nm}$, IF1; $62.02 \pm 3.38 \mathrm{~nm}$, IF2; HPF v IF1 $p<0.001$, IF1 v IF2 $p=0.01$, HPF $\vee$ IF2 $p=0.37$ ).

This finding can be explained by the depletion of docked giant vesicles in the IF1 condition (Figure 21 I; $0.373 \pm 0.063$, HPF; $0.116 \pm$ IF1; $0.302 \pm 0.106$, IF2; HPF v IF1 p=0.003, IF2 v IF1 and HPF p>0.05). In the IF2 condition, the density of docked giant vesicles was highly comparable to controls, despite the observed depletion of smaller "normal" sized synaptic vesicles (Figure $21 \mathrm{~F} ; 1.503 \pm 0.209, \mathrm{HPF} ; 0.981 \pm 0.201$, IF1; $0.777 \pm 0.097$, IF2; HPF v IF2, $p=0.004)$.

It is likely that the IF1 protocol, due to the temperature of the fixative, slowed the dynamic cellular processes at the mossy fiber synapse enough to occlude the effects of aldehyde fixation on docked synaptic vesicles, while the physiological temperature of IF2 captured the detrimental morphological effects of aldehydes at the mossy fiber synapse. 


\subsection{Presynaptic structural parameters are essentially unaffected by acute pharmacological manipulation of cAMP in hippocampal mossy fiber boutons}

Acute pharmacological treatment of cultured hippocampal slices with either TEA, a potassium channel blocker (Aniksztejn and Ben-Ari, 1991) or forskolin, an AC1 activator result in longterm changes in mossy fiber synaptic strength (Barovsky et al., 1984; Suzuki and Okada, 2007, 2008; Weisskopf et al., 1994). Whereas TEA causes a broadening of the AP and allows a longer activation of VGCCs, and increased calcium influx (Suzuki and Okada, 2008; Zhao et al., 2012c, 2012a), forskolin-induced increases in presynaptic cAMP production are thought to trigger a signaling cascade involving PKA (Evans and Morgan, 2003; Seino and Shibasaki, 2005; Steuer Costa et al., 2017; Trudeau et al., 1996; Villacres et al., 1998), although the downstream effectors of PKA and their effects on numbers of docked and primed vesicles remain largely unknown. Acute TEA treatment was previously demonstrated to induce structural changes during mossy fiber potentiation, including an increase in the ratio of bouton perimeter and area, increases in the number of postsynaptic spines, and an increase in the number of active zones normalized to bouton perimeter (Zhao et al., 2012c, 2012a).

I tested whether increasing presynaptic cAMP production with forskolin increased the number of active zones in mossy fiber boutons. To achieve this, STED microscopy was performed on hippocampal slice cultures after a 15-minute treatment with either VC or T/F prior to fixation with the help of Sinem Sertel. The synaptic vesicle protein, synaptoporin was used to label synaptic vesicle clusters in mossy fiber boutons (Figure22 A and C). Bassoon was used as an active zone marker (Figure $22 \mathrm{~B}$ and C). I found that there was no change in the amount of bassoon-labelled active zones after T/F treatment (Figure $22 \mathrm{E}$ ).

To test whether ultrastructural changes are induced in response to acute forskolin treatment, I systematically analyzed and compared presynaptic ultrastructural parameters in hippocampal mossy fiber synapses in two conditions: T/F and VC. After a 15-minute incubation in the respective drug cocktails, slices were quickly fixed in ice-cold 4\% PFA. Image acquisition and analysis was performed blind with the help of Kirsten Weyand. In ultra-thin sections, 3Dmod software was used to segment the perimeter of presynaptic boutons, presynaptic mitochondria, active zones, and postsynaptic spine compartments (Figure 22 D) (Kremer et al., 1996). Forskolin treatment had almost no appreciable effect on the quantified 
parameters at the time point analyzed. Mossy fiber bouton area and perimeter were comparable between conditions (Figure $22 \mathrm{~F}$ and G; bouton area: $5.053 \pm 0.410 \mu \mathrm{m}^{2}$, VC; $4.969 \pm 0.352 \mu \mathrm{m}^{2}, \mathrm{~T} / \mathrm{F} ; \mathrm{p}=0.42$; perimeter: $13.66 \pm 0.805 \mu \mathrm{m}, \mathrm{VC} ; 12.07 \pm 0.573 \mu \mathrm{m}, \mathrm{T} / \mathrm{F}$; $p=0.31$ ). In contrast to previous observations in TEA-treated slices (Zhao et al., 2012c), the bouton perimeter normalized to bouton area was actually smaller in T/F compared to VC treated slices (Figure $22 \mathrm{H} ; 3.188 \pm 0.108 \mu \mathrm{m}^{-1}$, VC; $2.760 \pm 0.099 \mu \mathrm{m}^{-1}, \mathrm{~T} / \mathrm{F} ; \mathrm{p}=0.002$, The number of active zones normalized to bouton perimeter was comparable between conditions (Figure 22 I; $0.426 \pm 0.026 \mathrm{AZs}$ per $\mu \mathrm{m}, \mathrm{VC} ; 0.419 \pm 0.023 \mathrm{AZs}$ per $\mu \mathrm{m}, \mathrm{T} / \mathrm{F} ; \mathrm{p}=0.84$ ), although a slight reduction in active zone length was observed after T/F treatment (Figure $22 \mathrm{~J} ; 343.2$ $\pm 16.06 \mathrm{~nm}, \mathrm{VC} ; 306.0 \pm 12.68 \mathrm{~nm}, \mathrm{~T} / \mathrm{F} ; \mathrm{p}=0.10)$. The contact length of the presynaptic bouton with postsynaptic spines was also unchanged after T/F treatment (Figure $22 \mathrm{~K} ; 1241 \pm 75.26$ $\mathrm{nm}, \mathrm{VC} ; 1296 \pm 92.65 \mathrm{~nm}, \mathrm{~T} / \mathrm{F} ; \mathrm{p}=0.65)$. Also in contrast to previous observations in TEAtreated slices (Zhao et al., 2012c), I observed fewer thorny spine excrescences per unit area of mossy fiber bouton after T/F treatment (Figure $22 \mathrm{~L} ; 0.738 \pm 0.066$ spines per $\mu \mathrm{m}^{2}$, VC; $0.539 \pm 0.060$ spines per $\left.\mu m^{2}, T / F ; p=0.01\right)$. Despite fewer spines, the mean spine area remained comparable to VC (Figure $22 \mathrm{M} ; 0.434 \pm 0.045 \mu \mathrm{m}^{2}$, VC; $0.470 \pm 0.045 \mu \mathrm{m}^{2}, \mathrm{~T} / \mathrm{F}$; $\mathrm{p}=0.52$ ) as well as the spine area in proportion to mossy fiber bouton area (Figure $22 \mathrm{~N} ; 29.89$ $\pm 2.985 \%, V C ; 27.71 \pm 3.885 \%, T / F ; p=0.14)$.

Synaptic release probability and synaptic strength have also been related to the abundance, morphology, and subcellular location of presynaptic mitochondria (Cserép et al., 2018; Ivannikov et al., 2013; Lee et al., 2012; Nguyen et al., 1997; Perkins et al., 2010; Rangaraju et al., 2014; Smith et al., 2016). To this end, I compared mitochondrial morphology and their respective distances from mossy fiber active zones in T/F and VC treated slices. I found comparable mitochondrial morphologies in mossy fiber synapses treated with VC and T/F by measuring total mitochondrial area (Figure $220 ; 0.340 \pm 0.035, \mathrm{VC} ; 0.359 \pm 0.035, \mathrm{~T} / \mathrm{F}$; $\mathrm{p}=0.51$ ), as well as the ratio between mitochondrial perimeter and area (Figure $22 \mathrm{P} ; 20.21 \pm$ $\left.0.528 \mu^{-1}, V C ; 18.94 \pm 0.446 \mu m^{-1}, T / F ; p=0.26\right)$. Mitochondria were located farther from mossy fiber active zones after T/F treatment (Figure Q M; $488.5 \pm 42.19 \mathrm{~nm}$, VC; $601.0 \pm 43.38$, $T / F ; p=0.04)$. Likely the mechanism of forskolin-induced changes to release probability in mossy fiber synapses occurs independent of mitochondria. 


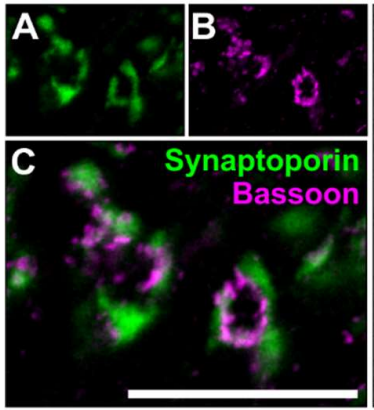

$\mathbf{F}$
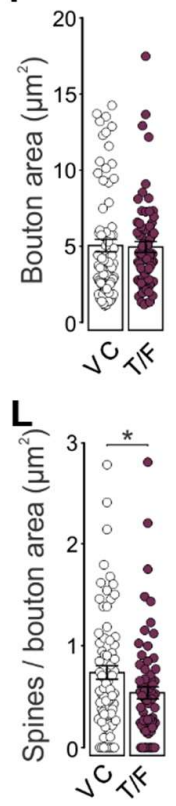

G

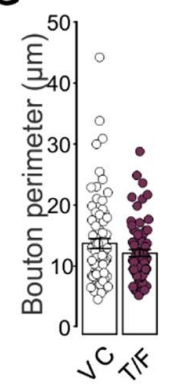

M

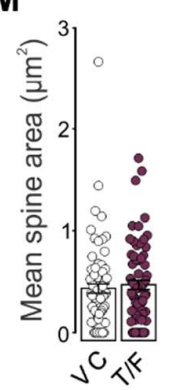

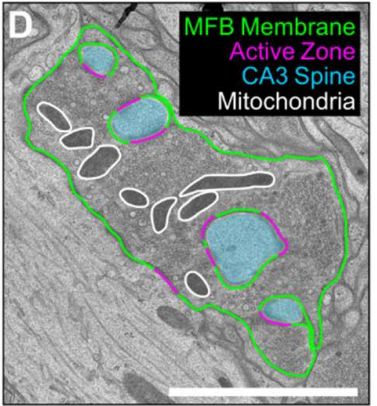

H

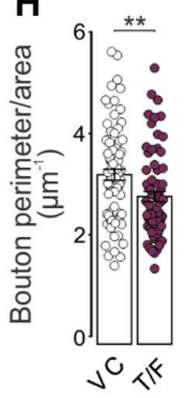

N

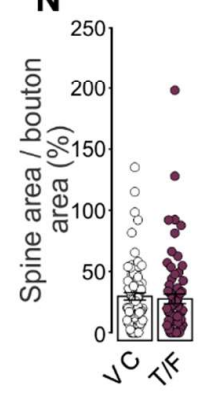

E

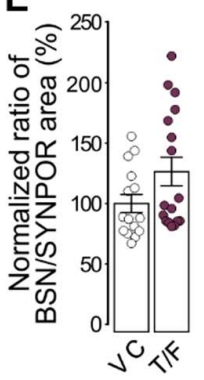

I

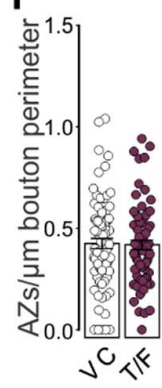

J

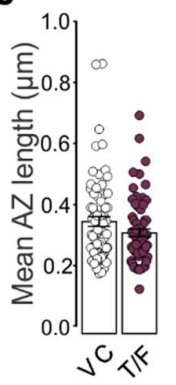

0

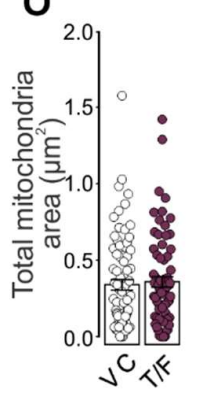

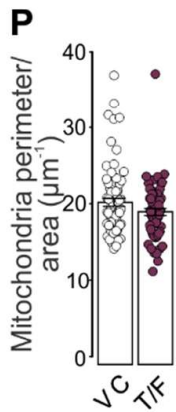

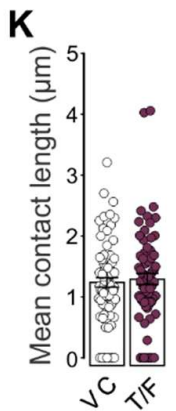

Q

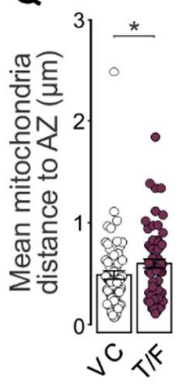

Figure 22. Ultrastructural analysis of mossy fiber boutons after forskolin treatment in slice cultures at DIV28.

(A-C, and E) Two-color STED images for synaptoporin (green) and bassoon (magenta) obtained by immunostaining with the respective primary antibodies and secondary antibodies conjugated to STAR580 and Atto647N, respectively. Bassoon staining that corresponded to each synaptoporin object was determined in mossy fiber boutons after a 15-minute treatment with either VC ( $1 \mu \mathrm{M} \mathrm{TTX)} \mathrm{or} \mathrm{T/F} \mathrm{(} 1 \mu \mathrm{M}$ TTX and $25 \mu \mathrm{M}$ forskolin) (E). (D, F-Q) Ultrastructural 2D analysis of mossy fiber boutons, spines, and mitochondria in slice cultures treated for 15 minutes with VC and T/F at DIV28. (F-H) Scatter plots of mossy fiber bouton parameters, bouton area (F), length of bouton perimeter $(\mathbf{G})$ and the relative length of the bouton perimeter compared to area $(\mathbf{H})$. (I-J) Scatter plots of the number of active zones normalized to bouton perimeter (I) and mean active zone length per bouton (J). (K) Scatter plot of the mean contact length between bouton and spine. (L-N) Scatter plots of postsynaptic spine morphology: number of spines per bouton area (L), mean spine area (M), and spine area relative to bouton area (N). (O-Q) Morphological analysis of presynaptic mitochondria: the total mitochondrial area $(\mathbf{O})$, perimeter relative to mitochondrial area $(\mathbf{P})$, and mean mitochondria distance to the closest active zone (Q). Scale bars: $5 \mu \mathrm{m}, \mathbf{C} ; 2 \mu \mathrm{m}, \mathbf{D}$. Statistical significance is represented as $*, \mathrm{p}<0.05 ; * *, \mathrm{p}<0.005 ; * * *$, $p<0.001$. $N=3$ cultures; $n=77$ boutons, VC; 72 boutons T/F. See Tables 32 and 33 for full statistical analyses of STED imaging and 2D EM imaging, respectively. 
A

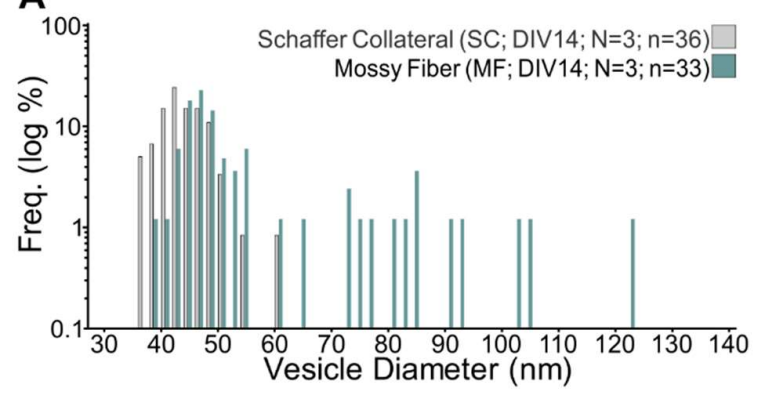

C

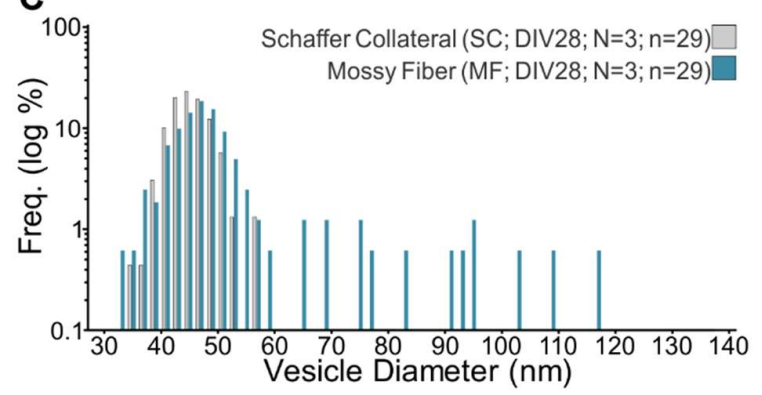

E

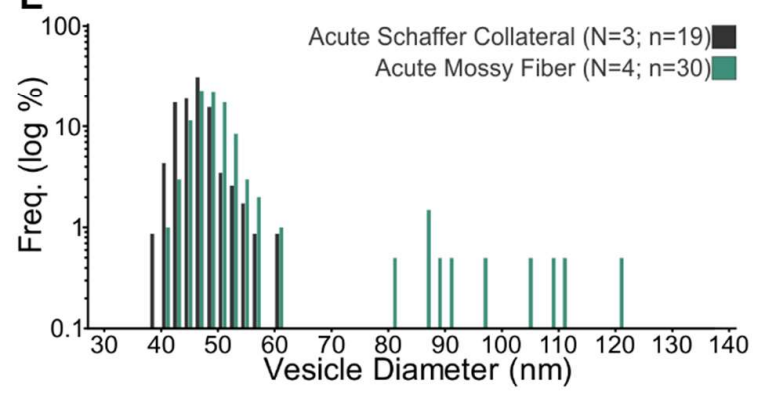

B

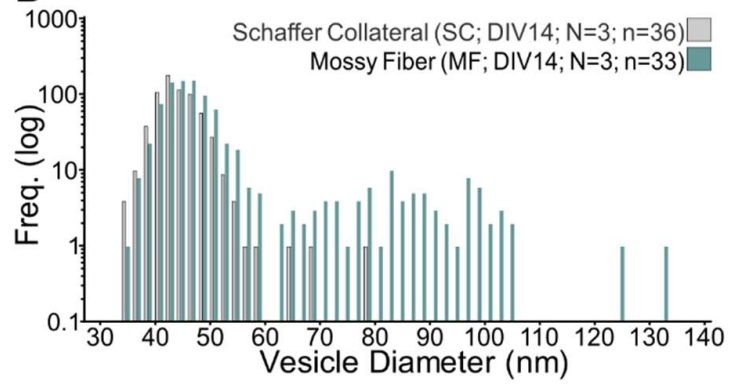

D

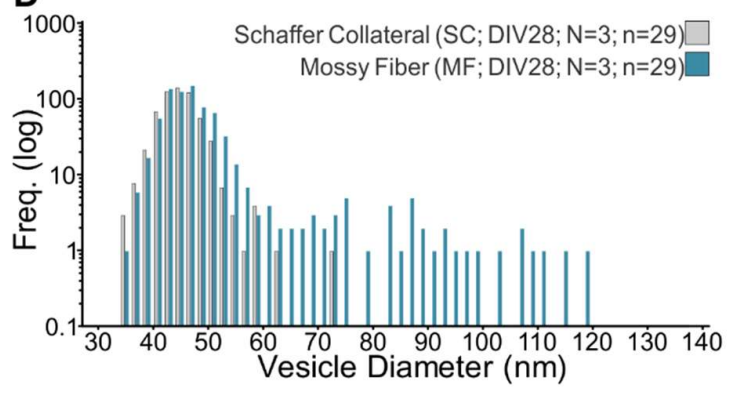

$\mathbf{F}$

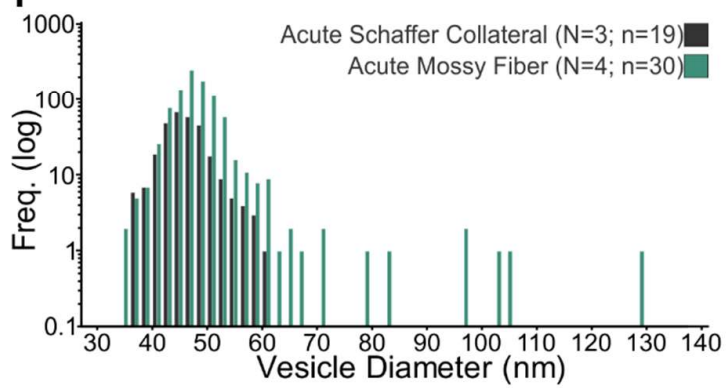

Figure 23. Distributions of vesicle diameter in Schaffer collateral and mossy fiber synapses from acute and cultured slice preparations.

(A-F) Analysis of vesicle diameters in Schaffer collateral synapses from organotypic slice cultures at DIV14 (A-B) and DIV28 (C-D) as well as from acute hippocampal slice preparations from P18 wild-type mice (E-F). (A, C, E) Frequency distribution plots of the docked vesicle diameter represented as percentage. (B, D, F) Frequency distribution plots indicating the diameters of all non-docked vesicles within 0-100 $\mathrm{nm}$ of the active zone and represented as raw numbers. $\mathrm{N}=$ number of cultures, $\mathbf{A}-\mathbf{D}$; number of animals, $\mathbf{E}-\mathbf{F} ; \mathrm{n}=$ number of active zones. 
A

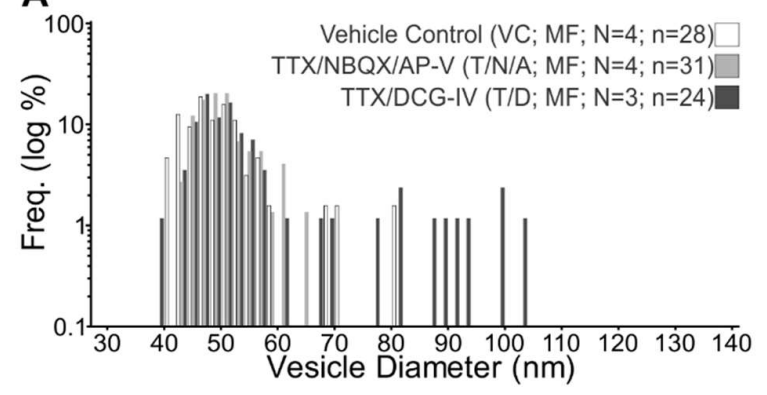

C

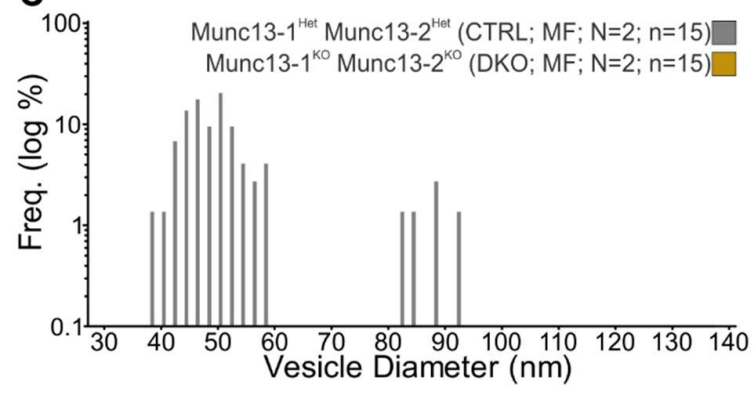

E

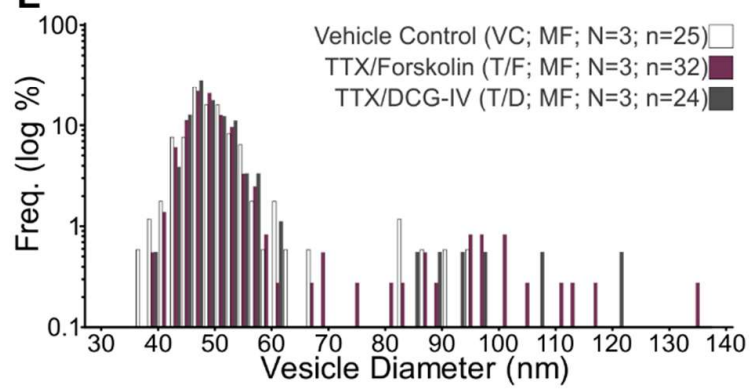

B

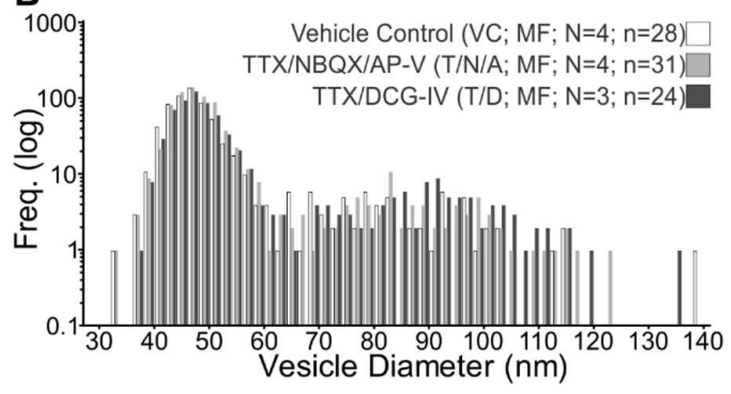

D

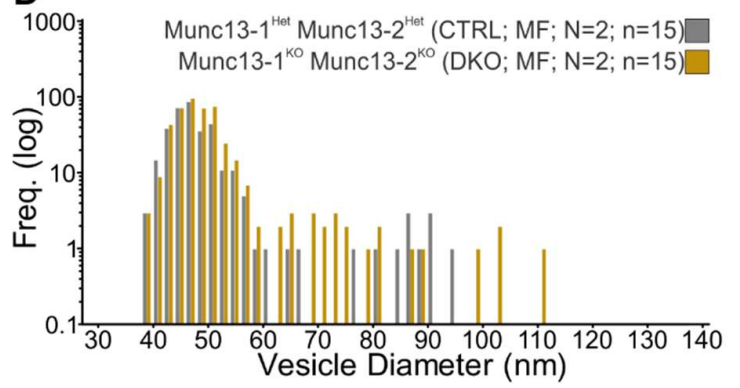

$\mathbf{F}$

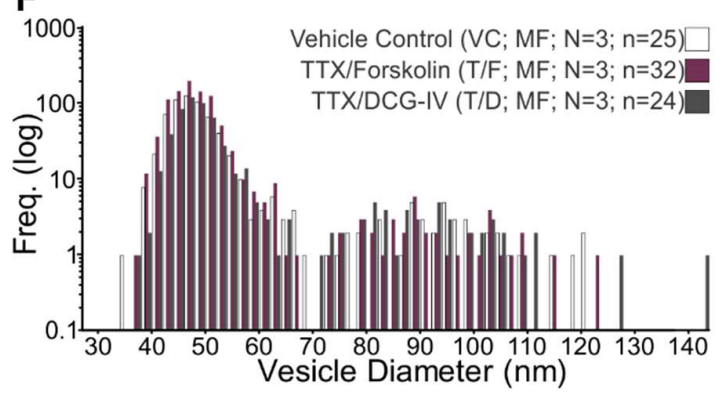

Figure 24. Vesicle diameter distributions in mossy fiber-CA3 synapses after genetic or pharmacological manipulations of synaptic transmission.

(A-B) Analysis of vesicle diameters in mossy fiber synapses after a 10 minute pharmacological treatment of slice cultures at DIV14 with one of three conditions: 1) T/N/A: TTX, to block sodium-propagated action potentials, supplemented with either NBQX and D-AP5 to block AMPA and NMDA receptor blockers, respectively; 2) T/D: TTX with DCG-IV, an mGluR2 receptor agonist that specifically blocks synaptic transmission in mossy fiber synapses (Kamiya and Ozawa, 1999); and 3) VC: a vehicle control comprised of slice culture medium. (C-D) Analysis of vesicle diameters in mossy fiber synapses from Munc13-1/2 DKO and CTRL slice cultures at DIV28. (E-F) Analysis of vesicle diameters in mossy fiber synapses after a 15 minute treatment with either: 1) T/F: TTX, to block sodium propagated action potentials, and forskolin, an activator of AC1 which increases presynaptic cAMP concentrations (Chavez-Noriega and Stevens, 1994; Dixon and Atwood, 1989; Seamon et al., 1983) thus increasing synaptic transmission (Evans and Morgan, 2003; Seino and Shibasaki, 2005; Weisskopf et al., 1994); 2) T/D: TTX with DCG-IV, and mGluR2 receptor agonist that specifically blocks synaptic transmission in mossy fiber synapses (Kamiya and Ozawa, 1999); and 3) VC: a vehicle control comprised of TTX in slice culture medium in hippocampal slice cultures at DIV28. (A, C, E) Frequency distribution plots of the docked vesicle diameter represented as percentage. (B, D, F) Frequency distribution plots of non-docked vesicle diameter represented as raw numbers. $\mathrm{N}=$ number of cultures, $\mathrm{n}=$ number of active zones. 
A
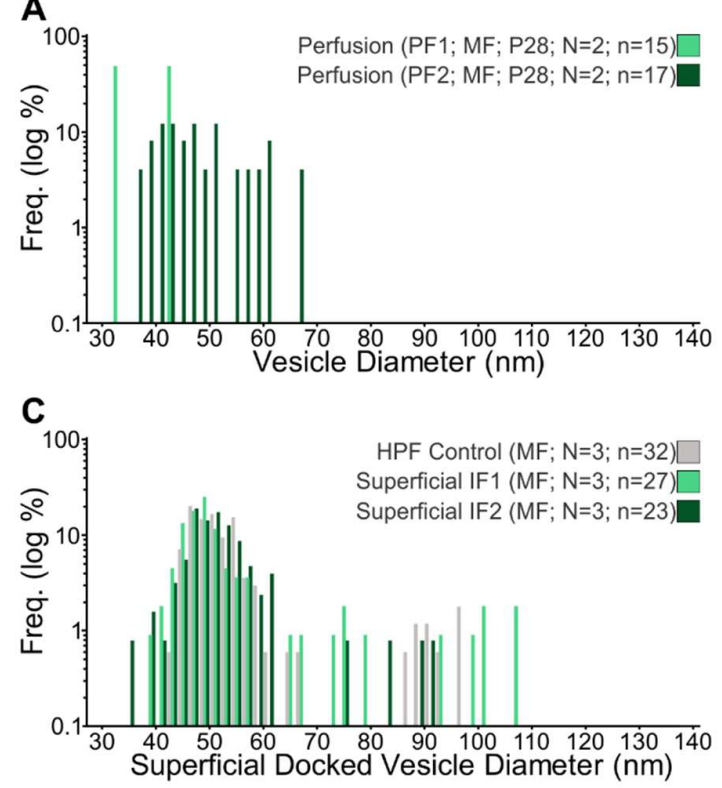
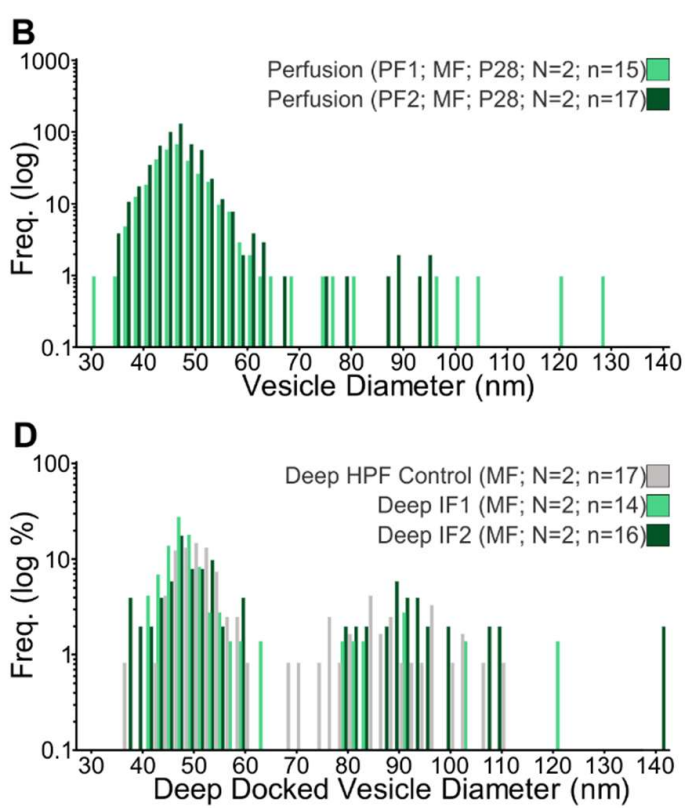

Figure 25. Vesicle diameter distributions in mossy fiber-CA3 synapses from aldehyde-fixed ex vivo preparations and high-pressure frozen organotypic slice cultures.

(A-B) Analysis of vesicle diameters in mossy fiber synapses from P28 wild-type mice perfusion-fixed with either: i) Perfusion Fixative 1 (PF1) comprised of ice-cold 4\% PFA, 2.5\% GA dissolved in in $0.1 \mathrm{M}$ PB; or ii) Perfusion Fixative 2 (PF2) comprised of $2 \%$ PFA, $2.5 \% \mathrm{GA}, 2 \mathrm{mM} \mathrm{CaCl} 2$ dissolved in $0.1 \mathrm{M}$ cacodylate buffer at $37^{\circ} \mathrm{C}$. (C-D) Analysis of docked vesicle diameters from mossy fiber synapses after immersion-fixation of hippocampal slice cultures at DIV28: Immersion Fixative 1 (IF1), ice-cold 4\% PFA, 2.5\% GA in $0.1 \mathrm{M}$ PB overnight, or Immersion Fixative 2 (IF2), $2 \%$ PFA, $2.5 \% \mathrm{GA}, 2 \mathrm{mM} \mathrm{CaCl} 2$ in $0.1 \mathrm{M}$ cacodylate buffer initially at $37^{\circ} \mathrm{C}$, then cooled to room temperature for one hour before overnight incubation at $4^{\circ} \mathrm{C}$. Fresh cryo-fixed slice cultures served as a control (HPF). Docked vesicle analysis of immersion-fixation was performed close to the surface of the tissue (C; Superficial; 5-11 $\mu \mathrm{m}$ into the tissue) and deeper (D; Deep; 20-22 $\mu \mathrm{m}$ into the tissue). (A, C) Frequency distribution plots of the docked vesicle diameter represented as percentage. (B, D) Frequency distribution plots of nondocked vesicle diameter represented as raw numbers. $N=$ number of animals (A-B); $N=$ number of cultures (C-D), $n=$ number of active zones. 

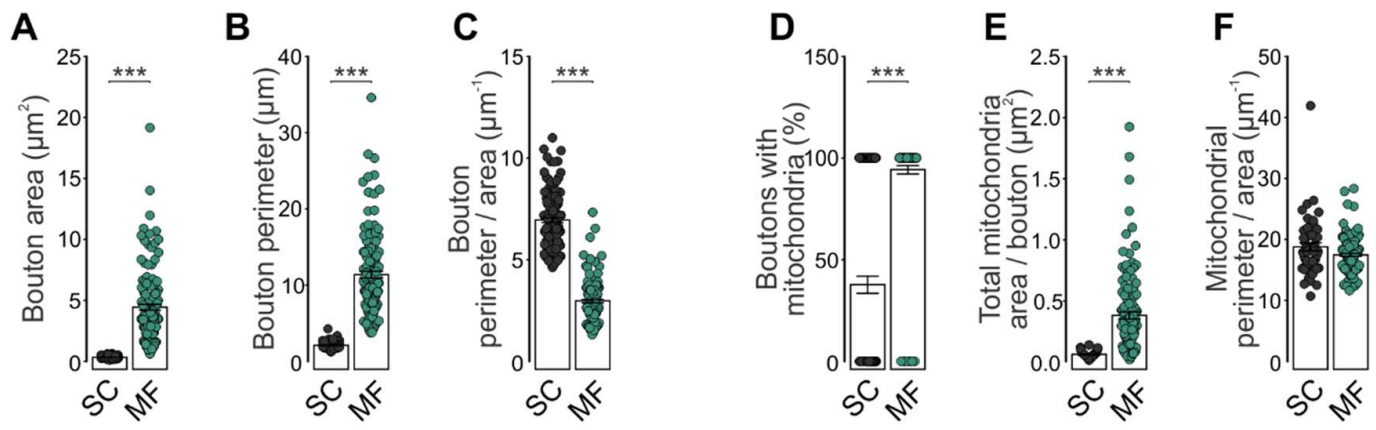

Figure 26. 2D ultrastructural comparison of Schaffer collateral and mossy fiber-CA3 synapses from DIV28 organotypic slice cultures.

(A-C) Scatter plots of presynaptic bouton ultrastructure in Schaffer collateral and mossy fiber synapses; bouton area (A), bouton perimeter (B), and the proportion of bouton perimeter in relation to the area (C). (D-F) Scatter plots of the comparison of presynaptic mitochondria between Schaffer collateral and mossy fiber synapses; the proportion of all boutons analyzed that contained a presynaptic mitochondrion (A), the total mitochondrial area per bouton (E), and the ratio between mitochondrial perimeter and area (F). Statistical significance is represented as *, $\mathrm{p}<0.05 ;{ }^{* *}, \mathrm{p}<0.005 ;{ }^{* * *}, \mathrm{p}<0.001$. N=3 cultures, $\mathrm{n}=138$ boutons (A-D) both SC and MF; $\mathrm{n}=52$ boutons SC and 130 boutons MF (E-F). See Table 34 for full statistical analyses.

\subsection{Mitochondria in Schaffer collateral and mossy fiber synapses}

Hippocampal mossy fiber boutons typically have at least one mitochondrion in the presynaptic bouton (Amaral and Dent, 1981). Schaffer collateral synapses do not always have a mitochondrion in the presynaptic compartment and have been previously described in the CA1 that approximately $40 \%$ of Schaffer collateral boutons have at least one mitochondrion in the presynaptic compartment (Shepherd and Harris, 1998; Smith et al., 2016). I observed that more mossy fiber synaptic profiles contain mitochondria than Schaffer collateral synapses (94.2\% in mossy fiber boutons versus $37.7 \%$ in Schaffer collateral synapses; Figure 26) from cryofixed wild-type slice cultures at DIV28 and could also contribute to the rapid sequestration of calcium in mossy fiber synapses to maintain a low release probability (Kwon et al., 2016). Mitochondria provide local ATP to keep up with demands during high-activity states and are linked with synaptic vesicle mobility during activity (Smith et al., 2016). 
Table 14. Statistical analysis of evoked EPSCs recorded in CA3 and CA1 pyramidal neurons in hippocampal slice cultures.

\begin{tabular}{|c|c|c|c|c|c|c|c|c|c|c|}
\hline & \multicolumn{2}{|c|}{$\begin{array}{l}\text { Paried pulse ratio } \\
\text { (P2/P1; 25 ms } \\
\text { interstimulus } \\
\text { interval) }\end{array}$} & \multicolumn{2}{|c|}{$\begin{array}{l}\text { Paried pulse ratio } \\
\text { (P2/P1; } 50 \text { ms } \\
\text { interstimulus } \\
\text { interval) }\end{array}$} & \multicolumn{2}{|c|}{$\begin{array}{l}\text { Paried pulse ratio } \\
\text { (P2/P1; } 100 \text { ms } \\
\text { interstimulus } \\
\text { interval) }\end{array}$} & \multicolumn{2}{|c|}{$\begin{array}{l}\text { Paried pulse ratio } \\
\text { (P2/P1; } 200 \mathrm{~ms} \\
\text { interstimulus } \\
\text { interval) }\end{array}$} & \multicolumn{2}{|c|}{$\begin{array}{l}\text { Paried pulse ratio } \\
\text { (P2/P1; } 500 \mathrm{~ms} \\
\text { interstimulus } \\
\text { interval) }\end{array}$} \\
\hline $\begin{array}{l}\text { SC } \\
\text { DIV14 }\end{array}$ & $\begin{array}{c}2.203 \\
\pm \\
0.116\end{array}$ & \multirow{4}{*}{$\begin{array}{c}\mathrm{p}=0.96 \mathrm{SC} \\
\text { DIV14 v SC } \\
\text { DIV28 } \\
p=0.24 \mathrm{MF} \\
\text { DIV14 v } \\
\text { MF DIV28 } \\
* * * \\
p<0.001 \\
\text { SC DIV14 v } \\
\text { MF DIV14 } \\
* p=0.04 \\
\text { SC DIV28 v } \\
\text { MF DIV28 }\end{array}$} & $\begin{array}{c}1.960 \\
\pm \\
0.103\end{array}$ & \multirow{4}{*}{$\begin{array}{c}\mathrm{p}=0.87 \mathrm{SC} \\
\text { DIV14 v } \\
\text { SC DIV28 } \\
\mathrm{p}=0.57 \\
\text { MF DIV14 } \\
\text { v MF } \\
\text { DIV28 } \\
\mathrm{p}=0.07 \\
\text { SC DIV14 } \\
\text { v MF } \\
\text { DIV14 } \\
p=0.09 \text { SC } \\
\text { DIV28 v } \\
\text { MF DIV28 }\end{array}$} & $\begin{array}{c}1.630 \\
\pm \\
0.097\end{array}$ & \multirow{4}{*}{$\begin{array}{c}p=0.62 \\
\text { SC DIV14 } \\
\vee \text { SC } \\
\text { DIV28 } \\
p=0.20 \\
\text { MF DIV14 } \\
\vee \text { MF } \\
\text { DIV28 } \\
p=0.17 \\
\text { SC DIV14 } \\
\vee \text { MF } \\
\text { DIV14 } \\
p>0.99 \\
\text { SC DIV28 } \\
v \text { MF } \\
\text { DIV28 }\end{array}$} & $\begin{array}{c}1.468 \\
\pm \\
0.050\end{array}$ & \multirow{4}{*}{$\begin{array}{c}* p=0.03 \\
\text { SC DIV14 } \\
v \text { SC } \\
\text { DIV28 } \\
p=0.60 \\
\text { MF DIV14 } \\
\vee \text { MF } \\
\text { DIV28 } \\
p=0.93 \\
\text { SC DIV14 } \\
v \text { MF } \\
\text { DIV14 } \\
p=0.52 \text { SC } \\
\text { DIV28 v } \\
\text { MF DIV28 }\end{array}$} & $\begin{array}{c}1.198 \\
\pm \\
0.073\end{array}$ & \multirow{4}{*}{$\begin{array}{c}p=0.24 \text { SC } \\
\text { DIV14 v } \\
\text { SC DIV28 } \\
p=0.42 \\
\text { MF DIV14 } \\
\text { v MF } \\
\text { DIV28 } \\
p=0.33 \text { SC } \\
\text { DIV14 v } \\
\text { MF DIV14 } \\
p=0.28 \text { SC } \\
\text { DIV28 v } \\
\text { MF DIV28 }\end{array}$} \\
\hline $\begin{array}{l}\text { SC } \\
\text { DIV28 }\end{array}$ & $\begin{array}{c}2.195 \\
\pm \\
0.160\end{array}$ & & $\begin{array}{c}1.932 \\
\pm \\
0.144\end{array}$ & & $\begin{array}{c}1.696 \\
\pm \\
0.076\end{array}$ & & $\begin{array}{c}1.318 \\
\pm \\
0.078\end{array}$ & & $\begin{array}{c}1.071 \\
\pm \\
0.070\end{array}$ & \\
\hline $\begin{array}{l}\text { MF } \\
\text { DIV14 }\end{array}$ & $\begin{array}{c}2.981 \\
\pm \\
0.145\end{array}$ & & $\begin{array}{c}2.218 \\
\pm \\
0.082\end{array}$ & & $\begin{array}{c}1.851 \\
\pm \\
0.093\end{array}$ & & $\begin{array}{c}1.456 \\
\pm \\
0.088\end{array}$ & & $\begin{array}{c}1.096 \\
\pm \\
0.071\end{array}$ & \\
\hline $\begin{array}{l}\text { MF } \\
\text { DIV28 }\end{array}$ & $\begin{array}{c}2.712 \\
\pm \\
0.169\end{array}$ & & $\begin{array}{c}2.317 \\
\pm \\
0.157\end{array}$ & & $\begin{array}{c}1.754 \\
\pm \\
0.123\end{array}$ & & $\begin{array}{c}1.391 \\
\pm \\
0.081\end{array}$ & & $\begin{array}{c}1.177 \\
\pm \\
0.067\end{array}$ & \\
\hline
\end{tabular}


Table 15. Statistical analysis of vesicle distributions in Schaffer collateral and mossy fiberCA3 synapses in organotypic slice cultures high-pressure frozen at DIV14.

\begin{tabular}{|c|c|c|c|c|c|c|c|c|}
\hline & \multicolumn{2}{|c|}{$\begin{array}{l}\text { Docked vesicles }(0-2 \mathrm{~nm}) \\
\text { per } 0.01 \mu \mathrm{m}^{2} \mathrm{AZ} \text { area }\end{array}$} & \multicolumn{2}{|c|}{$\begin{array}{l}\text { Vesicles }(0-20 \mathrm{~nm}) \text { per } \\
0.01 \mu \mathrm{m}^{2} \mathrm{AZ} \text { area }\end{array}$} & \multicolumn{2}{|c|}{$\begin{array}{l}\text { Vesicles }(0-40 \mathrm{~nm}) \text { per } \\
0.01 \mu \mathrm{m}^{2} \mathrm{AZ} \text { area }\end{array}$} & \multicolumn{2}{|c|}{$\begin{array}{l}\text { Vesicles }(0-100 \mathrm{~nm}) \text { per } \\
0.01 \mu \mathrm{m}^{2} \mathrm{AZ} \text { area }\end{array}$} \\
\hline \multicolumn{9}{|c|}{ Synaptic vesicles } \\
\hline SC & $1.015 \pm$ & \multirow{3}{*}{$* * * p<0.001$} & $1.63 \pm$ & \multirow{3}{*}{$* p=0.01$} & $2.137 \pm$ & \multirow{3}{*}{$* p=0.04$} & $6.534 \pm$ & \multirow{3}{*}{$p=0.15$} \\
\hline DIV14 & 0.112 & & 0.140 & & 0.137 & & 0.276 & \\
\hline $\begin{array}{l}\text { MF } \\
\text { DIV14 }\end{array}$ & $\begin{array}{c}0.458 \pm \\
0.084 \\
\end{array}$ & & $\begin{array}{c}1.117 \pm \\
0.654 \\
\end{array}$ & & $\begin{array}{c}1.718 \pm \\
0.149 \\
\end{array}$ & & $\begin{array}{c}5.811 \pm \\
0.425 \\
\end{array}$ & \\
\hline \multicolumn{9}{|c|}{ Synaptic vesicles and giant vesicles } \\
\hline $\begin{array}{l}\text { SC } \\
\text { DIV14 }\end{array}$ & $1.015 \pm$ & \multirow{3}{*}{$* * \mathrm{p}=0.003$} & $\begin{array}{l}1.63 \pm \\
0.140\end{array}$ & \multirow{3}{*}{$p=0.30$} & $\begin{array}{c}2.137 \pm \\
0.137\end{array}$ & \multirow{3}{*}{$p=0.74$} & $6.534 \pm$ & \multirow{3}{*}{$p=0.71$} \\
\hline $\mathrm{MF}$ & $0.576 \pm$ & & $1.413 \pm$ & & $2.072 \pm$ & & $6.356 \pm$ & \\
\hline DIV14 & 0.087 & & 0.121 & & 0.141 & & 0.415 & \\
\hline \multicolumn{9}{|c|}{ Giant vesicles } \\
\hline $\begin{array}{l}\text { SC } \\
\text { DIV14 }\end{array}$ & - & & - & & - & & $\begin{array}{c}0.028 \pm \\
0.016\end{array}$ & \multirow{3}{*}{$* * * p<0.001$} \\
\hline MF & $0.118 \pm$ & & $0.296 \pm$ & & $0.355 \pm$ & & $0.545 \pm$ & \\
\hline DIV14 & 0.034 & & 0.052 & & 0.055 & & 0.083 & \\
\hline \multicolumn{9}{|c|}{ Dense-core vesicles } \\
\hline $\begin{array}{l}\text { SC } \\
\text { DIV14 }\end{array}$ & - & & - & & - & & - & \\
\hline MF & $0.075 \pm$ & & $0.161 \pm$ & & $0.163 \pm$ & & $0.278 \pm$ & \\
\hline DIV14 & 0.023 & & 0.048 & & 0.036 & & 0.049 & \\
\hline
\end{tabular}


Table 16. Statistical analysis of vesicle diameters in Schaffer collateral and mossy fiber-CA3 synapses in organotypic slice cultures high-pressure frozen at DIV14.

\begin{tabular}{|c|c|c|c|c|c|c|c|c|}
\hline & \multicolumn{2}{|c|}{$\begin{array}{l}\text { Non-docked vesicle } \\
\text { diameter }(\mathrm{nm})\end{array}$} & \multicolumn{2}{|c|}{$\begin{array}{l}\text { Docked vesicle diameter } \\
(\mathrm{nm})\end{array}$} & \multicolumn{2}{|c|}{$\begin{array}{l}\text { Proportion of docked } \\
\text { vesicles (\%) }\end{array}$} & \multicolumn{2}{|c|}{$A Z$ area $\left(0.01 \mu \mathrm{m}^{2}\right)$} \\
\hline \multicolumn{9}{|c|}{ Synaptic vesicles } \\
\hline SC DIV14 & $\begin{array}{l}43.43 \pm \\
0.258\end{array}$ & \multirow{2}{*}{$* * p=0.003$} & $\begin{array}{c}43.32 \pm \\
0.354 \\
\text { ( } n=116 \\
\text { vesicles) }\end{array}$ & \multirow{2}{*}{$\begin{array}{c}* * * \\
\mathrm{p}<0.001\end{array}$} & $100 \pm 0$ & \multirow{2}{*}{$\begin{array}{c}* * * \\
\mathrm{p}<0.001\end{array}$} & $\begin{array}{l}3.495 \pm \\
0.185\end{array}$ & \multirow{2}{*}{$* * p=0.003$} \\
\hline MF DIV14 & $\begin{array}{c}44.69 \pm \\
0.311\end{array}$ & & $\begin{array}{c}46.78 \pm \\
0.460 \\
(n=66 \\
\text { vesicles) }\end{array}$ & & $\begin{array}{c}77.53 \pm \\
5.635\end{array}$ & & $\begin{array}{c}4.470 \pm \\
0.260\end{array}$ & \\
\hline \multicolumn{5}{|c|}{ Synaptic vesicles and giant vesicles } & \multicolumn{2}{|c|}{$\begin{array}{l}\text { Vesicles } 0-2 \mathrm{~nm} \text { per } \\
\text { vesicles } 0-40 \mathrm{~nm}\end{array}$} & & \\
\hline SC DIV14 & $\begin{array}{l}43.43 \pm \\
0.258\end{array}$ & \multirow{2}{*}{$\begin{array}{c}* * * \\
\mathrm{p}<0.001\end{array}$} & $\begin{array}{c}43.32 \pm \\
0.354 \\
\text { ( } n=116 \\
\text { vesicles) }\end{array}$ & \multirow{2}{*}{$\begin{array}{c}* * * \\
\mathrm{p}<0.001\end{array}$} & $\begin{array}{c}0.471 \pm \\
0.040\end{array}$ & \multirow{2}{*}{$* * p=0.002$} & & \\
\hline MF DIV14 & $\begin{array}{c}48.52 \pm \\
0.719\end{array}$ & & $\begin{array}{c}53.96 \pm \\
1.859 \\
\text { ( } n=81 \\
\text { vesicles) }\end{array}$ & & $\begin{array}{c}0.297 \pm \\
0.038\end{array}$ & & & \\
\hline \multicolumn{7}{|c|}{ Giant vesicles } & & \\
\hline SC DIV14 & - & & $\begin{array}{c}69.62 \pm \\
4.231 \\
\text { (3 vesicles) }\end{array}$ & & $0 \pm 0$ & \multirow{2}{*}{$\begin{array}{c}* * * \\
p<0.001\end{array}$} & & \\
\hline MF DIV14 & $\begin{array}{c}84.86 \pm \\
1.450 \\
(n=83 \\
\text { vesicles) }\end{array}$ & & $\begin{array}{c}85.55 \pm \\
3.720 \\
(n=15 \\
\text { vesicles) }\end{array}$ & & $\begin{array}{c}22.47 \pm \\
5.635\end{array}$ & & & \\
\hline \multicolumn{7}{|c|}{ Dense-core vesicles } & & \\
\hline SC DIV14 & - & & - & & & & & \\
\hline MF DIV14 & $\begin{array}{c}73.406 \pm \\
1.820 \\
\text { ( } n=27 \\
\text { vesicles) }\end{array}$ & & $\begin{array}{c}68.44 \pm \\
4.832 \\
\text { ( } n=10 \\
\text { vesicles) }\end{array}$ & & & & & \\
\hline $\begin{array}{l}\text { Abbreviatic } \\
\text { data; } p \text {-val }\end{array}$ & $\begin{array}{l}\text { s: AZ, Activ } \\
\text { s in blue ar }\end{array}$ & $\begin{array}{l}\text { ne; SC, Sch } \\
\text { m non-nc }\end{array}$ & $\begin{array}{l}\text { r collatera } \\
\text { Illy distribu }\end{array}$ & $\begin{array}{l}\text { mossy } \\
\text { tata }\end{array}$ & P-value & reen are & normal & istributed \\
\hline
\end{tabular}


Table 17. Statistical analysis of vesicle distributions in Schaffer collateral and mossy fiberCA3 synapses from organotypic slice cultures high-pressure frozen at DIV28.

\begin{tabular}{|c|c|c|c|c|c|c|c|c|}
\hline & \multicolumn{2}{|c|}{$\begin{array}{l}\text { Docked vesicles } \\
(0-2 \mathrm{~nm}) \text { per } 0.01 \mu \mathrm{m}^{2} \\
\text { AZ area }\end{array}$} & \multicolumn{2}{|c|}{$\begin{array}{l}\text { Vesicles }(0-20 \mathrm{~nm}) \text { per } \\
0.01 \mu \mathrm{m}^{2} \mathrm{AZ} \text { area }\end{array}$} & \multicolumn{2}{|c|}{$\begin{array}{l}\text { Vesicles }(0-40 \mathrm{~nm}) \text { per } \\
0.01 \mu \mathrm{m}^{2} \mathrm{AZ} \text { area }\end{array}$} & \multicolumn{2}{|c|}{$\begin{array}{l}\text { Vesicles }(0-100 \mathrm{~nm}) \text { per } \\
0.01 \mu \mathrm{m}^{2} \mathrm{AZ} \text { area }\end{array}$} \\
\hline \multicolumn{9}{|c|}{ Synaptic vesicles } \\
\hline SC DIV28 & $\begin{array}{c}1.710 \pm \\
0.139\end{array}$ & \multirow{2}{*}{$\begin{array}{c}* * * \\
\mathrm{p}<0.001\end{array}$} & $\begin{array}{c}1.830 \pm \\
0.144\end{array}$ & \multirow{2}{*}{$p=0.06$} & $\begin{array}{c}2.312 \pm \\
0.148\end{array}$ & \multirow{2}{*}{$p=0.32$} & $\begin{array}{c}6.462 \pm \\
0.348\end{array}$ & \multirow{2}{*}{$p=0.27$} \\
\hline MF DIV28 & $\begin{array}{c}0.967 \pm \\
0.132\end{array}$ & & $\begin{array}{c}1.531 \pm \\
0.123\end{array}$ & & $\begin{array}{c}2.094 \pm \\
0.159\end{array}$ & & $\begin{array}{c}5.815 \pm \\
0.469\end{array}$ & \\
\hline \multicolumn{9}{|c|}{ Synaptic vesicles and giant vesicles } \\
\hline SC DIV28 & $\begin{array}{c}1.710 \pm \\
0.139 \\
\end{array}$ & \multirow{2}{*}{$\begin{array}{c}* * * \\
\mathrm{p}<0.001\end{array}$} & $\begin{array}{c}1.830 \pm \\
0.144 \\
\end{array}$ & \multirow{2}{*}{$p=0.47$} & $\begin{array}{c}2.312 \pm \\
0.148 \\
\end{array}$ & \multirow{2}{*}{$p=0.95$} & $\begin{array}{c}6.478 \pm \\
0.347 \\
\end{array}$ & \multirow{2}{*}{$p=0.63$} \\
\hline MF DIV28 & $\begin{array}{c}1.061 \pm \\
0.141\end{array}$ & & $\begin{array}{c}1.752 \pm \\
0.129\end{array}$ & & $\begin{array}{c}2.300 \pm \\
0.164\end{array}$ & & $\begin{array}{c}6.193 \pm \\
0.470\end{array}$ & \\
\hline \multicolumn{9}{|c|}{ Giant vesicles } \\
\hline SC DIV28 & - & & - & & - & & $\begin{array}{c}0.015 \pm \\
0.011\end{array}$ & $* * *$ \\
\hline MF DIV28 & $\begin{array}{c}0.093 \pm \\
0.028 \\
\end{array}$ & & $\begin{array}{c}0.222 \pm \\
0.054\end{array}$ & & $\begin{array}{c}0.248 \pm \\
0.055\end{array}$ & & $\begin{array}{c}0.378 \pm \\
0.063 \\
\end{array}$ & $p<0.001$ \\
\hline \multicolumn{9}{|c|}{ Dense-core vesicles } \\
\hline SC DIV28 & - & & - & & - & & - & \\
\hline MF DIV28 & $\begin{array}{l}0.05 \pm \\
0.019\end{array}$ & & $\begin{array}{c}0.174 \pm \\
0.031\end{array}$ & & $\begin{array}{c}0.190 \pm \\
0.032\end{array}$ & & $\begin{array}{c}0.282 \pm \\
0.047\end{array}$ & \\
\hline
\end{tabular}


Table 18. Statistical analysis of vesicle diameters in Schaffer collateral and mossy fiber-CA3 synapses in organotypic slice cultures high-pressure frozen at DIV28.

\begin{tabular}{|c|c|c|c|c|c|c|c|c|}
\hline & \multicolumn{2}{|c|}{$\begin{array}{l}\text { Non-docked vesicle } \\
\text { diameter }(\mathrm{nm})\end{array}$} & \multicolumn{2}{|c|}{$\begin{array}{l}\text { Docked vesicle diameter } \\
\text { (nm) }\end{array}$} & \multicolumn{2}{|c|}{$\begin{array}{l}\text { Proportion of docked } \\
\text { vesicles (\%) }\end{array}$} & \multicolumn{2}{|c|}{$A Z$ area $\left(\mu \mathrm{m}^{2}\right)$} \\
\hline \multicolumn{9}{|c|}{ Synaptic vesicles } \\
\hline SC DIV28 & $\begin{array}{c}44.13 \pm \\
0.175\end{array}$ & \multirow{2}{*}{$* p=0.02$} & $\begin{array}{c}44.37 \pm \\
0.230(222 \\
\text { vesicles) }\end{array}$ & \multirow{2}{*}{$\begin{array}{c}* * * \\
p<0.001\end{array}$} & $100 \pm 0$ & \multirow{2}{*}{$\begin{array}{c}* * * \\
p<0.001\end{array}$} & $\begin{array}{c}4.558 \pm \\
0.279\end{array}$ & \multirow{2}{*}{$p=0.08$} \\
\hline MF DIV28 & $\begin{array}{l}45.33 \pm \\
0.443\end{array}$ & & $\begin{array}{c}45.77 \pm \\
0.373 \text { (144 } \\
\text { vesicles) }\end{array}$ & & $\begin{array}{c}88.33 \pm \\
4.251\end{array}$ & & $\begin{array}{c}5.533 \pm \\
0.396\end{array}$ & \\
\hline \multicolumn{5}{|c|}{ Synaptic vesicles and giant vesicles } & \multicolumn{2}{|c|}{$\begin{array}{l}\text { Vesicles } 0-2 \mathrm{~nm} \text { per } \\
\text { vesicles } 0-40 \mathrm{~nm}\end{array}$} & & \\
\hline SC DIV28 & $\begin{array}{c}44.20 \pm \\
0.188\end{array}$ & \multirow{2}{*}{$\begin{array}{c}* * * \\
\mathrm{p}<0.001\end{array}$} & $\begin{array}{c}44.37 \pm \\
0.230(222 \\
\text { vesicles) }\end{array}$ & \multirow{2}{*}{$\begin{array}{c}* * * \\
\mathrm{p}<0.001\end{array}$} & $\begin{array}{c}0.724 \pm \\
0.032\end{array}$ & \multirow{2}{*}{$\begin{array}{c}* * * \\
p<0.001\end{array}$} & & \\
\hline MF DIV28 & $\begin{array}{l}48.44 \pm \\
1.122\end{array}$ & & $\begin{array}{c}49.40 \pm \\
1.034 \text { (159 } \\
\text { vesicles) }\end{array}$ & & $\begin{array}{c}0.452 \pm \\
0.042\end{array}$ & & & \\
\hline \multicolumn{7}{|c|}{ Giant vesicles } & & \\
\hline SC DIV28 & $\begin{array}{l}66.51 \pm \\
5.284(2 \\
\text { vesicles) }\end{array}$ & & - & & $0 \pm 0$ & \multirow{2}{*}{$\begin{array}{c}* * * \\
\mathrm{p}<0.001\end{array}$} & & \\
\hline MF DIV28 & $\begin{array}{c}83.41 \pm \\
2.205 \text { (44 } \\
\text { vesicles) }\end{array}$ & & $\begin{array}{c}84.27 \pm \\
4.292(15 \\
\text { vesicles) }\end{array}$ & & $\begin{array}{c}11.67 \pm \\
4.251\end{array}$ & & & \\
\hline \multicolumn{7}{|c|}{ Dense-core vesicles } & & \\
\hline SC DIV28 & - & & - & & & & & \\
\hline MF DIV28 & $\begin{array}{c}72.85 \pm \\
2.379 \text { (39 } \\
\text { vesicles) }\end{array}$ & & $\begin{array}{c}78.01 \pm \\
3.001 \text { (8 } \\
\text { vesicles) }\end{array}$ & & & & & \\
\hline
\end{tabular}


Table 19. Statistical analysis of vesicle distributions in Schaffer collateral and mossy fiberCA3 synapses in wild-type acute slice preparations high-pressure frozen at P18.

\begin{tabular}{|c|c|c|c|c|c|c|c|c|}
\hline & \multicolumn{2}{|c|}{$\begin{array}{l}\text { Docked vesicles } \\
(0-2 \mathrm{~nm}) \text { per } 0.01 \mathrm{\mu m}^{2} \\
\text { AZ area }\end{array}$} & \multicolumn{2}{|c|}{$\begin{array}{l}\text { Vesicles }(0-20 \mathrm{~nm}) \text { per } \\
0.01 \mu \mathrm{m}^{2} \mathrm{AZ} \text { area }\end{array}$} & \multicolumn{2}{|c|}{$\begin{array}{l}\text { Vesicles }(0-40 \mathrm{~nm}) \text { per } \\
0.01 \mu^{2} \mathrm{AZ} \text { area }\end{array}$} & \multicolumn{2}{|c|}{$\begin{array}{l}\text { Vesicles }(0-100 \mathrm{~nm}) \text { per } \\
0.01 \mathrm{\mu m}^{2} \mathrm{AZ} \text { area }\end{array}$} \\
\hline \multicolumn{9}{|c|}{ Synaptic vesicles } \\
\hline $\begin{array}{l}\text { SC P18 } \\
\text { Acute }\end{array}$ & $\begin{array}{c}1.583 \pm \\
0.166 \\
\end{array}$ & \multirow{2}{*}{$* * p=0.004$} & $\begin{array}{c}2.177 \pm \\
0.176 \\
\end{array}$ & \multirow{2}{*}{$p=0.05$} & $\begin{array}{c}2.659 \pm \\
0.213\end{array}$ & \multirow{2}{*}{$p=0.48$} & $\begin{array}{c}6.848 \pm \\
0.437 \\
\end{array}$ & \multirow{2}{*}{$p=0.47$} \\
\hline $\begin{array}{l}\text { MF P18 } \\
\text { Acute }\end{array}$ & $\begin{array}{c}1.059 \pm \\
0.088\end{array}$ & & $\begin{array}{c}1.751 \pm \\
0.128\end{array}$ & & $\begin{array}{c}2.454 \pm \\
0.185\end{array}$ & & $\begin{array}{c}6.639 \pm \\
0.476\end{array}$ & \\
\hline \multicolumn{9}{|c|}{ Synaptic vesicles and giant vesicles } \\
\hline $\begin{array}{l}\text { SC P18 } \\
\text { Acute }\end{array}$ & $\begin{array}{c}1.608 \pm \\
0.163 \\
\end{array}$ & \multirow{2}{*}{$* * p=0.009$} & $\begin{array}{c}2.190 \pm \\
0.174 \\
\end{array}$ & \multirow{2}{*}{$p=0.17$} & $\begin{array}{c}2.671 \pm \\
0.211 \\
\end{array}$ & \multirow{2}{*}{$p=0.72$} & $\begin{array}{c}6.873 \pm \\
0.437 \\
\end{array}$ & \multirow{2}{*}{$p=0.66$} \\
\hline $\begin{array}{l}\text { MF P18 } \\
\text { Acute }\end{array}$ & $\begin{array}{l}1.128 \pm \\
0.095\end{array}$ & & $\begin{array}{c}1.857 \pm \\
0.131 \\
\end{array}$ & & $\begin{array}{c}2.566 \pm \\
0.188\end{array}$ & & $\begin{array}{l}6.82 \pm \\
0.488 \\
\end{array}$ & \\
\hline \multicolumn{9}{|c|}{ Giant vesicles } \\
\hline $\begin{array}{l}\text { SC P18 } \\
\text { Acute }\end{array}$ & $\begin{array}{c}0.012 \pm \\
0.012\end{array}$ & \multirow{2}{*}{$* \mathrm{p}=0.02$} & $\begin{array}{c}0.012 \pm \\
0.012 \\
\end{array}$ & \multirow{2}{*}{$* * p=0.002$} & $\begin{array}{c}0.012 \pm \\
0.012 \\
\end{array}$ & \multirow{2}{*}{$* * p=0.001$} & $\begin{array}{c}0.012 \pm \\
0.012 \\
\end{array}$ & \multirow{2}{*}{$\begin{array}{c}* * * \\
\mathrm{p}<0.001\end{array}$} \\
\hline $\begin{array}{l}\text { MF P18 } \\
\text { Acute }\end{array}$ & $\begin{array}{c}0.069 \pm \\
0.020\end{array}$ & & $\begin{array}{c}0.106 \pm \\
0.025 \\
\end{array}$ & & $\begin{array}{c}0.112 \pm \\
0.024\end{array}$ & & $\begin{array}{c}0.185 \pm \\
0.037\end{array}$ & \\
\hline \multicolumn{9}{|c|}{ Dense-core vesicles } \\
\hline $\begin{array}{l}\text { SC P18 } \\
\text { Acute }\end{array}$ & - & & - & & $\begin{array}{c}0.013 \pm \\
0.013 \\
\end{array}$ & \multirow{2}{*}{$\begin{array}{c}* * * \\
\mathrm{p}<0.001\end{array}$} & $\begin{array}{c}0.048 \pm \\
0.029 \\
\end{array}$ & \multirow{2}{*}{$\begin{array}{c}* * * \\
\mathrm{p}<0.001\end{array}$} \\
\hline $\begin{array}{l}\text { MF P18 } \\
\text { Acute }\end{array}$ & $\begin{array}{c}0.072 \pm \\
0.021\end{array}$ & & $\begin{array}{c}0.134 \pm \\
0.033\end{array}$ & & $\begin{array}{c}0.209 \pm \\
0.034\end{array}$ & & $\begin{array}{c}0.411 \pm \\
0.065\end{array}$ & \\
\hline
\end{tabular}


Table 20. Statistical analysis of vesicle diameters in Schaffer collateral and mossy fiber-CA3 synapses from wild-type acute slice preparations high-pressure frozen at P18.

\begin{tabular}{|c|c|c|c|c|c|c|c|c|}
\hline & \multicolumn{2}{|c|}{$\begin{array}{l}\text { Non-docked vesicle } \\
\text { diameter }(\mathrm{nm})\end{array}$} & \multicolumn{2}{|c|}{$\begin{array}{l}\text { Docked vesicle } \\
\text { diameter }(\mathrm{nm})\end{array}$} & \multicolumn{2}{|c|}{$\begin{array}{l}\text { Proportion of docked } \\
\text { vesicles (\%) }\end{array}$} & \multicolumn{2}{|c|}{ AZ area $\left(0.01 \mu \mathrm{m}^{2}\right)$} \\
\hline \multicolumn{9}{|c|}{ Synaptic vesicles } \\
\hline $\begin{array}{l}\text { SC P18 } \\
\text { Acute }\end{array}$ & $\begin{array}{l}45.27 \pm \\
0.428\end{array}$ & \multirow{2}{*}{$\begin{array}{c}* * * \\
\mathrm{p}<0.001\end{array}$} & $\begin{array}{c}45.46 \pm \\
0.292 \\
(n=112 \\
\text { vesicles) }\end{array}$ & \multirow{2}{*}{$\begin{array}{c}* * * \\
p<0.001\end{array}$} & $98.95 \pm 1.053$ & \multirow{2}{*}{$* p=0.02$} & $\begin{array}{c}3.979 \pm \\
0.226\end{array}$ & $* *$ \\
\hline $\begin{array}{l}\text { MF P18 } \\
\text { Acute }\end{array}$ & $\begin{array}{c}46.82 \pm \\
0.208\end{array}$ & & $\begin{array}{c}47.93 \pm \\
0.233 \\
(n=185 \\
\text { vesicles) }\end{array}$ & & $\begin{array}{c}94.63 \pm 1.595 \\
(29 \\
\text { tomograms })\end{array}$ & & $\begin{array}{c}5.538 \pm \\
0.331\end{array}$ & $p=0.001$ \\
\hline \multicolumn{5}{|c|}{ Synaptic vesicles and giant vesicles } & \multicolumn{2}{|c|}{$\begin{array}{l}\text { Vesicles } 0-2 \mathrm{~nm} \text { per } \\
\text { vesicles } 0-40 \mathrm{~nm}\end{array}$} & & \\
\hline $\begin{array}{l}\text { SC P18 } \\
\text { Acute }\end{array}$ & $\begin{array}{c}45.27 \pm \\
0.428\end{array}$ & \multirow{2}{*}{$\begin{array}{c}* * * \\
\mathrm{p}<0.001\end{array}$} & $\begin{array}{c}45.59 \pm \\
0.318 \\
\text { ( } n=113 \\
\text { vesicles) }\end{array}$ & \multirow{2}{*}{$\begin{array}{c}* * * \\
\mathrm{p}<0.001\end{array}$} & $0.617 \pm 0.053$ & \multirow{2}{*}{$\begin{array}{c}* * \\
p=0.009\end{array}$} & & \\
\hline $\begin{array}{l}\text { MF P18 } \\
\text { Acute }\end{array}$ & $\begin{array}{l}47.66 \pm \\
0.350\end{array}$ & & $\begin{array}{c}50.67 \pm \\
0.842 \\
(n=197 \\
\text { vesicles) }\end{array}$ & & $0.448 \pm 0.037$ & & & \\
\hline \multicolumn{7}{|c|}{ Giant vesicles } & & \\
\hline $\begin{array}{l}\text { SC P18 } \\
\text { Acute }\end{array}$ & - & & $\begin{array}{c}60.3 \\
(n=1 \\
\text { vesicle) }\end{array}$ & & $1.053 \pm 1.053$ & \multirow[b]{2}{*}{$* p=0.02$} & & \\
\hline $\begin{array}{l}\text { MF P18 } \\
\text { Acute }\end{array}$ & $\begin{array}{c}77.86 \pm \\
4.901 \\
(n=17 \\
\text { vesicles) }\end{array}$ & & $\begin{array}{c}92.85 \pm \\
4.585 \\
(n=12 \\
\text { vesicles) }\end{array}$ & & $\begin{array}{c}5.366 \pm 1.595 \\
(29 \\
\text { tomograms })\end{array}$ & & & \\
\hline \multicolumn{7}{|c|}{ Dense-core vesicles } & & \\
\hline $\begin{array}{l}\text { SC P18 } \\
\text { Acute }\end{array}$ & $\begin{array}{c}70.74 \pm \\
4.054 \\
(n=5 \\
\text { vesicles) }\end{array}$ & & - & & & & & \\
\hline $\begin{array}{l}\text { MF P18 } \\
\text { Acute }\end{array}$ & $\begin{array}{c}65.52 \pm \\
1.994 \\
(n=50 \\
\text { vesicles) }\end{array}$ & & $\begin{array}{c}75.46 \pm \\
4.326 \\
\text { ( } n=10 \\
\text { vesicles) }\end{array}$ & & & & & \\
\hline
\end{tabular}


Table 21. Statistical analysis of vesicle distributions in mossy fiber synapses from wild-type mice perfusion-fixed at P28.

\begin{tabular}{|c|c|c|c|c|c|c|c|c|}
\hline & \multicolumn{2}{|c|}{$\begin{array}{l}\text { Docked vesicles (0- } \\
2 \mathrm{~nm} \text { ) per } 0.01 \mu \mathrm{m}^{2} \mathrm{AZ} \\
\text { area }\end{array}$} & \multicolumn{2}{|c|}{$\begin{array}{l}\text { Vesicles }(0-20 \mathrm{~nm}) \text { per } \\
0.01 \mu \mathrm{m}^{2} \mathrm{AZ} \text { area }\end{array}$} & \multicolumn{2}{|c|}{$\begin{array}{l}\text { Vesicles }(0-40 \mathrm{~nm}) \text { per } \\
0.01 \mu \mathrm{m}^{2} \mathrm{AZ} \text { area }\end{array}$} & \multicolumn{2}{|c|}{$\begin{array}{l}\text { Vesicles }(0-100 \mathrm{~nm}) \text { per } \\
0.01 \mu \mathrm{m}^{2} \mathrm{AZ} \text { area }\end{array}$} \\
\hline \multicolumn{9}{|c|}{ Synaptic vesicles } \\
\hline $\begin{array}{l}\text { MF PF1 } \\
\text { P28 }\end{array}$ & $\begin{array}{c}0.027 \pm \\
0.019\end{array}$ & \multirow{2}{*}{$* * p=0.002$} & $\begin{array}{c}0.470 \pm \\
0.097\end{array}$ & \multirow{2}{*}{$* * p=0.003$} & $\begin{array}{c}1.508 \pm \\
0.152\end{array}$ & \multirow{2}{*}{$\begin{array}{c}* * * \\
\mathrm{p}<0.001\end{array}$} & $\begin{array}{c}4.683 \pm \\
0.655\end{array}$ & \multirow{2}{*}{$\begin{array}{c}* * * \\
\mathrm{p}<0.001\end{array}$} \\
\hline $\begin{array}{l}\text { MF PF2 } \\
\text { P28 }\end{array}$ & $\begin{array}{c}0.381 \pm \\
0.106\end{array}$ & & $\begin{array}{c}1.018 \pm \\
0.132\end{array}$ & & $\begin{array}{c}2.809 \pm \\
0.202\end{array}$ & & $\begin{array}{c}9.415 \pm \\
0.754\end{array}$ & \\
\hline \multicolumn{9}{|c|}{ Synaptic vesicles and giant vesicles } \\
\hline $\begin{array}{l}\text { MF PF1 } \\
\text { P28 }\end{array}$ & $\begin{array}{c}0.027 \pm \\
0.019 \\
\end{array}$ & \multirow{2}{*}{$* * p=0.002$} & $\begin{array}{c}0.493 \pm \\
0.094\end{array}$ & \multirow{2}{*}{$\begin{array}{c}* * * \\
p<0.001\end{array}$} & $\begin{array}{c}1.573 \pm \\
0.150 \\
\end{array}$ & \multirow{2}{*}{$\begin{array}{c}* * * \\
\mathrm{p}<0.001\end{array}$} & $\begin{array}{c}4.887 \pm \\
0.651 \\
\end{array}$ & \multirow{2}{*}{$\begin{array}{c}* * * \\
\mathrm{p}<0.001\end{array}$} \\
\hline $\begin{array}{l}\text { MF PF2 } \\
\text { P28 }\end{array}$ & $\begin{array}{c}0.394 \pm \\
0.107\end{array}$ & & $\begin{array}{c}1.127 \pm \\
0.120\end{array}$ & & $\begin{array}{l}2.954 \pm \\
0.195\end{array}$ & & $\begin{array}{c}9.678 \pm \\
0.754\end{array}$ & \\
\hline \multicolumn{9}{|c|}{ Giant vesicles } \\
\hline $\begin{array}{l}\text { MF PF1 } \\
\text { P28 }\end{array}$ & $0 \pm 0$ & \multirow{2}{*}{$p>0.99$} & $\begin{array}{c}0.023 \pm \\
0.016\end{array}$ & \multirow{2}{*}{$p=0.11$} & $\begin{array}{c}0.065 \pm \\
0.035\end{array}$ & \multirow{2}{*}{$p=0.45$} & $\begin{array}{c}0.205 \pm \\
0.073\end{array}$ & \multirow{2}{*}{$p=0.69$} \\
\hline $\begin{array}{l}\text { MF PF2 } \\
\text { P28 }\end{array}$ & $\begin{array}{c}0.013 \pm \\
0.013\end{array}$ & & $\begin{array}{c}0.109 \pm \\
0.040\end{array}$ & & $\begin{array}{c}0.145 \pm \\
0.058\end{array}$ & & $\begin{array}{c}0.263 \pm \\
0.076\end{array}$ & \\
\hline \multicolumn{9}{|c|}{ Dense-core vesicles } \\
\hline $\begin{array}{l}\text { MF PF1 } \\
\text { P28 }\end{array}$ & $0 \pm 0$ & \multirow{2}{*}{$* * p=0.01$} & $\begin{array}{c}0.084 \pm \\
0.038 \\
\end{array}$ & \multirow{2}{*}{$p=0.22$} & $\begin{array}{c}0.195 \pm \\
0.049 \\
\end{array}$ & \multirow{2}{*}{$p=0.62$} & $\begin{array}{c}0.241 \pm \\
0.049 \\
\end{array}$ & \multirow{2}{*}{$p=0.94$} \\
\hline $\begin{array}{l}\text { MF PF2 } \\
\text { P28 }\end{array}$ & $\begin{array}{c}0.141 \pm \\
0.043\end{array}$ & & $\begin{array}{c}0.184 \pm \\
0.057\end{array}$ & & $\begin{array}{c}0.184 \pm \\
0.057\end{array}$ & & $\begin{array}{c}0.255 \pm \\
0.057\end{array}$ & \\
\hline \multicolumn{9}{|c|}{$\begin{array}{l}\text { Abbreviations: AZ, Active zone; MF, mossy fiber; PF1, perfusion fixation protocol } 1 \text { (ice-cold } 4 \% \text { PFA, } 2.5 \% \text { GA in } 0.1 \mathrm{M} \mathrm{PB} \text {, } \\
\text { pH 7.4) (Rollenhagen et al., 2007); PF2, perfusion fixation protocol } 2\left(37^{\circ} \mathrm{C} 2 \% \text { PFA, } 2.5 \% \mathrm{GA}, 2 \mathrm{mM} \mathrm{CaCl} 2 \text { in } 0.1 \mathrm{M}\right. \\
\text { cacodylate buffer, pH 7.4) (Chicurel and Harris, 1992). P-values in green are from normally distributed data; } p \text {-values in } \\
\text { blue are from non-normally distributed data. P28 wild-type mice were perfusion-fixed in this study. }\end{array}$} \\
\hline
\end{tabular}


Table 22. Statistical analysis of vesicle diameters in mossy fiber synapses from wild-type mice perfusion-fixed at P28.

\begin{tabular}{|c|c|c|c|c|c|c|c|}
\hline & \multicolumn{2}{|c|}{$\begin{array}{l}\text { Non-docked vesicle } \\
\text { diameter }(\mathrm{nm})\end{array}$} & $\begin{array}{l}\text { Docked vesicle } \\
\text { diameter }(\mathrm{nm})\end{array}$ & \multicolumn{2}{|c|}{$\begin{array}{l}\text { Proportion of docked } \\
\text { vesicles (\%) }\end{array}$} & \multicolumn{2}{|c|}{ AZ area $\left(0.01 \mu \mathrm{m}^{2}\right)$} \\
\hline \multicolumn{8}{|c|}{ Synaptic vesicles } \\
\hline $\begin{array}{l}\text { MF PF1 } \\
\text { P28 }\end{array}$ & $\begin{array}{l}45.77 \pm \\
0.335\end{array}$ & \multirow{2}{*}{$p=0.49$} & $\begin{array}{c}36.67 \pm \\
5.284 \\
(n=2 \\
\text { vesicles) }\end{array}$ & $\begin{array}{c}100 \pm 0(2 \\
\text { tomograms) }\end{array}$ & & $\begin{array}{c}5.122 \pm \\
0.592\end{array}$ & \multirow{2}{*}{$p=0.06$} \\
\hline $\begin{array}{l}\text { MF PF2 } \\
\text { P28 }\end{array}$ & $\begin{array}{c}45.44 \pm \\
0.323\end{array}$ & & $\begin{array}{c}44.93 \pm \\
1.341 \\
\text { ( } n=21 \\
\text { vesicles) }\end{array}$ & $\begin{array}{c}83.33 \pm 9.796 \\
(11 \\
\text { tomograms })\end{array}$ & & $\begin{array}{c}3.854 \pm \\
0.307\end{array}$ & \\
\hline \multicolumn{4}{|c|}{ Synaptic vesicles and giant vesicles } & \multicolumn{2}{|c|}{$\begin{array}{l}\text { Vesicles } 0-2 \mathrm{~nm} \text { per vesicles } \\
0-40 \mathrm{~nm}\end{array}$} & & \\
\hline $\begin{array}{l}\text { MF PF1 } \\
\text { P28 }\end{array}$ & $\begin{array}{c}47.26 \pm \\
0.530\end{array}$ & \multirow{2}{*}{$p=0.42$} & $\begin{array}{c}36.67 \pm \\
5.284 \\
(n=2 \\
\text { vesicles) }\end{array}$ & $0.019 \pm 0.013$ & \multirow{2}{*}{$* * p=0.003$} & & \\
\hline $\begin{array}{l}\text { MF PF2 } \\
\text { P28 }\end{array}$ & $\begin{array}{c}46.33 \pm \\
0.281\end{array}$ & & $\begin{array}{c}47.16 \pm \\
1.711 \\
(n=24 \\
\text { vesicles) }\end{array}$ & $0.137 \pm 0.036$ & & & \\
\hline \multicolumn{6}{|c|}{ Giant vesicles } & & \\
\hline $\begin{array}{l}\text { MF PF1 } \\
\text { P28 }\end{array}$ & $\begin{array}{c}88.30 \pm \\
6.890 \\
(n=11 \\
\text { vesicles) } \\
\end{array}$ & \multirow{2}{*}{$p=0.11$} & - & $\begin{array}{c}0 \pm 0(2 \\
\text { tomograms })\end{array}$ & & & \\
\hline $\begin{array}{l}\text { MF PF2 } \\
\text { P28 }\end{array}$ & $\begin{array}{c}76.17 \pm \\
3.658 \\
\text { ( } n=14 \\
\text { vesicles) }\end{array}$ & & $\begin{array}{c}62.78 \pm \\
2.022 \\
(n=3 \\
\text { vesicles) }\end{array}$ & $\begin{array}{c}16.67 \pm 9.796 \\
(11 \\
\text { tomograms })\end{array}$ & & & \\
\hline \multicolumn{6}{|c|}{ Dense-core vesicles } & & \\
\hline $\begin{array}{l}\text { MF PF1 } \\
\text { P28 }\end{array}$ & $\begin{array}{c}61.34 \pm \\
3.613 \\
\text { ( } n=14 \\
\text { vesicles) }\end{array}$ & \multirow{2}{*}{$p=0.28$} & - & & & & \\
\hline $\begin{array}{l}\text { MF PF2 } \\
\text { P28 }\end{array}$ & $\begin{array}{c}75.01 \pm \\
9.794 \\
(n=6 \\
\text { vesicles) }\end{array}$ & & $\begin{array}{c}74.70 \pm \\
5.170 \\
(\mathrm{n}=9 \\
\text { vesicles) }\end{array}$ & & & & \\
\hline \multicolumn{8}{|c|}{$\begin{array}{l}\text { Abbreviations: AZ, Active zone; } M F \text {, mossy fiber; PF1, perfusion fixation protocol } 1 \text { (ice-cold } 4 \% \text { PFA, } 2.5 \% \text { GA in } 0.1 \mathrm{M} \mathrm{PB} \text {, } \\
\text { pH 7.4) (Rollenhagen et al., 2007); PF2, perfusion fixation protocol } 2\left(37^{\circ} \mathrm{C} 2 \% \mathrm{PFA}, 2.5 \% \mathrm{GA}, 2 \mathrm{mM} \mathrm{CaCl} 2 \text { in } 0.1 \mathrm{M}\right. \\
\text { cacodylate buffer, } \mathrm{pH} \text { 7.4) (Chicurel and Harris, } 1992) \text {. P-values in green are from normally distributed data; } p \text {-values in } \\
\text { blue are from non-normally distributed data. } P 28 \text { wild-type mice were perfusion-fixed in this study. }\end{array}$} \\
\hline
\end{tabular}


Table 23. Statistical analysis of vesicle distributions in mossy fiber synapses from Munc13deficient and control slice cultures high-pressure frozen at DIV28.

\begin{tabular}{|c|c|c|c|c|c|c|c|c|}
\hline $\begin{array}{l}\text { Mossy } \\
\text { Fiber } \\
\text { Munc13- } \\
1 / 2\end{array}$ & \multicolumn{2}{|c|}{$\begin{array}{l}\text { Docked vesicles } \\
(0-2 \mathrm{~nm}) \text { per } 0.01 \mu \mathrm{m}^{2} \\
\text { AZ area }\end{array}$} & \multicolumn{2}{|c|}{$\begin{array}{l}\text { Vesicles }(0-20 \mathrm{~nm}) \text { per } \\
0.01 \mu^{2} \mathrm{AZ} \text { area }\end{array}$} & \multicolumn{2}{|c|}{$\begin{array}{l}\text { Vesicles }(0-40 \mathrm{~nm}) \text { per } \\
0.01 \mu \mathrm{m}^{2} \mathrm{AZ} \text { area }\end{array}$} & \multicolumn{2}{|c|}{$\begin{array}{l}\text { Vesicles }(0-100 \mathrm{~nm}) \text { per } \\
0.01 \mu \mathrm{m}^{2} \mathrm{AZ} \text { area }\end{array}$} \\
\hline \multicolumn{9}{|c|}{ Synaptic vesicles } \\
\hline $\begin{array}{l}\text { MF CTRL } \\
\text { DIV28 }\end{array}$ & $\begin{array}{c}0.810 \pm \\
0.149 \\
\end{array}$ & \multirow{2}{*}{$\begin{array}{c}* * * \\
\mathrm{p}<0.001\end{array}$} & $\begin{array}{c}1.285 \pm \\
0.217 \\
\end{array}$ & \multirow{2}{*}{$* p=0.04$} & $\begin{array}{c}1.704 \pm \\
0.271 \\
\end{array}$ & \multirow{2}{*}{$p=0.18$} & $\begin{array}{c}5.466 \pm \\
0.763 \\
\end{array}$ & \multirow{2}{*}{$p=0.43$} \\
\hline $\begin{array}{l}\text { MF DKO } \\
\text { DIV28 }\end{array}$ & $0 \pm 0$ & & $\begin{array}{c}0.795 \pm \\
0.074 \\
\end{array}$ & & $\begin{array}{c}2.134 \pm \\
0.155\end{array}$ & & $\begin{array}{c}6.184 \pm \\
0.487 \\
\end{array}$ & \\
\hline \multicolumn{9}{|c|}{ Synaptic vesicles and giant vesicles } \\
\hline $\begin{array}{l}\text { MF CTRL } \\
\text { DIV28 }\end{array}$ & $\begin{array}{c}0.861 \pm \\
0.159 \\
\end{array}$ & \multirow{2}{*}{$\begin{array}{c}* * * \\
\mathrm{p}<0.001\end{array}$} & $\begin{array}{c}1.374 \pm \\
0.231 \\
\end{array}$ & \multirow[b]{2}{*}{$* p=0.05$} & $\begin{array}{c}1.903 \pm \\
0.253 \\
\end{array}$ & \multirow[b]{2}{*}{$p=0.19$} & $\begin{array}{c}5.983 \pm \\
0.728 \\
\end{array}$ & \multirow[b]{2}{*}{$p=0.55$} \\
\hline $\begin{array}{l}\text { MF DKO } \\
\text { DIV28 }\end{array}$ & $0 \pm 0$ & & $\begin{array}{c}0.885 \pm \\
0.057\end{array}$ & & $\begin{array}{c}2.303 \pm \\
0.156\end{array}$ & & $\begin{array}{c}6.499 \pm \\
0.461\end{array}$ & \\
\hline \multicolumn{9}{|c|}{ Giant vesicles } \\
\hline $\begin{array}{l}\text { MF CTRL } \\
\text { DIV28 }\end{array}$ & $\begin{array}{c}0.051 \pm \\
0.024 \\
\end{array}$ & \multirow{2}{*}{$p=0.10$} & $\begin{array}{c}0.089 \pm \\
0.031 \\
\end{array}$ & \multirow{2}{*}{$p<0.99$} & $\begin{array}{c}0.152 \pm \\
0.039 \\
\end{array}$ & \multirow{2}{*}{$p=0.86$} & $\begin{array}{c}0.229 \pm \\
0.058 \\
\end{array}$ & \multirow{2}{*}{$p=0.39$} \\
\hline $\begin{array}{l}\text { MF DKO } \\
\text { DIV28 }\end{array}$ & $0 \pm 0$ & & $\begin{array}{c}0.090 \pm \\
0.032 \\
\end{array}$ & & $\begin{array}{c}0.169 \pm \\
0.055\end{array}$ & & $\begin{array}{c}0.315 \pm \\
0.080\end{array}$ & \\
\hline \multicolumn{9}{|c|}{ Dense-core vesicles } \\
\hline $\begin{array}{l}\text { MF CTRL } \\
\text { DIV28 }\end{array}$ & $\begin{array}{c}0.077 \pm \\
0.035\end{array}$ & \multirow{2}{*}{$p=0.10$} & $\begin{array}{c}0.174 \pm \\
0.056\end{array}$ & \multirow{2}{*}{$p=0.58$} & $\begin{array}{c}0.191 \pm \\
0.054\end{array}$ & \multirow{2}{*}{$p=0.15$} & $\begin{array}{c}0.252 \pm \\
0.061\end{array}$ & \multirow{2}{*}{$* * p=0.002$} \\
\hline $\begin{array}{l}\text { MF DKO } \\
\text { DIV28 }\end{array}$ & $0 \pm 0$ & & $\begin{array}{c}0.210 \pm \\
0.050\end{array}$ & & $\begin{array}{c}0.343 \pm \\
0.072\end{array}$ & & $\begin{array}{c}0.643 \pm \\
0.095\end{array}$ & \\
\hline
\end{tabular}


Table 24. Statistical analysis of vesicle diameters in mossy fiber synapses from Munc13deficient and control slice cultures high-pressure frozen at DIV28.

\begin{tabular}{|c|c|c|c|c|c|c|c|}
\hline & \multicolumn{2}{|c|}{$\begin{array}{l}\text { Non-docked vesicle } \\
\text { diameter }(\mathrm{nm})\end{array}$} & $\begin{array}{l}\text { Docked vesicle } \\
\text { diameter }(\mathrm{nm})\end{array}$ & \multicolumn{2}{|c|}{$\begin{array}{l}\text { Proportion of docked } \\
\text { vesicles (\%) }\end{array}$} & \multicolumn{2}{|c|}{ AZ area $\left(0.01 \mu \mathrm{m}^{2}\right)$} \\
\hline \multicolumn{8}{|c|}{ Synaptic vesicles } \\
\hline $\begin{array}{l}\text { MF CTRL } \\
\text { DIV28 }\end{array}$ & $\begin{array}{l}46.42 \pm \\
0.366\end{array}$ & \multirow[t]{2}{*}{$p=0.15$} & $\begin{array}{c}48.23 \pm \\
0.540 \\
(n=67 \\
\text { vesicles) }\end{array}$ & $\begin{array}{l}95.01 \pm \\
2.686\end{array}$ & & $\begin{array}{c}4.615 \pm \\
0.387\end{array}$ & \multirow[t]{2}{*}{$p=0.51$} \\
\hline $\begin{array}{l}\text { MF DKO } \\
\text { DIV28 }\end{array}$ & $\begin{array}{l}47.15 \pm \\
0.340\end{array}$ & & - & - & & $\begin{array}{c}4.976 \pm \\
0.379\end{array}$ & \\
\hline \multicolumn{4}{|c|}{ Synaptic vesicles and giant vesicles } & \multicolumn{2}{|c|}{$\begin{array}{l}\text { Vesicles } 0-2 \mathrm{~nm} \text { per } \\
\text { vesicles } 0-40 \mathrm{~nm}\end{array}$} & & \\
\hline $\begin{array}{l}\text { MF CTRL } \\
\text { DIV28 }\end{array}$ & $\begin{array}{l}48.26 \pm \\
0.808\end{array}$ & \multirow[t]{2}{*}{$p=0.46$} & $\begin{array}{c}50.9 \pm 1.27 \\
(n=72 \\
\text { vesicles })\end{array}$ & $\begin{array}{l}0.429 \pm \\
0.052\end{array}$ & \multirow{2}{*}{$\begin{array}{c}* * * \\
\mathrm{p}<0.001\end{array}$} & & \\
\hline $\begin{array}{l}\text { MF DKO } \\
\text { DIV28 }\end{array}$ & $\begin{array}{l}49.18 \pm \\
1.006 \\
\end{array}$ & & - & $0 \pm 0$ & & & \\
\hline \multicolumn{6}{|c|}{ Giant vesicles } & & \\
\hline $\begin{array}{l}\text { MF CTRL } \\
\text { DIV28 }\end{array}$ & $\begin{array}{c}83.17 \pm \\
2.564 \\
(n=13 \\
\text { vesicles) }\end{array}$ & \multirow{2}{*}{$* p=0.03$} & $\begin{array}{c}86.78 \pm \\
1.83 \\
(n=5 \\
\text { vesicles) }\end{array}$ & $\begin{array}{l}4.994 \pm \\
2.686\end{array}$ & & & \\
\hline $\begin{array}{l}\text { MF DKO } \\
\text { DIV28 }\end{array}$ & $\begin{array}{c}75.50 \pm \\
2.55 \\
(n=23 \\
\text { vesicles) }\end{array}$ & & - & - & & & \\
\hline \multicolumn{6}{|c|}{ Dense-core vesicles } & & \\
\hline $\begin{array}{l}\text { MF CTRL } \\
\text { DIV28 }\end{array}$ & $\begin{array}{c}71.22 \pm \\
3.091 \\
\text { ( } n=13 \\
\text { vesicles) }\end{array}$ & \multirow{2}{*}{$p=0.98$} & $\begin{array}{c}69.85 \pm \\
4.09 \\
(n=4 \\
\text { vesicles) }\end{array}$ & & & & \\
\hline $\begin{array}{l}\text { MF DKO } \\
\text { DIV28 }\end{array}$ & $\begin{array}{c}71.31 \pm \\
1.318 \\
(n=45 \\
\text { vesicles) }\end{array}$ & & 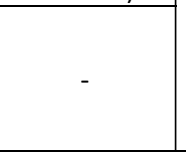 & & & & \\
\hline $\begin{array}{l}\text { Abbreviat } \\
\text { in green a }\end{array}$ & $\begin{array}{l}\text { AZ, Active } \\
\text { om norma }\end{array}$ & MF, $m$ & $\begin{array}{l}\text { per; CTRL, } M \\
\text { p-values in } k\end{array}$ & nc13-2 & Mur & $\mathrm{Mu}$ & lues \\
\hline
\end{tabular}


Table 25. Statistical analysis of vesicle distributions in mossy fiber-CA3 synapses after acute pharmacological silencing in slice cultures high-pressure frozen at DIV14.

\begin{tabular}{|c|c|c|c|c|c|c|c|c|}
\hline \multirow{2}{*}{\begin{tabular}{|l|l|l} 
& \\
Synaptic ves
\end{tabular}} & \multicolumn{2}{|c|}{$\begin{array}{l}\text { Docked vesicles }(0-2 \mathrm{~nm}) \\
\text { per } 0.01 \mu \mathrm{m}^{2} \mathrm{AZ} \text { area }\end{array}$} & \multicolumn{2}{|c|}{$\begin{array}{l}\text { Vesicles }(0-20 \mathrm{~nm}) \text { per } \\
0.01 \mu \mathrm{m}^{2} \mathrm{AZ} \text { area }\end{array}$} & \multicolumn{2}{|c|}{\begin{tabular}{|l|} 
Vesicles $(0-40 \mathrm{~nm})$ per \\
$0.01 \mu \mathrm{m}^{2} \mathrm{AZ}$ area \\
\end{tabular}} & \multicolumn{2}{|c|}{$\begin{array}{l}\text { Vesicles }(0-100 \mathrm{~nm}) \\
\text { per } 0.01 \mu \mathrm{m}^{2} \mathrm{AZ} \text { area }\end{array}$} \\
\hline & sicles & & & & & & & \\
\hline $\begin{array}{l}\text { MF VC } \\
\text { DIV14 }\end{array}$ & $\begin{array}{c}0.562 \pm \\
0.109\end{array}$ & \multirow{3}{*}{$p=0.69$} & $\begin{array}{l}1.508 \pm \\
0.206\end{array}$ & \multirow{3}{*}{$p=0.18$} & $\begin{array}{c}1.974 \pm \\
0.242 \\
\end{array}$ & \multirow{3}{*}{$p=0.54$} & $\begin{array}{c}6.061 \pm \\
0.599 \\
\end{array}$ & \multirow{3}{*}{$p=0.43$} \\
\hline $\begin{array}{l}\text { MF T/N/A } \\
\text { DIV14 }\end{array}$ & $\begin{array}{c}0.582 \pm \\
0.067\end{array}$ & & $\begin{array}{c}1.343 \pm \\
0.143\end{array}$ & & $\begin{array}{c}2.006 \pm \\
0.185\end{array}$ & & $\begin{array}{c}6.188 \pm \\
0.427\end{array}$ & \\
\hline $\begin{array}{l}\text { MF T/D } \\
\text { DIV14 }\end{array}$ & $\begin{array}{l}0.558 \pm \\
0.082\end{array}$ & & $\begin{array}{l}1.606 \pm \\
0.1239\end{array}$ & & $\begin{array}{l}2.084 \pm \\
0.1351\end{array}$ & & $\begin{array}{c}5.445 \pm \\
0.442\end{array}$ & \\
\hline \multicolumn{9}{|c|}{ Synaptic vesicles and giant vesicles } \\
\hline $\begin{array}{l}\text { MF VC } \\
\text { DIV14 }\end{array}$ & $\begin{array}{c}0.634 \pm \\
0.110\end{array}$ & \multirow{3}{*}{$\mathrm{p}=0.82$} & $\begin{array}{c}1.788 \pm \\
0.221\end{array}$ & \multirow{3}{*}{$p=0.05$} & $\begin{array}{c}2.318 \pm \\
0.261\end{array}$ & \multirow{3}{*}{$p=0.12$} & $\begin{array}{c}6.763 \pm \\
0.609\end{array}$ & \multirow{3}{*}{$p=0.62$} \\
\hline $\begin{array}{l}\text { MF T/N/A } \\
\text { DIV14 }\end{array}$ & $\begin{array}{l}0.605 \pm \\
0.066\end{array}$ & & $\begin{array}{c}1.626 \pm \\
0.149\end{array}$ & & $\begin{array}{r}2.389 \\
\pm 0.181 \\
\end{array}$ & & $\begin{array}{c}6.868 \pm \\
0.416 \\
\end{array}$ & \\
\hline $\begin{array}{l}\text { MF T/D } \\
\text { DIV14 }\end{array}$ & $\begin{array}{c}0.688 \pm \\
0.101\end{array}$ & & $\begin{array}{c}2.095 \pm \\
0.142\end{array}$ & & $\begin{array}{l}2.622 \pm \\
0.1421\end{array}$ & & $\begin{array}{c}6.390 \pm \\
0.419\end{array}$ & \\
\hline \multicolumn{9}{|c|}{ Giant vesicles } \\
\hline $\begin{array}{l}\text { MF VC } \\
\text { DIV14 }\end{array}$ & $\begin{array}{c}0.0411 \pm \\
0.028\end{array}$ & \multirow{3}{*}{$\begin{array}{l}* * \mathrm{p}=0.004 \\
* * \mathrm{~T} / \mathrm{N} / \mathrm{A} v \\
\quad \mathrm{~T} / \mathrm{D}\end{array}$} & $\begin{array}{c}0.280 \pm \\
0.054\end{array}$ & \multirow{3}{*}{$p=0.10$} & $\begin{array}{c}0.344 \pm \\
0.080\end{array}$ & \multirow{3}{*}{$p=0.17$} & $\begin{array}{c}0.702 \pm \\
0.132\end{array}$ & \multirow{3}{*}{$p=0.20$} \\
\hline $\begin{array}{l}\text { MF T/N/A } \\
\text { DIV14 }\end{array}$ & $\begin{array}{l}0.005 \pm \\
0.005\end{array}$ & & $\begin{array}{c}0.283 \pm \\
0.058\end{array}$ & & $\begin{array}{c}0.383 \pm \\
0.063\end{array}$ & & $\begin{array}{c}0.680 \pm \\
0.095\end{array}$ & \\
\hline $\begin{array}{l}\text { MF T/D } \\
\text { DIV14 }\end{array}$ & $\begin{array}{l}0.129 \pm \\
0.046\end{array}$ & & $\begin{array}{l}0.490 \pm \\
0.083\end{array}$ & & $\begin{array}{c}0.538 \pm \\
0.088\end{array}$ & & $\begin{array}{c}0.945 \pm \\
0.116\end{array}$ & \\
\hline \multicolumn{9}{|c|}{ Dense-core vesicles } \\
\hline $\begin{array}{l}\text { MF VC } \\
\text { DIV14 }\end{array}$ & $\begin{array}{c}0.014 \pm \\
0.010\end{array}$ & \multirow{3}{*}{$p=0.63$} & $\begin{array}{c}0.151 \pm \\
0.047\end{array}$ & \multirow{3}{*}{$\begin{array}{l}* \mathrm{p}=0.05 * \\
\mathrm{~T} / \mathrm{N} / \mathrm{A} v \\
\quad \mathrm{~T} / \mathrm{D}\end{array}$} & $\begin{array}{c}0.206 \pm \\
0.048\end{array}$ & \multirow{3}{*}{$\begin{array}{l}* p=0.05 \\
\text { all ns }\end{array}$} & $\begin{array}{c}0.342 \pm \\
0.060\end{array}$ & \multirow{3}{*}{$p=0.17$} \\
\hline $\begin{array}{l}\text { MF T/N/A } \\
\text { DIV14 }\end{array}$ & $\begin{array}{c}0.020 \pm \\
0.013\end{array}$ & & $\begin{array}{c}0.080 \pm \\
0.031\end{array}$ & & $\begin{array}{c}0.104 \pm \\
0.034\end{array}$ & & $\begin{array}{c}0.205 \pm \\
0.049\end{array}$ & \\
\hline $\begin{array}{l}\text { MF T/D } \\
\text { DIV14 }\end{array}$ & $\begin{array}{c}0.043 \pm \\
0.023\end{array}$ & & $\begin{array}{c}0.215 \pm \\
0.063\end{array}$ & & $\begin{array}{c}0.271 \pm \\
0.082\end{array}$ & & $\begin{array}{c}0.337 \pm \\
0.087\end{array}$ & \\
\hline \multicolumn{9}{|c|}{$\begin{array}{l}\text { Abbreviations: } A Z, \text { Active zone; } M F \text {, mossy fiber. Slices were treated for } 10 \text { minutes with either: VC (slice culture } \\
\text { medium), T/N/A (1 } \mu M T T X, 2 \mu M N B Q X \text {, and } 50 \mu M D-A P V) \text {, or T/D (1 } \mu M T T X \text { and } 2 \mu M D C G-I V) \text { prior to cryo-fixation. } \\
\text { P-values in green are from normally distributed data; } p \text {-values in blue are from non-normally distributed data. }\end{array}$} \\
\hline
\end{tabular}


Table 26. Statistical analysis of vesicle distributions in mossy fiber-CA3 synapses after acute pharmacological silencing in slice cultures high-pressure frozen at DIV14.

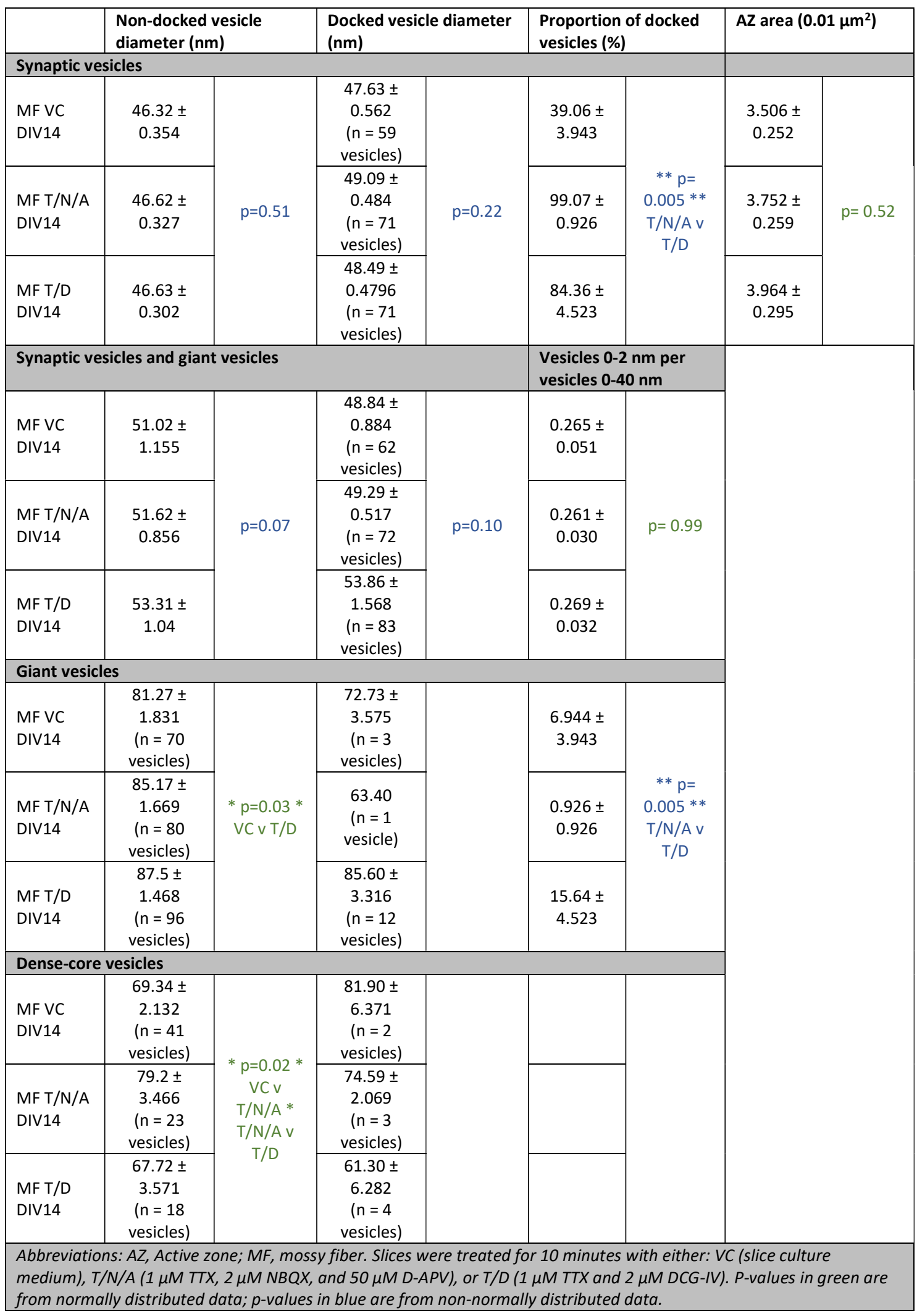


Table 27. Statistical analysis of vesicle distributions in mossy fiber synapses after pharmacological manipulation of presynaptic cAMP in slice cultures high-pressure frozen at DIV28.

\begin{tabular}{|c|c|c|c|c|c|c|c|c|}
\hline & \multicolumn{2}{|c|}{$\begin{array}{l}\text { Docked vesicles } \\
(0-2 \mathrm{~nm}) \text { per } 0.01 \mu \mathrm{m}^{2} \\
\text { AZ area }\end{array}$} & \multicolumn{2}{|c|}{$\begin{array}{l}\text { Vesicles }(0-20 \mathrm{~nm}) \text { per } \\
0.01 \mu \mathrm{m}^{2} \mathrm{AZ} \text { area }\end{array}$} & \multicolumn{2}{|c|}{$\begin{array}{l}\text { Vesicles }(0-40 \mathrm{~nm}) \text { per } \\
0.01 \mu \mathrm{m}^{2} \mathrm{AZ} \text { area }\end{array}$} & \multicolumn{2}{|c|}{$\begin{array}{l}\text { Vesicles }(0-100 \mathrm{~nm}) \text { per } \\
0.01 \mu \mathrm{m}^{2} \mathrm{AZ} \text { area }\end{array}$} \\
\hline \multicolumn{9}{|c|}{ Synaptic vesicles } \\
\hline MF VC & $1.350 \pm$ & \multirow{5}{*}{$p=0.56$} & $2.019 \pm$ & \multirow{5}{*}{$p=0.73$} & $2.583 \pm$ & \multirow{5}{*}{$p=0.65$} & $6.486 \pm$ & \multirow{5}{*}{$p=0.05$} \\
\hline DIV28 & 0.185 & & 0.232 & & 0.226 & & 0.459 & \\
\hline $\begin{array}{l}\text { MF T/F } \\
\text { DIV28 }\end{array}$ & $\begin{array}{l}1.492 \pm \\
0.123\end{array}$ & & $\begin{array}{c}2.171 \pm \\
0.172\end{array}$ & & $\begin{array}{c}2.560 \pm \\
0.164\end{array}$ & & $\begin{array}{c}5.579 \pm \\
0.314\end{array}$ & \\
\hline $\mathrm{MF} T / \mathrm{D}$ & $1.349 \pm$ & & $1.978 \pm$ & & $2.388 \pm$ & & $5.353 \pm$ & \\
\hline DIV28 & 0.128 & & 0.156 & & 0.206 & & 0.475 & \\
\hline \multicolumn{9}{|c|}{ Synaptic vesicles and giant vesicles } \\
\hline MF VC & $1.422 \pm$ & \multirow{6}{*}{$p=0.40$} & $2.285 \pm$ & \multirow{6}{*}{$p=0.90$} & $2.871 \pm$ & \multirow{6}{*}{$p=0.85$} & $7.047 \pm$ & \multirow{6}{*}{$\begin{array}{l}* \mathrm{p}=0.03 * \\
\mathrm{VC} \vee \mathrm{T} / \mathrm{D}\end{array}$} \\
\hline DIV28 & 0.183 & & 0.235 & & 0.233 & & 0.473 & \\
\hline $\mathrm{MF} \mathrm{T} / \mathrm{F}$ & $1.601 \pm$ & & $2.366 \pm$ & & $2.775 \pm$ & & $7.047 \pm$ & \\
\hline DIV28 & 0.118 & & 0.162 & & 0.151 & & 0.473 & \\
\hline $\mathrm{MF} T / \mathrm{D}$ & $1.399 \pm$ & & $2.256 \pm$ & & $2.713 \pm$ & & $5.912 \pm$ & \\
\hline DIV28 & 0.129 & & 0.145 & & 0.178 & & 0.299 & \\
\hline \multicolumn{9}{|c|}{ Giant vesicles } \\
\hline MF VC & $0.072 \pm$ & \multirow{6}{*}{$p=0.16$} & $0.267 \pm$ & \multirow{6}{*}{$p=0.68$} & $0.288 \pm$ & \multirow{6}{*}{$p=0.62$} & $0.561 \pm$ & \multirow{6}{*}{$p=0.093$} \\
\hline DIV28 & 0.026 & & 0.052 & & 0.055 & & 0.098 & \\
\hline $\mathrm{MF} \mathrm{T} / \mathrm{F}$ & $0.109 \pm$ & & $0.195 \pm$ & & $0.215 \pm$ & & $0.333 \pm$ & \\
\hline DIV28 & 0.024 & & 0.032 & & 0.030 & & 0.044 & \\
\hline $\mathrm{MF} \mathrm{T} / \mathrm{D}$ & $0.049 \pm$ & & $0.278 \pm$ & & $0.325 \pm$ & & $0.476 \pm$ & \\
\hline DIV28 & 0.018 & & 0.067 & & 0.075 & & 0.093 & \\
\hline \multicolumn{9}{|c|}{ Dense-core vesicles } \\
\hline MF VC & $0.045 \pm$ & \multirow{6}{*}{$\begin{array}{l}* \mathrm{p}=0.014 * \\
\mathrm{~T} / \mathrm{F} \vee \mathrm{T} / \mathrm{D}\end{array}$} & $0.175 \pm$ & \multirow{6}{*}{$p=0.73$} & $0.192 \pm$ & \multirow{6}{*}{$p=0.61$} & $0.328 \pm$ & \multirow{6}{*}{$p=0.19$} \\
\hline DIV28 & 0.019 & & 0.044 & & 0.045 & & 0.064 & \\
\hline $\mathrm{MF} \mathrm{T} / \mathrm{F}$ & $0.092 \pm$ & & $0.126 \pm$ & & $0.133 \pm$ & & $0.225 \pm$ & \\
\hline DIV28 & 0.022 & & 0.029 & & 0.028 & & 0.036 & \\
\hline $\mathrm{MF} T / \mathrm{D}$ & $0.014 \pm$ & & $0.133 \pm$ & & $0.141 \pm$ & & $0.170 \pm$ & \\
\hline DIV28 & 0.009 & & 0.041 & & 0.043 & & 0.044 & \\
\hline \multicolumn{9}{|c|}{$\begin{array}{l}\text { Abbreviations: AZ, Active zone; MF, mossy fiber. Slices were treated for } 15 \text { minutes with either: VC (1 } \mu M T T X), T / F(1 \mu M \\
T T X \text { and } 25 \mu M \text { forskolin), or T/D (1 } 1 M \text { MTX and } 2 \mu M \text { DCG-IV). P-values in green are from normally distributed data; } p \text { - } \\
\text { values in blue are from non-normally distributed data. }\end{array}$} \\
\hline
\end{tabular}


Table 28. Statistical analysis of vesicle diameters in mossy fiber synapses after pharmacological manipulation of presynaptic CAMP in slice cultures high-pressure frozen at DIV28.

\begin{tabular}{|c|c|c|c|c|c|c|c|c|}
\hline & \multicolumn{2}{|c|}{$\begin{array}{l}\text { Non-docked vesicle } \\
\text { diameter }(\mathrm{nm})\end{array}$} & \multicolumn{2}{|c|}{$\begin{array}{l}\text { Docked vesicle diameter } \\
(\mathrm{nm})\end{array}$} & \multicolumn{2}{|c|}{$\begin{array}{l}\text { Proportion of docked } \\
\text { vesicles (\%) }\end{array}$} & \multicolumn{2}{|c|}{$A Z$ area $\left(0.01 \mu m^{2}\right)$} \\
\hline \multicolumn{9}{|c|}{ Synaptic vesicles } \\
\hline $\begin{array}{l}\text { MF VC } \\
\text { DIV28 }\end{array}$ & $\begin{array}{l}46.72 \pm \\
0.306\end{array}$ & \multirow{3}{*}{$p=0.23$} & $\begin{array}{c}47.8 \pm \\
0.330 \\
(n=158 \\
\text { vesicles) }\end{array}$ & \multirow{3}{*}{$p=0.95$} & $\begin{array}{c}91.76 \pm \\
4.179\end{array}$ & \multirow{3}{*}{$p=0.09$} & $\begin{array}{l}4.689 \pm \\
0.292\end{array}$ & \multirow{3}{*}{$\begin{array}{c}* * * \\
\mathrm{p}<0.001 \\
* * * \vee C \vee \\
\mathrm{T} / \mathrm{F} \\
* \mathrm{~T} / \mathrm{F} \vee \mathrm{T} / \mathrm{D}\end{array}$} \\
\hline $\begin{array}{l}\mathrm{MFT} / \mathrm{F} \\
\mathrm{DIV} 28\end{array}$ & $\begin{array}{c}46.59 \pm \\
0.224\end{array}$ & & $\begin{array}{c}47.69 \pm \\
0.196 \\
(n=331 \\
\text { vesicles) }\end{array}$ & & $\begin{array}{c}91.99 \pm \\
1.677\end{array}$ & & $\begin{array}{c}6.789 \pm \\
0.402\end{array}$ & \\
\hline $\begin{array}{l}\text { MF T/D } \\
\text { DIV28 }\end{array}$ & $\begin{array}{l}47.2 \pm \\
0.250\end{array}$ & & $\begin{array}{c}47.98 \pm \\
0.277 \\
\text { ( } n=170 \\
\text { vesicles) }\end{array}$ & & $\begin{array}{c}96.50 \pm \\
1.420\end{array}$ & & $\begin{array}{c}5.461 \pm \\
0.346\end{array}$ & \\
\hline \multicolumn{5}{|c|}{ Synaptic vesicles and giant vesicles } & \multicolumn{2}{|c|}{$\begin{array}{l}\text { Vesicles } 0-2 \mathrm{~nm} \text { per } \\
\text { vesicles } 0-40 \mathrm{~nm}\end{array}$} & & \\
\hline $\begin{array}{l}\text { MF VC } \\
\text { DIV28 }\end{array}$ & $\begin{array}{c}50.25 \pm \\
0.767\end{array}$ & \multirow{3}{*}{$p=0.16$} & $\begin{array}{c}49.26 \pm \\
0.631 \\
(n=166 \\
\text { vesicles) }\end{array}$ & \multirow{3}{*}{$p=0.94$} & $\begin{array}{l}0.4822 \pm \\
0.044\end{array}$ & \multirow{3}{*}{$p=0.08$} & & \\
\hline $\begin{array}{l}\text { MF T/F } \\
\text { DIV28 }\end{array}$ & $\begin{array}{c}49.07 \pm \\
0.458\end{array}$ & & $\begin{array}{c}50.69 \pm \\
0.662 \\
(n=355 \\
\text { vesicles) }\end{array}$ & & $\begin{array}{c}0.589 \pm \\
0.031\end{array}$ & & & \\
\hline $\begin{array}{l}\text { MF T/D } \\
\text { DIV28 }\end{array}$ & $\begin{array}{l}51.92 \pm \\
1.083\end{array}$ & & $\begin{array}{c}49.68 \pm \\
0.754 \\
(n=176 \\
\text { vesicles) }\end{array}$ & & $\begin{array}{l}0.517 \pm \\
0.033\end{array}$ & & & \\
\hline \multicolumn{7}{|c|}{ Giant vesicles } & & \\
\hline $\begin{array}{l}\text { MF VC } \\
\text { DIV28 }\end{array}$ & $\begin{array}{c}85.77 \pm \\
2.248 \\
(n=55 \\
\text { vesicles) }\end{array}$ & \multirow{3}{*}{$p=0.57$} & $\begin{array}{c}78.05 \pm \\
4.729 \\
(n=8 \\
\text { vesicles) }\end{array}$ & & $\begin{array}{c}8.241 \pm \\
4.179\end{array}$ & \multirow{3}{*}{$p=0.09$} & & \\
\hline $\begin{array}{l}\mathrm{MFT} / \mathrm{F} \\
\mathrm{DIV} 28\end{array}$ & $\begin{array}{c}84.13 \pm \\
2.23 \\
(n=50 \\
\text { vesicles) }\end{array}$ & & $\begin{array}{c}91.98 \pm \\
3.557 \\
(n=24 \\
\text { vesicles) }\end{array}$ & & $\begin{array}{c}8.012 \pm \\
1.677\end{array}$ & & & \\
\hline $\begin{array}{l}\text { MF T/D } \\
\text { DIV28 }\end{array}$ & $\begin{array}{c}88.14 \pm \\
2.192 \\
(n=52 \\
\text { vesicles) }\end{array}$ & & $\begin{array}{c}97.75 \pm \\
5.611 \\
(\mathrm{n}=6 \\
\text { vesicles) }\end{array}$ & & $\begin{array}{c}3.504 \pm \\
1.420\end{array}$ & & & \\
\hline \multicolumn{7}{|c|}{ Dense-core vesicles } & & \\
\hline $\begin{array}{l}\text { MF VC } \\
\text { DIV28 }\end{array}$ & $\begin{array}{c}72.25 \pm \\
2.808 \\
(n=33 \\
\text { vesicles) }\end{array}$ & \multirow{3}{*}{$p=0.98$} & $\begin{array}{c}63.47 \pm \\
5.316 \\
(n=5 \\
\text { vesicles) }\end{array}$ & & & & & \\
\hline $\begin{array}{l}\mathrm{MFT} / \mathrm{F} \\
\mathrm{DIV} 28\end{array}$ & $\begin{array}{c}72.84 \pm \\
2.771 \\
(n=31 \\
\text { vesicles) }\end{array}$ & & $\begin{array}{c}72.36 \pm \\
4.187 \\
\text { ( } n=21 \\
\text { vesicles) }\end{array}$ & & & & & \\
\hline $\begin{array}{l}\text { MF T/D } \\
\text { DIV28 }\end{array}$ & $\begin{array}{c}2.21 \pm \\
1.831 \\
(n=21 \\
\text { vesicles) }\end{array}$ & & $\begin{array}{c}55.17 \pm \\
0.155 \\
(\mathrm{n}=2 \\
\text { vesicles) }\end{array}$ & & & & & \\
\hline \multicolumn{9}{|c|}{$\begin{array}{l}\text { Abbreviations: MF, mossy fiber. Slices were treated for } 15 \text { minutes with either: VC (1 } \mu M T T X), T / F(1 \mu M T T X \text { and } 25 \mu M \\
\text { forskolin), or T/D ( } 1 \mu M \text { TTX and } 2 \mu M D C G-I V) \text {. P-values in green are from normally distributed data; } p \text {-values in blue are } \\
\text { from non-normally distributed data. }\end{array}$} \\
\hline
\end{tabular}


Table 29. Statistical analysis of mEPSCs in CA3 pyramidal neurons recorded in slice culture at DIV14.

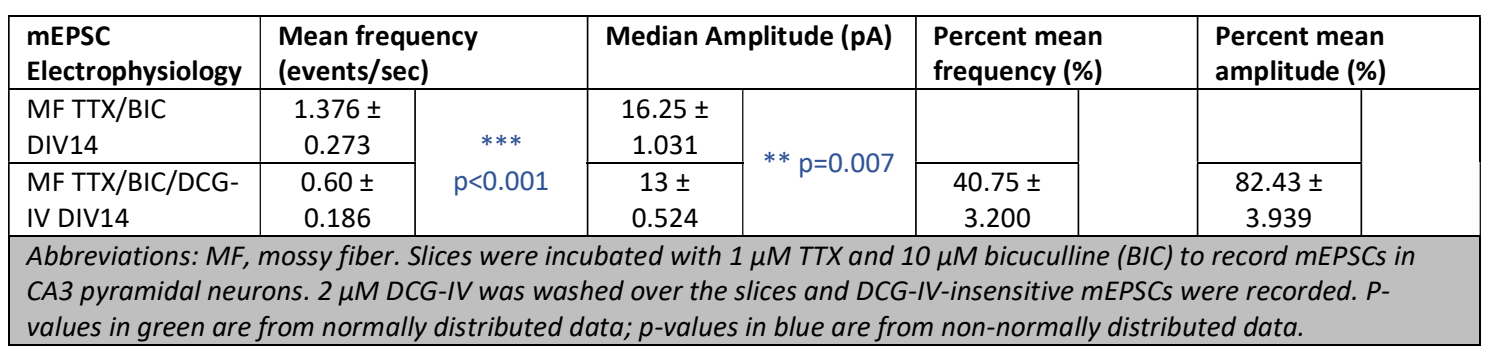


Table 30. Statistical analysis of docked vesicle distributions and diameters in mossy fiber synapses 5-11 $\mu \mathrm{m}$ from the tissue surface in slice cultures immersion-fixed at DIV28.

\begin{tabular}{|c|c|c|c|c|c|c|}
\hline Superficial & \multicolumn{2}{|c|}{$\begin{array}{l}\text { Docked vesicles }(0-2 \mathrm{~nm}) \text { per } \\
0.01 \mu \mathrm{m}^{2} \mathrm{AZ} \text { area }\end{array}$} & \multicolumn{2}{|c|}{ Docked Vesicle Diameter (nm) } & \multicolumn{2}{|c|}{$\begin{array}{l}\text { Proportion of docked vesicles } \\
\text { (\%) }\end{array}$} \\
\hline \multicolumn{7}{|c|}{ Synaptic vesicles } \\
\hline MF HPF DIV29 & $1.048 \pm 0.115$ & \multirow{3}{*}{$\begin{array}{c}p=0.53 \subset \vee \text { IF1 } \\
p=0.18 C \vee \text { IF2 } \\
p=0.07 \text { IF1 v } \\
\text { IF2 }\end{array}$} & $\begin{array}{l}49.65 \pm 0.304 \\
\text { (154 vesicles) }\end{array}$ & \multirow{3}{*}{$\begin{array}{c}* * * p<0.001 \mathrm{C} \\
v \mathrm{IF} 1 \mathrm{p}=0.76 \mathrm{C} \\
\text { v IF2 } * * \\
\mathrm{p}=0.004 \text { IF1 v } \\
\text { IF2 }\end{array}$} & $89.29 \pm 3.996$ & \multirow{3}{*}{$\begin{array}{c}p=0.79 \subset \vee \text { IF } 1 \\
p=0.58 \subset \vee \text { IF2 } \\
p=0.46 \text { IF1 v } \\
\text { IF2 }\end{array}$} \\
\hline MF IF1 DIV28 & $\begin{array}{l}0.9437 \pm \\
0.118 \\
\end{array}$ & & $\begin{array}{l}47.69 \pm 0.371 \\
\text { (112 vesicles) }\end{array}$ & & $89.54 \pm 3.505$ & \\
\hline MF IF2 DIV28 & $1.304 \pm 0.159$ & & $\begin{array}{c}49.31 \pm 0.414 \\
(118 \text { vesicle) }\end{array}$ & & $93.19 \pm 3.696$ & \\
\hline \multicolumn{5}{|c|}{ Synaptic vesicles and giant vesicles } & \multicolumn{2}{|c|}{ AZ area $\left(0.01 \mu \mathrm{m}^{2}\right)$} \\
\hline MF HPF DIV29 & $1.135 \pm 0.111$ & \multirow{3}{*}{$\begin{array}{c}\mathrm{p}=0.68 \subset \vee \text { IF1 } \\
\mathrm{p}=0.20 \mathrm{C} \vee \text { IF2 } \\
\mathrm{p}=0.12 \text { IF1 v } \\
\text { IF2 }\end{array}$} & $\begin{array}{l}52.11 \pm 0.805 \\
(165 \text { vesicles) }\end{array}$ & \multirow{3}{*}{$\begin{array}{c}* * p=0.006 \mathrm{CV} \\
\text { IF1 } \\
p=0.57 \mathrm{C} v \text { IF2 } \\
p=0.06 \text { IF1 v } \\
\text { IF2 }\end{array}$} & $4.544 \pm 0.219$ & \multirow{3}{*}{$\begin{array}{c}p=0.87 \subset \vee \text { IF1 } \\
p=0.09 C \vee \text { IF2 } \\
p=0.15 \text { IF1 } v \\
\text { IF2 }\end{array}$} \\
\hline MF IF1 DIV28 & $1.065 \pm 0.121$ & & $\begin{array}{l}51.34 \pm 1.085 \\
\text { (125 vesicles) }\end{array}$ & & $4.489 \pm 0.261$ & \\
\hline MF IF2 DIV28 & $1.373 \pm 0.152$ & & $\begin{array}{l}50.60 \pm 0.689 \\
\text { (124 vesicles) }\end{array}$ & & $3.935 \pm 0.279$ & \\
\hline \multicolumn{7}{|l|}{ Giant vesicles } \\
\hline MF HPF DIV29 & $\begin{array}{c}0.0864 \pm \\
0.0340 \\
\end{array}$ & \multirow{3}{*}{$\begin{array}{c}p=0.65 C \vee \text { IF1 } \\
p=0.65 C \vee \text { IF2 } \\
p=0.43 \text { IF1 v } \\
\text { IF2 }\end{array}$} & $\begin{array}{c}86.63 \pm 3.479 \\
(11 \text { vesicles) } \\
\end{array}$ & \multirow{3}{*}{$\begin{array}{c}p=0.64 \text { C v IF1 } \\
* p=0.04 \text { C v } \\
\text { IF2 } p=0.31 \text { IF1 } \\
\text { v IF2 }\end{array}$} & $10.71 \pm 3.996$ & \multirow{3}{*}{$\begin{array}{c}p=0.79 \subset \vee \text { IF } 1 \\
p=0.58 \subset \vee \text { IF2 } \\
p=0.46 \text { IF } 1 \vee \\
\text { IF2 }\end{array}$} \\
\hline MF IF1 DIV28 & $\begin{array}{l}0.122 \pm \\
0.0429 \\
\end{array}$ & & $\begin{array}{c}82.77 \pm 3.747 \\
(13 \text { vesicles }) \\
\end{array}$ & & $10.46 \pm 3.505$ & \\
\hline MF IF2 DIV28 & $\begin{array}{l}0.0684 \pm \\
0.0349 \\
\end{array}$ & & $\begin{array}{c}75.89 \pm 5.336 \\
\text { (6 vesicles) }\end{array}$ & & $6.807 \pm 3.696$ & \\
\hline \multicolumn{7}{|l|}{ DCVs } \\
\hline MF HPF DIV29 & $0.067 \pm 0.020$ & \multirow{3}{*}{$p=0.95 C \vee$ IF2 } & $\begin{array}{c}81.96 \pm 3.751 \\
(10 \text { vesicles }) \\
\end{array}$ & \multirow{3}{*}{$p=0.91 C \vee$ IF2 } & & \\
\hline MF IF1 DIV28 & $0.007 \pm 0.007$ & & $\begin{array}{l}76.77 \pm 0(1 \\
\text { vesicle) }\end{array}$ & & & \\
\hline MF IF2 DIV28 & $0.069 \pm 0.033$ & & $\begin{array}{c}81.27 \pm 4.916 \\
\text { (6 vesicles) }\end{array}$ & & & \\
\hline \multicolumn{7}{|c|}{$\begin{array}{l}\text { Abbreviations: AZ, Active zone; MF, mossy fiber; IF1, immersion fixation protocol } 1 \text { (ice-cold } 4 \% \text { PFA, } 2.5 \% \text { GA in } 0.1 \mathrm{M} \\
P B, p H \text { 7.4) (Rollenhagen et al., 2007); IF2, immersion fixation protocol } 2\left(37^{\circ} \mathrm{C} 2 \% \text { PFA, } 2.5 \% \mathrm{GA}, 2 \mathrm{mM} \mathrm{CaCl},{ }_{2} \text { in } 0.1 \mathrm{M}\right. \\
\text { cacodylate buffer, } \mathrm{pH} \text { 7.4) (Chicurel and Harris, 1992). P-values in green are from normally distributed data; } \text {-values in } \\
\text { blue are from non-normally distributed data. }\end{array}$} \\
\hline
\end{tabular}


Table 31. Statistical analysis of docked vesicle distributions and diameters in mossy fiber synapses 20-22 $\mu \mathrm{m}$ from the tissue surface in slice cultures immersion-fixed at DIV28.

\begin{tabular}{|c|c|c|c|c|c|c|}
\hline Deep & \multicolumn{2}{|c|}{$\begin{array}{l}\text { Docked vesicles }(0-2 \mathrm{~nm}) \text { per } \\
0.01 \mu \mathrm{m}^{2} \mathrm{AZ} \text { area }\end{array}$} & \multicolumn{2}{|c|}{ Docked Vesicle Diameter (nm) } & \multicolumn{2}{|c|}{$\begin{array}{l}\text { Proportion of docked vesicles } \\
\text { (\%) }\end{array}$} \\
\hline \multicolumn{7}{|c|}{ Synaptic vesicles } \\
\hline MF HPF DIV29 & $1.130 \pm 0.200$ & \multirow{3}{*}{$\begin{array}{c}p=0.34 \mathrm{C} \vee \text { IF1 } \\
* * p=0.006 \mathrm{C} v \\
\text { IF2 } * p=0.05 \\
\text { IF1 } v \text { IF2 }\end{array}$} & $\begin{array}{l}49.81 \pm 0.413 \\
\text { (88 vesicles) }\end{array}$ & \multirow{3}{*}{$\begin{array}{c}* * * p<0.001 \mathrm{C} \\
v \mathrm{IF} 1 * * \\
\mathrm{p}=0.002 \mathrm{C} v \\
\text { IF2 } \mathrm{p}=0.64 \text { IF1 } \\
\text { v IF2 }\end{array}$} & $65.70 \pm 8.203$ & \multirow{3}{*}{$\begin{array}{c}* p=0.02 \mathrm{Cv} \\
\text { IF1 } p=0.93 \mathrm{Cv} \\
\text { IF2 } p=0.08 \mathrm{~F} 1 \\
\text { v IF2 }\end{array}$} \\
\hline MF IF1 DIV28 & $0.864 \pm 0.174$ & & $\begin{array}{l}46.67 \pm 0.468 \\
\text { (63 vesicles) } \\
\end{array}$ & & $89.71 \pm 3.506$ & \\
\hline MF IF2 DIV28 & $\begin{array}{l}0.475 \pm \\
0.0882\end{array}$ & & $\begin{array}{l}7.10 \pm 0.911 \\
\text { (34 vesicles) }\end{array}$ & & $65.78 \pm 9.076$ & \\
\hline \multicolumn{5}{|c|}{ Synaptic vesicles and giant vesicles } & \multicolumn{2}{|c|}{$A Z$ area $\left(0.01 \mu \mathrm{m}^{2}\right)$} \\
\hline MF HPF DIV29 & $1.503 \pm 0.209$ & \multirow{3}{*}{$\begin{array}{c}p=0.09 \text { C v IF1 } \\
* * p=0.004 \text { C v } \\
\text { IF2 } p=0.35 \text { IF1 } \\
\text { v IF2 }\end{array}$} & $\begin{array}{c}59.66 \pm 1.63 \\
(119 \text { vesicles) }\end{array}$ & \multirow{3}{*}{$\begin{array}{c}* * * p<0.001 C \\
\text { v IF1 } p=0.37 C \\
\text { v IF2 } * p=0.01 \\
\text { IF1 v IF2 }\end{array}$} & $4.567 \pm 0.304$ & \multirow{3}{*}{$\begin{array}{c}p=0.59 C \vee \text { IF } 1 \\
p=0.06 C \vee \text { IF2 } \\
p=0.13 \text { IF } 1 \vee \\
\text { IF } 2\end{array}$} \\
\hline MF IF1 DIV28 & $0.981 \pm 0.201$ & & $\begin{array}{l}51.33 \pm 1.74 \\
\text { (71 vesicles) } \\
\end{array}$ & & $3.896 \pm 0.307$ & \\
\hline MF IF2 DIV28 & $0.777 \pm 0.097$ & & $\begin{array}{l}62.02 \pm 3.38 \\
\text { (50 vesicles) }\end{array}$ & & $4.839 \pm 0.374$ & \\
\hline \multicolumn{7}{|l|}{ Giant vesicles } \\
\hline MF HPF DIV29 & $0.373 \pm 0.063$ & \multirow{3}{*}{$\begin{array}{c}* * p=0.003 C v \\
\text { IF1 } p=0.21 C v \\
\text { IF2 } p=0.18 \text { IF1 } \\
v \text { IF2 }\end{array}$} & $\begin{array}{c}87.61 \pm 1.875 \\
\text { (31 vesicles) }\end{array}$ & \multirow{3}{*}{$\begin{array}{c}\mathrm{p}=0.94 \text { C } \vee \text { IF1 } \\
\mathrm{p}=0.19 \text { C } \vee \text { IF2 } \\
\mathrm{p}=0.31 \mathrm{IF} 1 \mathrm{v} \\
\text { IF2 }\end{array}$} & $34.30 \pm 8.203$ & \multirow{3}{*}{$\begin{array}{c}* p=0.02 C v \\
\text { IF1 } p=0.93 C v \\
\text { IF2 } p=0.08 \text { IF1 } \\
\text { v IF2 }\end{array}$} \\
\hline MF IF1 DIV28 & $0.116 \pm 0.041$ & & $\begin{array}{c}87.96 \pm 6.112 \\
\text { (8 vesicles) }\end{array}$ & & $10.29 \pm 3.506$ & \\
\hline MF IF2 DIV28 & $0.302 \pm 0.106$ & & $\begin{array}{c}93.71 \pm 3.743 \\
\text { (16 vesicles) } \\
\end{array}$ & & $34.22 \pm 9.076$ & \\
\hline \multicolumn{7}{|c|}{ Dense-core vesicles } \\
\hline MF HPF DIV29 & $\begin{array}{l}0.210 \pm \\
0.0620\end{array}$ & \multirow{3}{*}{$\begin{array}{c}* p=0.02 C v \\
\text { IF1 } p=0.42 C v \\
\text { IF2 } p=0.14 \text { IF1 } \\
v \text { IF2 }\end{array}$} & $\begin{array}{c}79.49 \pm 1.837 \\
\text { (16 vesicles) }\end{array}$ & \multirow{3}{*}{$p=0.84 C \vee$ IF2 } & & \\
\hline MF IF1 DIV28 & $0.049 \pm 0.035$ & & $\begin{array}{c}90.03 \pm 5.944 \\
\text { (3 vesicles) }\end{array}$ & & & \\
\hline MF IF2 DIV28 & $0.113 \pm 0.035$ & & $\begin{array}{c}80.28 \pm 4.269 \\
\text { (7 vesicles) }\end{array}$ & & & \\
\hline \multicolumn{7}{|c|}{$\begin{array}{l}\text { Abbreviations: AZ, Active zone; MF, mossy fiber; IF1, immersion fixation protocol } 1 \text { (ice-cold } 4 \% \text { PFA, } 2.5 \% \text { GA in } 0.1 \mathrm{M} \\
P B, p H \text { 7.4) (Rollenhagen et al., 2007); IF2, immersion fixation protocol } 2\left(37^{\circ} \mathrm{C} 2 \% \text { PFA, } 2.5 \% \mathrm{GA}, 2 \mathrm{mM} \mathrm{CaCl},{ }_{2} \text { in } 0.1 \mathrm{M}\right. \\
\text { cacodylate buffer, } \mathrm{pH} \text { 7.4) (Chicurel and Harris, 1992). P-values in green are from normally distributed data; } \text {-values in } \\
\text { blue are from non-normally distributed data. }\end{array}$} \\
\hline
\end{tabular}


Table 32. Statistical analysis of mossy fiber boutons imaged with STED microscopy in slice cultures after acute forskolin or control treatments at DIV28.

\begin{tabular}{|c|c|c|c|c|c|c|}
\hline \multirow{2}{*}{$\begin{array}{l}\text { STED } \\
\text { microscopy } \\
\text { MF VC DIV28 }\end{array}$} & \multicolumn{2}{|c|}{$\begin{array}{l}\text { Synaptoporin occupancy (\% of } \\
\text { vehicle control) }\end{array}$} & \multicolumn{2}{|c|}{$\begin{array}{l}\text { Bassoon area/synaptoporin } \\
\text { area (\% of vehicle control) }\end{array}$} & \multicolumn{2}{|c|}{$\begin{array}{l}\text { Bassoon area/synaptoporin } \\
\text { area }\end{array}$} \\
\hline & $100 \pm 3.612$ & \multirow{2}{*}{$* p=0.03$} & $100 \pm 7.423$ & \multirow{2}{*}{$p=0.08$} & $0.9276 \pm 0.069$ & \multirow{2}{*}{$p=0.08$} \\
\hline MF T/D DIV28 & $84.99 \pm 5.192$ & & $126.4 \pm 11.88$ & & $1.173 \pm 0.110$ & \\
\hline \multicolumn{7}{|c|}{$\begin{array}{l}\text { Abbreviations: MF, mossy fiber. Slices were treated for } 15 \text { minutes with either: VC ( } 1 \mu M \text { TTX) or T/F (1 } \mu M \text { TTX and } 25 \\
\mu M \text { forskolin). P-values in green are from normally distributed data; } p \text {-values in blue are from non-normally distributed } \\
\text { data. }\end{array}$} \\
\hline
\end{tabular}


Table 34. Statistical analysis of 2D presynaptic ultrastructural features of Schaffer collateral and mossy fiber synapses in slice cultures high-pressure frozen at DIV28

\begin{tabular}{|c|c|c|c|c|}
\hline 2D WT & \multicolumn{2}{|l|}{ Bouton } & \multicolumn{2}{|l|}{ Mitochondria } \\
\hline \multicolumn{3}{|l|}{ Perimeter } & \multicolumn{2}{|c|}{ Occupancy of bouton area (\%) } \\
\hline SC Unt DIV28 & $2.117 \pm 0.039$ & \multirow{2}{*}{$* * * p<0.001$} & $\begin{array}{l}15.29 \pm 1.071 \\
\text { (52 boutons) }\end{array}$ & \multirow{2}{*}{$* * * p<0.001$} \\
\hline MF Unt DIV28 & $11.37 \pm 0.449$ & & $\begin{array}{l}8.024 \pm 0.341 \\
\text { (130 boutons) }\end{array}$ & \\
\hline \multicolumn{5}{|l|}{ Area $\left(\mu \mathrm{m}^{2}\right)$} \\
\hline SC Unt DIV28 & $0.326 \pm 0.010$ & \multirow{2}{*}{$* * * p<0.001$} & $\begin{array}{l}0.057 \pm 0.004 \\
\text { (52 boutons) }\end{array}$ & \multirow{2}{*}{$* * * p<0.001$} \\
\hline MF Unt DIV28 & $4.446 \pm 0.245$ & & $\begin{array}{l}0.376 \pm 0.028 \\
\text { (130 boutons) }\end{array}$ & \\
\hline \multicolumn{5}{|c|}{ Perimeter/area $\left(\mu \mathrm{m}^{-1}\right)$} \\
\hline SC Unt DIV28 & $6.939 \pm 0.0114$ & \multirow{2}{*}{$* * * p<0.001$} & $\begin{array}{l}18.82 \pm 0.665 \\
\text { ( } 52 \text { boutons) }\end{array}$ & \multirow{2}{*}{$p=0.07$} \\
\hline MF Unt DIV28 & $2.961 \pm 0.085$ & & $\begin{array}{l}17.51 \pm 0.244 \\
\text { (130 boutons) }\end{array}$ & \\
\hline \multicolumn{5}{|c|}{ Proportion of boutons with mitochondria } \\
\hline SC Unt DIV28 & & & $\begin{array}{l}0.377 \pm 0.041 \\
\text { (52 boutons) }\end{array}$ & \multirow{2}{*}{$* * * p<0.001$} \\
\hline MF Unt DIV28 & & & $\begin{array}{l}0.942 \pm 0.012 \\
(130 \text { boutons })\end{array}$ & \\
\hline \multicolumn{5}{|c|}{$\begin{array}{l}\text { Abbreviations: SC, Schaffer collateral; } M F \text {, mossy fiber; } W T \text {, wild-type; Unt, untreated. P-values } \\
\text { in green are from normally distributed data; } p \text {-values in blue are from non-normally distributed } \\
\text { data }\end{array}$} \\
\hline
\end{tabular}




\section{Acknowledgments}

First, I would like to thank Dr. Benjamin H. Cooper for taking me on as his PhD student. With great patience, he showed me everything I know about electron microscopy. His guidance and inspiration led me through my years as a graduate student and I his supervision enabled me to carry out the project to meet high standards. Without him, I would not have been able to accomplish as much as I did.

I am thankful that Prof. Dr. Nils Brose gave me an opportunity to pursue my PhD in his lab. I want to thank him for his support and guidance, but also for his empathy. I could not have made it through this journey without his support.

I would also like to thank Dr. Cordelia Imig for the support and guidance that kept me going throughout my PhD. I would like to thank her for introducing me to the lab and culture system and for making my transition into the lab as smooth as possible. Her balanced motivation and reassurance helped me to push through intense times.

I would like to thank my committee members, Prof. Dr. Tobias Moser and Prof. Dr. Silvio Rizzoli for their scientific feedback during committee meetings and for their support during difficult periods.

Thank you to the additional members of my examination committee, Prof. Dr. Andre Fiala, Prof. Dr. Carolin Wichmann, and Prof. Dr. Stefan Jakobs for taking the time to review my thesis and for taking part in the final test of this thesis. I would also like to thank all members of the examination board for their understanding and flexibility when SARS-CoV-2 derailed my initial examination.

I would like to thank my collaborators. Prof. Dr. JeongSeop Rhee thank you for the help, especially with the electrophysiological experiments. I would like to thank Prof. Dr. Silvio Rizzoli for collaborating on the super-resolution imaging of mossy fiber boutons. I would like to thank Dr. Chungku Lee and Dr. Bekir Altas for performing the electrophysiological experiments and for the many conversations we had in the E-phys room. A huge thank you to Manu Schwark for 
performing all of the animal perfusions as well as the technically challenging task of cryosectioning slice cultures. I would like to thank Sinem Sertel for her hard work on the superresolution imaging and analysis she performed. I wish her the best for her PhD as well. Lastly, Kirsten Weyand analyzed 2D images for me and did an incredible job taking on the task with very little time and kept a positive outlook throughout, I am forever grateful for her dedication and help with the analysis.

Big thanks to Prof. Dr. Carolin Wichmann and Dr. Michael Hoppert for their patience and support when I encountered one of many problems at the tomography electron microscope and came to them for help.

There are many people that I want to thank for helping to edit my thesis: first, Dr. Erinn Gideons who not only helped edit, but did so as a new mom. I do not know where her strength comes from, but she is an inspiration to me. I am so thankful for her help and all moral support she provided along the way. Also, thank you to baby Max for helping Erinn edit my thesis. To Dr. Carolina Thomas for helping to edit my thesis and for guiding me through the bureaucratic paperwork before submission. To Brandi Neifert for the last-minute edits for clarity and readability and for the late-night pep talks. To Kirsten Levandowski for helping edit the thesis from far away. Lastly, to Dr. Andreas Maus for helping to edit my thesis as well as many other writings over the years.

I wish to thank Dr. James Daniel for endless discussion in the office about electrophysiology and about life in general. I also thank Francisco Murcia-Lopez for the discussions, both scientific and non-scientific.

Thank you to the "running group" for the physical activity but mostly for the moral support, storytelling, and small distractions throughout this journey.

I would like to thank Manu for being a truly amazing friend and colleague. She helped lift the dark cloud that frequently hung over my head.

I wish to thank Sabine for the technical support. 
I would also like to thank Noa, Marilynn, and Theo for words of encouragement throughout my time in lab.

I want to say a huge thank you to the PhD students in the Brose department both past and present for making the small community we have in the lab feel like a family. Special thanks to my fellow EM students: Inés, Kirsten, and Valentin for social interactions as well as our small student journal club.

Thank you to Wiebke, Torben, and Boguslawa for friendly advice in the EM core facility.

I would also like to thank all of the PhD Students in the Institute for the regular meetings and internal network provided for all students. The kindness, support, and scientific discussion I've had the pleasure to share and receive was paramount to my success during my PhD.

Thank you to Maria Eichel who provided a lot of support both personal and professional throughout my time at the Institute.

Last but not least, to my family: David, Judy, Channing, Andreas, Elfriede, Walter, Sylvia, Jens, Johanna, Felix, and Jonathan I want to thank you all for the support from far and near during this time of extreme stress and emotion. To my parents for enforcing weekly calls to check in. They helped ground me during times of stress. To my brother, for his wit and encouragement. He was always able to put a smile on my face, even on the darkest days. To my in-laws, thank you for always encouraging me, for accepting me as your family. I am grateful to have a family when I am so far away from home. Finally, to my husband, Andreas. He helped me see the forest for the trees. In times of extremely hard days, he took care that my mental health was also a priority by taking me on micro adventures out into nature. I am so thankful to have him throughout this journey. I cannot wait to experience life outside of the PhD with him.

Special thanks to SARS-CoV-2 for providing one last challenge before submission of my thesis was completed! 


\section{List of Publications}

Imig, C., López-Murcia, F.J, Maus, L., García-Plaza, I.H., Mortensen, L.S., Schwark, M., Schwarze, V., Angibaud, J., Nägerl, U.V., Taschenberger, H., Brose, N., Cooper, B.H. (2020). Ultrastructural Imaging of Activity-Dependent Synaptic Membrane-Trafficking Events in Cultured Brain Slices. Neuron 108, 843-860. dio: 10.1016/j.neuron.2020.09.004.

Maus, L., Lee, C., Altas, B., Sertel, S.M., Weyand, K., Rizzoli, S.O., Rhee, J., Brose, N., Imig, C., and Cooper, B.H. (2020). Ultrastructural Correlates of Presynaptic Functional Heterogeneity in Hippocampal Synapses. Cell Rep. 30, 3632-3643.e8. dio: 10.1016/j.celrep.2020.02.083.

Liu C, Bickford LS, Held RG, Nyitrai H, Südhof TC, Kaeser PS. (2014). The active zone protein family ELKS supports $\mathrm{Ca}^{2+}$ influx at nerve terminals of inhibitory hippocampal neurons. J Neurosci. 34 (37), 12289-303. doi: 10.1523/JNEUROSCI.0999-14.2014.

Tolley M*, Bickford L*, Clare K*, Johann TW. (2012). Investigations of amino acids in the ATP binding site of 5,10-methenyltetrahydrofolate synthetase. Protein J. 6, 519-28. doi:

10.1007/s10930-012- 9428-3.

*co-first authors 